\title{
APPLICATION OF MICROFLUIDIC DEVICES \\ FOR THE STUDY OF BACTERIAL CHEMOTAXIS \\ TO NAPL COMPONENTS IN POROUS MEDIA
}

\author{
A Dissertation \\ Presented to
}

The Faculty of the School of Engineering and Applied Science

University of Virginia

\author{
In Partial Fulfillment \\ of the Requirements for the Degree \\ Doctor of Philosophy (Chemical Engineering) \\ by \\ Xiaopu Wang \\ August 2013
}


APPROVAL SHEET

This dissertation is submitted in partial fulfillment

of the requirements for the degree of

Doctor of Philosophy (Chemical Engineering)

Xiaopu Wang, AUTHOR

This dissertation has been read and approved by the Examining Committee:

Dissertation Advisor

Accepted for the School of Engineering and Applied Science

Dean, School of Engineering and Applied Science

August 2013 


\begin{abstract}
Nonaqueous phase liquid (NAPLs) contaminants are difficult to eliminate from the groundwater system due to their low solubility, low intrinsic reactivity and low release rates from soil or sediments. In situ bioremediation is an economical method to deal with NAPL pollution, but its efficiency is hindered due to the heterogeneous structure in soil. Chemotaxis, the microbial property by which microorganisms sense the concentration gradient of chemicals and migrate towards the preferential regions for their survival and growth, may be a key factor to achieve more efficient bioremediation. A heterogeneous microfluidic device (H- $\mu$ Chip) was designed to mimic features of the natural contaminated groundwater system, with NAPL contaminant trapped in designated locations within the low permeable region. Chemotaxis facilitated the bacteria (P. putida F1) to accumulate adjacent to the contaminated low permeable region as well as in vicinity of the NAPL contaminant sources. Chemotactic bacteria were also more difficult to be washed out from the contaminated region due to their interaction with attractant gradient. Bacteria in the device were also subjected to different flow rates within typical range of groundwater flow rates. A higher flow rate reduced the apparent effect of chemotaxis.
\end{abstract}

Another microfluidic device was fabricated that created a convection free channel (CF$\mu$ Chip), in order to measure the two essential chemotaxis parameters: chemotactic sensitivity coefficient $\chi_{0}$ and chemotactic receptor constant $K_{c}$. These two parameters are the key to quantifying the impact of chemotaxis; however, their values for P. putida $\mathrm{F} 1$ and toluene were not previously measured. This device provided a way to enhance the 
accuracy of the measurement to a great extent compared to conventional methods. By performing the experiment at two different values of the attractant concentration, the two chemotaxis parameters could be determined independently. Chemotactic bacteria exhibited a larger accumulation when the attractant concentration was closer to the correct value of $K_{c}$; therefore, $K_{c}$ could be determined first, and then $\chi_{0}$ could be easily derived after fitting the chemotactic patterns with the correct $K_{c}$ in the mathematical models. Both Pseudomonas putida and Escherichia coli strains were used, and the predicted chemotaxis parameters were consistent with the published results and the values derived from related work.

The numerical solution of mathematical models using COMSOL algorithms yielded outcomes that were consistent with the experimental results, and statistical analysis also supported the experimental comparisons. There is no direct observation on biodegradation, but because toluene is degradable by $P$. putida F1, the experimental observations of biased accumulation of chemotactic bacteria around the NAPL sources within the low permeable region is expected to lead to an increase of contaminant consumption, which can improve the efficiency of bioremediation. 


\section{ACKNOWLEDGEMENTS}

Dr. Roseanne M. Ford, for all your patience, encouragement and support. I gradually understand more about the definition of "research" under your guidance, and I cannot achieve this work without your insight and suggestion.

Dr. Erik Fernandez, Dr. Inchan Kwon, Dr. Ammasi Periasamy and Dr. Javier Atencia for being my committee members. Your suggestions are very enlightening and important for my Ph.D. and future work.

Dr. Javier Atencia, for providing your microfluidic device to me for my Ph.D. research. Dr. Ammasi Periasamy, Dr. Yuansheng Sun and Dr. Vinod Jyothikumar, for the help on the microscope system and fluorescence labeling. Dr. James Landers and Mr. Kerui Xu, for the help on the microfabrication process.

Mom and Dad, for your love. Your love is always the strongest support to me, and I hope you will always be happy because of your only son. I love you.

All my dear friends, for so many memorable moments in my UVa life.

Kaiyi my wife, for stepping into my life. I thought the most beautiful moment in my life was the moment when I met you, but now I realize that, my life becomes more and more wonderful day after day, because of you. I love you.

The National Science Foundation, for the financial support through this project. 


\section{TABLE OF CONTENTS}

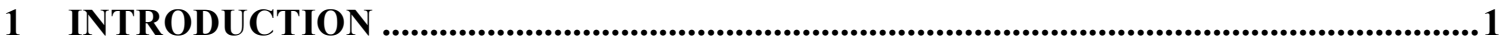

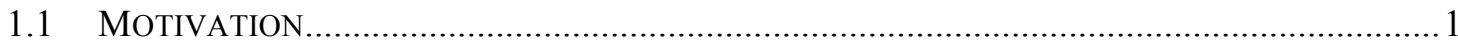

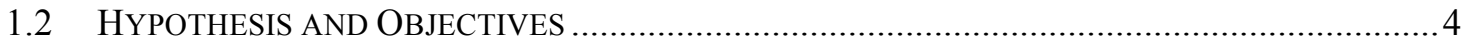

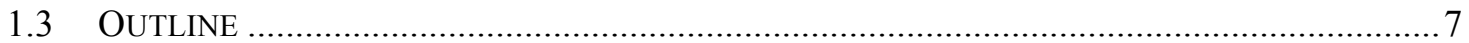

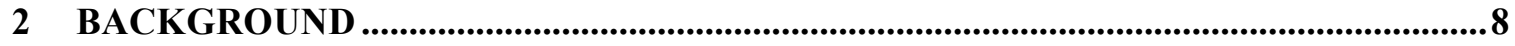

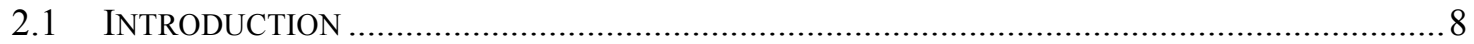

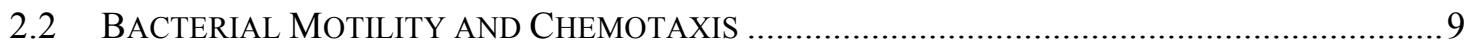

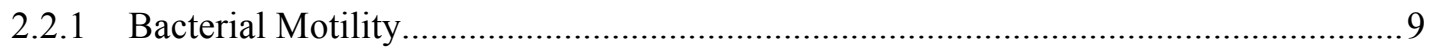

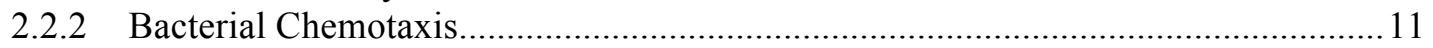

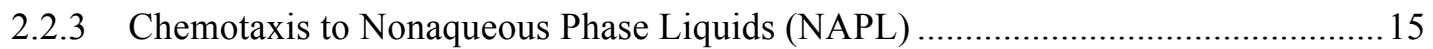

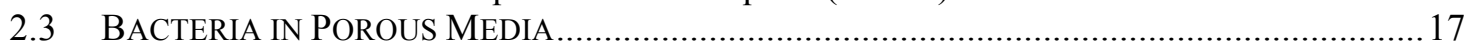

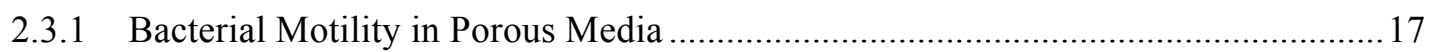

2.3.2 Bacterial Chemotaxis to NAPL in Porous Media....................................................... 18

2.4 APPLICATION OF MICROFLUIDICS IN THIS STUDY AND RELATED RESEARCH.................21

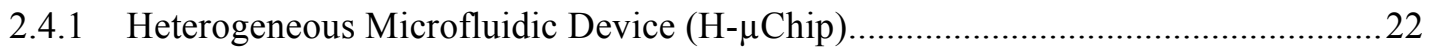

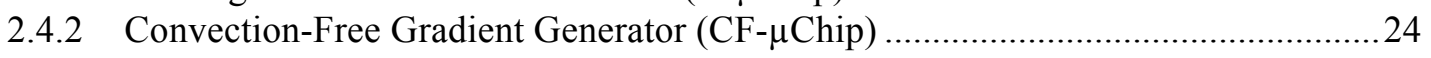

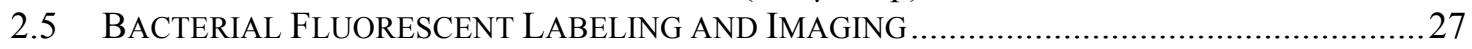

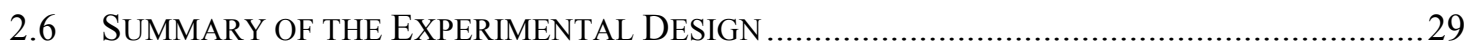

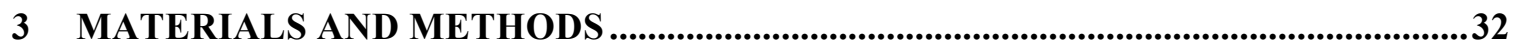

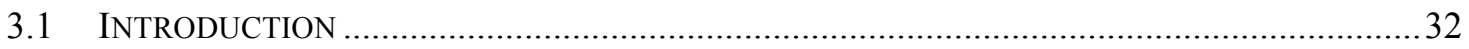

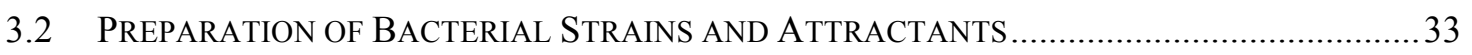

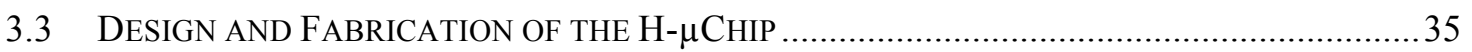

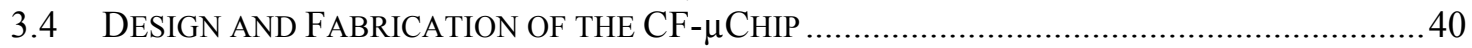

3.5 THE CORRELATION BETWEEN BACTERIAL DENSITY AND FLUORESCENCE INTENSITY ..44

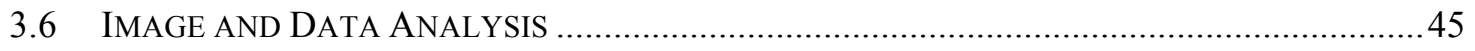

3.7 Mathematical MOdELING, COMPUTATIONAL Simulation AND STATISTICAL

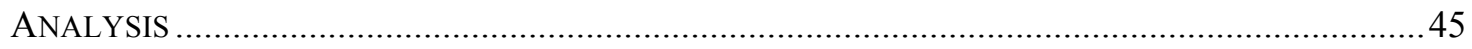

4 СЕ-

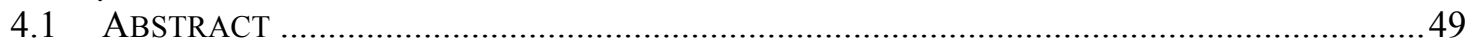

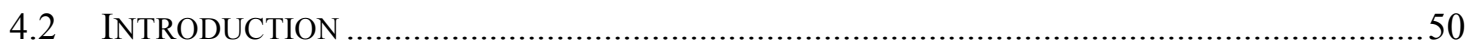

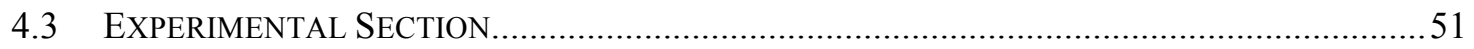

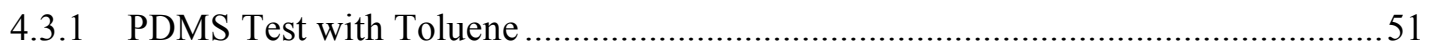

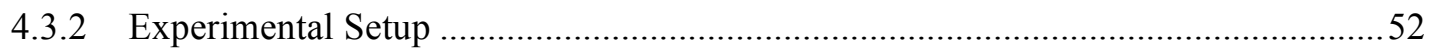

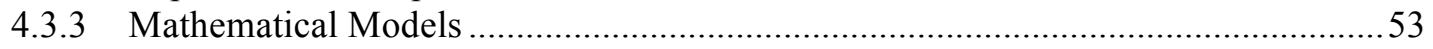

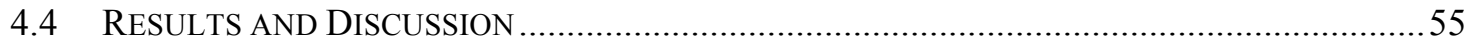

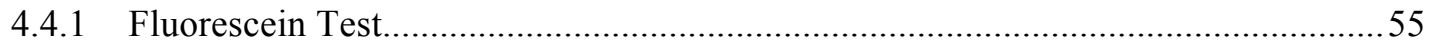

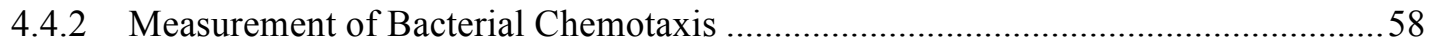

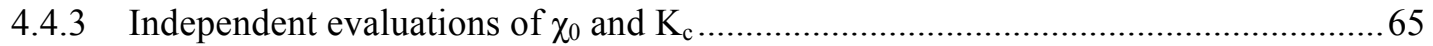

4.4.4 Comparison with previous $\mathrm{K}_{\mathrm{c}}$ measurement .......................................................... 71

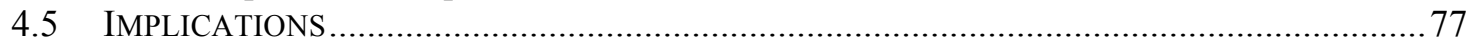

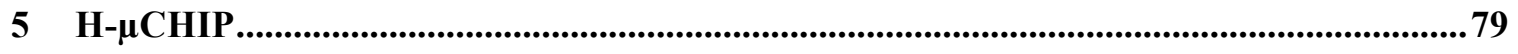

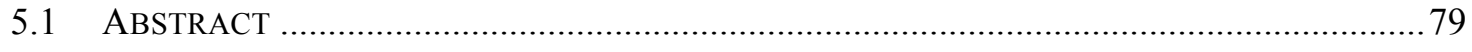

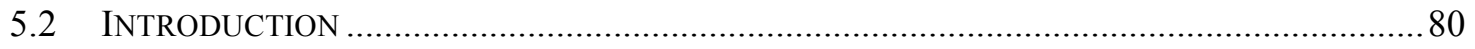




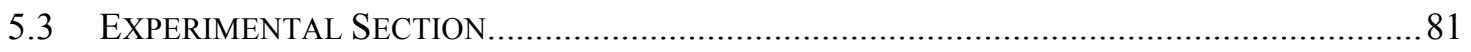

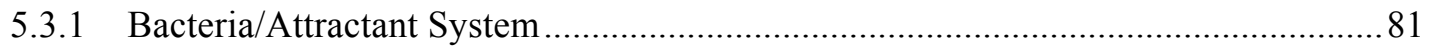

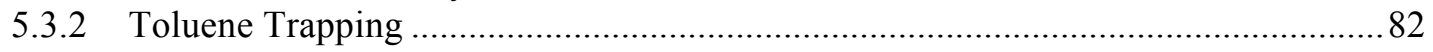

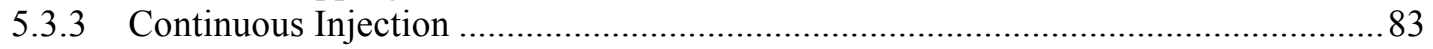

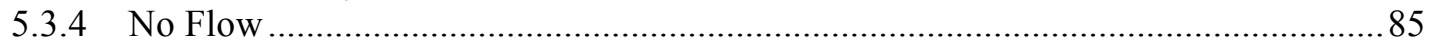

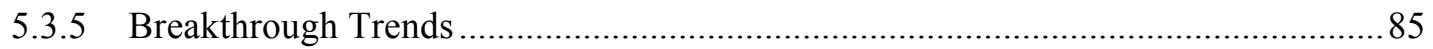

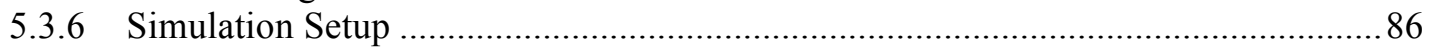

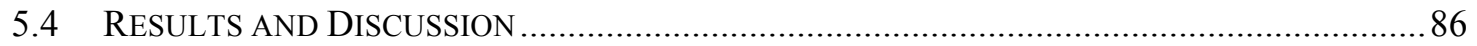

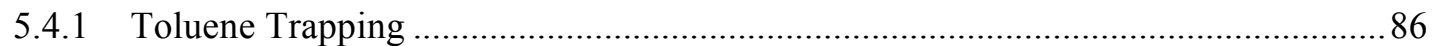

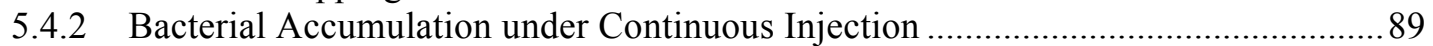

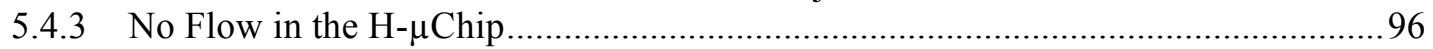

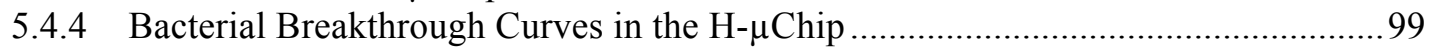

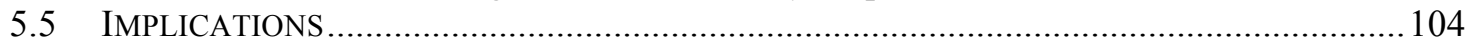

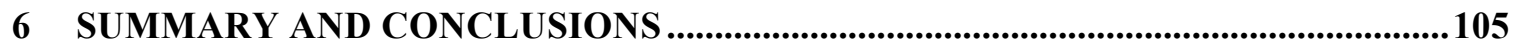

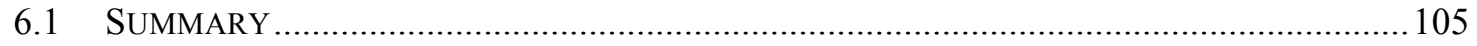

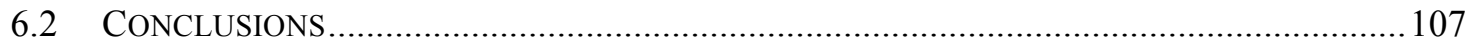

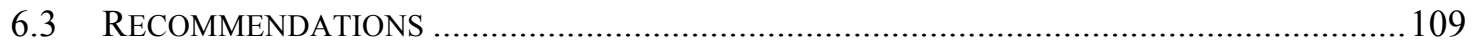

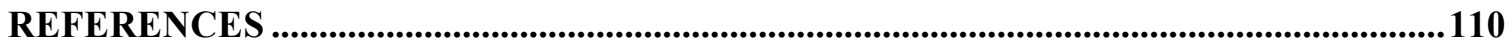

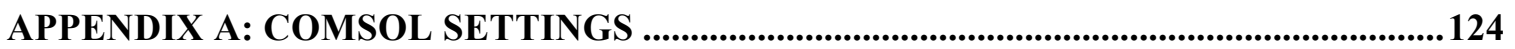

APPENDIX B: TWO-WAY ANOVA ANALYSIS IN CF- $\mu$ CHIP ........................................128

APPENDIX C: TWO-WAY ANOVA ANALYSIS IN H-

APPENDIX D: CORRELATION BETWEEN OPTICAL DENSITY AND LIGHT

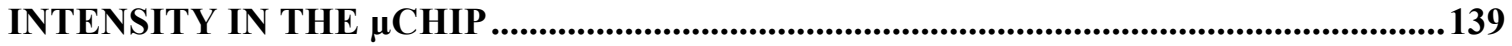

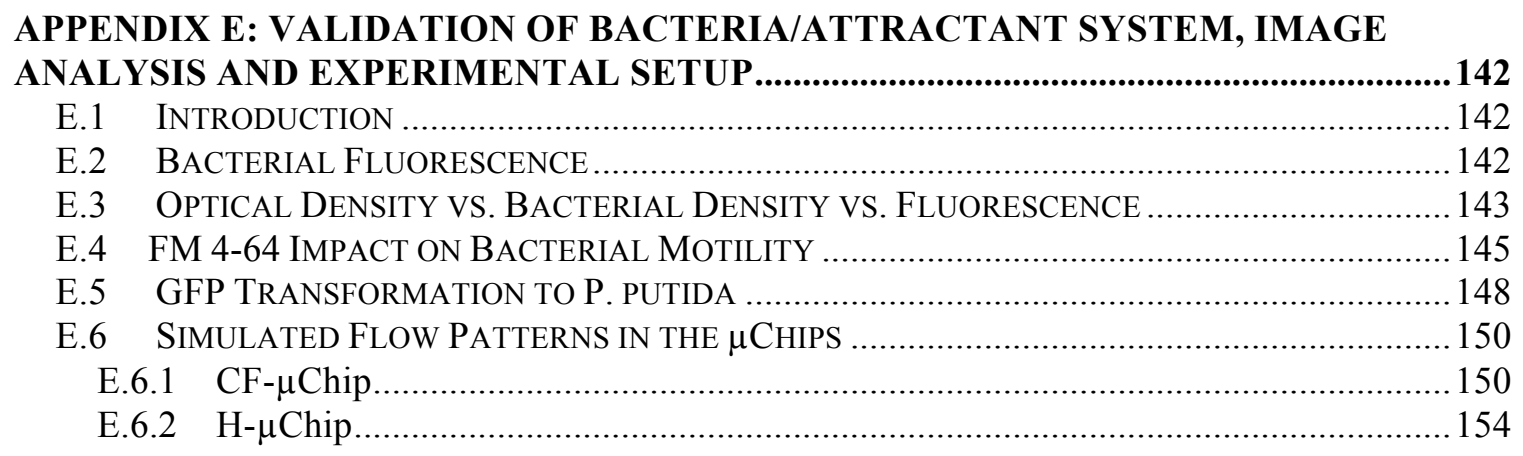




\section{LIST OF FIGURES}

Figure 1.1 Schematic illustration of NAPL contamination in soil (Courtesy from Soga et al., 2004).

Figure 2.1 Peritrichous flagellar bacterium (a) with uncoordinated movement of the flagella, and (b) in the form of a coordinated flagellar bundle. (c) Schematic of individual bacterial movement. Motility $\left(\mu_{0}\right)$ is quantified by bacterial swimming speed $(v)$, run time $\left(\tau_{r}\right)$ and turn angle $(\theta)$.

Figure 2.2 Bacterial random motility and chemotaxis. (a) In an isotropic solution, individual bacterium swims randomly. The straight lines indicate the runs, and the turn points between the lines indicate tumbles. (b) This random motion results in the even bacterial distribution on the macroscopic scale. In the bottom two figures, attractant concentration increases as the area color becomes darker. (c) Chemotaxis increases the run time toward higher attractant concentration gradient, and results in a biased motion toward the area with more attractant. (d) The chemotaxis band forms at higher attractant concentration area from a macroscopic view. Modified from Lanning's $\mathrm{PhD}$ dissertation.

Figure 2.3 The E. coli chemotaxis system. Ligands of stimulus molecules bind to the corresponding receptors on the cell membrane. The bindings result in a series of phosphorylation steps and alter the performance of flagellar motor system, and thus change the run length and tumble frequency on the cellular scale according to the change in the surrounding attractant or repellent concentration (Grebe and Stock, 1998).

Figure 2.4 Schematic of swimming trajectories of $E$. coli in (a) bulk aqueous solution and (b) porous medium. AB represents the same linear displacement of bacterial movement.

Figure 2.5 (a) Schematic representation of bacterial migration in a heterogeneous aquifer (adapted from Kusy, 2005). (b) Same schematic representation in a natural heterogeneous aquifer (modified from LeBlanc et al., 1991). The blue arrows show the preferential pathway for bacteria (green) in the high permeable soil layer, and the area of yellow shaded region indicates the chemical distribution in the low permeable layer.

Figure 2.6 Design of the original porous microfluidic device. (a) The overall diagram illustrates the wide horizontal channel as the macropore with high permeability and the network of micropores as the region with low permeability. (b) A more detailed diagram of the low permeable region shows the dimensions of the channels and ganglia. The depth of the straight pores is approximately $100 \mu \mathrm{m}$, and that of the ganglia is approximately $200 \mu \mathrm{m}$. (c) The detailed diagram shows the patterns etched away from the top and bottom glass pieces in the low permeable region. (d) The toluene droplets have been dyed red and are trapped within the circular ganglia of the micromodel network (adapted from Lanning, 2004).

Figure 2.7 Schematic of green fluorescence protein expression through arabinose catabolism. 
Figure 3.1 (a) Full view of the heterogeneous microfluidic device (H- $\mu$ Chip); the two rounded ends of the horizontal channel are bacterial inlets/outlets, and the third one at the bottom of the device is the NAPL inlet. (b) Zoomed-in view of a part of the fine-grained pore network. Each of the straight channels has a width of $20 \mu \mathrm{m}$. (c) $3 \mathrm{D}$ expanded view of the fine-grained network shown in (b).

Figure 3.2 The basic steps in the procedure of glass etching. The various layers include (a) the mask, (b) the positive photoresist layer, (c) the chromium layer, and (d) the Borofloat glass. The etching process consists of six steps: (I) UV light exposure, (II) development, (III) chromium removal, (IV) acid etch, (V) photoresist removal by stripper, and (VI) final chromium removal.

Figure 3.3 A complete H- $\mu$ Chip with the PDMS blocks at the inlets and outlets. The size of the device is comparable to a US quarter. The red color of the PDMS blocks is resulted from the hydrophobic dye Red Oil O in the toluene after the toluene solution contacts the PDMS polymer.

Figure 3.4 Diagram of convection-free gradient generator (Courtesy of Dr. Javier Atencia). (A) Vias at the same cross section of the horizontal channel (top channel) connect to (B) the two ends of a buried channel in the bottom layer. (C) Two aqueous fluids with different chemical compositions flow parallel to each other in the horizontal channel at same velocity, generating identical pressure at the two symmetric vias, resulting in a diffusion-only linear concentration profile in the buried channel. (D) The vias are away from the diffusive interface between the two fluid streams, and thus the concentration gradient in the buried channel is not affected by the flow oscillation and diffusive region in the horizontal channel. (E) At a desired flow rate, four independent buried channels (a, b, c, d) can achieve the same full concentration gradient of the chemical, and provide replicate conditions for bacteria in the same device. Adapted from Atencia et al., 2012.

Figure 4.1 Illustration of the injection of both the fluorescein solution (light green color) and the $10 \%$ Random Motility Buffer at the same flow rate.

Figure 4.2 Time-lapse distribution of fluorescein solution in the cross channel at times of

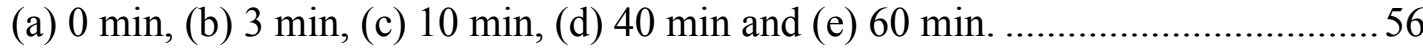

Figure 4.3 Normalized fluorescence of fluorescein within the cross channel at 3, 10 and $60 \mathrm{~min}$. The black curves are the calculated distributions according to the error function solution from Eqn 3.5 using a published value for fluorescein diffusivity of $0.49 \times 10^{-9} \mathrm{~m}^{2} / \mathrm{s}$ (Rani et al., 2005). The coefficient of determination $\mathrm{R}^{2}$ is $0.99 \ldots . .57$

Figure 4.4 Bacterial distribution $(E$. coli $\mathrm{HCB} 1)$ within a cross channel at different times. The gray level intensity in the image corresponds to the bacterial density...... .59

Figure 4.5 The images of $P$. putida distribution in a cross channel at steady state. The gray level intensity represents the bacterial density. The distribution of P. putida F1 is observed in presence of (a) $2 \mathrm{mM}$ toluene as the attractant at the opposite side, and (b) $0.2 \mathrm{mM}$ toluene, and (c) the distribution of P. putida F1 CheA is also recorded in presence of $2 \mathrm{mM}$ toluene.

Figure 4.6 $P$. putida distribution in the cross channel of CF- $\mu$ Chip at steady state. Attractant diffuses from left to right in the figure, and its concentration is $2 \mathrm{mM}$ for the blue diamond data and $0.2 \mathrm{mM}$ for the orange triangles. Experimental results of bacterial density are plotted as dots (nonchemotaxis in red), and the corresponding lines are the fitting results. 
Figure 4.7 The images of $E$. coli distribution in a cross channel at steady state. The gray level intensity represents the bacterial density. The distribution of E. coli HCB1 is observed in presence of (a) $3 \mathrm{mM} \alpha$-methylaspartate as the attractant at the opposite side, and (b) $0.3 \mathrm{mM} \alpha$-methylaspartate, and (c) the distribution of nonchemotactic $E$. coli $\mathrm{HCB} 437$ is also recorded in presence of $3 \mathrm{mM} \alpha$-methylaspartate.

Figure 4.8 E. coli distribution in the diffusion-only channel at steady state. Attractant diffuses from left to right in the figure, and its concentration at the left-hand side is $0.3 \mathrm{mM}$ for the blue diamond data and $3 \mathrm{mM}$ for the orange triangles. Experimental results of bacterial density are plotted as symbols (nonchemotaxis in red), and the corresponding lines are the fitting results.

Figure 4.9 Similar simulation curves resulted from the combinations of different $\chi_{0}$ and $K_{c}$ values.

Figure 4.10 Simulated results of the impact of changing $\chi_{0}$ and $K_{c}$ on bacterial distribution in presence of $2 \mathrm{mM}$ of chemoattractant. The default value for $\chi_{0}$ was set as $1 \times 10^{-8} \mathrm{~m}^{2} / \mathrm{s}$ and for $K_{c}$ as $0.1 \mathrm{mM}$, and (a) shows the impact of $\chi_{0}$ with a fixed $K_{c}$ at the default value, and (b) shows the impact of $K_{c}$ with a fixed $\chi_{0}$ at the default value.

Figure 4.11 Simulated results about the impact of changing $\chi_{0}$ and $K_{c}$ on bacterial distribution in presence of $0.2 \mathrm{mM}$ of chemoattractant. The default values for $\chi_{0}$ is set as $1 \times 10^{-8} \mathrm{~m}^{2} / \mathrm{s}$ and $K_{c}$ as $0.1 \mathrm{mM}$, and (a) shows the impact of $\chi_{0}$ with a fixed $K_{c}$ at the default value, and (b) shows the impact of $K_{c}$ with a fixed $\chi_{0}$ at the default value.

Figure 4.12 Agarose plug assay diagram with (a) top view and (b) side view (Courtesy of Brashear).

Figure 4.13 Chemotactic response of $P$. putida $\mathrm{F} 1$ to sodium benzoate at different concentrations in agarose plug assay (Brashear, 2009). .73

Figure 4.14 (a) Simulation of chemotactic bacterial distribution with different attractant concentration at the bacterial end. The solid curve is generated from the zero attractant background, and the dashed curve is from the background with the diluted attractant concentration at the bacterial end. (b) The differences between the two curves in (a) along the channel direction.

Figure 4.15 Comparison between the benzoate related data (open triangle) from plug assay and the toluene related data (solid square) from CF- $\mu$ Chip. The black line connects the fitting data for bacterial distribution to toluene.

Figure 5.1 Schematic illustration of the bacterial injection in $\mathrm{H}-\mu \mathrm{Chip}$. The red color represents the toluene ganglia, and the arrows indicate the inlet, the outlet and the flow direction for bacteria.

Figure 5.2 The predicted attractant concentrations for both the original and the new H$\mu$ Chip in the horizontal channel were compared along the red lines in the image. .. 85

Figure 5.3 Toluene dyed with Oil Red $\mathrm{O}$ was retained as ganglia in the microfluidic device. A light blue colored aqueous solution was also injected to illustrate the channel patterns.

Figure 5.4 Cartoon illustration of the toluene entrapment in $\mathrm{H}-\mu \mathrm{Chip} . \mathrm{F}_{\mathrm{s}}$ stands for the force vector induced from surface tension on a toluene ganglion, and $\mathrm{F}_{\mathrm{w}}$ represents the resistance force from the glass wall. 
Figure 5.5 The simulated attractant distribution along the centerline in the horizontal channel for both the original (black) and the new (gray) $\mathrm{H}-\mu \mathrm{Chip}$ at $0.5 \mathrm{~m} / \mathrm{d}$........ 91

Figure 5.6 The simulated attractant distribution along the transverse direction in the horizontal channel for both the original (black curves) and the new (gray curves) $\mathrm{H}$ $\mu$ Chip at $0.5 \mathrm{~m} / \mathrm{d}$. Number 1 to 3 increases from left to right in Figure 5.2, as the flow is injected from the inlet on left.

Figure 5.7 Distribution of chemotactic (light gray square) and nonchemotactic (dark gray circle) bacteria at different junctions with a fluid velocity of $0.5 \mathrm{~m} / \mathrm{d}$. The connecting lines indicate the fitted data for accumulation of chemotactic bacteria at the junctions, and the fitted results for nonchemotactic bacteria respectively.

Figure 5.8 Distribution of chemotactic bacteria at different junctions under different convective flow rates, which are $0.5 \mathrm{~m} / \mathrm{d}$ (gray square) and $5 \mathrm{~m} / \mathrm{d}$ (black triangle) respectively. The connecting lines indicate the fitted data for bacterial accumulation at the junctions with flow rate of $0.5 \mathrm{~m} / \mathrm{d}$, and the fitted results for that at $5 \mathrm{~m} / \mathrm{d}$ respectively.

Figure 5.9 (a) Cartoon illustration and (b) image of chemotactic bacteria near a NAPL source. Each individual bright spot in (b) is a fluorescent bacterium dyed by FM 464 , and (c) is a composite average from over 30 images of (b) at different locations around the toluene-water interface of a single ganglion.

Figure 5.10 The distribution of chemotactic and nonchemotactic bacteria near NAPL sources.

Figure 5.11 The breakthrough curves of chemotactic and nonchemotactic bacteria at different flow rates. "chtx" and "nonch" in the legend stand for chemotactic and nonchemotactic bacteria respectively. The lines are the fitting results corresponding to different experimental datasets (dataset number $n=3$ ). The fittings were run at a time interval of $20 \mathrm{~min}$. 


\section{LIST OF TABLES}

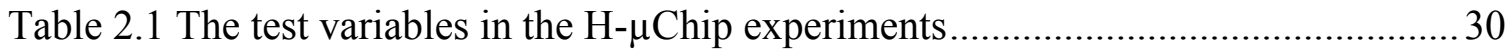

Table 2.2 Comparisons among the $\mathrm{H}-\mu \mathrm{Chip}$ study and previous representative works ... 30

Table 2.3 The test variables in the CF- $\mu$ Chip experiments............................................... 31

Table 4.1 Calculated time to reach steady state for all bacterial strains and chemicals used

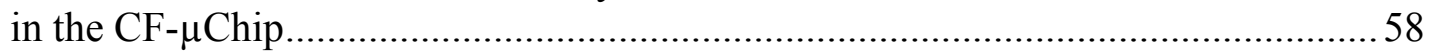

Table $4.2 \chi_{0}$ and $K_{c}$ values used for the different simulation curves in Figure 4.9.........66

Table 6.1 Main parameters for the computational simulation of bacterial chemotaxis .. 109 


\section{LIST OF SYMBOLS}

Symbol

Arabic

$a$

$b$

$c$

$d$

D

I

$K_{c}$

$\mathbf{N}$

$p$

$t$

$u_{\text {avg }}$

$\mathbf{u}$

$v_{b}$

$v_{C h}$

$x$
Definition [Unit]

attractant concentration $\left[\mathrm{moles} / \mathrm{L}^{3}\right]$

bacterial density $\left[\right.$ moles $\left./ \mathrm{L}^{3}\right]$

species concentration $\left[\right.$ moles $\left./ \mathrm{L}^{3}\right]$

depth of channel [L]

diffusion coefficient $\left[\mathrm{L}^{2} / \mathrm{T}\right]$

light intensity in 8-bit images

chemotaxis receptor constant $\left[\right.$ moles $\left./ \mathrm{L}^{3}\right]$

flux $\left[1 / \mathrm{L}^{2} \mathrm{~T}\right]$

pressure $[\mathrm{P}]$

time [T]

average velocity $[\mathrm{L} / \mathrm{T}]$

flow velocity vector $[\mathrm{L} / \mathrm{T}]$

individual bacterial swimming speed [L/T]

chemotactic velocity $[\mathrm{L} / \mathrm{T}]$

distance in $\mathrm{X}$-direction [L] 
Greek

$\chi_{0}$

$\lambda$

$\mu_{0}$

$\mu$

$\tau_{r}$

$\theta$

$\rho$

$\varepsilon_{p}$

$\kappa_{b r}$ distance in y-direction [L]

distance in z-direction [L] chemotactic sensitivity coefficient $\left[\mathrm{L}^{2} / \mathrm{T}\right]$

run length between tumbles [L]

bacterial random motility coefficient $\left[\mathrm{L}^{2} / \mathrm{T}\right]$

dynamic viscosity [P T]

bacterial run time $[\mathrm{T}]$

turn angle between two successive bacterial runs

fluid density $\left[\mathrm{M} / \mathrm{L}^{3}\right]$

porosity

permeability of the porous medium $\left[\mathrm{L}^{2}\right]$ 


\section{INTRODUCTION}

\section{Chapter 1}

\section{INTRODUCTION}

\subsection{Motivation}

Nonaqueous phase liquids (NAPL) are the most common industrial contaminants in groundwater pollution (Knutson et al., 2001). NAPL contaminants, including BTEX (benzene, toluene, ethylbenzene, and xylenes), trichloroethylene (TCE), petroleum and chlorinated hydrocarbons (Newell et al., EPA), are difficult to eliminate from natural aquifers due to their low solubility, low volatility, low intrinsic reactivity and low release rates from soil or sediments (Pignatello and Xing, 1996; Ang et al., 2005). As a 
consequence, they have become a major threat to the groundwater sources for our daily usage.

Many remediation techniques and strategies are developed to eliminate this longterm threat. These methods include pump-and-treat, injection of catalytic metal particles, excavation, thermal desorption, air sparging and multi-phase extraction (Liu et al., 2005; Boopathy, 2000), and are generally high-cost and labor-intensive. The U. S. Environmental Protection Agency reported that over half billion kilowatt-hours (kWh) of energy was consumed for the most frequently applied remediation treatments at the National Priority List (NPL) sites every year (EPA, 2008), and the energy cost was predicted double in 2030. The induced economic and environmental burden behind the energy cost was something that could not be ignored. Therefore, a more economical alternative choice was needed.

In-situ bioremediation provides such an effective and economical solution to remove the organic pollutants from groundwater (Hart, 1996; Jorgensen, 2007; Scow et al., 2005). Generally speaking, indigenous microorganisms or other forms of biomass are injected into the contaminated sites and directly degrade or transform contaminant chemicals into non-toxic byproducts (Grindstaff, 1998); at the same time, microorganisms may grow and increase their population upon the contaminant sources, which can accelerate the biodegradation process. In-situ bioremediation quickly gained popularity among various treatment options, because it is cheaper and more effective than the conventional approaches.

However, in-situ bioremediation has its own limitations. Because of the heterogeneity of the natural groundwater systems, the NAPL contaminant sources are 
easily trapped in regions where the conductivity is fairly low, either coating on the surface of soil grains or hiding in the narrow pore spaces (Figure 1.1). Thus, these NAPL residuals are hard to be carried away by the convective flow in adjacent zones with high hydraulic conductivity (Alexander, 1994; Harms and Wick, 2006; Gierczak et al., 2006). One prominent example is the oil spill from the tanker Exxon Valdez in 1989. Although soluble contaminants were removed from the high-permeability upper layer of the beach by conventional remediation, residual oil persists in the low-permeability lower layer, and continues to leach slowly into the upper layer ( $\mathrm{Li}$ and Boufadel, 2010). Therefore, a more efficient method is needed to remediate the low permeable regions, in order to ensure complete removal of oil.

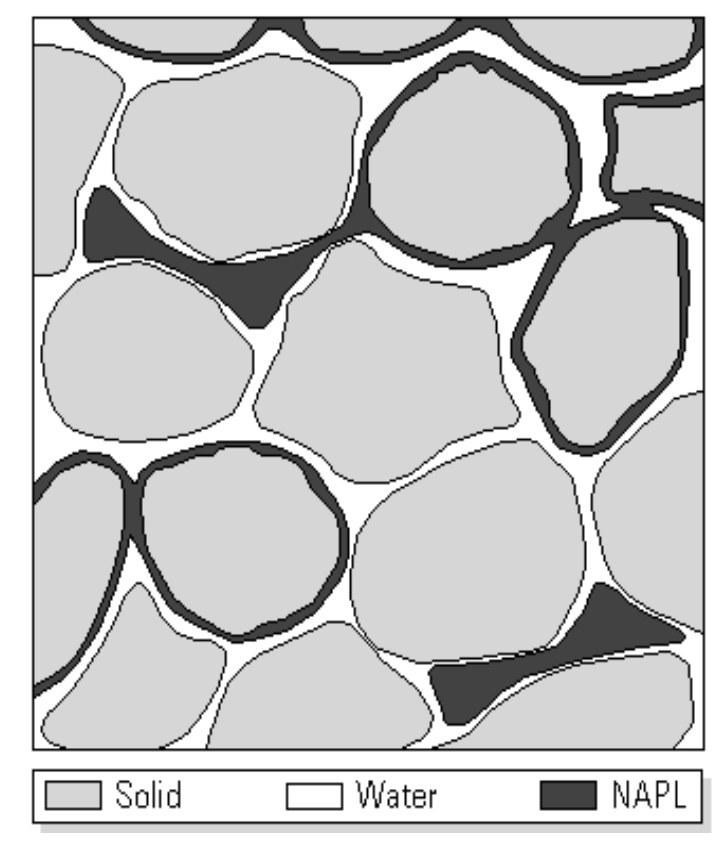

Figure 1.1 Schematic illustration of NAPL contamination in soil (Courtesy from Soga et al., 2004).

Chemotaxis, the microbial property by which microorganisms sense the concentration gradient of chemicals and migrate towards the preferential regions for their 
survival and growth, may be a key factor to achieve more efficient bioremediation (Pandey and Jain, 2002; Ford and Harvey, 2007). Chemotaxis phenomena were associated with the biodegradation of many environmental chemicals, including NAPL and various aromatic compounds; because of the ability to locate and degrade the pollutants, such bacteria have a selective advantage to survive and grow in various contaminated sites, and as a consequence, reduce the pollutants in these areas (Harms and Wick, 2006; Pandey and Jain, 2002; Harwood et al., 1984; Parales et al., 2000; Lopez-deVictoria and Lovell, 1993; Grimm and Harwood, 1997; Law and Aitken, 2003; Harwood et al., 1990; Hawkins and Harwood, 2002; Bhushan et al., 2000; Samanta et al., 2000). An indigenous bacterial strain $P$. putida F1 was studied, because it showed strong chemotactic responses to and degraded many chlorinated hydrocarbons, such as toluene, benzene, trichloroethylene etc. (Harwood et al., 1990; Parales et al., 2000), and was widely applied to study bacterial chemotaxis. In this study chemotaxis was expected to facilitate targeting the contaminant sources in the contamination zone, and to exhibit different behavior from nonchemotactic controls. Two key transport parameters of chemotaxis were also experimentally measured to assess the chemotactic ability in both bulk fluid and porous media.

\subsection{Hypothesis and Objectives}

As mentioned above, chemotaxis is a bacterial property that is common to many indigenous microorganisms, and may be important in in-situ biodegradation processes of different chemical pollutants, including NAPLs and various aromatic compounds. Although many studies demonstrate that chemotaxis is quite promising to enhance the biased bacterial migration and effectiveness of bioremediation in porous media, no 
quantitative studies have been reported for chemotaxis in a heterogeneous porous medium with two phases (aqueous and nonaqueous phases). A two-phase heterogeneous porous system is more representative of NAPL contaminated sites, and thus will be more beneficial in understanding the role of chemotaxis in in-situ bioremediation. Therefore, in this dissertation I investigated the effect of chemotaxis on bacterial accumulation in the vicinity of residual NAPL contaminants, which are trapped in the less permeable zone of a porous microfluidic device. As one defining characteristic of contaminated sites is the groundwater flow rate, I also studied the effect of flow rate on accumulation of chemotactic bacteria in the less permeable zone.

Microfluidics facilitates this study in several different aspects. The main advantage is that it allows a predictable pore structure, so both the low and high permeable zones are well defined. This feature avoids the complications in conventional sand-packed systems, such as nonuniform packing and makes the experimental platform more controllable. In addition, its outstanding light transparency allows direct observation of bacterial movement and NAPL distribution inside the device in real time under the microscope. Moreover, the precise microscale fabrication in a microfluidic device allows one to mimic certain features that are critical for representing groundwater environment. The small size of microfluidic devices also reduces the long time needed for conventional studies on biodegradation. Therefore, based on the properties of microfluidics and the mechanism of chemotaxis, my hypothesis was:

\author{
In well-defined heterogeneous porous media, chemotaxis will facilitate bacterial \\ migration preferentially towards NAPL attractant sources trapped in regions with
}




\section{lower hydraulic conductivity, which will lead to accumulation of bacteria at chemically favorable locations. Furthermore, the groundwater flow rate will affect the impact of chemotaxis in the heterogeneous porous system.}

Due to preferential migration toward chemoattractants such as toluene, chemotactic bacteria were expected to accumulate to a greater extent than nonchemotactic controls near the contaminant source in the small-scale pore network. When the convective flow rate, which was chosen in the typical velocity range of groundwater flow, was comparable to or lower than the bacterial average swimming speed, a strong chemotactic response to the chemoattractant was expected. However, if the convective flow rate was above the bacterial average swimming speed, the bacterial accumulation was expected to diminish, to the point of not producing a measurable difference from the experimental controls. Therefore, in order to test my hypothesis, I designed a series of experiments and simulation algorithms to:

(1) investigate the significance of chemotaxis on bacterial transverse migration towards NAPL attractant trapped in the low permeable porous zone,

(2) explore the influence of convective flow on bacterial accumulation in vicinity of the source of organic chemicals in the heterogeneous system,

(3) use a novel microfluidic device to evaluate the characterization parameters of chemotaxis, chemotactic sensitivity coefficient and chemotactic receptor coefficient, for all combinations of bacterial strains and chemoattractants in this study. 


\subsection{Outline}

Chapter 2 focuses on the fundamental background knowledge about the key concepts in this study, points out the pros and cons of previous relevant works, and summarizes the main research goal. Chapter 3 introduces the bacteria/attractant pairs, protocols and mathematical models, to help understand the outcomes in the following chapters. Chapters 4 and 5 are the main contents of this dissertation. Chapter 4 concentrates on the quantification of the key chemotaxis parameters by using a convection-free microfluidic device and corresponding mathematical models. Chapter 5 introduces a novel microfluidic system that successfully traps an organic contaminant in assigned positions, shows the bacterial accumulation in the vicinity of these contaminant sources due to chemotaxis, and reveals the impact of heterogeneity and flow rate on bacterial distribution. Chapter 6 concludes the dissertation, proposes unanswered questions, and recommends guidelines for future research. 


\section{BACKGROUND}

\section{Chapter 2}

\section{BACKGROUND}

\section{$2.1 \quad$ Introduction}

The main purpose of this chapter is to introduce the key concepts in the dissertation, and briefly explain the rationale behind the selection of the materials and the experimental design.

Section 2.2 presents the mechanisms of bacterial motility and chemotaxis in bulk aqueous solution, and emphasizes the chemotaxis ability to a main group of groundwater contaminants, nonaqueous phase liquids (NAPL). Section 2.3 describes how porous media affect bacterial motility and chemotaxis and limit NAPL bioremediation in fieldscale applications, and provides a rationale for why chemotactic bacteria may enhance insitu bioremediation. Section 2.4 briefly reviews the development of microfluidic devices 
related to NAPL and chemotaxis study, and proposes two novel microfluidic devices: heterogeneous microfluidics (H- $\mu$ Chip) for quantification of chemotaxis to NAPL within a heterogeneous porous matrix, and convection-free gradient generator (CF- $\mu$ Chip) for measurement of key chemotaxis parameters. Section 2.6 introduces several fluorescencelabeling methods to improve the quality of image data for bacterial distributions; these methods include green fluorescent protein (GFP) transformation, live membrane staining with FM 4-64 and DAPI staining. Finally, Section 2.7 is a summary of the implications from the works in the previous sections in this chapter to my own experimental design.

\subsection{Bacterial Motility and Chemotaxis}

\subsubsection{Bacterial Motility}

In an isotropic bulk aqueous solution, the individual swimming behavior of motile bacteria can be described as a three-dimensional random walk, which is similar to Brownian motion. However, these two concepts are still different mainly in that the bacterial swimming pattern is caused by flagellar rotation, while Brownian motion is caused by molecular collision. The three-dimensional random walk consists of a series of runs and tumbles. For a peritrichous flagellar bacterium, such as Escherichia coli, a run happens when the flagella rotate in a coordinated counter-clockwise direction to form a flagellar bundle (Figure 2.1b), and the rotation propels the bacterium to move forward. Then, a tumble occurs when one of more of the flagella reverses the direction of rotation, as a result, the flagella bundle unravels temporarily (Figure 2.1a), after which the bacterium begins the next run in a new direction. Thus, an individual bacterial swimming pattern is composed of a series of runs and tumbles, as shown in Figure 2.1c. 
a

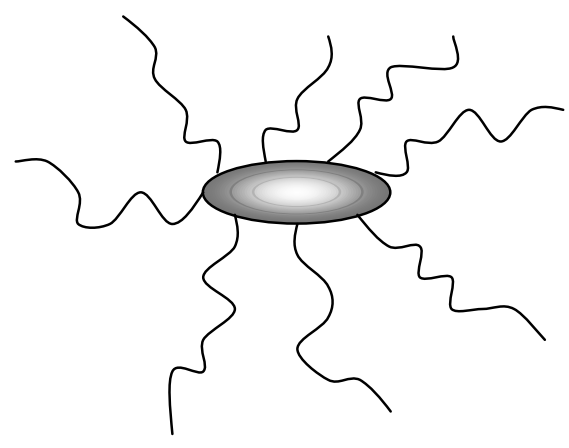

b

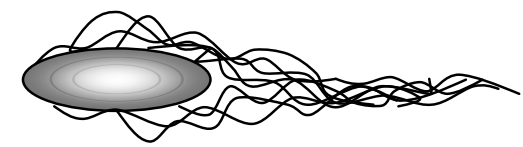

$\mathrm{c}$

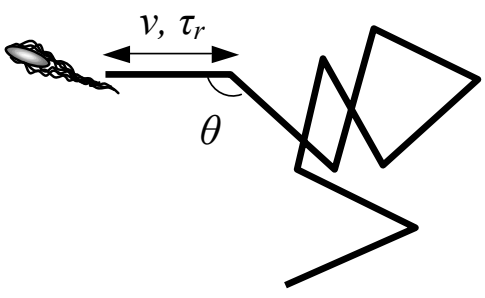

Figure 2.1 Peritrichous flagellar bacterium (a) with uncoordinated movement of the flagella, and (b) in the form of a coordinated flagellar bundle. (c) Schematic of individual bacterial movement. Motility $\left(\mu_{0}\right)$ is quantified by bacterial swimming speed $(v)$, run time $\left(\tau_{r}\right)$ and turn angle $(\theta)$.

Diffusion coefficients are used to quantify molecular diffusion; similarly, the bacterial random motility coefficient $\left(\mu_{0}\right)$ is used to describe the bacterial random motility. The following mathematical expression relates the random motility coefficient and the parameters of run-tumble mechanism (Lovely et al., 1997):

$$
\mu_{0}=\frac{v \lambda}{3} \frac{1}{1-\langle\cos \theta\rangle}
$$

where $v$ is the individual bacterial swimming speed, $\lambda$ is the run length between tumbles. $\lambda$ is the product of $v$ and $\tau_{r}$ (run time), as shown in Figure 2.1c. $\theta$ is the turn angle 
between two successive runs, and averaged for an individual bacterium from a series of changes in swimming directions, and \langle\rangle represents the average quantity.

\subsubsection{Bacterial Chemotaxis}

For certain bacterial strains, bacterial run length $(\lambda)$ and tumble frequency $\left(1 / \tau_{r}\right)$ can be altered by the ambient chemical concentration. When these bacteria swim up the attractant gradient, their run length increases as their tumble frequency decreases, resulting in an overall biased movement towards the locations with higher chemical concentration (Figure 2.2). This swimming behavior refers to chemotaxis. Bacterial chemotaxis is a microbial phenomenon in which bacteria respond to the concentration gradients of specific chemicals within the surrounding environment and biases the threedimensional random walk toward favorable conditions or away from unfavorable conditions. In this dissertation, chemotaxis is referred as the biased bacterial movement toward favorable conditions rather than away from unfavorable conditions, if no additional explanation is provided.

On the molecular scale, bacterial chemotaxis in E. coli is regulated by a signal pathway between two groups of membrane-bound protein complexes: the methylaccepting chemotaxis proteins complexes (MCPs), which are the receptor complexes and mainly located at the pole(s) of the cell body, and the flagellar-motor complexes, which are randomly distributed over the cell membrane (Poole, 1990). Five different protein complexes on the inner cellular membrane have been identified that collect and pass along the signal from external chemical stimuli: $\operatorname{Trg}$ (taxis toward ribose and galactose) and Tar (taxis toward aspartate and away from certain repellents) are the two major ones; Tsr (taxis toward serine and away from certain repellents), Tap (taxis 
toward di- and tri-peptides) and Aer (aerotaxis) are the minor ones (Grebe and Stock, 1998). The first four receptors are MCPs, and Aer is an MCP-like transmembrane protein; Aer is found to be a cellular redox detector, which monitors external oxygen level (Bibikov et al., 1997; Rebbapragada et al., 1997).

As shown in Figure 2.3, when the chemoattractant ligand binds to the MCPs, the receptors become methylated and deliver the signal from the attractant quickly along the chemotaxis pathway through adaptor proteins, CheW and CheA. As a result, a kind of messenger protein on this pathway, CheY, becomes dephosphorylated (CheY). Phosphorylated CheY (CheY-P) can bind to switching proteins at the flagellar motor, and make flagellar rotate clockwise, and thus cause bacterial tumbling. Therefore, an increase in the concentration of attractant decreases the amount of CheY-P, reducing the frequency of bacterial tumbling, causing the bacteria to run longer. Conversely, when bacteria swim downstream of the attractant gradient, the level of CheY phosphorylation goes up, and consequently, bacteria tumble more frequently, presumably to test new directions toward the locations with higher chemical concentration. The methylation of MCPs is a reversible reaction. As the MCPs are demethylated, the $\mathrm{CheY}$ becomes phosphorylated again and the tumble frequency resumes its base level. CheR and CheB, the methylesterases, are instrumental in the adjustment of this reversible process. CheR can be activated by the dephosphorylation of CheA, and then transfers methyl groups to the cytoplasmic signaling domains of the MCPs in the presense of chemoattractant; CheB impacts the process in the opposite manner. These covalent receptor modifications can activate or reset the status of receptors, and thus allow the bacteria to respond to the changes in surrounding chemical concentration. Aer chemotaxis signaling pathway 
involves another mechanism without phosphorylation. Hexoses such as glucose are phosphorylated by enzyme Pts I and transported through PEP system. The dephosphorylated Pts I inhibits the activity of CheA (Macnab and Koshland, 1972).

On the cellular scale, the changes within chemotaxis pathway result in a series of changes of the run times in difference directions in presence of chemical concentration gradient. Generally speaking, the run time increases for a preferable direction, and vice versa. In this way, bacteria swim toward a favorable environment in the process of a chemotaxis response (Figure 2.2).

On the population scale, as depicted in Figure 2.2, bacterial chemotaxis leads to a relatively high bacterial concentration accumulation at certain high chemoattractant concentration region within its gradient, instead of even distribution for nonchemotactic bacteria or no chemical gradient environment. 


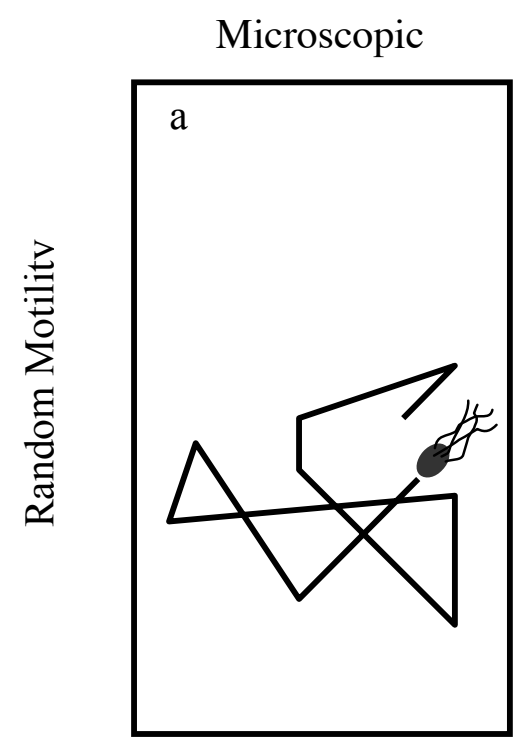

\section{Macroscopic}
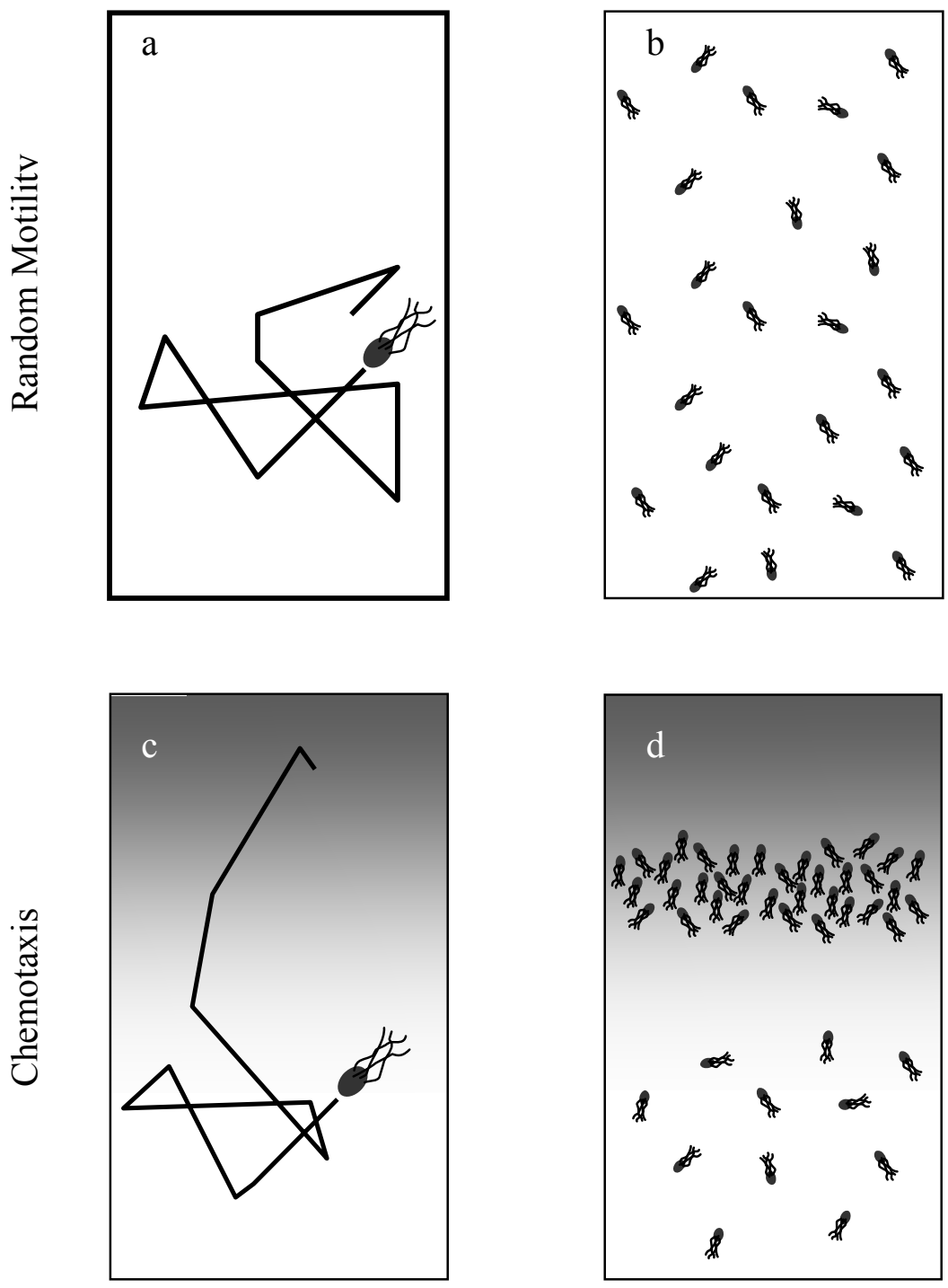

Figure 2.2 Bacterial random motility and chemotaxis. (a) In an isotropic solution, individual bacterium swims randomly. The straight lines indicate the runs, and the turn points between the lines indicate tumbles. (b) This random motion results in the even bacterial distribution on the macroscopic scale. In the bottom two figures, attractant concentration increases as the area color becomes darker. (c) Chemotaxis increases the run time toward higher attractant concentration gradient, and results in a biased motion toward the area with more attractant. (d) The chemotaxis 
band forms at higher attractant concentration area from a macroscopic view. Modified from Lanning's PhD dissertation.

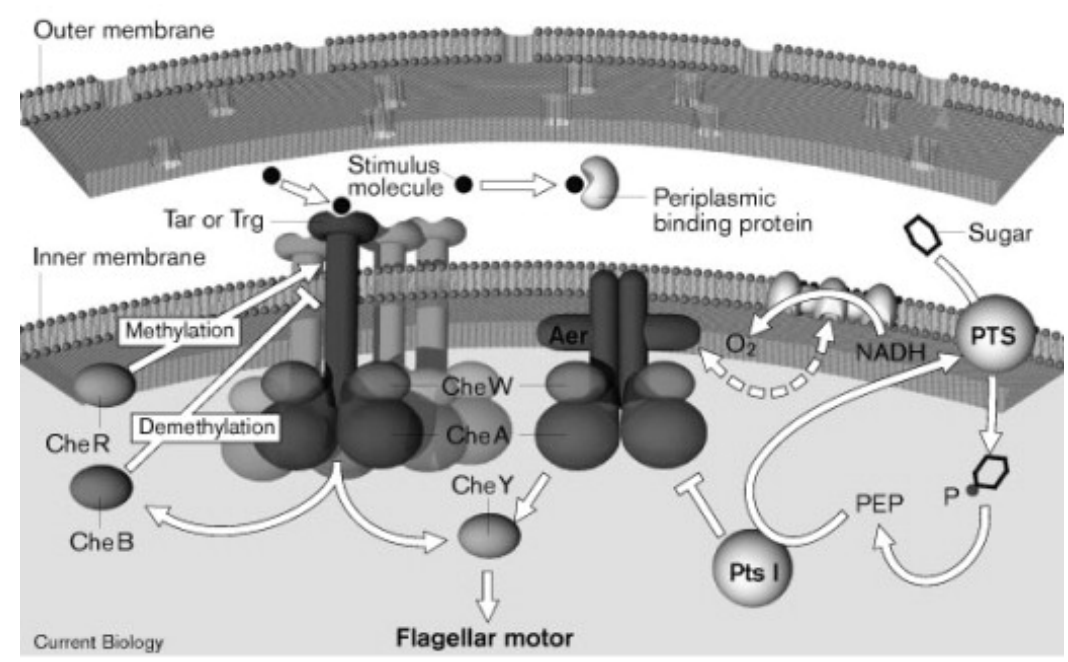

Figure 2.3 The E. coli chemotaxis system. Ligands of stimulus molecules bind to the corresponding receptors on the cell membrane. The bindings result in a series of phosphorylation steps and alter the performance of flagellar motor system, and thus change the run length and tumble frequency on the cellular scale according to the change in the surrounding attractant or repellent concentration (Grebe and Stock, 1998).

\subsubsection{Chemotaxis to Nonaqueous Phase Liquids (NAPL)}

The disposal of organic solvents used in modern industries leads to an increase in chemical pollution of groundwater, and consequentially results in an increasing threat to human health. Organic solvents in the form of the nonaqueous phase liquid (NAPL) are the most widely distributed and most threatening pollutants in groundwater system (Newell et al., 1994). NAPLs exist in nature as a separate and immiscible phase from water or air, and are simply classified into light NAPLs (LNAPLs) and dense NAPLs 
(DNAPLs) based on their density comparison with water. Both LNAPLs and DNAPLs include a wide range of common contaminants. The typical LNAPL components are benzene, toluene, ethylbenzene and xylenes, which are also known as BTEX (Newell, EPA), which can be found in all cruel oil resources. 1,1,2-Trichloroethylene (TCE) and naphthalene are the most common DNAPL contaminants in the subsurface (Kavanaugh, EPA). In this dissertation, the NAPL of interest is toluene. Toluene is a very common organic solvent adopted by various industrial fields, and it becomes one of the main NAPL contaminants in the groundwater system. The NAPL contaminants are fairly toxic to life, for example, long-term exposure to toluene can cause many toxic effects on human body, including psychoorganic syndrome, visual evoked potential (VEP) abnormality, toxic polyneuropathy, cerebellar, cognitive, and pyramidal dysfunctions, optic atrophy, and brain lesions (Miyagi, 1999; Urban, 2003); TCE is reasonably listed as a human carcinogen in the Eleventh Report of Carcinogens from National Institute of Health in 2005.

Several natural microbial species are involved in degradation of many different NAPL contaminants and are reported continuously over the past decades (Farhadian, 2008). The cases include degradation to simple aromatic compounds (Harwood et al., 1984; Parales et al., 2000; Lopez-de-Victoria and Lovell, 1993), polycyclic aromatic hydrocarbons (PAHs; Grimm and Harwood, 1997; Law and Aitken, 2003), chloroaromatic compounds (Harwood et al., 1990; Hawkins and Harwood, 2002) and nitroaromatic compounds (Bhushan et al., 2000; Samanta et al., 2000). Amongst all these degrading microorganisms, many different bacterial strains are proven to exhibit the abilities of both chemotaxis and biodegradation in the presence of NAPL contaminants. 
The slow dissolution of the NAPL droplets into the surrounding aqueous environment forms a concentration gradient, then the chemotactic bacteria change their threedimensional random walk correspondingly, and more bacteria are prone to migrate toward the NAPL source, which is also their carbon source, than the normal bacteria. Pseudomonas putida $\mathrm{F} 1$ is one of the five bacterial species that shows strong chemotactic response to and degrades toluene, benzene, ethylbenzene etc. (Parales et al., 2000), and is widely used to study chemotaxis. Besides, chemotaxis is proven to be essential to the formation of biofilms at the interface of different soil layers as well as between NAPL and water in the soil for several different microbial species (Pratt, 1998, O’Toole, 1998 and Prigent, 1999).

\subsection{Bacteria in Porous Media}

\subsubsection{Bacterial Motility in Porous Media}

Bacterial migration is restricted by the limited pore spaces within porous media. As shown in Figure 2.4, in order to accomplish the same linear displacement in bulk aqueous solution from A to B, the actual bacterial swimming trajectory may be elongated in the porous matrix. At the same time, an individual run of a bacterium is often interrupted by the interaction with surfaces of soil grains, and the turn angles are also restricted by the narrow spaces. In fact, for the wild type of $E$. coli, $\mathrm{HCB} 1$, its single run time between two consequent tumbles lasts approximately 1 second (Liu and Papadopoulos, 1995), and the mean swimming speed of is $22.8 \mu \mathrm{m} / \mathrm{s}$ (Lewus and Ford, 2001), so the bacterial original swimming pattern can be frequently changed in the pores with comparable sizes as 
bacterial run length. Berg and Turner (1990) found that the bacterial motility in $10 \mu \mathrm{m}$ capillary tubes was larger than that in $50 \mu \mathrm{m}$ ones, because the small capillary size geometrically restricted the bacterial turning angle, and also helped align the cell bodies along the axis of the tube. Chen and his coworkers (1998) also supported this experimental observation from the mathematical aspect.
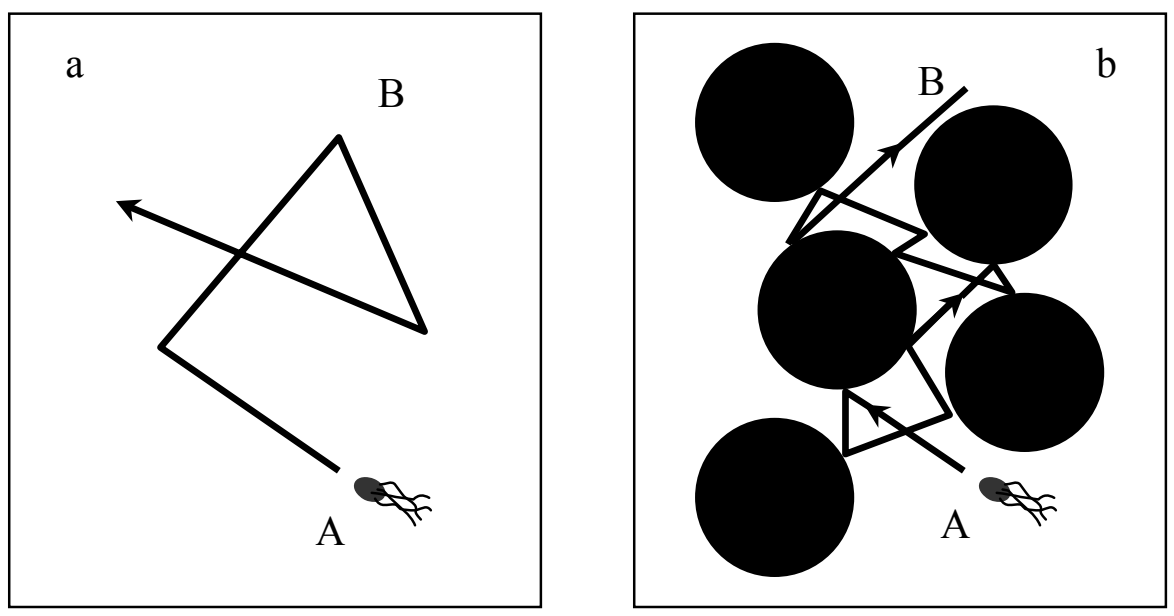

Figure 2.4 Schematic of swimming trajectories of $E$. coli in (a) bulk aqueous solution and (b) porous medium. AB represents the same linear displacement of bacterial movement.

\subsubsection{Bacterial Chemotaxis to NAPL in Porous Media}

As stated in the Motivation section and shown in Figure 1.1, NAPL residuals are very hard to be removed from fine-grained porous media, and traditional methods of environmental remediation are too costly in terms of money, energy and time to deal with NAPL contaminations in groundwater system (Liu et al., 2005 and Boopathy, 2000). Therefore, people turn their attention to bioremediation. Bioremediation is a biological way to approach environmental contamination, and it is cheaper and easier to operate 
than the traditional methods, such as catalytic metal particles, excavation etc. (Langwaldt and Puhakka, 2000). The mechanism of bioremediation is to employ biological forces, such as bacteria, enzymes and plants, to consume and/or degrade the contaminants, and turn them into harmless matters. However, bioremediation is not able to function well enough in heterogeneous soil, which consists of soil layers with different permeabilities. Bacteria from bioremediation injection prefer to swimming along the groundwater flow in both high permeable (upper) and low permeable (lower) soil layers, while NAPL is usually trapped in the low permeable layer with small pore sizes. As a result, the majority of bacteria travel along with groundwater, and only a small fraction of injected bacteria functions their ability of degradation in lower soil layer, thus traditional bioremediation is not very efficient (Figure 2.5).
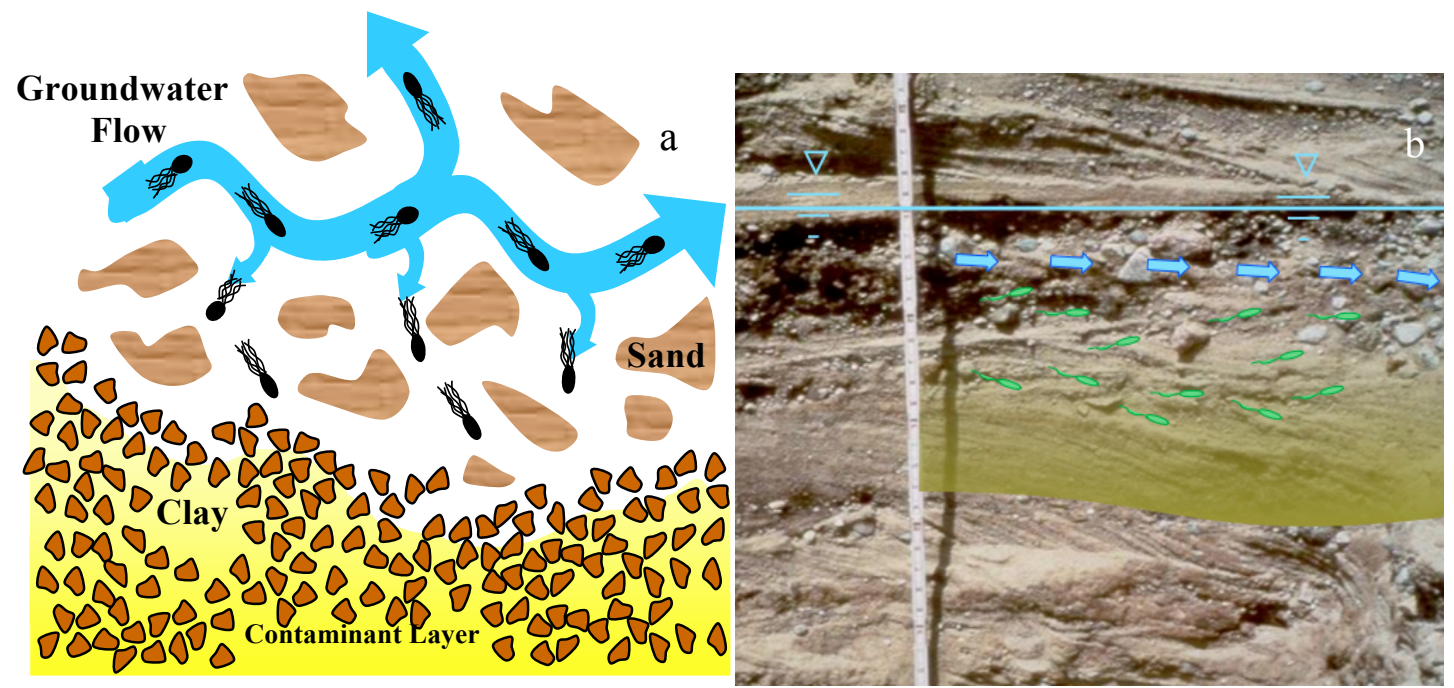

Figure 2.5 (a) Schematic representation of bacterial migration in a heterogeneous aquifer (adapted from Kusy, 2005). (b) Same schematic representation in a natural heterogeneous aquifer (modified from LeBlanc et al., 1991). The blue arrows show the preferential pathway for bacteria (green) in the high permeable soil layer, and the area of yellow shaded region indicates the 
chemical distribution in the low permeable layer.

Chemotaxis might improve bioremediation in the scenario mentioned above. In brief, it is because chemotactic bacteria are able to respond to the concentration gradients of specific external chemicals in its vicinity, and to migrate toward favorable conditions or away from unfavorable conditions. The low permeable soil layer has a higher concentration of NAPL component, which is favored by chemotactic bacteria like Pseudomonas putida F1, so the bacterial population is expected to increase in the contaminant layer (Figure 2.5), and thus chemotactic bacteria should be able to improve the efficiency of bioremediation.

Bacterial chemotaxis in bulk aqueous solution was well characterized in different laboratory-scale studies with or without flow (Adler, 1966; Mesibov et al., 1973; Marx and Aitken, 2000; Parales et al., 2000; McClaine and Ford, 2002; Law and Aitken, 2005; Lanning et al., 2004, 2008). Capillary assays (Mesibov et al., 1973), stopped-flow diffusion chamber assays (Ford et al., 1991), agarose plug assays (Parales et al., 2000) and T-sensor assays (Lanning et al., 2008) could be representative in the series of studies on bacterial chemotaxis in bulk aqueous media. All these studies successfully quantified chemotactic accumulation in very short time. However, this microbial phenomenon in porous media, which is truly critical to the understanding and application of bacterial chemotaxis in contaminated groundwater system, was only recently focused on. Some experiments were conducted to observe chemotaxis to organic chemicals specifically in a flow-free and porous environment. Bacterial chemotaxis to NAPL compound naphthalene was quantified in a modified capillary filled with glass beads (Pedit et al., 2002). Olson et al. (2004) reported a strong chemotactic response of Pseudomonas putida 
F1 to trichloroethylene (TCE) in a glass bead column by magnetic resonance imaging. On the other hand, it was demonstrated that convective flow influenced the effect of chemotaxis on the transverse direction in homogenous and heterogeneous porous media on multiple scales, which included microfluidic porous T-sensor (Long and Ford, 2009), packed column (Wang et al., 2008 and 2009) and two-dimensional flow-through microcosms (Bauer et al., 2008; Strobel et al., 2011). All these experimental approaches drew similar conclusions that bacterial chemotaxis was still significant under slow convective flows. However, all these conclusions were based on the results from aqueous-phase attractants, whereas natural systems typically have nonaqueous phase liquid (NAPL) contaminants. Conventional scales like packed column and microcosm were too hard to include a second liquid phase chemical in the experimental process due to various reasons, such as nonuniform packing, no direct visualization etc. Microscale techniques like microfluidics allows delicate manipulation on small amount of liquid, and can be used to study NAPL problems in an aqueous surrounding. In the next section, I will introduce the microfluidic designs used for study of bacterial chemotaxis, especially for study of chemotaxis phenonmenon in presence of NAPL.

\subsection{Application of Microfluidics in This Study and Related Research}

Microfluidic devices have proven to be effective tools in a wide range of scientific fields, (Whitesides, 2006), and have some ideal features for the study of bacterial chemotaxis. Microfluidic devices allow accurate fabrication of microscopic scale structures and precise manipulation of very small amounts of fluid, which is ideal for the study of NAPL contaminants in pore-scale geometries that are representative of the natural aquifer environment. Moreover, the microscale channel operates under laminar 
flow in most scenarios, so that the concentration gradient of attractant is smooth and mathematically predictable. Its absolute transparency and small size allow direct and quantitative measurement of the bacterial distributions inside the device under a microscope.

\subsubsection{Heterogeneous Microfluidic Device (H- $\mu$ Chip)}

Amongst the earlier studies, microfluidic devices with pore-scale structure were created to study colloid transport (Wan and Wilson, 1994a and 1994b; Auset et al., 2004). Gradually, a variety of microfluidics designs were applied to the study of bacterial chemotaxis with convective flow. Under flow conditions, parallel-flow devices were among the first approaches to generate steep and steady concentration gradients and assess chemotaxis by the extent of transverse bacterial movement towards the chemoeffector stream (Mao et al., 2003; Lanning et al., 2008; Englert et al., 2009). Long and Ford (2009) observed bacterial chemotaxis in a direction transverse to flow under fluid velocities typical of groundwater flow rates in a T-shaped homogeneous porous channel with a staggered array of cylindrical posts. Singh and Olson (2012) created a heterogeneous porous microfluidic device to study transverse chemotaxis, and an increase of chemotactic bacterial population was detected in the low permeable regions containing attractant. All the previous studies successfully detect the effect of bacterial chemotaxis in porous structure under groundwater flow rates; however, they also all treated the contaminants as aqueous phase chemicals other than organic phase ones. A two-phase heterogeneous porous system is more representative of the actual NAPL contaminated sites. Wan and coworkers published several studies of bacterial transport in two-phase systems with water and air in the microfluidic device (Wan, 1994a and Wan, 
1994b), and many researchers continued with this topic to study unsaturated (liquid/gas interfaced) porous media at different scales (Crist, 2004, Garguilo, 2008, Powelson, 2001, Sirivithayapakorn, 2003, Chen, 2009). Wang and coworkers (2012) successfully developed a single-pore microfluidic device to quantify the chemotaxis effect in the vicinity of a stable NAPL/water interface. Although this design mimicked the different pore scales for contaminant source and bacterial pathway, it was still not a porous medium. A more complex experimental platform of microfluidics (Figure 2.6) was developed originally by Lanning (2004) in order to mimic the heterogeneous NAPL contamination condition illustrated in Figure 1.1 to a greater extent. The advantage of this microfluidic device over the "single-pore" one is that it captures some of the essential features of the contaminated soil: soil heterogeneity and NAPL entrapment. It achieves a microscopic level pore structure, which consists of a high permeable channel adjacent to a low permeable porous network with NAPL ganglia. In addition, the NAPL sources in the low permeable region are trapped in a predictable manner for quantitative analysis and evaluation using mathematical models. The heterogeneous microfluidic device $(\mathrm{H}-$ $\mu$ Chip) used in this study was modified from this version with a finer porous network, and was used to study bacterial chemotaxis in response to the attractant sources. 


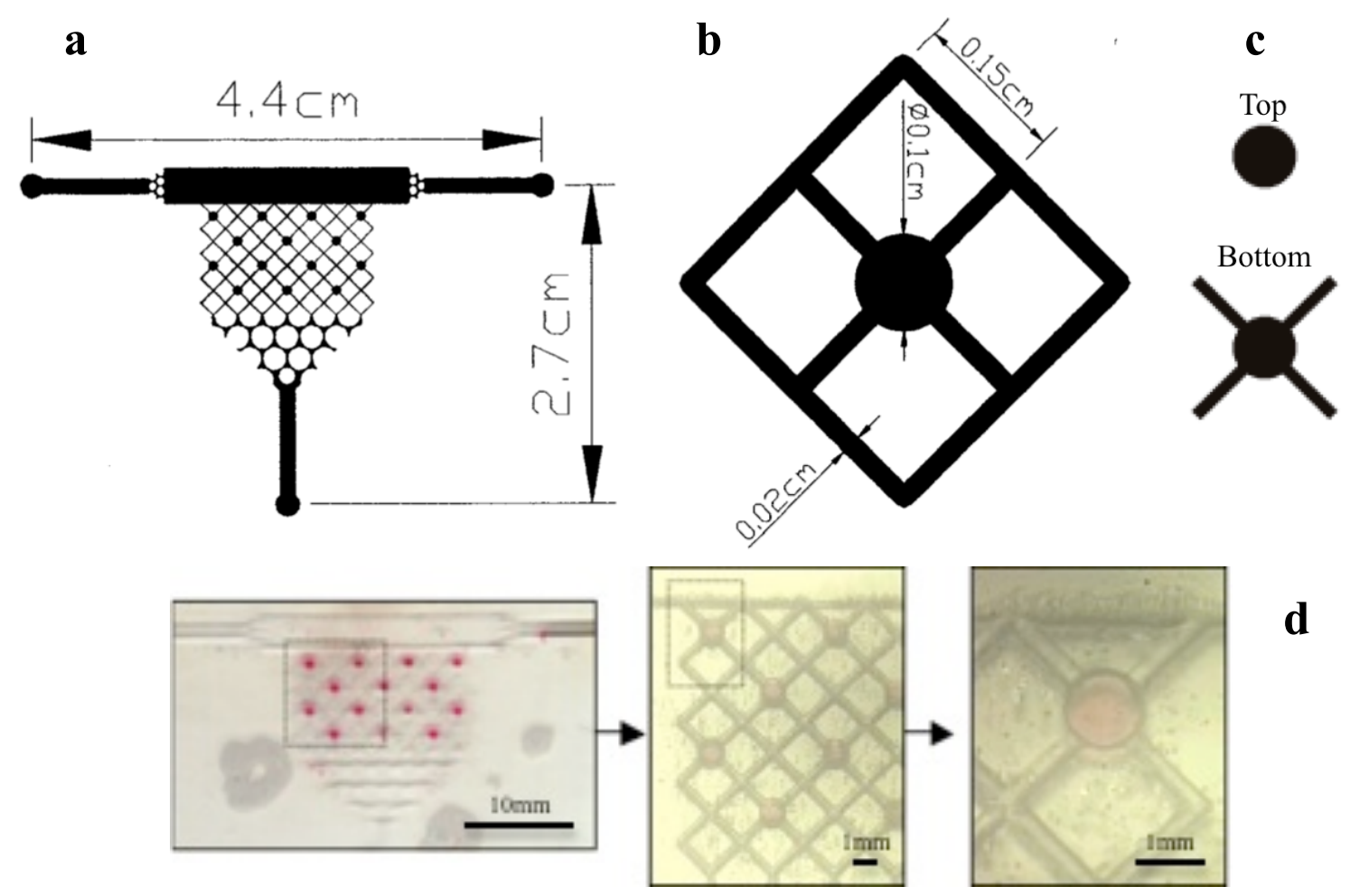

Figure 2.6 Design of the original porous microfluidic device. (a) The overall diagram illustrates the wide horizontal channel as the macropore with high permeability and the network of micropores as the region with low permeability. (b) A more detailed diagram of the low permeable region shows the dimensions of the channels and ganglia. The depth of the straight pores is approximately $100 \mu \mathrm{m}$, and that of the ganglia is approximately $200 \mu \mathrm{m}$. (c) The detailed diagram shows the patterns etched away from the top and bottom glass pieces in the low permeable region. (d) The toluene droplets have been dyed red and are trapped within the circular ganglia of the micromodel network (adapted from Lanning, 2004).

\subsubsection{Convection-Free Gradient Generator (CF- $\mu$ Chip)}

The quantification of chemotaxis is always a challenge. Chemotaxis was proved to be useful to bioremediation by different literatures qualitatively, but not many studies were done to analyze chemotaxis quantitatively. Its quantification is characterized by two 
parameters, chemotactic sensitivity coefficient $\chi_{0}\left[\mathrm{~m}^{2} / \mathrm{s}\right]$ and chemotactic receptor constant $K_{c}[\mathrm{~mol} / \mathrm{L}]$. The chemotactic sensitivity coefficient represents the redirected bacterial movement resulted from the chemotactic response to a certain kind of chemical, and the chemotactic receptor constant represents the bacterial propensity to bind the chemoattractant with the chemotactic receptors (Ford and Harvey, 2007). Mathematical models on diffusion and advection were applied to evaluate the values of the parameters. In order to provide precise measurement, the models need to be simplified as much as possible, so that chemotaxis can be the main or even the only variable that affects the final results. Therefore, an advection-free system with linear attractant concentration gradient at steady state is preferred for both experimental observation and computational fitting.

Multiple approaches were developed to produce time-invariant concentration profiles of attractant without convective flow, and they all basically shared the same operational principle, which was to utilize a hydrogel layer or porous membrane to connect the channels of attractant and bacteria (Diao et al., 2006; Wu et al., 2006; Cheng et al., 2007; Kalinin et al., 2009; Ahmed and Stocker, 2010). The hydrogel and the porous membrane obstructed advection from the testing channel, and helped to establish a steady concentration gradient of the chemical due to pure diffusion. Since there was no interference of convective flow, these studies of bacterial chemotaxis in response to a steady concentration gradient under a flow-free condition could be used to evaluate the bacterial motility and chemotaxis parameters, which are the chemotactic sensitivity coefficient and chemotactic receptor constant. However, all of these attempts were still not precise to create a predictable linear gradient, because the interface between the 
aqueous phase and the hydrogel $/ \mathrm{membrane}$, and the properties of hydrogel $/ \mathrm{membrane}$ itself were subject to unpredictable changes. These changes added some unnecessary complexity into the chemical diffusion and bacterial interaction with material surface, and made it difficult to obtain accurate values of chemotaxis parameters from mathematical modeling. A microfluidic palette design (Atencia et al., 2009) achieved multiple flow-free concentration profiles of attractants in one aqueous phase in a microscale circular chamber by the precise pressure balances among the chamber inlets, which could be used to study the bacterial sensitivity surrounded by different attractant sources, or bacterial response to a transient environmental change, etc. Atencia et al. (2012) developed the prototype of the first convection-free gradient generator (CF$\mu$ Chip), which uses the mechanism of pressure balance from the microfluidic palette design and the knowledge from the previous methods, to create a well-defined gradient without flow in a straight channel that is amenable to mathematical description.

Another critical factor that the previous studies did not take account into was the coupling effect of chemotactic sensitivity coefficient and chemotactic receptor constant. Different combinations of the values of these two parameters could generate similar chemotactic effect, so an imprecise measurement of one parameter would lead to an incorrect value of the other. Measurement for these two parameters has been quite a challenge, especially for the chemotactic receptor constant. This study used a modified design from Atencia's CF- $\mu$ Chip and successfully eliminated the coupling effect by changing the attractant concentration. 


\subsection{Bacterial Fluorescent Labeling and Imaging}

An alternative data collecting and analytical method was developed to replace the conventional and time consuming counting method on bacterial density. Generally speaking, bacterial density was expressed as certain signal intensity, like light intensity, instead of bacterial counting number. Several previous studies adopted similar methodology and successfully recorded bacterial distribution according to chemotaxis. Ford and coworkers (1991) constructed stopped-flow diffusion chamber assays (SFDC) and read the chemotactic bacterial density as their light intensity from phase contrast under bright field; same method was used in the single-pore microfluidic assay by Wang and coworkers (2012). However, light intensity reading had big background noise from device materials, dust etc. Sherwood and coworkers (2003) used immunomaganetic tracers to label bacteria and magnetic resonance imaging (MRI) to detect the bacterial distribution in a glass-beads packed column; this method reduced background noise to a great extent, but the bacterial movement in the column could not be directly seen.

Fluorescence labeling could reduce background noise and allow visualization of bacterial population distribution instantaneously under the microscopes.

The transformation of green fluorescent protein into E. coli bacterial strains is a well-understood case. A common method is to use the arabinose operon (Hanahan, 1983). As shown in Figure 2.7, the single gene for GFP is embedded into the DNA code of the plasmid for arabinose operon. Arabinose molecule interacts with the regulatory protein $\operatorname{araC}$, which sits on the $\mathrm{P}_{\mathrm{BAD}}$ promoter, and consequently changes the protein's conformation. This change facilitates transcription of the gene by RNA polymerase, and thus GFP is transcribed. Ultra violet (UV) light exposure excites GFP molecule, and 
cause the protein to glow in green fluorescence. GFP transformation allows live observation.
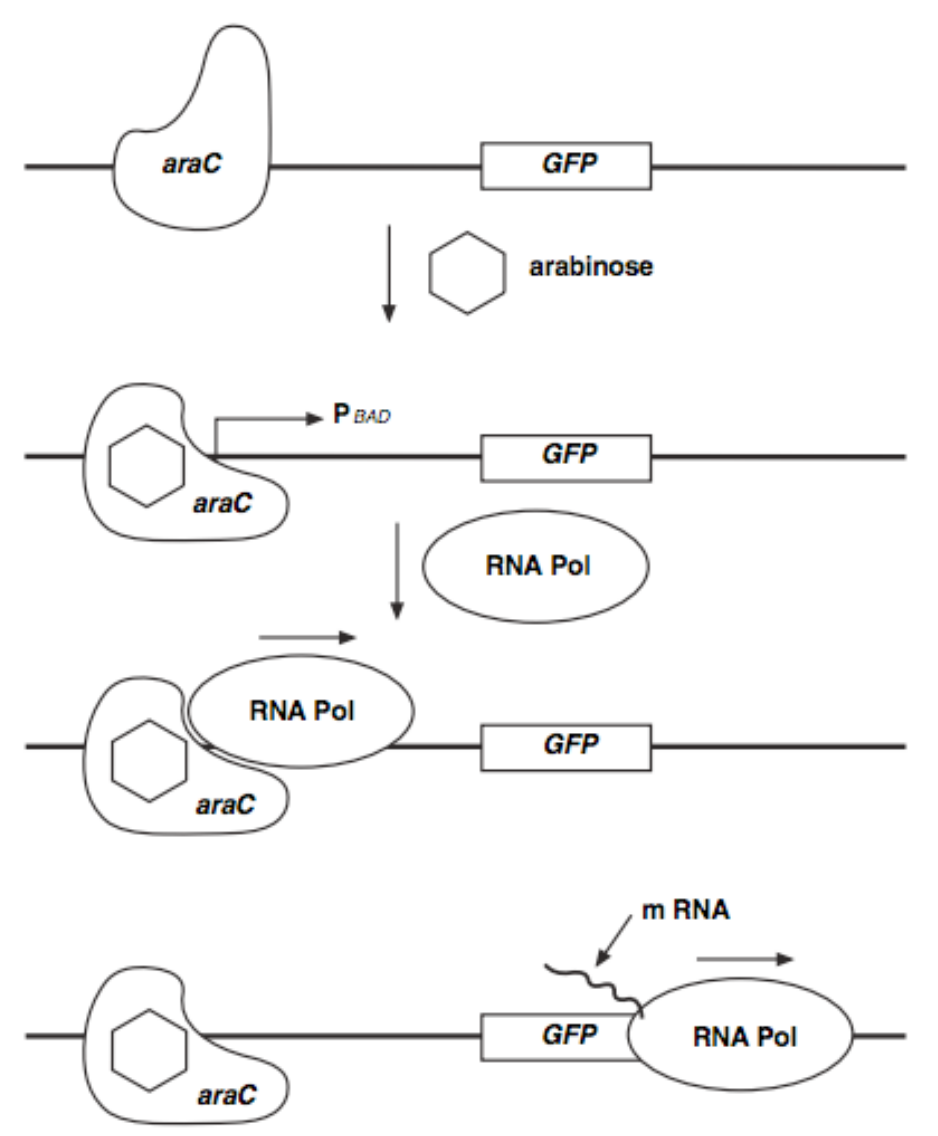

Figure 2.7 Schematic of green fluorescence protein expression through arabinose catabolism.

Other live cell fluorescence stains are also available. Membrane dye N-(3Triethylammoniumpropyl)-4-(6-(4-(Diethylamino) Phenyl) Hexatrienyl) Pyridinium Dibromide (abbreviated FM 4-64) is a good example. FM 4-64 is nontoxic to cells and is believed to bind the outer leaflet of the plasma membrane where this dye becomes intensely fluorescent (Haugland, 1996). This method of labeling has been widely used to selectively visualize plasma membrane of a variety of individual cells including bacteria 
(Vida and Emr, 1995), and allows live observation of cellular activities. Because chemoreceptors are transmembrane proteins embedded in the inner membrane (Stock and Mowbray, 1995), we do not expect any interference between the FM 4-64 membrane staining and the chemotaxis cascade pathway.

\subsection{Summary of the Experimental Design}

In this study, $P$. putida $\mathrm{F} 1$ and its nonchemotactic mutant $P$. putida $\mathrm{F} 1 \mathrm{CheA}$ were used as the study model. E. coli $\mathrm{HCB} 1$ and $\mathrm{HCB} 437$ (smooth-swimming mutant) were also tested for comparison. Microfluidics was chosen as the method to study bacterial chemotaxis due to its advantages as mentioned in Section 2.4. Two different microfluidic designs were used: the heterogeneous microfluidic device (H- $\mu$ Chip) and the convectionfree gradient generator (CF- $\mu$ Chip), as shown in the next chapter Materials and Methods. H- $\mu$ Chip was used to study bacterial chemotaxis in a heterogeneous porous matrix design with residual NAPL ganglia, and CF- $\mu$ Chip was used to measure relatively accurate values of key chemotaxis parameters.

For the H- $\mu$ Chip study, one bacteria/attractant pair was tested. Two flow rates, 0.25 and $5 \mathrm{~m} / \mathrm{d}$, were chosen to study the influence of flow rate on the chemotaxis effect. They are within the range of typical groundwater flow rates from 0.15 to $15 \mathrm{~m} / \mathrm{d}$ (Harter, 2003). The three groups of variables in the $\mathrm{H}-\mu$ Chip experimental design are summarized in Table 2.1. Representative publications relative to this study are summarized in Table 2.2; $\sqrt{ }$ means that specific work contains this feature in its study, and $\mathrm{H}-\mu \mathrm{Chip}$ experiment is also compared with these works in this table. 
Table 2.1 The test variables in the $\mathrm{H}-\mu$ Chip experiments

\begin{tabular}{lll}
\hline Bacterial strain & NAPL component & Flow rate $(\mathrm{m} / \mathrm{d})$
\end{tabular}

P. putida F1 CheA ${ }^{\mathrm{a}}$

Toluene

${ }^{\mathrm{a}}$ This is the nonchemotactic bacterial control.

Table 2.2 Comparisons among the H- $\mu$ Chip study and previous representative works

\begin{tabular}{|c|c|c|c|c|c|c|c|}
\hline & Chemotaxis & NAPL & $\begin{array}{l}\text { Two } \\
\text { phase }\end{array}$ & $\begin{array}{c}\text { Porous } \\
\text { Medium }\end{array}$ & Heterogeneity & $\begin{array}{l}\text { Convective } \\
\text { flow }\end{array}$ & $\begin{array}{c}\text { Quantitative } \\
\text { Analysis }\end{array}$ \\
\hline $\begin{array}{l}\text { Wan et al. } \\
1994\end{array}$ & & & $\sqrt{ }$ & $\sqrt{ }$ & & $\sqrt{ }$ & $\sqrt{ }$ \\
\hline $\begin{array}{l}\text { Pedit et al. } \\
2002\end{array}$ & $\sqrt{ }$ & $\sqrt{ }$ & & $\sqrt{ }$ & & & $\sqrt{ }$ \\
\hline $\begin{array}{l}\text { Olson et } \\
\text { al. } 2004\end{array}$ & $\sqrt{ }$ & $\sqrt{ }$ & & $\sqrt{ }$ & & & $\sqrt{ }$ \\
\hline $\begin{array}{l}\text { Long and } \\
\text { Ford } 2009\end{array}$ & $\sqrt{ }$ & & & $\sqrt{ }$ & & $\sqrt{ }$ & $\sqrt{ }$ \\
\hline $\begin{array}{l}\text { Wang and } \\
\text { Ford } 2009\end{array}$ & $\sqrt{ }$ & & & $\sqrt{ }$ & $\sqrt{ }$ & $\sqrt{ }$ & $\sqrt{ }$ \\
\hline $\begin{array}{l}\text { Strobel et } \\
\text { al. } 2011\end{array}$ & $\sqrt{ }$ & & & $\sqrt{ }$ & & $\sqrt{ }$ & $\sqrt{ }$ \\
\hline H- $\mu$ Chip & $\sqrt{ }$ & $\sqrt{ }$ & $\sqrt{ }$ & $\sqrt{ }$ & $\sqrt{ }$ & $\sqrt{ }$ & $\sqrt{ }$ \\
\hline
\end{tabular}

For the CF- $\mu$ Chip study, the bacterial strains were divided into two groups (see Table 2.3). One was $P$. putida $\mathrm{F} 1$ and its nonchemotactic mutant $P$. putida $\mathrm{F} 1 \mathrm{CheA}$. The 
attractant for the first bacterial group was toluene dissolved $10 \%$ random motility buffer (RMB), and the two test concentrations were 0.2 and $2 \mathrm{mM}$. The other group was $E$. coli HCB1 and E. coli $\mathrm{HCB} 437$. $\mathrm{HCB} 437$ is a smooth swimming mutant and served as a nonchemotactic control; the attractant was $\alpha$-methylaspartate, and the two test concentrations were 0.3 and $3 \mathrm{mM}$ in $10 \% \mathrm{RMB}$. The three groups of variables in the CF$\mu$ Chip experimental design are summarized in Table 2.3.

Table 2.3 The test variables in the CF- $\mu$ Chip experiments

\begin{tabular}{lcc}
\hline Bacterial strain & Attractant & Attractant Concentration (mM) \\
\hline P. putida $\mathrm{F} 1$ & Toluene in $10 \% \mathrm{RMB}$ & 0.2 \\
P. putida $\mathrm{F} 1 \mathrm{CheA}^{\mathrm{a}}$ & & 2.0 \\
& & \\
\hline & & 0.3 \\
E. coli $\mathrm{HCB} 1$ & $\alpha-$ methylaspartate & 3.0 \\
E. coli $\mathrm{HCB} 437^{\text {a }}$ & & \\
& & \\
\hline
\end{tabular}

\footnotetext{
${ }^{\mathrm{a}}$ This is the nonchemotactic bacterial control.
} 


\section{MATERIALS AND METHODS}

\section{ChAPTER 3}

Materials ANd Methods

\subsection{Introduction}

This chapter describes the protocols used in this study, including preparation of bacteria/attractant system, staining, microfluidic fabrication, and image analysis. It details the methods used to correlate bacterial density, optical density (OD), and fluorescence intensity. It also provides the rationale about selection of mathematical modeling equations, which are used to support experimental observations on bacterial chemotaxis, and to quantify the results with accurate parameters. 


\subsection{Preparation of Bacterial Strains and Attractants}

Two bacterial and attractant systems were used in this study. One bacteria/attractant pair was P. putida and toluene. P. putida F1, the wild type, was obtained from C. S. Harwood (Parales et al., 2000) and nonchemotactic mutant $P$. putida F1 CheA from R. E. Parales (Liu et al., 2009). An aliquot (300 $\mu \mathrm{L}$ for P. putida) of frozen stock at $-80^{\circ} \mathrm{C}$ was used to inoculate $100 \mathrm{~mL}$ of growth media in a sealed 200 $\mathrm{mL}$ glass bottle. The Modified Hutner's Mineral Base for $P$. putida contained basic components of Hutner's Mineral Base (Olson, 2004), $600 \mu \mathrm{g}$ LB powder (Miller, ACROS LB Broth), with $30 \mu \mathrm{L}$ toluene (Sigma-Aldrich, HPLC grade) injected into the gas phase. Oxygen (GT\&S, oxygen compressed USP) was injected at 5 psi for 10 minutes to ensure a sufficient supply. Bacteria were cultured on the Thermo Scientific Incubated and Refrigerated Shaker (Model MaxQ 4000) with a rotation rate of $150 \mathrm{rpm}$ at $28^{\circ} \mathrm{C}$. When the optical density (measured at $590 \mathrm{~nm}$ in Beckman Coulter, DU 730) reached 0.80 (corresponding to the mid-exponential phase of growth), bacteria were harvested and filtered from the culture media with a $0.22 \mu \mathrm{m}$ membrane filter (Millipore, GSWP14250), and resuspended in 10\% Random Motility Buffer (RMB, $11.2 \mathrm{~g} \mathrm{~K}_{2} \mathrm{HPO}_{4}$, $4.8 \mathrm{~g} \mathrm{KH}_{2} \mathrm{PO}_{4}$, and $0.029 \mathrm{~g}$ EDTA per liter of deionized water) to a density similar to the one when harvested.

P. putida F1 was dyed with the vital membrane stain FM 4-64 (Invitrogen) at a concentration of $5 \mu \mathrm{g} / \mathrm{mL}$ for 10 minutes and F1 CheA was dyed with $100 \mathrm{mg} / \mathrm{L}$ DAPI (Invitrogen) for 30 minutes, and both bacterial suspensions were washed twice before the final use. The movement of stained bacteria was observed under Wide-Field Microscope (Olympus IX-70) and compared visually with the unstained bacteria. There was no 
observable change in motility between the unstained and FM 4-64 stained bacteria, as the run length and tumbling frequency appeared to be the same between the two groups, and both unstained and stained bacteria swam actively by visual comparison. Quantitative analysis was done and also showed that the dye FM 4-64 itself did not affect the bacterial motility (Appendix E). The two bacterial suspensions with different dyes were then mixed for later experimental use. The bacteria suspension was also mixed with $1.2 \mathrm{mM}$ sodium nitrate (Sigma-Aldrich, ACS reagent), an electron acceptor to maintain bacterial motility (Bauer et al., 2008). Toluene (Sigma-Aldrich, HPLC grade) aqueous solution was also prepared for the measurement of chemotaxis parameters, and the toluene concentrations were 0.2 and $2 \mathrm{mM}$ in $10 \% \mathrm{RMB}$.

The other bacteria/attractant pair was $E$. coli and $\alpha$-methylaspartate. E. coli HCB1 was selected because it presented a strong chemotactic response to $\alpha$-methylaspartate (Lanning et al., 2008), which is a non-metabolizable analog of aspartate. The preparation of the frozen aliquots for E. coli $\mathrm{HCB} 1$ (wild type) and HCB437 (smooth swimming mutant), which were generously provided by Dr. Howard Berg at Harvard University (Wolfe et al., 1987), was the same as that for the $P$. putida strains. HCB437 mutant could not perform the tumble function, so the bacteria were unable to adjust their swimming direction in a changing chemical environment, and therefore, could not exhibit chemotaxis. E. coli HCB1 was genetically tagged with green fluorescent protein (BioRad, pGLO bacterial transformation kit), and HCB437 was treated with the same DAPI staining protocol as $P$. putida F1 CheA. A $100 \mu \mathrm{L}$ volume of E. coli strains were cultured aerobically in $50 \mathrm{~mL}$ TB media [0.50 g tryptone (BD BactoTM) and $0.25 \mathrm{~g} \mathrm{NaCl}$ (Fisher Scientific Certified A.C.S.)]. $100 \mu \mathrm{L}$ of $10 \mathrm{mg} / \mathrm{mL}$ ampicillin (Acros, 99\%) and $1 \mathrm{~mL}$ of 
$100 \mathrm{mg} / \mathrm{mL} \mathrm{L}-(+)$-arabinose (Acros, 99+\%) were added into HCB1's medium to provide selective pressure for plasmid-containing and fluorescing bacteria. The $\alpha$-methylaspartate (Sigma-Aldrich) concentrations were 0.3 and $3 \mathrm{mM}$ in 10\% RMB. The harvesting, mixing protocols for E. coli were the same as those for P. putida.

\subsection{Design and Fabrication of the $\mathrm{H}-\mu \mathrm{Chip}$}

The design used for the heterogeneous microfluidic device (H- $\mu$ Chip) is presented in Figure 3.1a, in which the higher permeable region is represented by the horizontal channel above and the lower permeable region/the contaminant layer is represented by the fine cross-hatched pore network below with aligned circular ganglia. Figure $3.1 \mathrm{~b}$ shows a closer view of the fine pore network with one circular ganglion. The dimensions of the channels and network are typical of pores and soil grains within a low permeable layer. The ganglia and the channel/network patterns were etched, by hydrofluoric acid, in two separate borofloat glasses sheets, as shown in Figure 3.1c. 


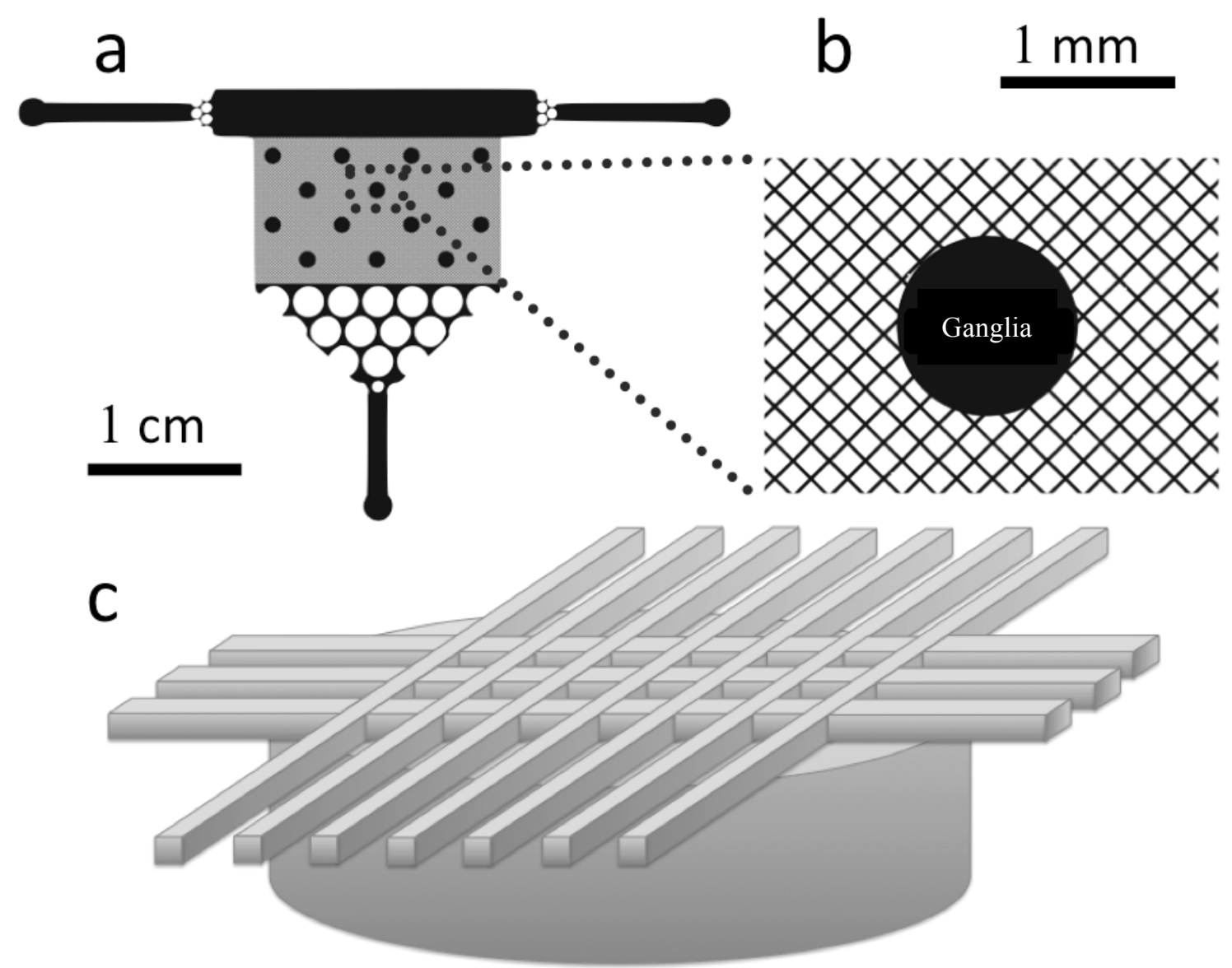

Figure 3.1 (a) Full view of the heterogeneous microfluidic device (H- $\mu$ Chip); the two rounded ends of the horizontal channel are bacterial inlets/outlets, and the third one at the bottom of the device is the NAPL inlet. (b) Zoomed-in view of a part of the fine-grained pore network. Each of the straight channels has a width of $20 \mu \mathrm{m}$. (c) 3D expanded view of the fine-grained network shown in (b).

The microfabrication process followed the photolithography procedure from Roper et al. (2003). First, the photomask was designed. CorelDraw X4 was used to depict the black/white microfluidic design (Figure 3.1a) on an $8.5^{\prime \prime} \times 11^{\prime \prime}$ page. The patterns for one layer were confined to a $5^{\prime \prime} \times 5^{\prime \prime}$ square because these are the dimensions of the glass 
sheet that are available for microfluidic fabrication. White and black colors were chosen for the appropriate features; the black parts on the design represented the areas that were etched away in the glass-etching step. Then the design was transferred onto a mask of transparent film. Figure 3.1 shows the microfluidic design. Dimensions of the main features are as follows: the channel depth was chosen to be $20 \mu \mathrm{m}$, so that the narrow space between channel ceiling and floor did not interfere with bacterial motility (DiLuzio et al., 2005), and ganglia have a depth of $100 \mu \mathrm{m}$ to retain the toluene droplets. Each of the straight channels in the porous network has a width of $20 \mu \mathrm{m}$, which was representative of the actual pore size within a contaminant layer in sandy soil (Hassink, 1993).

The glass etching procedure, illustrated in Figure 3.2, was conducted in the Class 100 clean room in the laboratory of Dr. James Landers in the Department of Chemistry at the University of Virginia. The purpose of glass etching was to create channel patterns of the $\mathrm{H}-\mu$ Chip according to the design. The $5^{\prime \prime} \times 5^{\prime \prime}$ mask was cut from the transparent film and placed on a $1.1 \mathrm{~mm}$ thick Borofloat photomask glass blank (size: $5^{\prime \prime} \times 5^{\prime \prime}$, Telic Co.) with a $530 \mathrm{~nm}$ layer of AZ1518 positive photoresist on a 120nm chrome layer, exposed to collimated UV light (Optical Associates Inc.) at $26 \mathrm{~mW} / \mathrm{cm}^{2}$ for $5 \mathrm{~s}$. After exposure, the glass blank was coated with photoresist developer (AZ@400K 1:4 diluted, Clariant Corp.) for $1 \mathrm{~min}$ and rinsed by deionized water. Then it was placed in chromium photomask etchant (CR-7S, Microchrome Technologies Inc.) until chromium was fully removed from the exposed patterns, after which it was rinsed thoroughly using with deionized water. The glass was then placed in etching solution of $200 \mathrm{~mL} \mathrm{HF}$ and $30 \mathrm{~mL}$ $\mathrm{HNO}_{3}$ with an etching rate of $7 \mu \mathrm{m} / \mathrm{min}$ for $14 \mathrm{~min}$. A $100 \mu \mathrm{m}$ high channel was expected 
to form under these conditions. After etching, the glass was rinsed with excessive amounts of deionized water ( $2 \mathrm{~L}$ of volume for a $5^{\prime \prime} \times 5^{\prime \prime}$ mask) and dried with compressed air. The dimensions of the features on the glass were measured by a highresolution profilometer (XP-1, Ambios Technology). AZ photoresist left on the glass was removed by stripper AZ 300T (Clariant Corp), and chromium was removed by chromium photomask etchant. The etched surface was cleaned by Piranha solution $\left(\mathrm{H}_{2} \mathrm{SO}_{4}: \mathrm{H}_{2} \mathrm{O}_{2} 3: 1\right.$ $\mathrm{v}: \mathrm{v})$ for $30 \mathrm{~min}$.
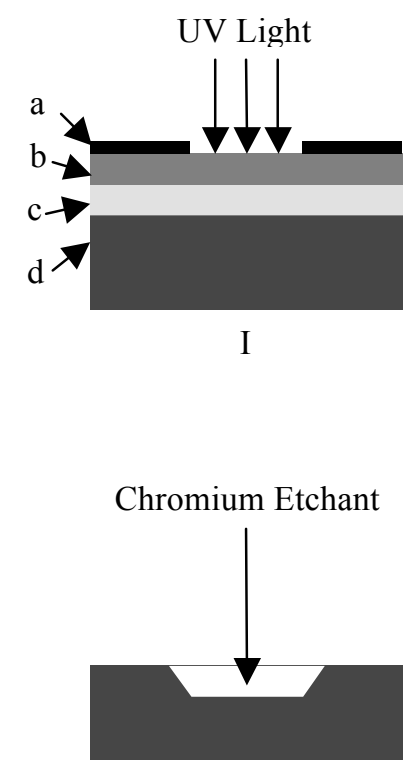

VI

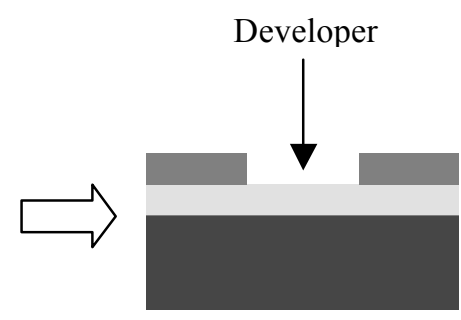

II

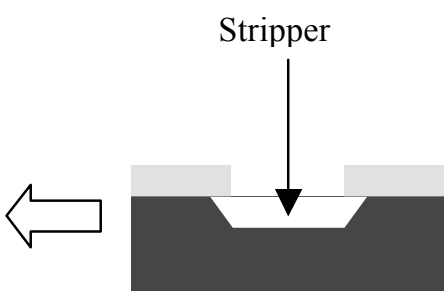

V

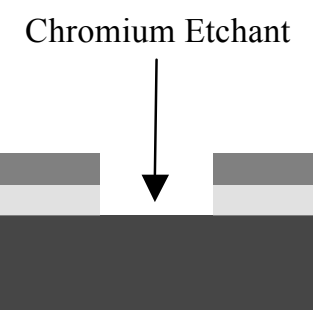

III

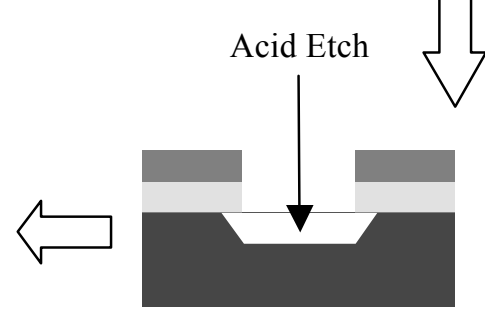

IV

Figure 3.2 The basic steps in the procedure of glass etching. The various layers include (a) the mask, (b) the positive photoresist layer, (c) the chromium layer, and (d) the Borofloat glass. The etching process consists of six steps: (I) UV light exposure, (II) development, (III) chromium removal, (IV) acid etch, (V) photoresist removal by stripper, and (VI) final chromium removal.

As mentioned above, the microfluidic system was comprised of two separate glass layers: the top layer with the macrochannel and pore network and the bottom layer with 
the ganglia. The top layer was drilled with access holes at the three ends of channels (Figure 3.1a) using a 1.1-mm-diameter diamond-tipped drill bit (Crystalite Corp., Lewis Center, $\mathrm{OH})$. The two etched glass layers were irreversibly sealed together by thermal bonding at $690^{\circ} \mathrm{C}$.

The connections to access holes were made of polydimethylsiloxane (PDMS), a kind of elastic polymer after curing. $35 \mathrm{~g}$ PDMS was prepared with a 10:1 (v:v) mixture of its base and curing liquid agent (184 Silicone elastomer, SYLGARD), and subject to vacuum for $45 \mathrm{~min}$ to minimize gas bubbles. The mixture was then poured into an $8 \mathrm{~cm}$ (W) $\times 10 \mathrm{~cm}(\mathrm{~L}) \times 5 \mathrm{~cm}(\mathrm{H})$ plastic box, and put into a $70{ }^{\circ} \mathrm{C}$ oven overnight until PDMS cured to form a solid state. $1 \mathrm{~cm} \times 1 \mathrm{~cm} \times 1 \mathrm{~cm}$ PDMS blocks were cut out and a hole for the connector was made using a stainless needle of GA 21. Then the PDMS blocks were bound at the locations of access holes by plasma binding for $1 \mathrm{~min}$ at high radiation level. A complete H- $\mu$ Chip with the PDMS block connections is shown in Figure 3.3. Needles of GA21 (IV1 PrecisionGuide Needle, BD) were clipped to form $1 \mathrm{~cm}$ long pins, which were used to connect Teflon tubing (Tub $1 / 16 \times .030 \times 5 \mathrm{ft}$, Upchurch Scientific) to the holes in the chip. 


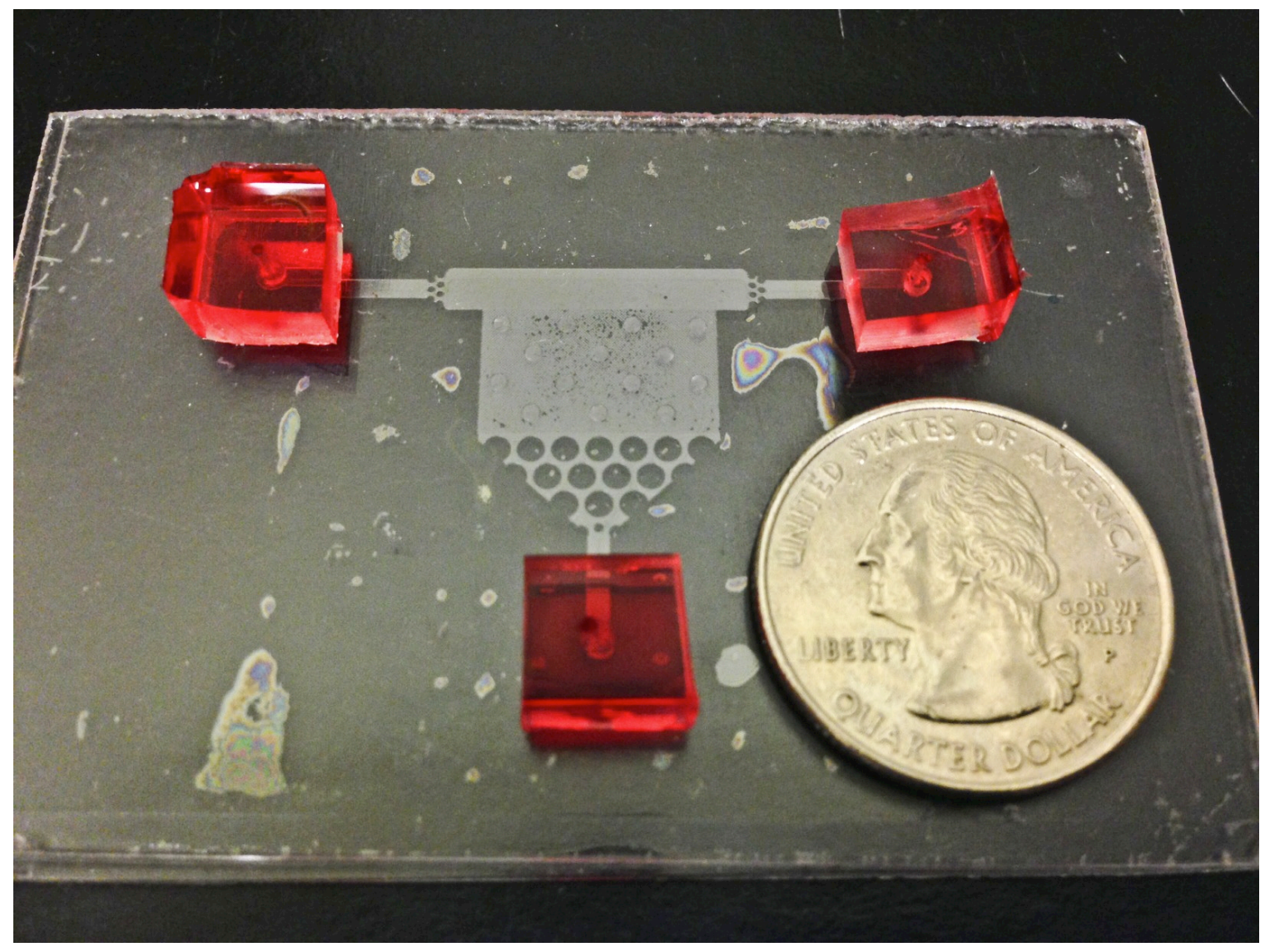

Figure 3.3 A complete H- $\mu$ Chip with the PDMS blocks at the inlets and outlets. The size of the device is comparable to a US quarter. The red color of the PDMS blocks is resulted from the hydrophobic dye Red Oil O in the toluene after the toluene solution contacts the PDMS polymer.

\subsection{Design and Fabrication of the $\mathrm{CF}-\mu \mathrm{Chip}$}

The mechanism of how convection-free gradient generator ( $\mathrm{CF}-\mu \mathrm{Chip})$ worked was well introduced by Atencia and coworkers (2012), as shown in Figure 3.4. Generally speaking, Atencia proposed an equal pressure method to obtain a diffusion-only environment in a microfluidic channel (Figure 3.4). This convection-free gradient generator is composed with multilayers. The top layer (Figure 3.4a) allows aqueous solutions to be injected into its horizontal main channel and flow along side each other. 
The same locations in $\mathrm{x}$ direction are subject to the same pressure. Vias with the identical pressure connect the top layer to the buried channel in the bottom layer (Figure 3.4b), so that the pressures at both ends of the cross channel are equal, and thus achieve the convection-free condition (Figure 3.4c). The cross channel is $40 \mu \mathrm{m}$ high, $600 \mu \mathrm{m}$ wide and $1.5 \mathrm{~mm}$ long. Because the vias are adjacent to the sidewalls of the horizontal channel, the concentration of matter in a via is not affected by flow oscillation and transverse dispersion between the two different flows, and remains unchanged from the injection (Figure 3.4d). Replicate cross channels in the bottom layer can ensure multiple measurements under identical conditions in the same device (Figure 3.4e). 


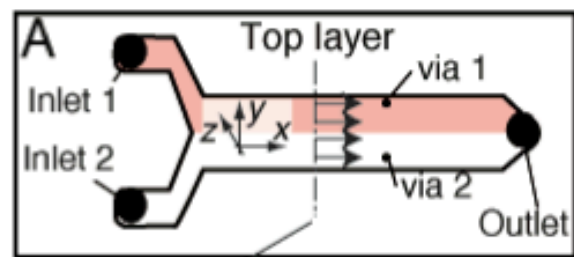

$$
\mathrm{P}=\text { constant }
$$

C

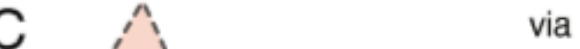

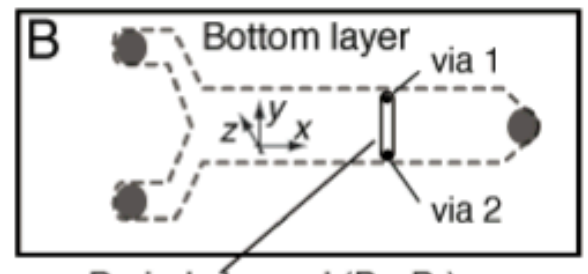

Buried channel $\left(P_{1}=P_{2}\right)$

via 1
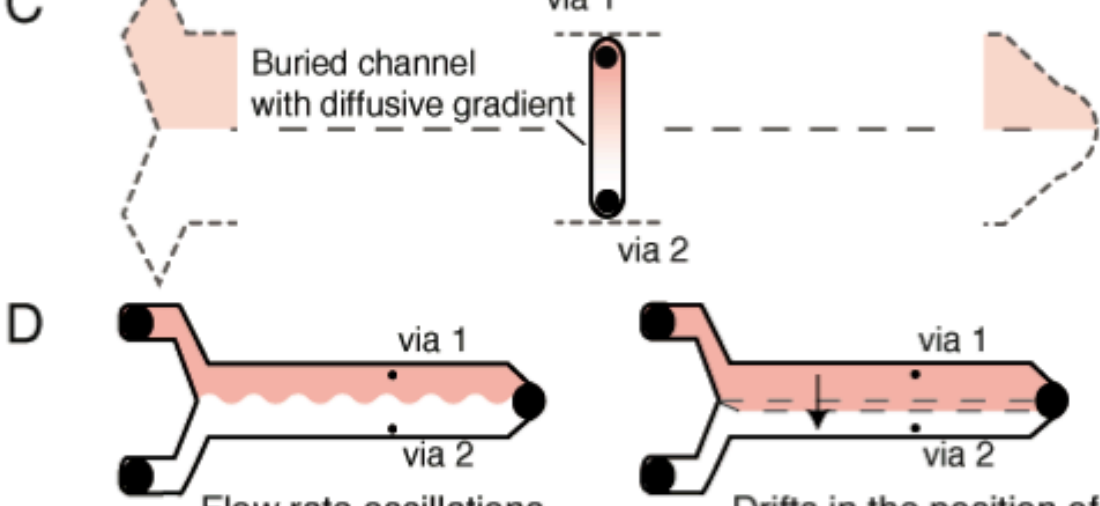

Flow rate oscillations

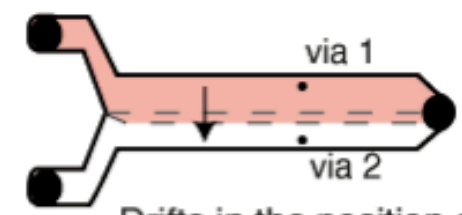

Drifts in the position of the diffusive interface

E

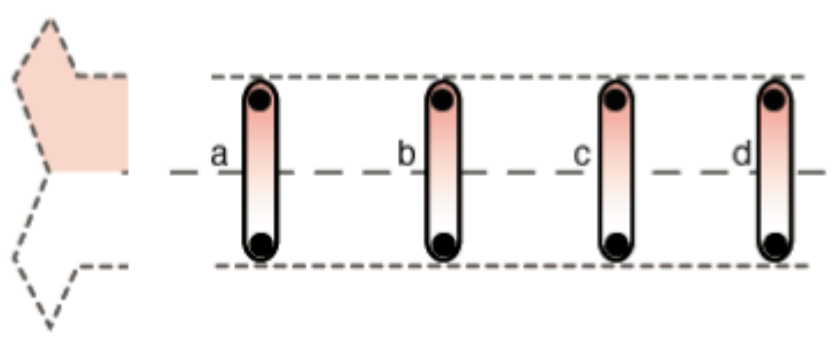

Figure 3.4 Diagram of convection-free gradient generator (Courtesy of Dr. Javier Atencia). (A)

Vias at the same cross section of the horizontal channel (top channel) connect to (B) the two ends of a buried channel in the bottom layer. (C) Two aqueous fluids with different chemical compositions flow parallel to each other in the horizontal channel at same velocity, generating identical pressure at the two symmetric vias, resulting in a diffusion-only linear concentration profile in the buried channel. (D) The vias are away from the diffusive interface between the two fluid streams, and thus the concentration gradient in the buried channel is not affected by the flow oscillation and diffusive region in the horizontal channel. (E) At a desired flow rate, four independent buried channels $(a, b, c, d)$ can achieve the same full concentration gradient of the chemical, and provide replicate conditions for bacteria in the same device. Adapted from Atencia 
et al., 2012.

A new device was developed using PDMS channels based on the same mechanism. The reason why PDMS is used instead of the original material glass is because PDMS has excellent air permeability and can provide sufficient oxygen for bacteria to keep their motility. The new CF- $\mu$ Chip is still composed with three layers.

The centerpiece was made from two pieces of thin cover glasses $(24 \times 50$ Fisher Scientific No. 1.5, 175um thickness) sandwiching a mixture of black ink (Sharpie, Sanford) and super glue (loctite). The black ink was used to stop the background noise. The glue mixture should be put onto the top of one of the cover glasses quickly and evenly. The new assembled centerpiece was perforated with a sand blaster (Airbrasive 6500, s.s. white) to make holes. To facilitate registration and hole-sand-blasting we used a tape stencil with holes designed with Adobe illustrator and cut out with a laser cutter (VersaLASER, Universal Laser Systems Inc., AZ). The material of the other two layers was replaced by PDMS. The glass molds for the PDMS layers were prepared according to the same photolithography protocol for H- $\mu$ Chip. PDMS was cured on top of the mold patterns, and then peeled off for assembling. A convenience of using PDMS to assemble the device is that the bottom layer with the cross channels can be reversibly bonded with the centerpiece by PDMS's natural stickiness, and it can be cleaned and reused easily just by peeling it off from the centerpiece. The top layer was still plasma bonded with the centerpiece. The bacterial suspension and attractant solution were injected into the channel by syringe pump (PHD 2000 Infusion, Harvard Apparatus) and pushed at a constant speed of $1.0 \mathrm{~mL} / \mathrm{h}$, which was comparable to $0.38 \mathrm{~mm} / \mathrm{s}$ in the main channel and 
Reynolds number $\operatorname{Re}=0.28$, which means that the two streams are creeping flows and only diffusion happens on their transverse direction.

\subsection{The Correlation Between Bacterial Density and Fluorescence Intensity}

One conventional way to determine bacterial density is to count individual cells, which is time-consuming and error-prone. Instead, for this analysis, it was assumed that the fluorescence intensity of each cell was similar to each other in the population, thus allowing the light intensity to be correlated with the bacterial concentration in terms of optical density.

GFP tagged E. coli HCB1 was cultured and harvested when its optical density (OD) value at $590 \mathrm{~nm}$ reached to 0.80 . The collected bacteria were filtered and resuspended in $10 \% \mathrm{RMB}$, to form a concentration set of eight from $\mathrm{OD}_{590} 0.1$ to 0.5 , which covered the working $\mathrm{OD}_{590}$ value of 0.4 . Part of the solutions was stained with acridine orange (AO) protocol, and bacterial density was calculated from the counting number of the AO stained cells. With 10\% RMB as control, images of GFP fluorescence from the eight bacterial suspensions were captured by the Wide-Field microscope (Olympus IX-70) in Keck Center of Cellular Imaging at the University of Virginia. Image $\mathrm{J}$ was used to record the fluorescence intensities from the images. Then calibration curves were drawn to relate $\mathrm{OD}_{590}$ values with bacterial densities from $\mathrm{AO}$ counting and also fluorescence intensities. 


\subsection{Image and Data Analysis}

The Wide-Field epifluorescence microscope (Olympus IX-70) was used for all experimental observations and imaging recording with the $2 \times / 0.05$ (Olympus, Plan) and 20×/0.40 objective (Olympus, PlanFI). An arc lamp and dichroic filter (Chroma 51001bs, double coated for TRITC and DAPI) were used to look at both the FM 4-64 (excitation at $515 \mathrm{~nm}$, emission at $640 \mathrm{~nm}$ ) and DAPI (excitation at $345 \mathrm{~nm}$, emission at $455 \mathrm{~nm}$ ) stained P. putida strains, and FITC and DAPI filters (Chroma31013 602 NB) were used for GFP (excitation at $385 \mathrm{~nm}$, emission at $508 \mathrm{~nm}$ ) tagged and DAPI stained E. coli strains. Images were then converted to 8-bit during the import to Image J. The Grouped Stack Projector was used to take the average image from the repeated images at same location, in order to smoothen the curve of bacterial distribution. The data were then normalized for further comparisons.

\subsection{Mathematical Modeling, Computational Fitting and Statistical Analysis}

The first question to ask before choosing the necessary mathematical models is how many spatial coordinates are required to adequately specify the experimental system; to put into other words, should the models be in forms of 1D, 2D or 3D equations? This question can be answered by evaluating the variation of the fluid velocity in the microfluidic device in each dimension and considering its effect on bacterial distribution. In some cases, a gradient in the velocity near the channel wall can lead to the so-called "butterfly effect" (Lanning, 2004).

Weigl (1996) first discussed the effect of a parabolic velocity profile on the shape of the diffusive front of an analyte in microfluidic devices, where the extent of transverse 
diffusion across the channel was related to the channel depth. As the shape of the interdiffusion zone between two streams was similar to a butterfly at a cross-section of a microfluidic channel, this phenomenon was termed the "butterfly effect".

The extent of the "butterfly effect" is quantified by the dimensionless expression in Equation 3.1 (Kamholz, 1999),

$$
\frac{d}{y}=\frac{d}{\sqrt{6 D_{N} t}}=\frac{d}{\sqrt{6 D_{N}\left(z / u_{z, \text { avg }}\right)}}
$$

where $d$ stands for the depth of the channel, and $y$ is the mean diffusion distance using Einstein's equation for diffusion $\left(<y>^{2}=6 D_{N} t\right), D_{N}$ is the diffusion coefficient of the analyte, and the elapsed time of the experiment $t$ equals the total distance traveled down the length of the channel $z$ divided by the average fluid velocity $\left(u_{z, a v g}\right)$.

If the value of the dimensionless expression in Equation 3.1 is significantly smaller than 1, the "butterfly effect" can be neglected. As indicated by the equation, a smaller $d$ could reduce the "butterfly effect". However, if $d$ were so shallow as to be comparable to the bacterial run length, the bacterial motility would be altered. If the distance between the "ceiling" and the "floor" of the channel was smaller than $10 \mu \mathrm{m}$, the bacterial movement could be biased (DiLuzio, 2005). Thus, the height was chosen to be $20 \mu \mathrm{m}$ in order to diminish the impact on bacterial swimming behavior and keep the height sufficiently small to justify a 2D description. The parameter values for $D_{N}$, which for bacteria is the random motility coefficient $\left(\mu_{0}\right)$ and attractant is the diffusivity $\left(D_{a}\right)$, are reported in Table 6.1, the travel length $z$ is $3 \mathrm{~mm}$ for the horizontal channel, $30 \mu \mathrm{m}$

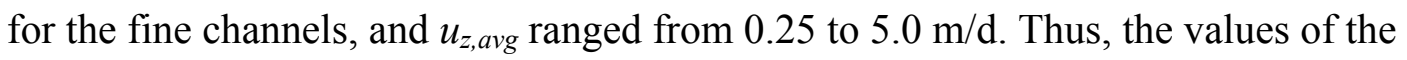
dimensionless expression ranged from 0.01 to 0.08 , and it was assumed that "butterfly 
effect" had a negligible impact on transverse diffusion in the direction of the channel depth. Therefore, a 2D mathematical model was sufficient to simulate the bacterial chemotaxis in the microfluidic device and evaluate the chemotactic properties.

A two-dimensional form of the advection-dispersion governing equation was suitable to describe bacterial transport with the effect of chemotaxis in H- $\mu$ Chip. A twodimensional Brinkman equation is adopted to solve the flow profile in the porous microfluidic device,

$$
\begin{aligned}
& \frac{\rho}{\varepsilon_{p}} \frac{\partial \mathrm{u}}{\partial t}+\nabla \cdot\left[-\frac{\mu}{\varepsilon_{p}}\left(\nabla \mathrm{u}+(\nabla \mathrm{u})^{T}\right)+p \mathrm{I}\right]=-\frac{\mu}{\kappa_{b r}} \mathrm{u} \\
& \nabla \cdot \mathrm{u}=0
\end{aligned}
$$

and $\mathbf{u}$ denotes the flow velocity vector $(\mathrm{m} / \mathrm{s}), \rho$ the fluid density $\left(\mathrm{kg} / \mathrm{m}^{3}\right), \varepsilon_{p}$ the porosity (dimensionless), $\kappa_{b r}$ the permeability of the porous medium $\left(\mathrm{m}^{2}\right), \mu$ the dynamic viscosity (Pa.s), and $p$ the pressure $(\mathrm{Pa})$. The advection-dispersion governing equations that follow are modified from Olson et al. (2008),

$$
\begin{gathered}
\frac{\partial a}{\partial t}=D_{e, a} \frac{\partial^{2} a}{\partial x^{2}}+D_{e, a} \frac{\partial^{2} a}{\partial y^{2}}-\frac{\partial\left(v_{x} a\right)}{\partial x}-\frac{\partial\left(v_{y} a\right)}{\partial y} \\
\frac{\partial b}{\partial t}=D_{e, b} \frac{\partial^{2} b}{\partial x^{2}}+D_{e, b} \frac{\partial^{2} b}{\partial y^{2}}-\frac{\partial\left(v_{x} b\right)}{\partial x}-\frac{\partial\left(v_{y} b\right)}{\partial y}-\frac{\partial\left(v_{C h x} b\right)}{\partial x}-\frac{\partial\left(v_{C h y} b\right)}{\partial y}
\end{gathered}
$$

where

$$
\begin{aligned}
& D_{e, a}=\varepsilon_{p} D_{a} \\
& D_{e, b}=\varepsilon_{p} D_{b}
\end{aligned}
$$




$$
\begin{aligned}
& v_{C h x}=\frac{2}{3} v_{b} \tanh \left(\frac{\chi_{0}}{2 v_{b}} \frac{K_{c}}{\left(K_{c}+a\right)^{2}} \frac{\partial a}{\partial x}\right) \\
& v_{C h y}=\frac{2}{3} v_{b} \tanh \left(\frac{\chi_{0}}{2 v_{b}} \frac{K_{c}}{\left(K_{c}+a\right)^{2}} \frac{\partial a}{\partial y}\right)
\end{aligned}
$$

and $a$ refers to the attractant concentration, $b$ the bacterial concentration, $v_{x}$ and $v_{y}$ the longitudinal and transverse fluid velocity solved from the two-dimensional Brinkman equation, $D_{a}$ the diffusion coefficient for attractant, $D_{b}$ the bacterial random motility, and $D_{e, a}, D_{e, b}$ are their effective values. $v_{C h x}$ and $v_{C h y}$ are the longitudinal and transverse chemotactic velocities describing the convective transport of bacteria caused by chemotaxis in attractant gradients, $\chi_{0}$ the chemotactic sensitivity coefficient and $K_{c}$ the chemotactic receptor constant. The time derivative on the left hand side of Equation 3.3 goes to zero, when the system reaches steady state for the attractant.

The commercial finite-element solver COMSOL Multiphysics 4.3 was used to solve the system of differential equations for the attractant and bacterial concentrations. The setup of conditions and selection of modules are included in the modeling sections in Chapter 4 and 5, and also in appendix. The method of least squares is used to approximate the fitting solution. The best fit in the least-squares sense minimizes the sum of squared residuals, a residual being the difference between an observed value and the fitted value provided by a model. Two-way ANOVA tests were applied to experimental data in bacterial distribution profiles to determine statistically significant differences among various data sets. Averages and standard deviations were estimated from four independent experimental runs for each data set. The tests were done in Prism 5.0a (GraphPad Software Inc.). 


\section{CF- $\mu$ CHIP}

\section{Chapter 4}

CF- $\mu$ CHIP

\subsection{Abstract}

Chemotaxis was proven to be beneficial for the migration of degrading bacteria towards to industrial contaminant sources. Many studies demonstrated the importance of this microbial property under different circumstances; however, few quantitative analyses were done to measure the two essential parameters: chemotactic sensitivity coefficient $\chi_{0}$ and chemotactic receptor constant $K_{c}$. It has been a challenging task to measure these parameters precisely, and no previous studies noticed that the coupling effect between $\chi_{0}$ and $K_{c}$ could prevent researchers from predicting the correct values, nor did they provide any way to solve this problem. This study used a multilayer microfluidic device and created a convection-free channel inside the chip by generating pressure balance at the two ends. Bacterial distribution in the channel was captured in terms of fluorescent 
intensity at steady state, and chemotactic bacterial distribution was compared with nonchemotactic mutant in presence of attractant at different concentrations, and more chemotactic population migrated into the channel at appropriate attractant concentration; the differences were statistically significant with $\mathrm{p}<0.0001$ by two-way ANOVA. The different attractant concentrations helped measure the correct value of $K_{c}$, and then $\chi_{0}$ could be easily determined after fitting the chemotactic patterns with the correct $K_{c}$ in the mathematical models. Both Pseudomonas putida and Escherichia coli strains were used, and the predicted chemotaxis parameters were consistent with the published results and the values derived from related work.

\subsection{Introduction}

The main purpose of this chapter is to present all the work on chemotaxis measurement that is done in the convection-free gradient generator $(\mathrm{CF}-\mu \mathrm{Chip})$, and the essential content is about measuring the correct values of chemotaxis parameters: chemotactic sensitivity coefficient $\left(\chi_{0}\right)$ and chemotactic receptor constant $\left(K_{c}\right)$.

Section 4.3 summarizes all the experimental setup for this study, including the test on toluene absorbance in toluene, the device operating procedure and the fitting setup. Section 4.4 discusses all the main results from the CF- $\mu$ Chip. Fluorescein diffusion is studied on the time-lapse, and bacterial chemotaxis is quantitatively measured in presence of different attractant concentrations. Two different bacterial strains are tested and their corresponding chemotaxis parameters are calculated based on the results observed in the CF- $\mu$ Chip. Coupling effect between $\chi_{0}$ and $K_{c}$ is introduced and a feasible way is proposed to solve this problem. The results from this study are also compared with 
previous measurement to find a data relationship. Section 4.5 gives out the implication of the study on chemotaxis related topics.

\subsection{Experimental Section}

\subsubsection{PDMS Test with Toluene}

Because PDMS is hydrophobic, and may absorb organic chemicals like toluene, a simple experiment was designed to test the absorbance in terms of the PDMS swelling ratio. $2 \mathrm{mM}$ toluene solution in $10 \% \mathrm{RMB}$ was prepared and then poured into a $10 \mathrm{~mL}$ amber glass bottle. $2 \mathrm{mM}$ of toluene was selected as the test concentration because this was the largest concentration studied in the PDMS-based CF- $\mu$ Chip. Prior to exposure to toluene, the dimensions of three PDMS blocks were measured by a caliper, and the total weight was also recorded. The PDMS blocks were then put into the $2 \mathrm{mM}$ toluenedissolved buffer and kept in the solution for three hours, and the total volume of the solution and the PDMS blocks depleted the gas phase so that there was no space for toluene to evaporate within the container. The glass bottle was sealed with screwed cap to prevent toluene from evaporating to the surrounding environment. Three hours was the longest time period to carry out toluene related experiments in the PDMS-based CF$\mu$ Chip. After three hours, PDMS blocks were taken out and the dimensions were measured again. It was found that all dimensions had an average $0.1 \%$ increase, and the total weight did not differ significantly from the original value (less than $0.0001 \mathrm{~g}$ increase from $0.6592 \mathrm{~g}$ ). These results demonstrated that no significant absorbance of toluene happened to PDMS blocks during the three-hour experiment. We attributed this to the low concentration of toluene dissolved in water phase. Given these results we 
decided that could be used as an adequate experimental material when $2 \mathrm{mM}$ (or less) toluene solution was studied.

\subsubsection{Experimental Setup}

A time-lapse experiment using fluorescein solution $\left(1.2 \times 10^{-4} \mathrm{M}\right.$ dissolved in $10 \%$ Random Motility Buffer) was done to test the sensitivity and accuracy of the device based on the diffusion theory. Both the fluorescein solution (in light green color) and the buffer were injected into the device at the same flow rate of $1.0 \mathrm{~mL} / \mathrm{h}$, and immediately after the injection, images of the fluorescein diffusion in the cross channels were recorded through the microscope, and the diffusion profiles were plotted according to the distribution of fluorescence intensity.

Then bacterial suspension and attractant solution were injected through the two parallel inlets into the channel by a syringe pump (PHD 2000 Infusion, Harvard Apparatus) at a constant speed of $1.0 \mathrm{~mL} / \mathrm{h}$, which corresponded to $0.38 \mathrm{~mm} / \mathrm{s}$ in the main channel and Reynolds number $\mathrm{Re}=0.28$. At this value of $\mathrm{Re}$, the two streams satisfied the creeping flow criterion and diffusion was the only means of transport in the direction transverse to flow into the opening of the via.

A wide-field epifluorescent microscope (Olympus IX-70) with the $2 \times / 0.05$ (Olympus, Plan) and 20x/0.40 objective (Olympus, PlanFI) was used to observe the bacterial distribution within the channels. A dichroic filter (Chroma $51001 \mathrm{BS}$, double coated for DAPI and TRITC) was used to detect the P. putida's fluorescence, and FITC and DAPI filters (Chroma 31013602 NB) were used for E. coli strains, and FITC was also used for the fluorescein solution. Images about bacterial distribution were taken at 
different locations across the cross channels. Then the bacterial fluorescence was normalized by the following equation:

$$
I_{j}^{n}=\frac{I_{j}-I_{0}}{I_{F}-I_{0}}
$$

I represents the light intensity from bacterial fluorescence and background noise, the superscript $n$ stands for normalized value, subscript $j$ stands for certain location point along the cross channel, $O$ stands for the channel end with no bacterial input, and $F$ stands for the other end with full bacterial density. Because the arc lamp source occasionally generates fluctuating light intensity, each $I$ value was normalized in each image shot by dividing the background intensity from a plain area outside the channel.

\subsubsection{Mathematical Models}

One-dimensional mathematical models were used to evaluate the chemotactic parameters $\chi_{0}$ and $K_{c}$, as diffusion only took place in the transverse direction. The directed migration of the bacterial population due to chemotaxis was represented by the chemotactic velocity, $v_{c h}$, (Chen et al., 1998). The expression of $v_{c h}$ was simplified from the two dimensional Eqn 3.6 to

$$
v_{C h}=\frac{2}{3} v_{b} \tanh \left(\frac{\chi_{0}}{2 v_{b}} \frac{K_{c}}{\left(K_{c}+a\right)^{2}} \frac{\partial a}{\partial x}\right)
$$

As indicated by Eqn 4.2, the chemotactic receptor constant links the concentration of the external chemical stimulus with the chemosensory system.

The chemoattractant gradient in Eqn 4.2 was calculated from the governing equation for the chemoattractant (Eqn 3.3). Because the system was convection free, the convection terms were canceled, then Eqn 3.3 was simplified to have the formulation of Fick's Second Law: 


$$
\frac{\partial c}{\partial t}=D \frac{\partial^{2} c}{\partial x^{2}}
$$

where $D$ is the diffusion coefficient for either bacteria or attractant, and $c$ stands for the concentration for either bacteria or attractant. The boundary conditions for the concentration profile in time and space, $c(x, t)$, are $c(0, t)=c_{B}$ and $c(L, t)=c_{0}$, and the initial condition is $c(x, 0)=0$. The solution has a functional form that depended on the error function:

$$
\frac{c_{0}-c}{c_{0}-c_{B}}=\operatorname{erf}\left(\frac{x}{2 \sqrt{D t}}\right)
$$

where $c_{0}$ is the concentration at the matter's source and $c_{B}$ is the concentration at the end of the diffusion space, and in this case, $c_{B}$ is zero since there is no bacteria/attractant at the other end of channel, so the error function solution could be further simplified. Because the measurement took at the steady state, so the time term on the left hand side of the governing equation (Eqn 3.3) was canceled too. Also, since the diffusion happened in a bulk solution, porosity, $\varepsilon_{p}$, equals to 1 , so $D_{e, a}$ equals to $D_{a}$. Then the onedimensional equation was simplified to

$$
0=D_{a} \frac{\partial^{2} a}{\partial x^{2}}
$$

The ordinary differential equation could be easily solved and $a(x)=a_{0}\left(1-\frac{x}{L}\right)$, where $L$ is the channel length, so concentration profile of attractant should be linear in the channel. Similarly, the governing equation used for bacterial density, $b$, was simplified to

$$
0=\mu_{0} \frac{\partial^{2} b}{\partial x^{2}}-\frac{\partial}{\partial x}\left(v_{C h} b\right)
$$


where $\mu_{0}$ is the bacterial random motility, namely $D_{b}$ in Eqn 3.5, with $b(0)=0$ and $b(L)=$ $b_{0}$.

\subsection{Results and Discussion}

Fluorescein solution was used to determine how well the device worked based on diffusion time in the cross channel. Bacterial distributions of both P. putida and E. coli strains were observed and quantified to measure corresponding chemotaxis parameters. The coupling effect between chemotactic sensitivity coefficient $\chi_{0}$ and chemotactic receptor constant $K_{c}$ was studied and analyzed based on mathematical modeling tools. Generally speaking, errors in fitted values of $\chi_{0}$ and $K_{c}$ may compensate for each other. This was an essential factor that was omitted by many previous quantitative researches on bacterial chemotaxis, and this study introduced a straightforward method to solve the problem created by coupling effect.

\subsubsection{Fluorescein Test}

As shown in Figure 4.1, both the fluorescein solution (in light green color) and the buffer were injected into the device at the same flow rate of $1.0 \mathrm{~mL} / \mathrm{h}$, and a distinct interface formed between the two streams in the channel. Immediately after the injection, images of the fluorescein diffusion in the cross channels were taken by the microscope at different time points (Figure 4.2), and the diffusion profiles were plotted according to the distribution of fluorescent intensity in an hour observation (Figure 4.3). 


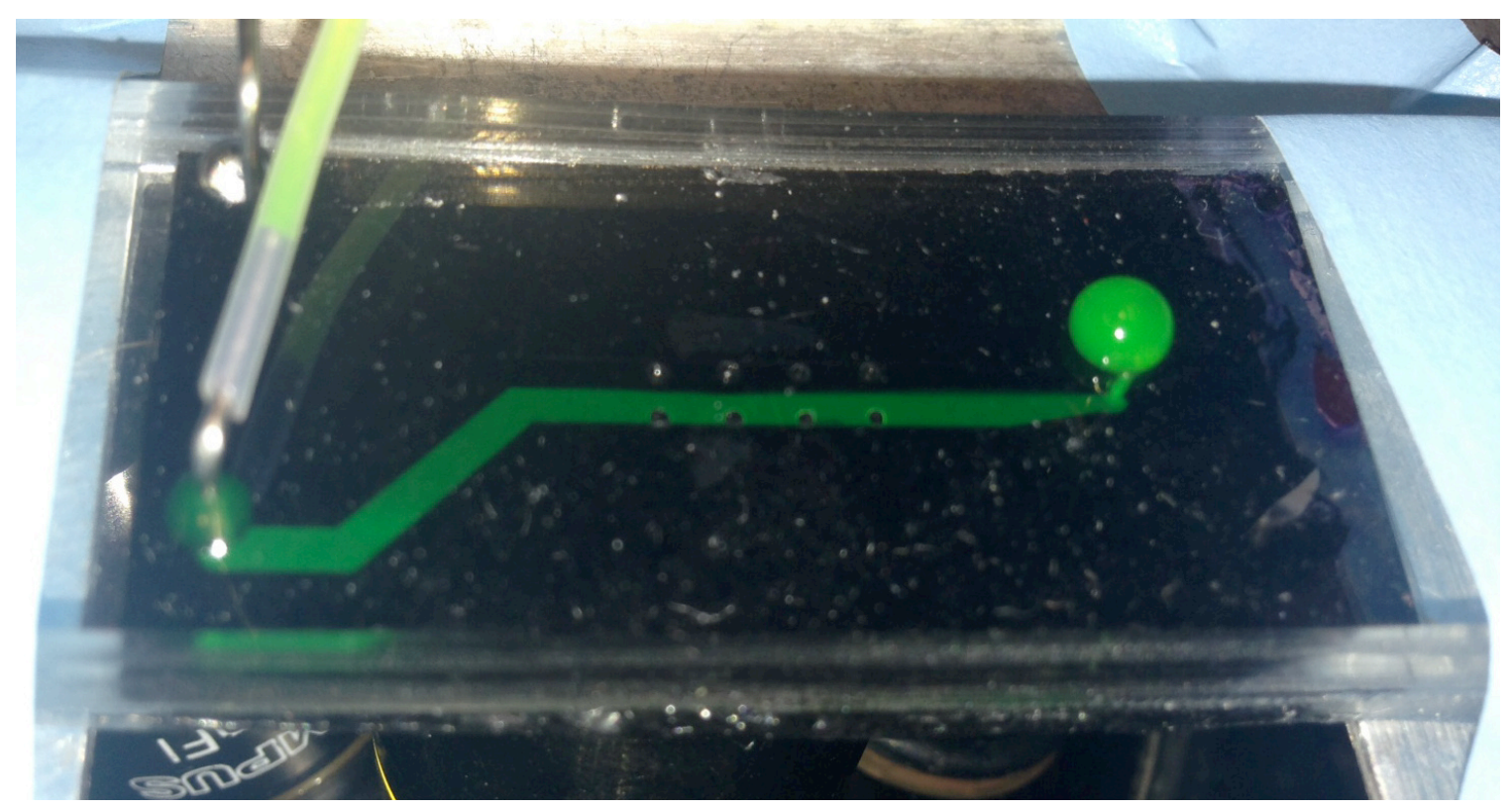

Figure 4.1 Illustration of the injection of both the fluorescein solution (light green color) and the $10 \%$ Random Motility Buffer at the same flow rate.

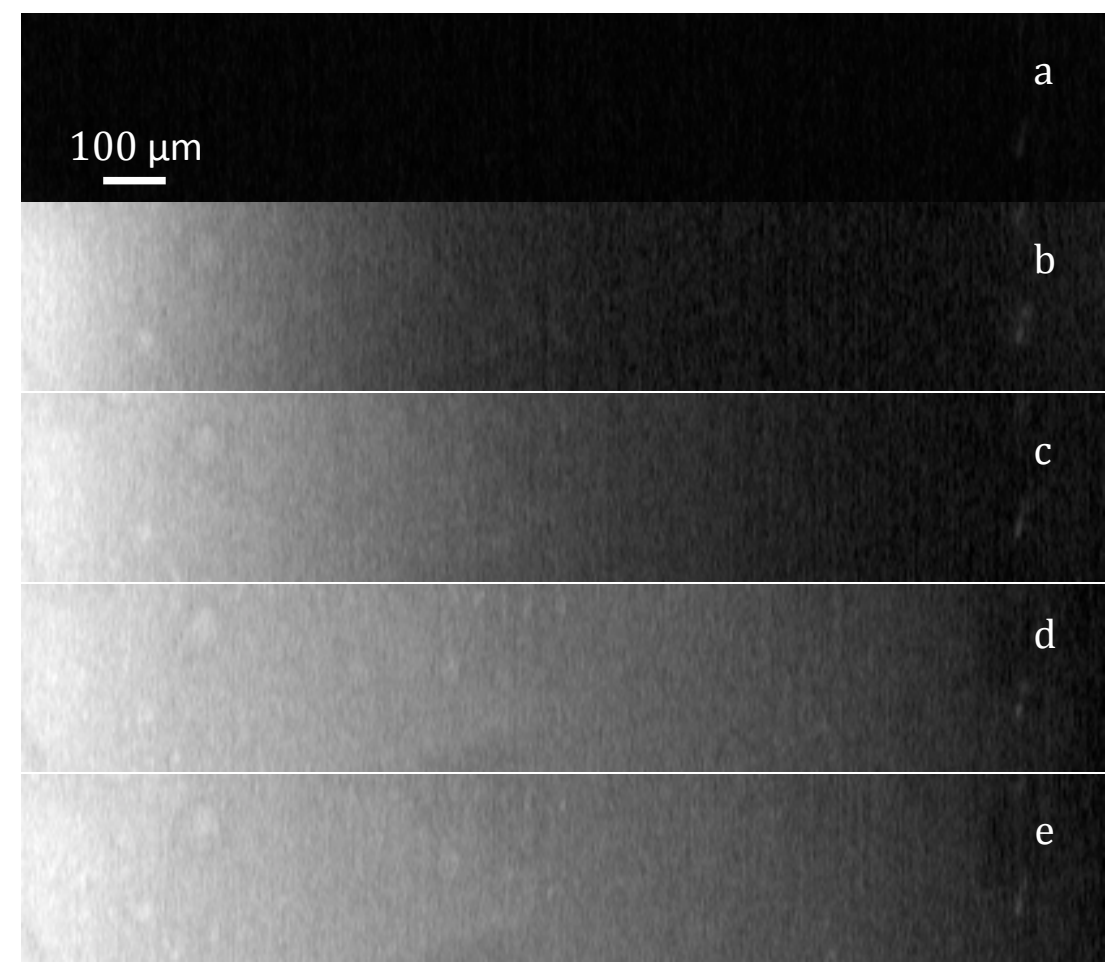

Figure 4.2 Time-lapse distribution of fluorescein solution in the cross channel at times of (a) 0 
min, (b) $3 \mathrm{~min}$, (c) $10 \mathrm{~min}$, (d) $40 \mathrm{~min}$ and (e) $60 \mathrm{~min}$.

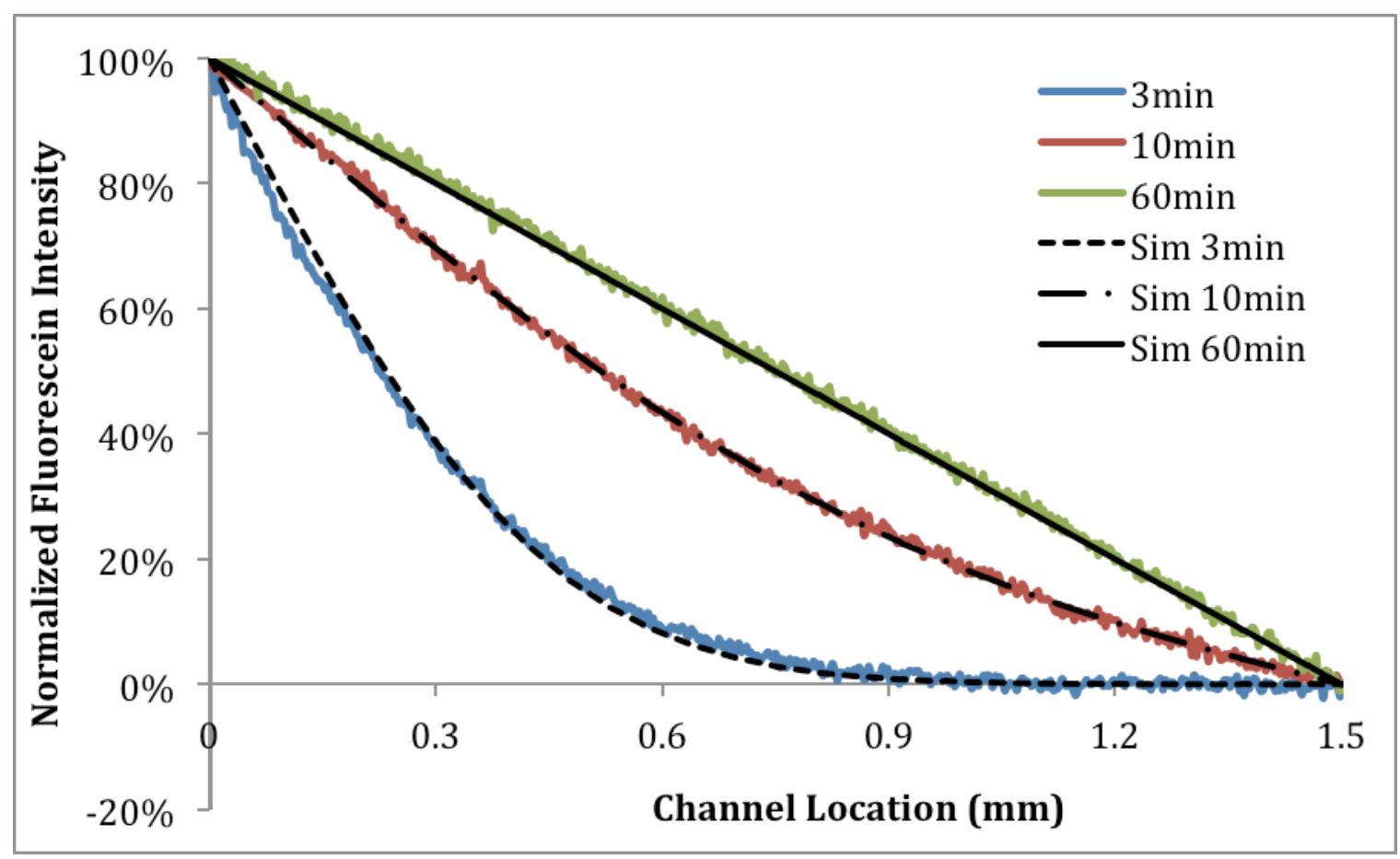

Figure 4.3 Normalized fluorescence of fluorescein within the cross channel at 3,10 and 60 min.

The black curves are the calculated distributions according to the error function solution from Eqn 3.5 using a published value for fluorescein diffusivity of $0.49 \times 10^{-9} \mathrm{~m}^{2} / \mathrm{s}$ (Rani et al., 2005). The coefficient of determination $\mathrm{R}^{2}$ is 0.99 .

As shown in Figure 4.3, the concentration profile of fluorescein reached steady state within 60 minutes from the time of injection, and formed a linear distribution. Fitting curves were superimposed based on the error function solutions from Eqn 3.5 and matched the experimental data well using a diffusion coefficient of fluorescein at $0.49 \times$ $10^{-9} \mathrm{~m}^{2} / \mathrm{s}$, which was reported by Rani et al. (2005). The Einstein relation $t=\frac{L^{2}}{2 D}$ was used to estimate the time to reach steady state, where $L$ was $1.5 \mathrm{~mm}$ and $D$ was $0.49 \times 10^{-}$ 
${ }^{9} \mathrm{~m}^{2} / \mathrm{s}$. The calculation indicates that a time $t$ of 40 minutes is sufficient for the fluorescein distribution to reach steady state in the channel, which is consistent with the results from the model equations.

\subsubsection{Measurement of Bacterial Chemotaxis}

The precise measurement and prediction of fluorescein distribution demonstrated the feasibility to observe bacterial distribution in the CF- $\mu$ Chip. Distributions of chemotactic bacteria in the presence of a linear attractant gradient were then studied in this microfluidic device. Table 4.1 summarizes the time to reach steady state for different bacterial strains and attractants as calculated from the Einstein equation. All bacteria and chemoattractant chemicals achieved steady state within an hour of injection, except for $E$. coli $\mathrm{HCB} 1$, which required 1.3 hours. Based on these predicted results, the observation start point was chosen to be two hours after the injection for P. putida strains and E. coli $\mathrm{HCB} 437$, and four hours after the injection for E. coli $\mathrm{HCB} 1$, in order to ensure the establishment of a steady state system. Figure 4.4 shows an image of $E$. coli HCB1 cells within a single cross channel of the CF- $\mu$ Chip at several times. Note that the diffusion profile did not appear to change very much between two and four hours.

Table 4.1 Calculated time to reach steady state for all bacterial strains and chemicals used in the CF- $\mu$ Chip

$$
\begin{array}{cc}
\text { Random Motility }\left(\mu_{0}\right) \text { or Diffusion } & \text { Time to Reach Steady } \\
\text { Coefficient }\left(D_{a}\right)\left[\times 10^{-10} \mathrm{~m}^{2} / \mathrm{s}\right] & \text { State }[\text { hour }]
\end{array}
$$




\begin{tabular}{lcc}
\hline P. putida $\mathrm{F} 1$ & 13 & 0.24 \\
$P$. putida $\mathrm{F} 1 \mathrm{CheA}$ & 13 & 0.24 \\
E. coli $\mathrm{HCB} 1$ & 2.4 & 1.30 \\
E. coli $\mathrm{HCB} 437$ & 17 & 0.18 \\
Toluene & 9.5 & 0.33 \\
$\alpha$-methylaspartate & 8.6 & 0.36 \\
\hline
\end{tabular}

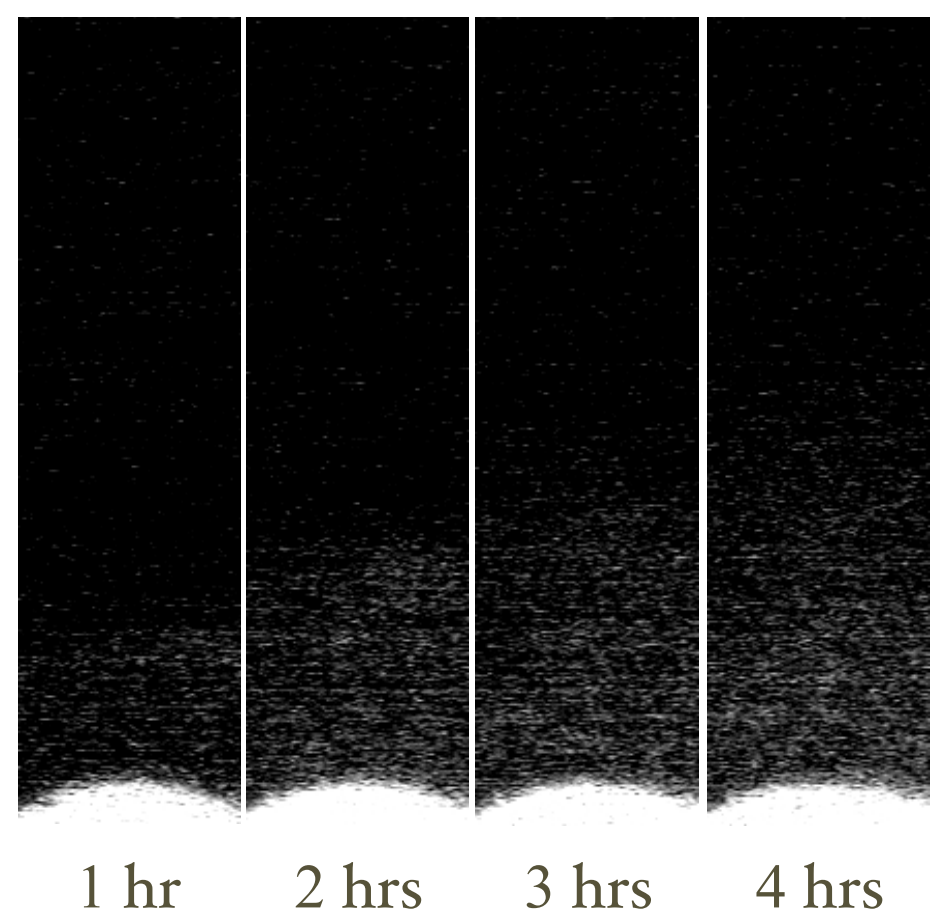

Figure 4.4 Bacterial distribution (E. coli $\mathrm{HCB} 1)$ within a cross channel at different times. The gray level intensity in the image corresponds to the bacterial density.

Bacterial suspension and attractant solution were then injected into inlets on opposite sides and formed two parallel streams. The P. putida distribution in the presence 
of toluene at steady state is shown in Figure 4.5 and 4.6. In the absence of a chemotactic response, the governing equation for bacterial distribution at steady state has the same form as that for an attractant (Eqn 4.3), which means that the bacterial density also follows a linear distribution with a constant gradient in the cross channel, as shown by both the experimental data (red points) and the fitting result (red line) of the nonchemotactic mutant ( $P$. putida F1 CheA) in Figure 4.6. For chemotactic bacteria $P$. putida $\mathrm{F} 1$ in the presence of a concentration gradient of attractant, the advective term induced by chemotaxis in the governing equation (Eqn 4.4) caused more bacteria to migrate into the cross channel from the parallel flow channel. As a result, the bacterial distribution deviated positively from the linear distribution and formed a parabolic shape, as shown by the blue and orange curves in Figure 4.6. The extent of deviation was determined by the strength of the chemotactic response as well as the attractant concentration of the local environment. In Figure 4.6, the data points in both blue and orange groups were collected from the chemotactic $P$. putida F1. The difference was a different attractant concentration introduced at the opposite end of the cross channel: the toluene concentration used for the blue diamonds was $2 \mathrm{mM}$, and that for the orange triangles was $0.2 \mathrm{mM}$. A two-way ANOVA test showed that the difference was statistically significant between the two chemotactic experiments $(2$ and $0.2 \mathrm{mM})$ with $\mathrm{p}$ $<0.0001$, the difference was also significant between the $2 \mathrm{mM}$ and nonchemotactic control with $\mathrm{p}<0.0001$, but the difference between the $0.2 \mathrm{mM}$ and the control was not statistically significant. 


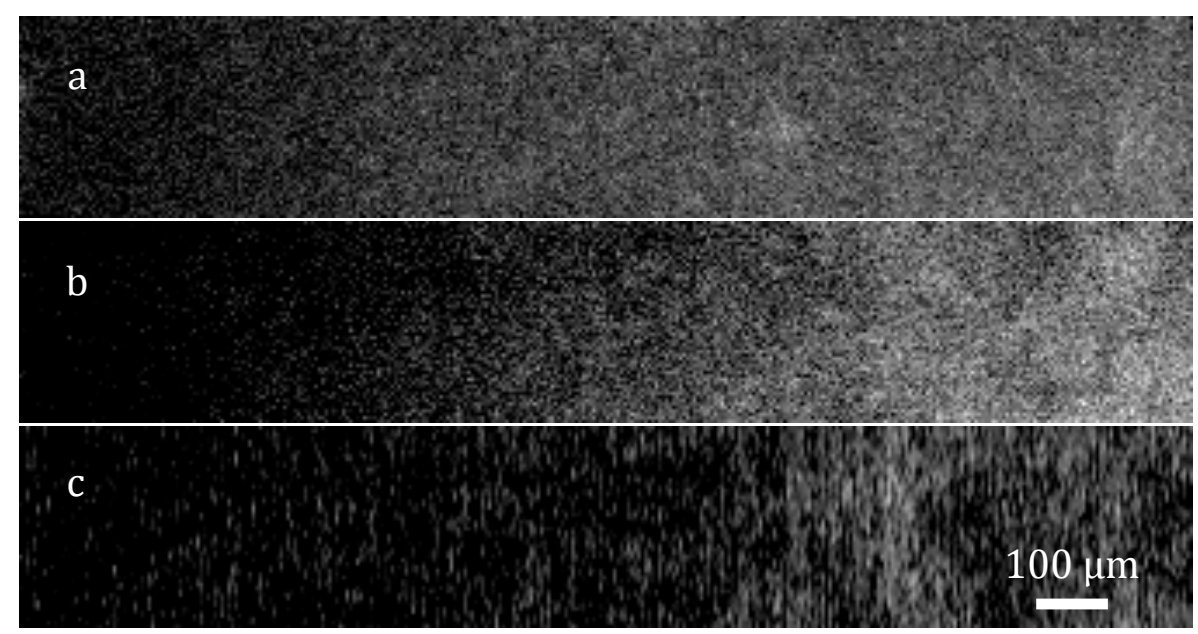

Figure 4.5 The images of $P$. putida distribution in a cross channel at steady state. The gray level intensity represents the bacterial density. The distribution of $P$. putida $\mathrm{F} 1$ is observed in presence of (a) $2 \mathrm{mM}$ toluene as the attractant at the opposite side, and (b) $0.2 \mathrm{mM}$ toluene, and (c) the distribution of $P$. putida $\mathrm{F} 1 \mathrm{CheA}$ is also recorded in presence of $2 \mathrm{mM}$ toluene. 


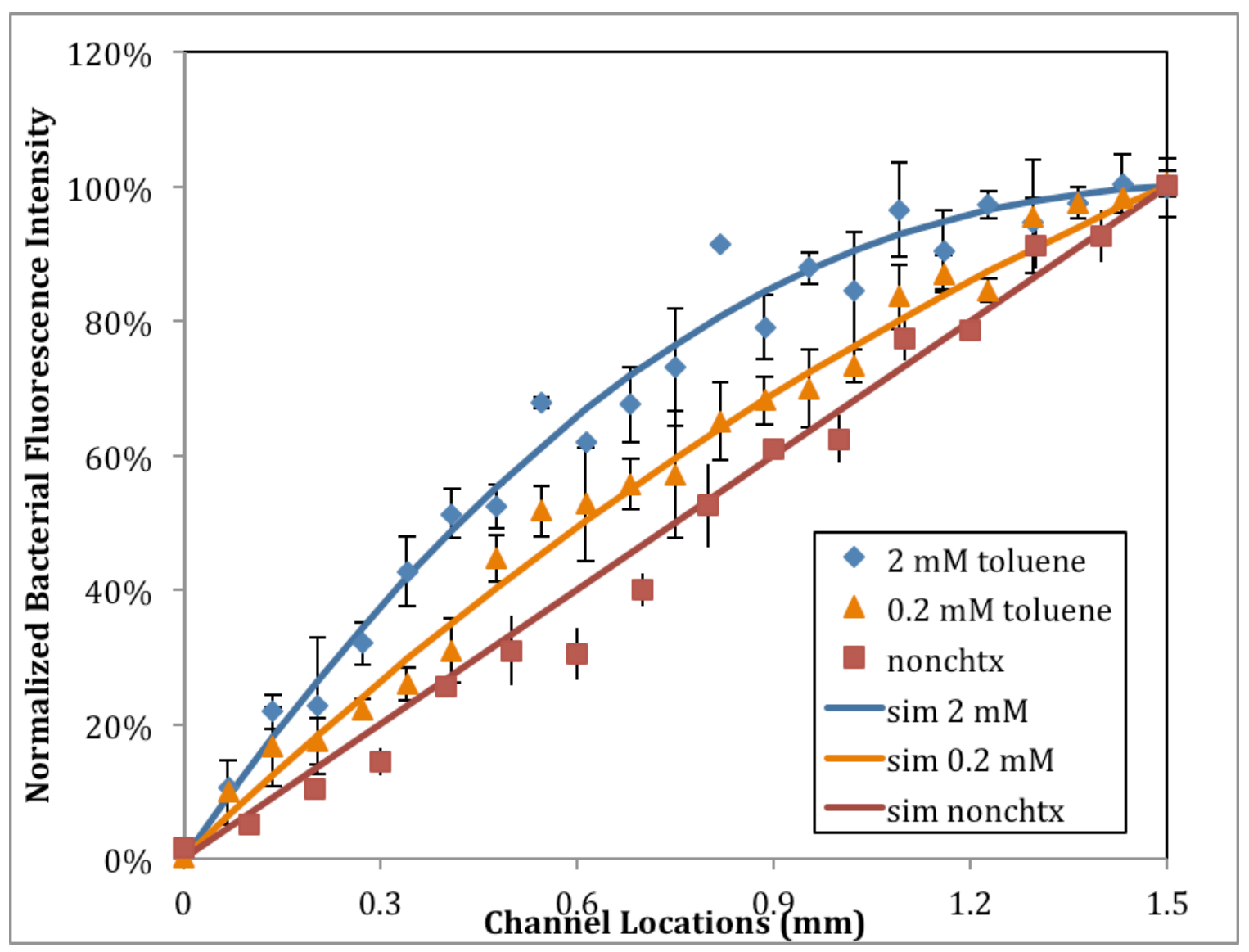

Figure 4.6 P. putida distribution in the cross channel of CF- $\mu$ Chip at steady state. Attractant diffuses from left to right in the figure, and its concentration is $2 \mathrm{mM}$ for the blue diamond data and $0.2 \mathrm{mM}$ for the orange triangles. Experimental results of bacterial density are plotted as dots (nonchemotaxis in red), and the corresponding lines are the fitting results.

The rationale for choosing these two particular concentrations will be discussed in the following section about decoupling the effect of the chemotaxis parameters. In some previous work (Olson et al., 2004; Lanning et al., 2008; Law and Aitken, 2005; Wang et al., 2012) just one attractant concentration was tested, and because the chemotactic sensitivity coefficient, $\chi_{0}$, and chemotactic receptor constant, $K_{c}$, could compensate for each other in the expression of chemotactic velocity (Eqn 4.2), it was necessary to 
estimate a value for one of the parameters (usually $K_{c}$ ) in order to fit the other. In this study experiments were conducted with two different attractant concentrations. Ford and Lauffenburger (1991) checked the relationship between $\chi_{0}$ and a series of attractant concentrations using stopped-flow diffusion chamber (SFDC), and found that $\chi_{0}$ was independent on the attractant concentration. The work in the dissertation was a follow up of this idea using a different experimental system. The new experimental system (CF$\mu$ Chip) allowed to observing bacterial distribution at steady state, while SFDC detected the bacterial density as a function of time, so CF- $\mu$ Chip eliminated the possible effect of time during measurement. Experiments with the $2 \mathrm{mM}$ toluene source attracted more chemotactic bacteria into the cross channel than the $0.2 \mathrm{mM}$ one, as the area under the distribution curve in blue was greater than the one in orange. This result also indicated that the correct $K_{c}$ for $P$. putida/toluene was the same order of magnitude as $2 \mathrm{mM}$. Furthermore, the value of $K_{c}$ was within the range of previously reported values for similar chemical contaminants, 0.001 to $1 \mathrm{mM}$ (Parales et al., 2000).

The qualitative experimental results were further supported by the quantitative comparison to model equations at both attractant concentrations. Fitting curves indicated that only a $K_{c}$ value near $1 \mathrm{mM}$ could match the experimental results at both toluene concentrations in Figure 4.6. The fitting results indicate that the chemotactic sensitivity coefficient $\chi_{0}$ is $1.8 \pm 0.7 \times 10^{-4} \mathrm{~cm}^{2} / \mathrm{s}$, and chemotactic receptor constant $K_{c}$ is $1 \pm 0.4 \mathrm{mM}$. The value of $\chi_{0}$ is in the same magnitude as the average value for P. putida F1 to TCE $\left(5.4 \times 10^{-4} \mathrm{~cm}^{2} / \mathrm{s}\right)$, and $K_{c}$ is also same as the reported value for $P$. putida $\mathrm{F} 1 / \mathrm{TCE}$ at 1 $\mathrm{mM}$ (Olson et al., 2004). Moreover, because $K_{c}$ represents the optimal concentration for the chemotactic bacteria to accumulate, the greatest difference between chemotactic and 
nonchemotactic bacterial accumulations when toluene concentration is $2 \mathrm{mM}$ occcurs at the midpoint of the graph, where the toluene concentration is close to $1 \mathrm{mM}$. Figures 4.7 and 4.8 show results similar to Figures 4.5 and 4.6, but using a different bacterial strain. The chemotactic wild type is E. coli $\mathrm{HCB} 1$ and the nonchemotactic control is HCB437 (smooth-swimming mutant), and the chemoattractant is $\alpha$-methylaspartate. Modeling fits to experimental data show that $\chi_{0}$ is $2.4 \pm 0.5 \times 10^{-4} \mathrm{~cm}^{2} / \mathrm{s}$ and $K_{c}$ is $0.13 \pm 0.03 \mathrm{mM}$, both supported by previous work (Lewus and Ford, 2001). One interesting fact is that $K_{c}$ for $E$. coli is almost an order of magnitude lower than P. putida, and the largest differences between chemotactic and nonchemotactic bacteria occurs when the initial attractant concentration is approximately equal to the value of $K_{c}$.

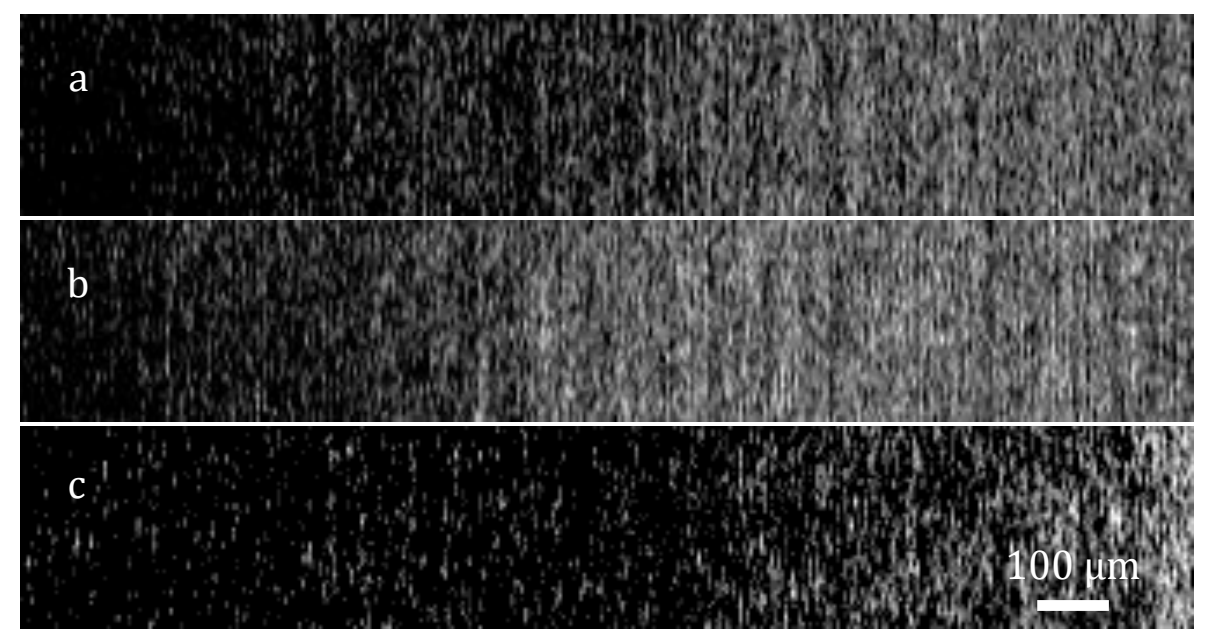

Figure 4.7 The images of E. coli distribution in a cross channel at steady state. The gray level intensity represents the bacterial density. The distribution of E. coli $\mathrm{HCB} 1$ is observed in presence of (a) $3 \mathrm{mM} \alpha$-methylaspartate as the attractant at the opposite side, and (b) $0.3 \mathrm{mM} \alpha-$ methylaspartate, and (c) the distribution of nonchemotactic E. coli HCB437 is also recorded in presence of $3 \mathrm{mM} \alpha$-methylaspartate. 


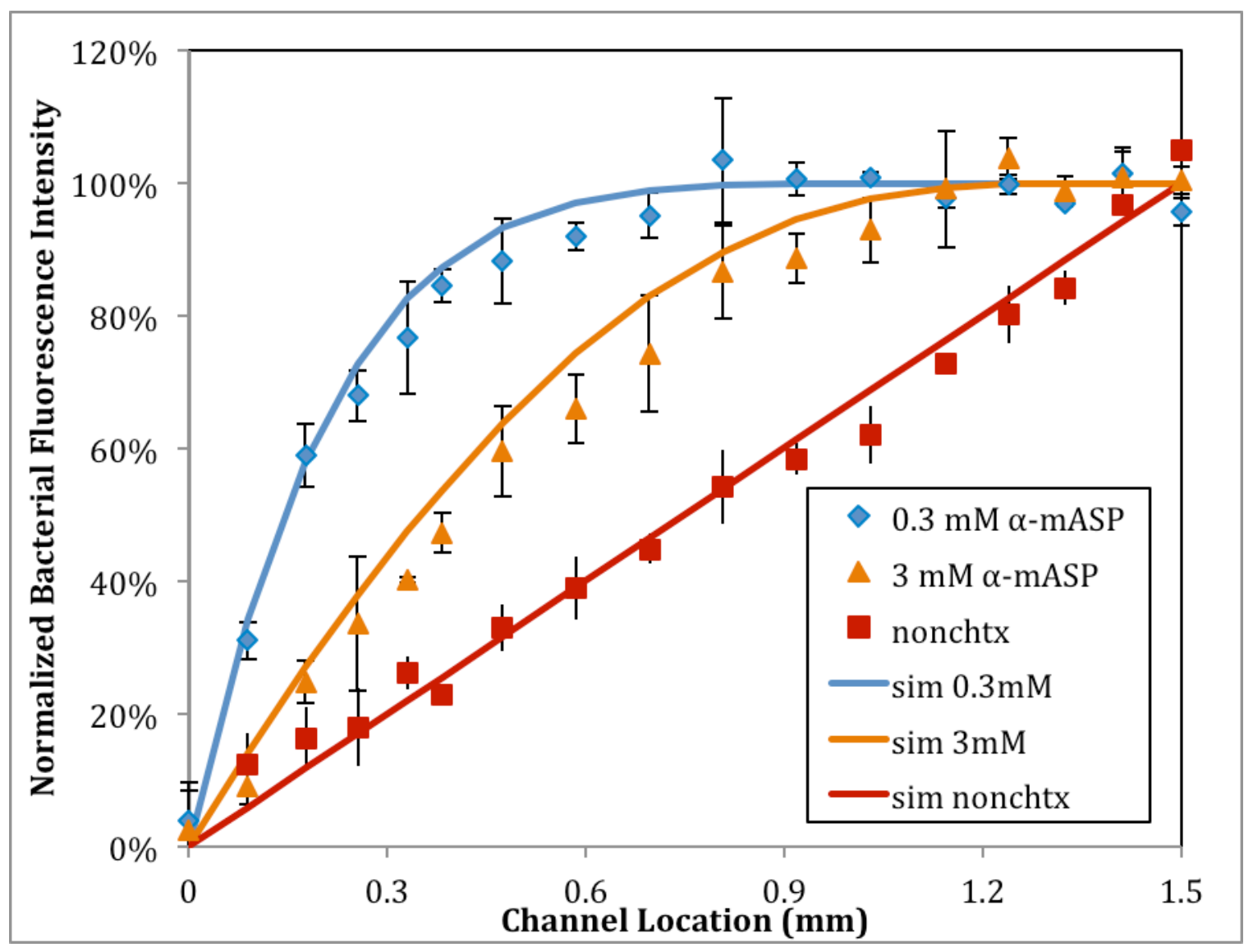

Figure 4.8 E. coli distribution in the diffusion-only channel at steady state. Attractant diffuses from left to right in the figure, and its concentration at the left-hand side is $0.3 \mathrm{mM}$ for the blue diamond data and $3 \mathrm{mM}$ for the orange triangles. Experimental results of bacterial density are plotted as symbols (nonchemotaxis in red), and the corresponding lines are the fitting results.

\subsubsection{Independent evaluations of $\chi_{\underline{0}}$ and $K_{\underline{c}}$}

One essential problem that was omitted by previous studies was the coupling effect between $\chi_{0}$ and $K_{c}$. Ford and Lauffenburger investigated the values of $\chi_{0}$ over a range of initial attractant concentrations in SFDC, and found $\chi_{0}$ was independent on the change of the attractant concentration. The effect of $K_{c}$ on bacterial distribution was still not explicitly studied in presence of different attractant concentrations, and we found that 
this new topic about $K_{c}$ was important to inspect in order to obtain accurate values of $\chi_{0}$ and $K_{c}$. Figure 4.9 illustrates results for different combinations of $\chi_{0}$ and $K_{c}$ values that yield similar curves for bacterial distribution in the cross channel. Table 4.2 lists the wide range of values for the chemotaxis parameters used to generate the simulation curves in Figure 4.9.

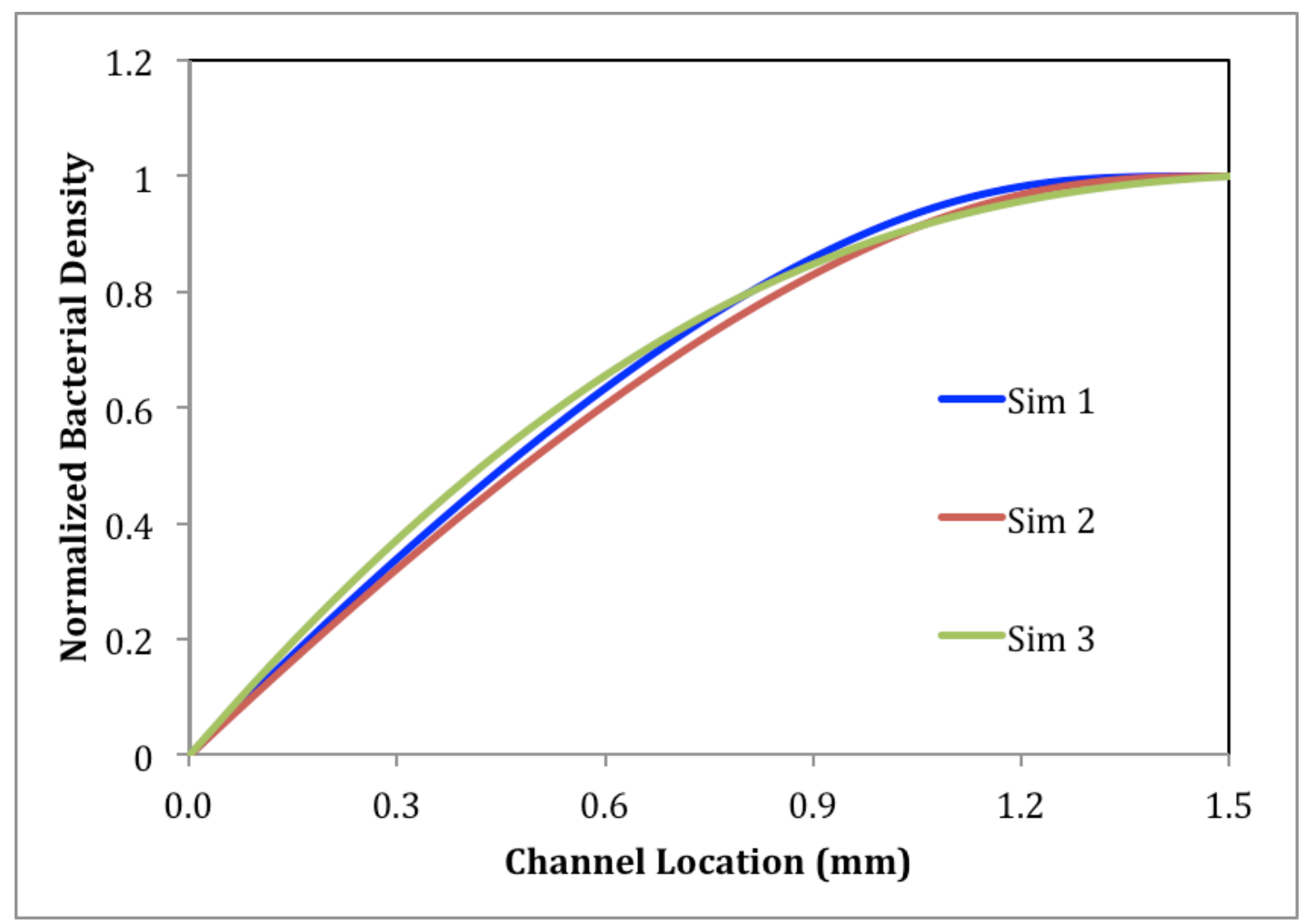

Figure 4.9 Similar simulation curves resulted from the combinations of different $\chi_{0}$ and $K_{c}$ values.

Table 4.2 $\chi_{0}$ and $K_{c}$ values used for the different simulation curves in Figure 4.9.

\begin{tabular}{lcc}
\hline & $\chi_{0}\left[\times 10^{-8} \mathrm{~m}^{2} / \mathrm{s}\right]$ & $K_{c}[\mathrm{mM}]$ \\
\hline $\operatorname{Sim} 1$ & 36 & 0.01 \\
\hline
\end{tabular}




\begin{tabular}{lcc}
\hline $\operatorname{Sim} 2$ & 3.6 & 0.1 \\
$\operatorname{Sim} 3$ & 1.8 & 1 \\
\hline
\end{tabular}

As shown in Table 4.2, even as the value of $K_{c}$ was increased from 0.01 to $1 \mathrm{mM}$, the simulation curves resulted in nearly the same bacterial distribution by an appropriate decrease in $\chi_{0}$. Many of the previous studies related to chemotaxis (Olson et al., 2004; Lanning et al., 2008; Law and Aitken, 2005; Wang et al., 2012) assumed a value for $K_{c}$ based on published values and then determined $\chi_{0}$ by a curve-fitting exercise. However, the reported $K_{c}$ values were specific to certain bacteria/ attractant pairs; in order to apply appropriate values of $\chi_{0}$ and $K_{c}$ to a different bacteria/attractant pair, a direct measurement of $K_{c}$ for the pair of interest was needed. The effect that each parameter has on the bacterial distribution when varied independently of the other is illustrated in Figures 4.10 and 4.11. The default value for $\chi_{0}$ was set as $1 \times 10^{-8} \mathrm{~m}^{2} / \mathrm{s}$ and $K_{c}$ as $0.1 \mathrm{mM}$ for both figures.

Figures 4.10 (a) and (b) show the normal changes of $\chi_{0}$ and $K_{c}$ in the cross channel with the attractant concentration of $2 \mathrm{mM}$. In other words, the increase in either $\chi_{0}$ or $K_{c}$ could increase the bacterial population in the channel, which explained why the fitting curves overlapped each other over a wide value range of $\chi_{0}$ and $K_{c}$. 

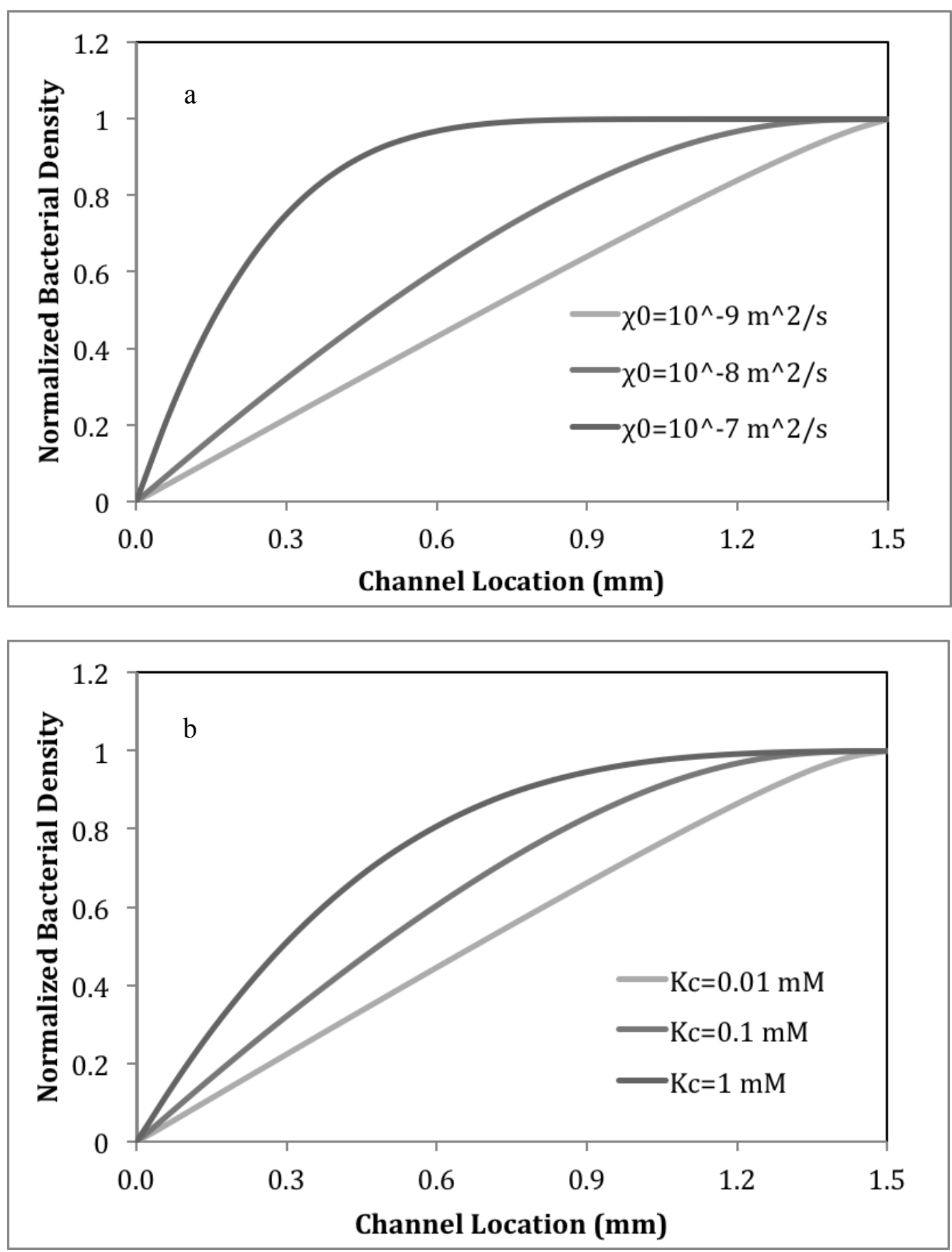

Figure 4.10 Simulated results of the impact of changing $\chi_{0}$ and $K_{c}$ on bacterial distribution in presence of $2 \mathrm{mM}$ of chemoattractant. The default value for $\chi_{0}$ was set as $1 \times 10^{-8} \mathrm{~m}^{2} / \mathrm{s}$ and for $K_{c}$ as $0.1 \mathrm{mM}$, and (a) shows the impact of $\chi_{0}$ with a fixed $K_{c}$ at the default value, and (b) shows the impact of $K_{c}$ with a fixed $\chi_{0}$ at the default value. 
By performing the experiment or simulation at two different attractant concentrations, it was possible to evaluate both parameters, independently. Figure 4.11 shows results for the simulation in the Figure 4.10 at a lower attractant concentration of $0.2 \mathrm{mM}$. While the $\chi_{0}$ value still showed a positive influence on bacterial population in the channel, the impact of $K_{c}$ did not follow the same trend as the results from the higher level of attractant concentration. As shown by Figure 4.11 (b), the middle $K_{c}$ value of 0.1 $\mathrm{mM}$ resulted in the most bacterial population in the channel, and $1 \mathrm{mM}$ resulted in the least. This was because that the attractant concentration of $0.2 \mathrm{mM}$ covered the optimal locations for chemotactic bacteria with $K_{c}$ of $0.1 \mathrm{mM}$ in its concentration gradient; however, bacteria with $K_{c}$ of $1 \mathrm{mM}$ could not find their optimal location in this concentration gradient started from $0.2 \mathrm{mM}$. In other words, the correct $K_{c}$ could be determined first by tailoring the concentration gradient of attractant to a certain range, and $\chi_{0}$ could then be fitted using the specific $K_{c}$. Therefore, the coupling effect between the two chemotaxis parameters could be solved. 

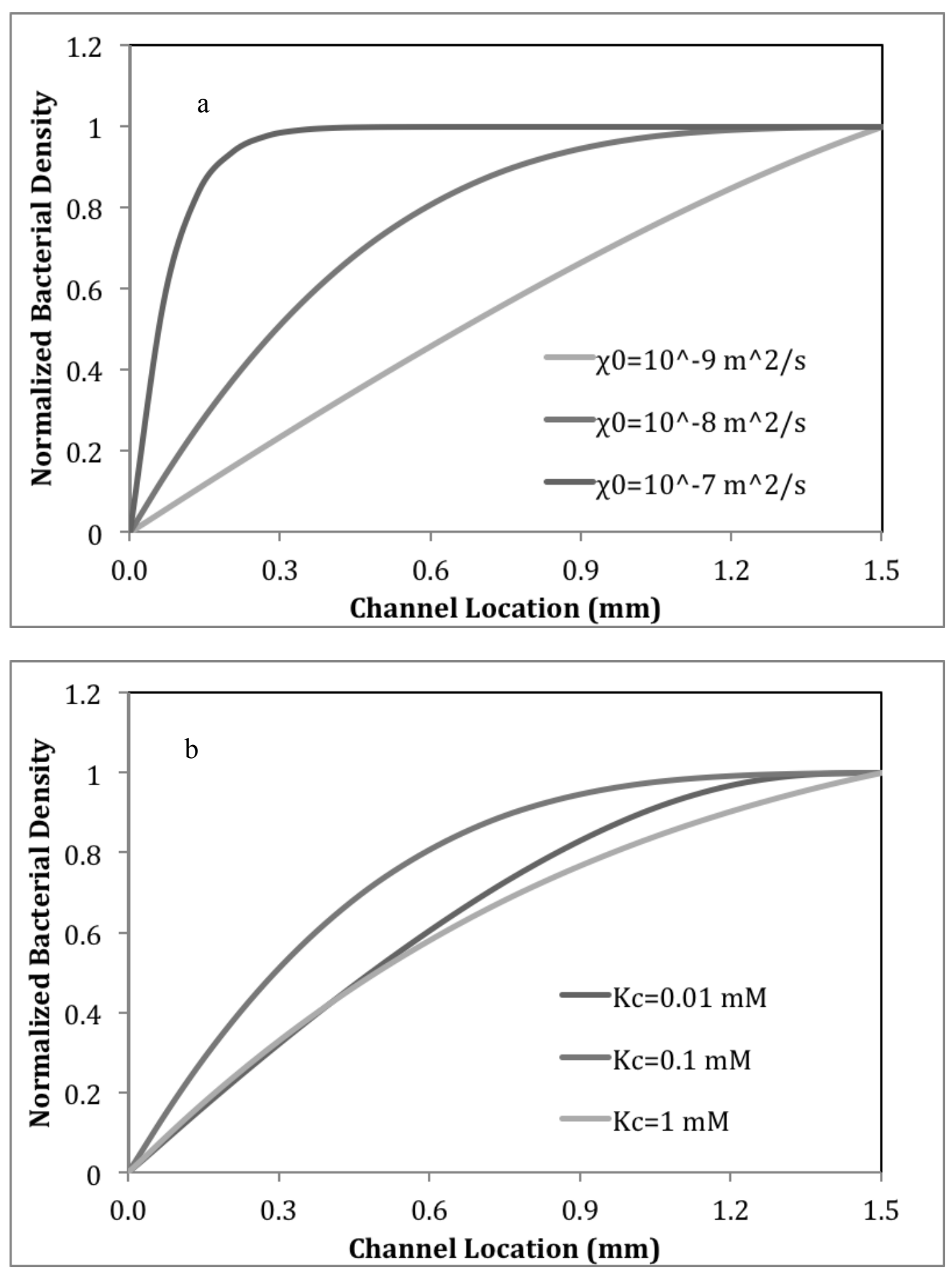

Figure 4.11 Simulated results about the impact of changing $\chi_{0}$ and $K_{c}$ on bacterial distribution in presence of $0.2 \mathrm{mM}$ of chemoattractant. The default values for $\chi_{0}$ is set as $1 \times 10^{-8} \mathrm{~m}^{2} / \mathrm{s}$ and $K_{c}$ as $0.1 \mathrm{mM}$, and (a) shows the impact of $\chi_{0}$ with a fixed $K_{c}$ at the default value, and (b) shows the impact of $K_{c}$ with a fixed $\chi_{0}$ at the default value. 


\subsubsection{Comparison with previous $K_{\underline{c}}$ measurement}

Brashear (2009) measured the chemotactic receptor constant, $K_{c}$, of $P$. putida F1 to benzoate using an agarose plug assay. The methodology for this experiment and its data analysis are summarized in the Appendix. Plug assays were prepared following the procedure from Yu and Alam (1997), and the setup is shown in Figure 4.12. The central agarose plug contained a specific attractant concentration and was surrounded by a bacterial suspension containing a $10 \times$ lower attractant concentration. The plug concentrations ranged from $10^{-6}$ to $10^{-1} \mathrm{M}$. Bacterial accumulation in vicinity of the central plug was observed under the microscope and the $K_{c}$ value was determined from the geometric average concentration, which elicited the greatest accumulation of bacteria.

a

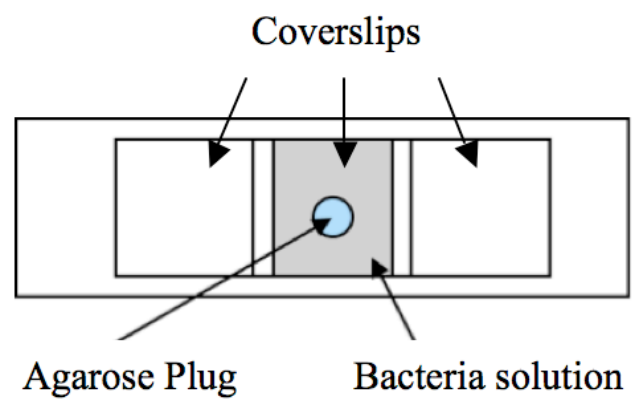

b

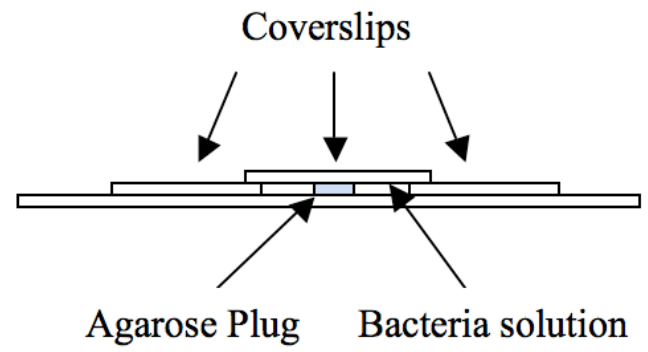

Figure 4.12 Agarose plug assay diagram with (a) top view and (b) side view (Courtesy of Brashear).

Brashear and coworkers (unpublished data) found that P. putida F1 accumulation in presence of benzoate at different concentrations followed a bimodal distribution, as shown in Figure 4.13. The two corresponding peaks in the figure indicated the two 
distinct values of $K_{c}$. The peak in accumulation on the right hand side of the figure as represented by the model curve indicated that $K_{c, h i g h}$ was $0.95 \mathrm{mM}$, and the lower peak in accumulation on the left hand side of the figure indicated that $K_{c, \text { low }}$ was $0.0027 \mathrm{mM}$. The coexistence of $K_{c, h i g h}$ and $K_{c, l o w}$ was supported by experimental findings and mathematical models for two receptor subpopulations (Clarke and Koshland, 1979; Ford and Lauffenbruger, 1991). The two different receptor sites might interact with the same stimulus molecules with different affinity capacities: one showed a low affinity to the attractant at a high external attractant concentration $\left(K_{c, h i g h}\right)$, and the second receptor corresponded to a high affinity at a low attractant concentration $\left(K_{c, \text { low }}\right)$. The attractant concentration corresponding to $K_{c, h i g h}$ was $3 \mathrm{mM}$ in the plug and $0.3 \mathrm{mM}$ in the surrounding bacteria solution, and the attractant concentration corresponding to $K_{c, \text { low }}$ was $0.008 \mathrm{mM}$ in the plug and $0.0008 \mathrm{mM}$ in the surrounding bacteria solution. These values were within the typical range for chemotactic receptor constant, $K_{c}$, from $1 \times 10^{-6} \mathrm{M}$ to 1 $\times 10^{-3} \mathrm{M}$ (Dahlquist et al., 1972; Ford and Lauffenbruger, 1991; Jasuja et al., 1999). 


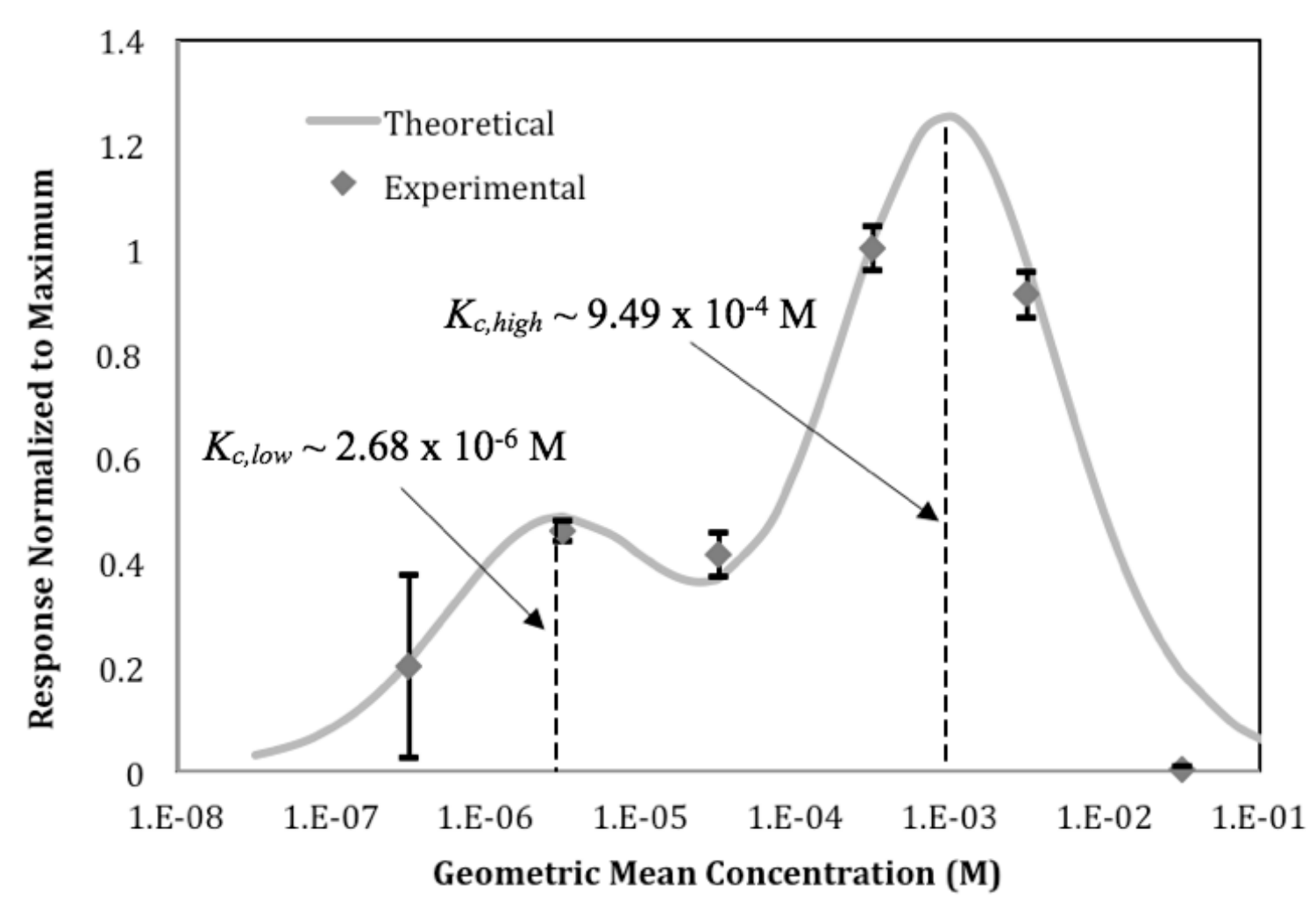

Figure 4.13 Chemotactic response of $P$. putida $\mathrm{F} 1$ to sodium benzoate at different concentrations in agarose plug assay (Brashear, 2009).

The results of $P$. putida $\mathrm{F} 1$ accumulation to toluene were compared with Brashear's work that was discussed above. Toluene and benzoate have many similarities. First of all, they are both simple aromatic components. Secondly, they are both chemoattractants to P. putida F1 (Parales et al., 2000) and are degradable to this bacterial strain. More importantly, evidence shows that the broad-substrate-specificity chemoreceptors may exist in P. putida $\mathrm{F} 1$ to detect a variety of aromatic hydrocarbons, including toluene and benzoate (Parales et al., 2000).

Besides the similarities between toluene and benzoate, the two experimental systems shared some common features. Both the plug assay and CF- $\mu$ Chip were designed to look at the bacterial distribution in presence of a constant attractant source in which a 
predictable concentration gradient was formed. One difference was that the bacterial distribution in $\mathrm{CF}-\mu \mathrm{Chip}$ was measured at steady state, while it was measured at unsteady state in plug assay. Although the measurement was taken at unsteady state in plug assay, the time points to record the images were the same among all the experimental trials, so there was no time variable affecting the bacterial distribution at different attractant concentrations. Another difference was that the plug assay had a 10 times diluted attractant concentration in the surrounding bacterial suspension, but the attractant concentration was zero for the bacterial end in CF- $\mu$ Chip. In order to test if this difference could be neglected, fitting of bacterial distribution was then done by modifying the toluene concentration to $0.2 \mathrm{mM}$ at the bacterial end, while the other end was still $2 \mathrm{mM}$. Figure 4.14 shows the comparison of bacterial distribution between the new and the old assumption on attractant concentration at the bacterial end. The two simulated bacterial distributions had a similar trend, and the biggest difference between them was only approximately $4 \%$, which could be neglected compared to the overall bacterial population. Therefore, the benzoate related data from plug assay and the toluene related data from $\mathrm{CF}-\mu \mathrm{Chip}$ were compared and plotted together in Figure 4.15. The density differences between chemotactic and nonchemotactic bacteria were calculated based on both the experimental and simulation data, and were compared with part of Brashear's data at specific attractant concentrations, with the maximum value in bacterial density normalized to 1 and the geometric mean concentration in Figure 4.13 converted to the attractant concentration in the plug. 

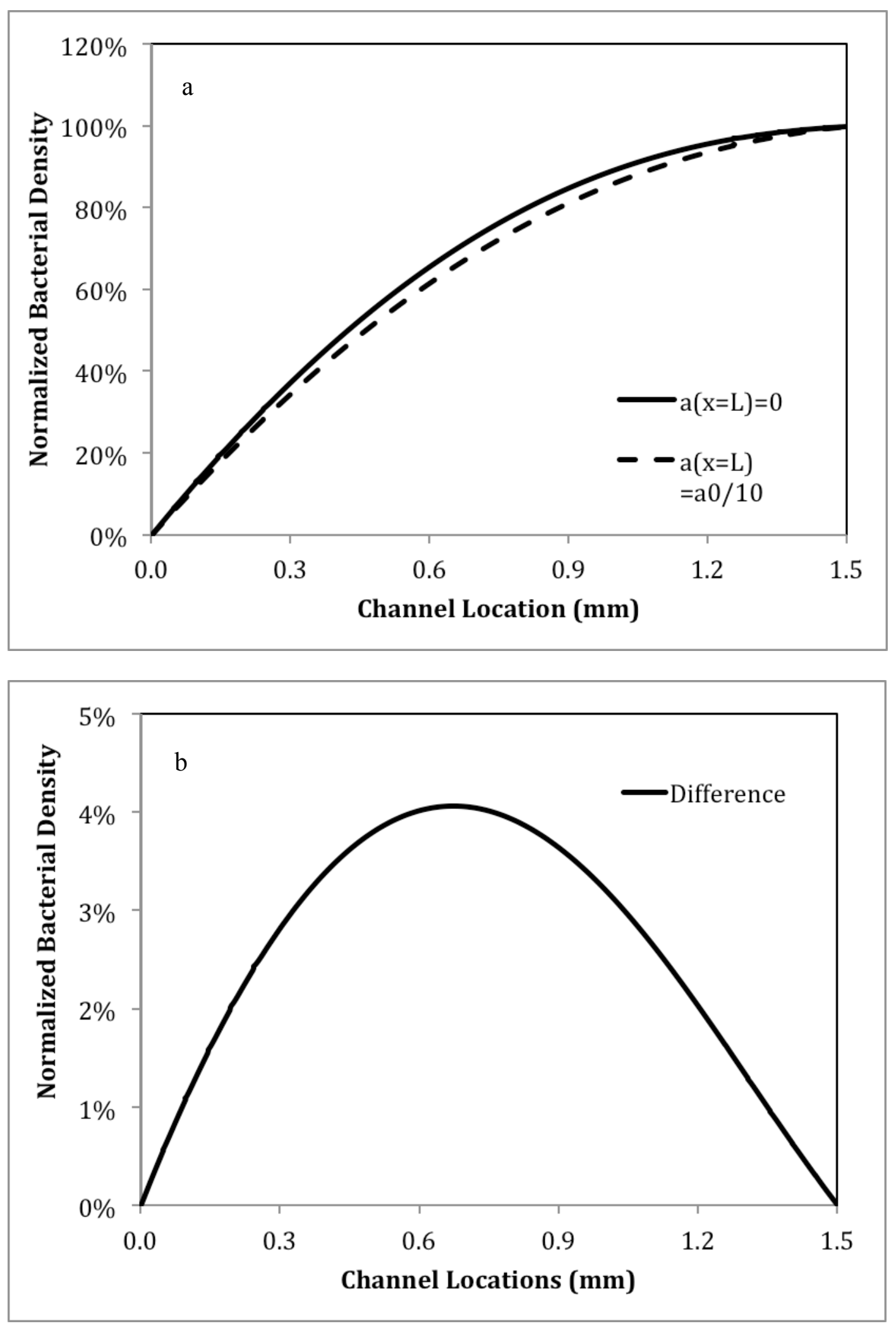

Figure 4.14 (a) Simulation of chemotactic bacterial distribution with different attractant 
concentration at the bacterial end. The solid curve is generated from the zero attractant background, and the dashed curve is from the background with the diluted attractant concentration at the bacterial end. (b) The differences between the two curves in (a) along the channel direction.

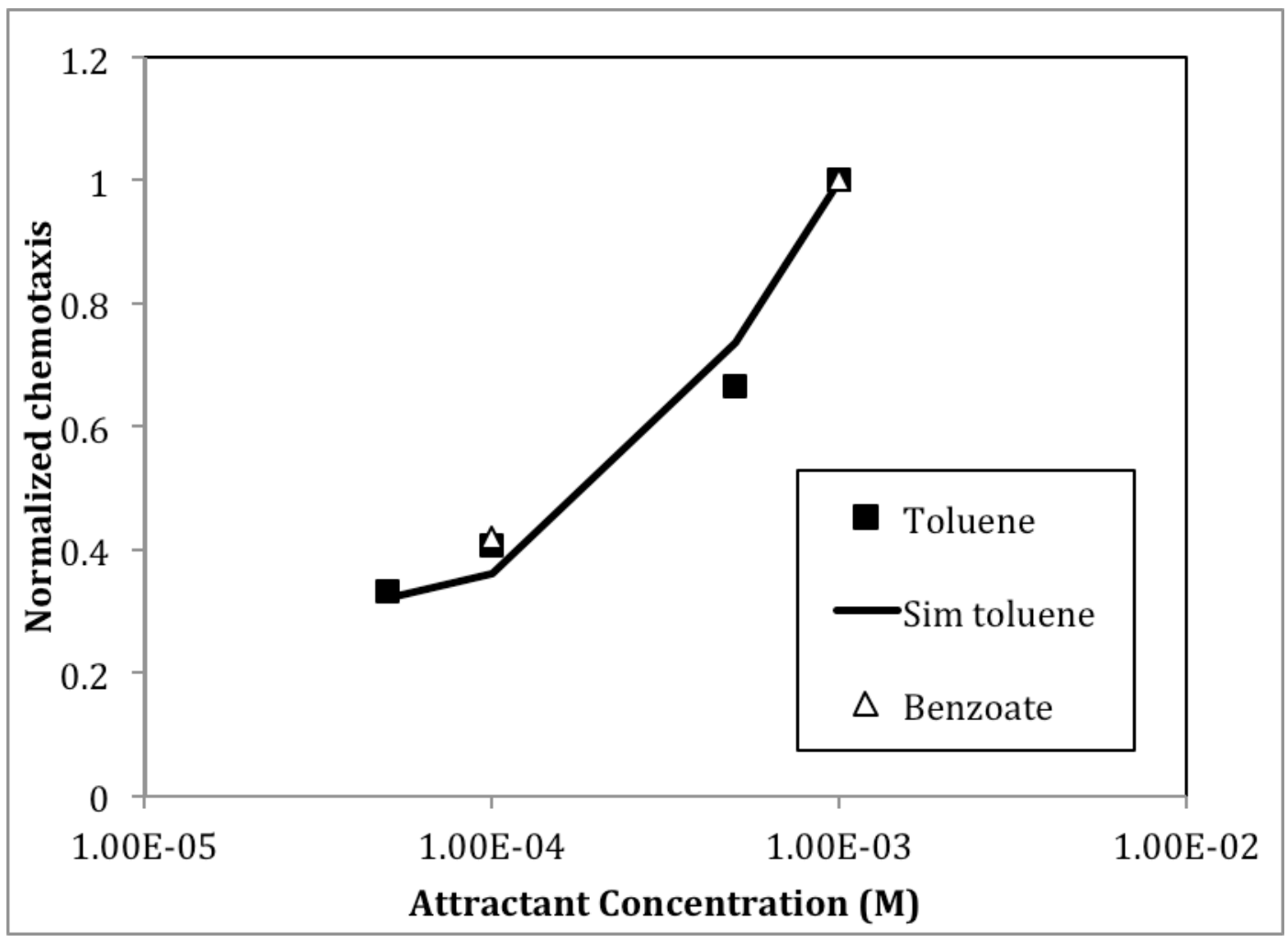

Figure 4.15 Comparison between the benzoate related data (open triangle) from plug assay and the toluene related data (solid square) from $\mathrm{CF}-\mu \mathrm{Chip}$. The black line connects the fitting data for bacterial distribution to toluene.

The toluene related data only covered the range of attractant concentration from 0.05 to $1 \mathrm{mM}$, and benzoate related data points in this range matched exactly with the toluene related trend. In addition, both studies provided a $K_{c}$ value of around $1 \mathrm{mM}$. All these findings indirectly suggest the possibility of the earlier statement: broad-substrate- 
specificity chemoreceptors may exist in P. putida $\mathrm{F} 1$ to detect a variety of aromatic hydrocarbons, including toluene and benzoate. However, this study did not find enough evidence for $K_{c, \text { low }}$ at a lower attractant concentration; the $K_{c, \text { low }}$ might only exist for benzoate, or its induced accumulation was concealed by the limited precision in measurement. More studies targeting the lower concentration ranges can be done to test the possibility of $K_{c, \text { low' }}$ 's existence for P. putida and other bacterial strains. The same experimental procedure is suggested except using a lower initial toluene concentration close to the reported value of $K_{c, l o w}$ at $0.0027 \mathrm{mM}$, and a fitting showed (data not shown) that parabolic bacterial distribution is similar to the one for $P$. putida $\mathrm{F} 1$ at $2 \mathrm{mM}$ toluene (Figure 4.6) under the condition of this low attractant concentration.

\subsection{Implications}

Using the convection-free channel, an accurate methodology was established to evaluate the essential chemotaxis parameters, chemotactic sensitivity coefficient $\left(\chi_{0}\right)$ and chemotactic receptor constant $\left(K_{c}\right)$. Many combinations of chemotactic bacteria and attractants can be tested in the system under different initial attractant concentrations, as long as the attractants do not interact or react with the PDMS material. Combinations of two different attractants/repellents can also be studied by introducing each chemical component into one end of the cross channel. In summary, this methodology can enrich the current database of chemotaxis parameters to a great extent, and is highly recommended to become a common procedure to quantitatively evaluate bacterial chemotaxis. Bacterial random motility and chemical diffusion coefficient can also be measured in this device. The preciseness of the measurement needs to be improved in 
future studies; the enhancement of bacterial fluorescence and the reduction of arc lamp noise are considered as the two main aspects for a more accurate signal detection. 


\section{$5 \mathrm{H}-\mu \mathrm{CHIP}$}

\section{Chapter 5}

\section{H- $\mu \mathrm{CHIP}$}

\subsection{Abstract}

Nonaqueous phase liquid (NAPLs) contaminants are difficult to remove from groundwater due, in part, to the heterogeneous structure in soil. Chemotaxis toward the chemical pollutants, which they degrade, provides a mechanism for bacteria to migrate to locations of high contamination, which may enhance the overall effectiveness of bioremediation. Chemotactic effect on bacterial abundance was studied within the low permeable NAPL-contaminated region in a well-defined porous medium. A heterogeneous microfluidic device was designed to mimic features of the natural contaminated groundwater system. In order to achieve the goal, a new method was developed to trap NAPL residuals (toluene) in low permeable zones, and bacteria were injected into the system with groundwater flow in highly permeable pathways. 
Chemotactic bacteria (P. putida F1) migrated preferentially towards and accumulated in the vicinity of NAPL contaminant sources. The accumulation of chemotactic bacteria was $15 \%$ more by comparison to nonchemotactic controls ( P. putida F1 CheA). Bacteria in the device were also subjected to different flow rates within typical range of groundwater flow rates. Chemotactic bacteria had a generally $5 \%$ more accumulation at $0.5 \mathrm{~m} / \mathrm{d}$ near the intersection between the horizontal channel and the porous network than both the nonchemotactic mutant and bacterial distribution at $5 \mathrm{~m} / \mathrm{d}$. The breakthrough curves observed at the outlet provided indirect evidence that chemotactic bacteria intended to retain in contaminated and low permeable region for a longer time than the nonchemotactic bacteria at $0.25 \mathrm{~m} / \mathrm{d}$. A higher flow rate at $5 \mathrm{~m} / \mathrm{d}$ diminished this retention due to chemotaxis. Mathematical models and related computational fittings yielded outcomes that were consistent with the experimental results, and statistical analysis also supported the experimental comparisons. There is no direct observation on biodegradation, but because toluene is degradable by $P$. putida F1, the experimental observations of biased accumulation of chemotactic bacteria around the NAPL sources within the low permeable region will lead to an increase of contaminant consumption, which can improve the efficiency of bioremediation.

\subsection{Introduction}

The main purpose of this chapter is to present all the results achieved in the heterogeneous porous microfluidic device (H- $\mu$ Chip).

Section 5.3 summarizes all the experimental setup for this study, including the process for trapping toluene droplets in the low permeable region, the device operating procedures and the fitting setup. Section 4.4 discusses all the main results from the H- 
$\mu$ Chip. Toluene ganglia are trapped in designated locations of the porous network as contaminant sources, and $P$. putida's chemotactic response to this chemoattractant is studied under three different circumstances. Firstly, accumulation induced by bacterial chemotaxis is observed at the intersection area between the porous network and the horizontal channel at different flow rates, which indicates a biased movement of chemotactic bacteria toward the contaminant sources. Secondly, bacterial chemotaxis was observed in the vicinity of toluene ganglia under no flow condition, and this result provided direct evidence of chemotaxis to NAPL component in porous structure, and in addition, this experimental setup can be used as an alternative method for NAPL study in agarose plug assay. Thirdly, the breakthrough curves of chemotactic and nonchemotactic bacteria are compared in the $\mathrm{H}-\mu \mathrm{Chip}$ under different flow rates. In section 4.5 the implication of the study on in-situ bioremediation is discussed.

\subsection{Experimental Section}

\subsubsection{Bacteria/Attractant System}

Pseudomonas putida and toluene was the model system for chemotaxis toward a NAPL attractant. As a brief summary from Chapter 3 Materials and Methods, Pseudomonas putida F1 (wild type) was obtained from C. S. Harwood (Parales et al., 2000) and nonchemotactic mutant $P$. putida F1 CheA from R. E. Parales (Liu et al., 2009). An aliquot ( $300 \mu \mathrm{L}$ for $P$. putida) of frozen stock at $-80{ }^{\circ} \mathrm{C}$ was used to inoculate $100 \mathrm{~mL}$ of the modified Hutner's Mineral Base in a sealed $200 \mathrm{~mL}$ glass bottle. $30 \mu \mathrm{L}$ toluene was added as the carbon source for bacteria into the gas phase of the growth media. Oxygen (GT\&S, oxygen compressed USP) was injected at 5 psi for 10 minutes to 
ensure a sufficient supply. When the optical density (measured at $590 \mathrm{~nm}$ in Beckman Coulter, DU 730) reached 0.80 (corresponding to the mid-exponential phase of growth), bacteria were harvested and resuspended in 10\% Random Motility Buffer (RMB) to a density similar to the one when harvested.

P. putida F1 was then dyed with the vital membrane stain FM 4-64 (Invitrogen) at a concentration of $5 \mu \mathrm{g} / \mathrm{mL}$ for 10 minutes and $\mathrm{F} 1 \mathrm{CheA}$ was dyed with $100 \mathrm{mg} / \mathrm{L}$ DAPI (Invitrogen) for 30 minutes, and both bacterial suspensions were washed twice and then mixed for later experimental use. The bacteria suspension was also mixed with $1.2 \mathrm{mM}$ sodium nitrate (Sigma-Aldrich, ACS reagent) as electron acceptor to maintain bacterial motility (Bauer et al., 2008). $7 \mathrm{~mL}$ pure toluene (Sigma-Aldrich, HPLC grade) was dyed with $1 \mathrm{mg}$ Oil Red O (FisherBiotech, electrophoresis grade) to show observable red color.

\subsubsection{Toluene Trapping}

$70 \%$ ethanol (LabChem Inc., 70\% v/v reagent) was first injected into the microfluidic device from both the bacterial and NAPL inlets, in order to saturate the system. Because air is soluble in ethanol, the air bubbles in the system were eliminated quickly by continuous high-pressure ethanol flow. DI water was then injected into the microfluidic device to displace the ethanol, and the microfluidic system was kept saturated during the whole experiment. Red dyed toluene was injected from the NAPL inlet at the bottom of Figure 5.1 to replace the water in the fine network, and then DI water was injected again to wash out the toluene in the fine network, while toluene droplets remained in all the ganglia, as illustrated in Figure 5.1. 


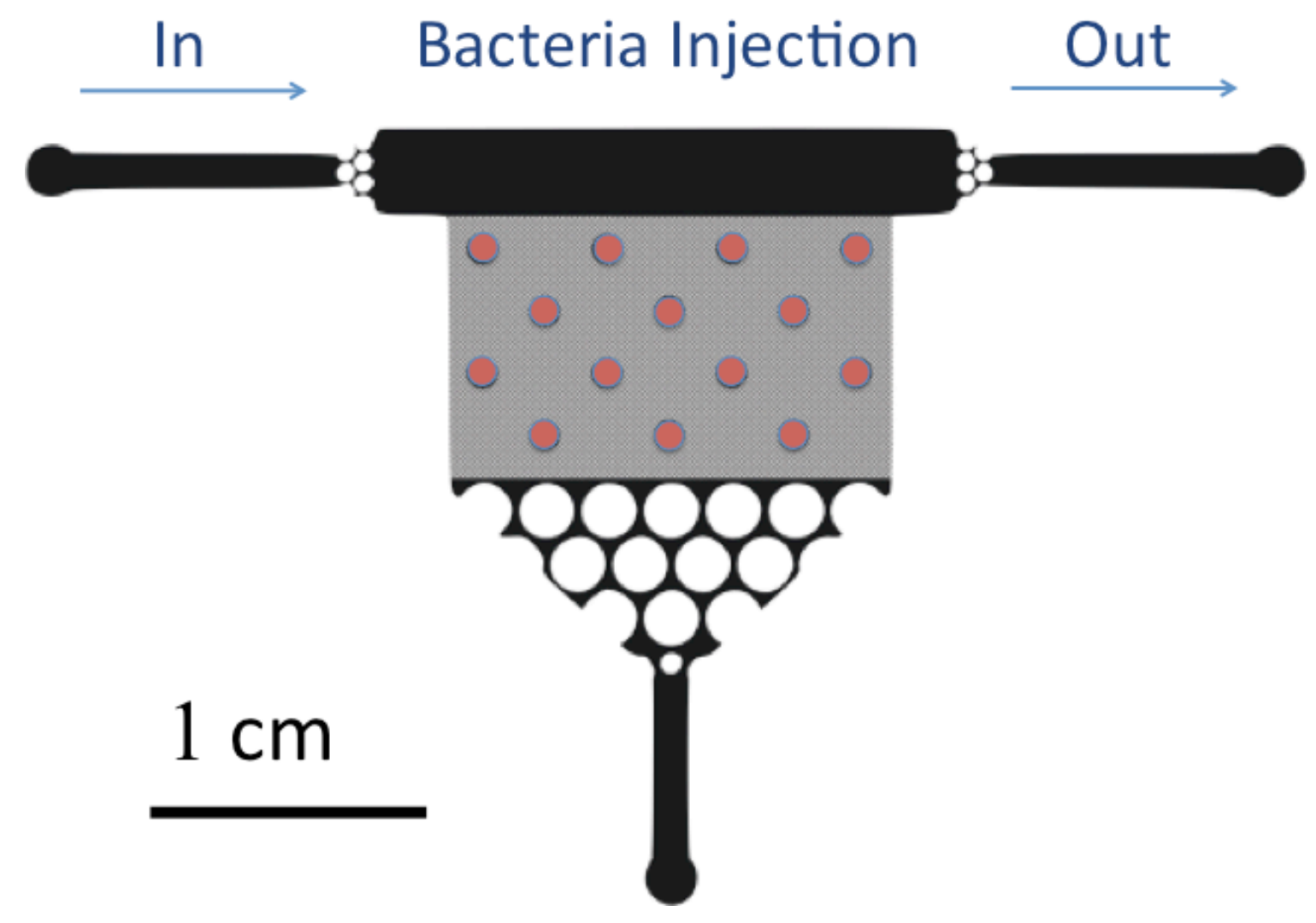

Figure 5.1 Schematic illustration of the bacterial injection in $\mathrm{H}-\mu \mathrm{Chip}$. The red color represents the toluene ganglia, and the arrows indicate the inlet, the outlet and the flow direction for bacteria.

\subsubsection{Continuous Injection}

The bacterial suspension was injected into the main channel by $100 \mu \mathrm{L}$ point-style syringes (Spectrum Laboratory Products Inc.), and then the syringes were fixed onto a syringe pump (PHD 2000 Infusion, Harvard Apparatus) and pushed at different speeds, which were comparable to 0.5 and $5 \mathrm{~m} / \mathrm{d}$ in the main channel.

This study was conducted to examine the bacterial distribution at the interface between the horizontal channel and the porous network at the different flow rates, 0.5 and $5 \mathrm{~m} / \mathrm{d}$. Quantitative analyses were made for comparisons between different flow rates, 
and between chemotactic and nonchemotactic bacteria. These two different values of flow velocity were chosen because they are within the typical range of groundwater flow rate from 0.1 to $10 \mathrm{~m} / \mathrm{d}$, and each of the values can be representative of the slow and fast flows, respectively. Bacterial suspension was continuously injected at certain flow rate. Preliminary simulation showed that the system reached the steady state after two hours of injection at $0.5 \mathrm{~m} / \mathrm{d}$, and after half an hour at $5 \mathrm{~m} / \mathrm{d}$ based on Brinkman equation and advection-dispersion equations (Eqn 3.2-3.4). Images on bacterial fluorescence were also taken in all the channel spaces at the time points as mentioned above, and results showed that bacteria occupied all spaces after two hours at $0.5 \mathrm{~m} / \mathrm{d}$ and half an hour at $5 \mathrm{~m} / \mathrm{d}$, and the densities in the horizontal wide channel were the same as those in the thin channels deep in the porous network. This observation was done with unstained bacteria, and the unstained bacterial density was recorded as the intensity of scattered light from bacteria in bright field; this method was described in details and validated by Wang et al. (2012). This study was done in the original H- $\mu$ Chip, which was shown in Figure 2.6. Because the device had eight opening junctions at the interface between the porous network and the horizontal channel, so bacterial density at each of the junctions was recorded in terms of light intensity. The predicted distribution of attractant concentration in the horizontal channel of the original $\mathrm{H}-\mu \mathrm{Chip}$ was compared with that in the new $\mathrm{H}-\mu \mathrm{Chip}$ (Figure 5.1), and the attractant concentrations were compared along the centerline of the horizontal channel and three evenly distributed transverse lines, as indicated by the red lines in Figure 5.2. The positions of the transverse lines corresponded to three openings between the horizontal channels and the porous matrix in the original design. 


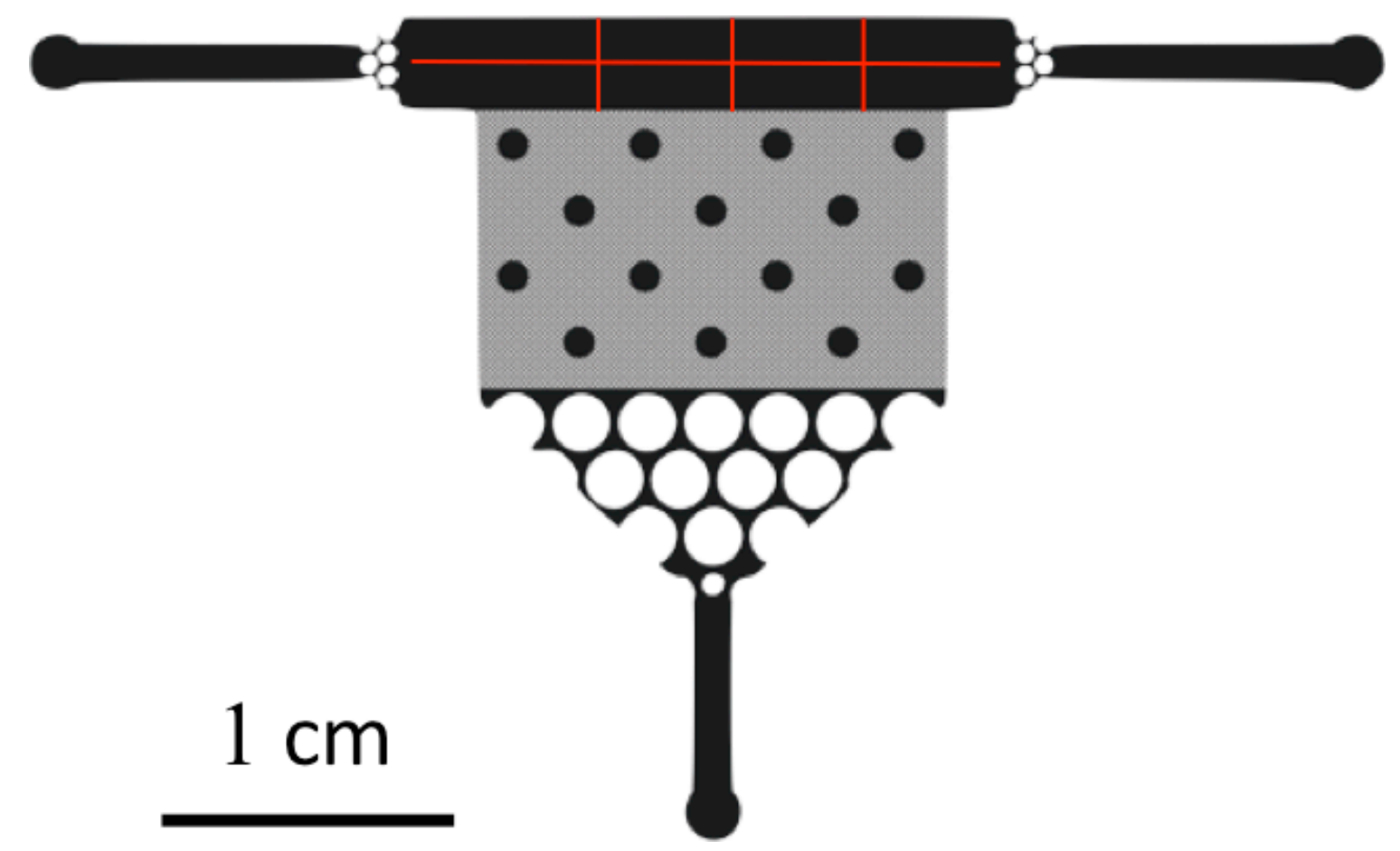

Figure 5.2 The predicted attractant concentrations for both the original and the new H- $\mu$ Chip in the horizontal channel were compared along the red lines in the image.

\subsubsection{No Flow}

The flow stopped when bacterial density in porous network equaled to that in main channel by visual observation, and after the whole system became stationary for 10 minutes, the bacterial distribution in vicinity of the toluene ganglia was recorded under the microscope. This step mimicked the protocol to observe $P$. putida distribution in plug assay (Toepfer et al., 2012). 30 images were taken in different channels of the porous network near the edge of ganglia, and then were averaged for image analysis.

\subsubsection{Breakthrough Trends}

The other study was to wash out the bacteria mixture at the different flow rates, 0.25 and $5 \mathrm{~m} / \mathrm{d}$. These two different values of flow velocity were chosen because they are 
within the typical range of groundwater flow rate from 0.1 to $10 \mathrm{~m} / \mathrm{d}$, and each of the values can be representative of the slow and fast flows, respectively. Then the injectate fluid was changed from bacteria to $10 \%$ random motility buffer, and the speed was reduced to 0.25 or $5 \mathrm{~m} /$ day to wash the bacteria out of the microfluidic device for 18 continuous hours. Images of the fluorescent bacteria at the outlet were taken and collected during this process. Bacterial density was then recorded in term of fluorescent intensity at different time points, and was normalized with initial intensity in the main channel at certain speed. Observation was done at the outlet of the main channel. The image analysis for both studies followed the same protocol in Wang et al. 2012.

\subsubsection{Fitting Setup}

The two-dimensional profiles of flow and attractant/bacterial distribution were solved using Equation $3.2-3.6$. The equations and boundary conditions might subject to slight changes for different experimental requirements.

\subsection{Results and Discussion}

\subsubsection{Toluene Trapping}

Red dyed toluene was injected from the NAPL inlet to replace water within the fine network and ganglia, and then DI water was injected again to wash out toluene in the fine network, while toluene remained within the ganglia, as shown in Figure 5.3. 


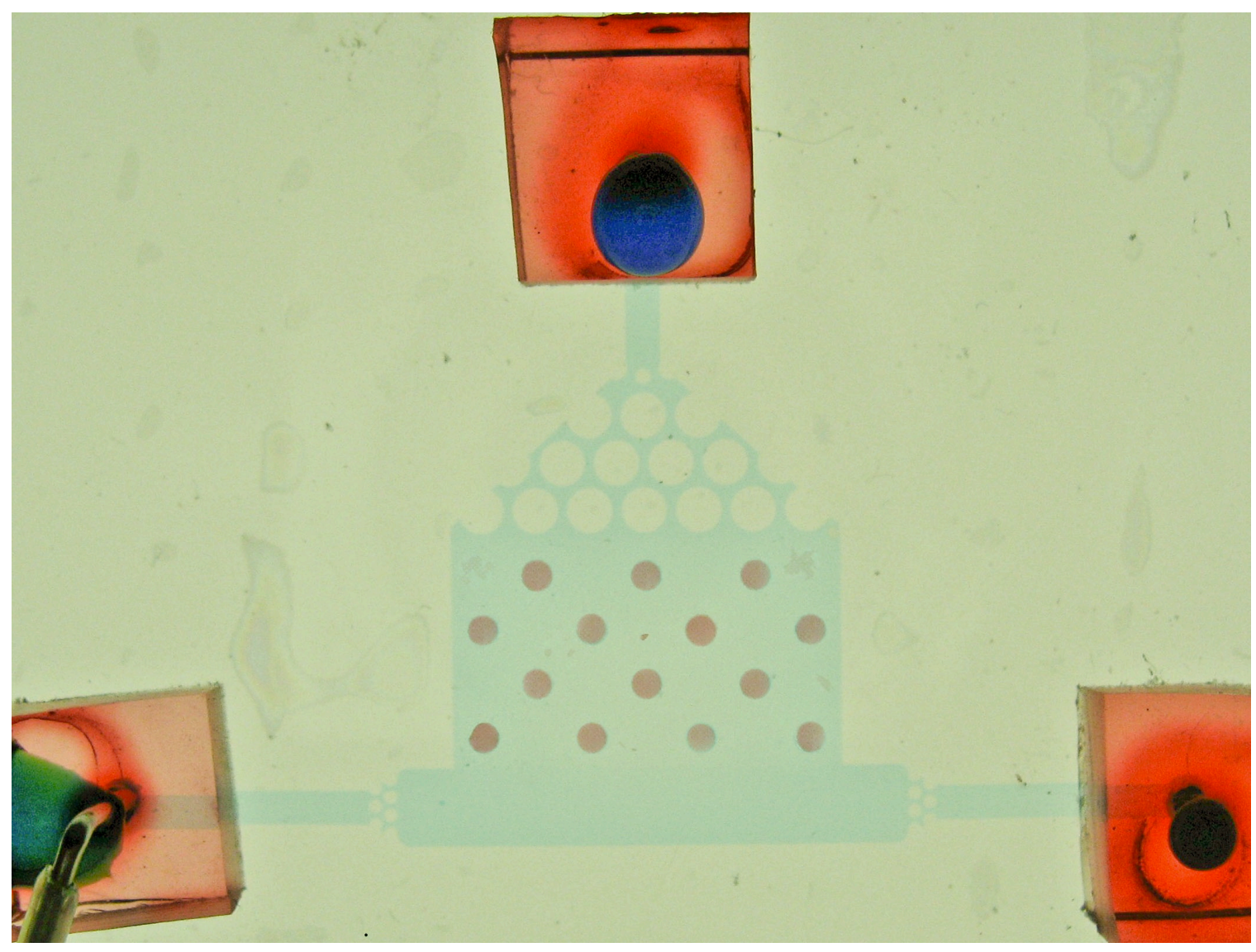

Figure 5.3 Toluene dyed with Oil Red $\mathrm{O}$ was retained as ganglia in the microfluidic device. A light blue colored aqueous solution was also injected to illustrate the channel patterns.

The entrapment of toluene ganglia resulted mainly from the different physical properties between the material and the NAPL chemical: glass (the material of the device) is hydrophilic and toluene is hydrophobic. Therefore, toluene exhibits a high contact angle in the water saturated glass-based spaces and keeps a round intact shape. When water washes toluene out of the porous structure, it flows along the glass surface because both water and glass are hydrophilic, so the toluene residuals in the channels can be carried away by water flow easily due to the their poor attachment to the glass surface; however, the toluene ganglia are difficult to wash out As shown in Figure 5.4 as a 
horizontal view of Figure 3.1c, a thin layer of water surrounds the toluene ganglion, and the toluene ganglion exhibits a high surface tension at the top openings, which generates an overall downward force $\mathrm{F}_{\mathrm{s}}$ on the ganglion. Moreover, the glass wall on top also provides a downward resistance force $\mathrm{F}_{\mathrm{w}}$ on the toluene ganglion, and both forces prevent the organic compound from being washed out. Thus, even though toluene has a lower density than water, toluene remains as ganglia in the bottom layer of the device. This method of trapping NAPL based on surface tension and device's hydrophilicity is new achievement. Wan and Wilson (1994a) successfully retained NAPL in a porous medium, but it was achieved in an unsaturated system and the entrapments were randomly distributed, so the bacterial distribution could be highly affected by adjacent NAPL sources and air bubbles. The method introduced by this study could retain NAPL ganglia in the designated spaces of the saturated porous network, which helped quantify chemotaxis effect toward single NAPL attractant source, and can be widely used in the trapping of nonaqueous phase liquid chemicals in future research.

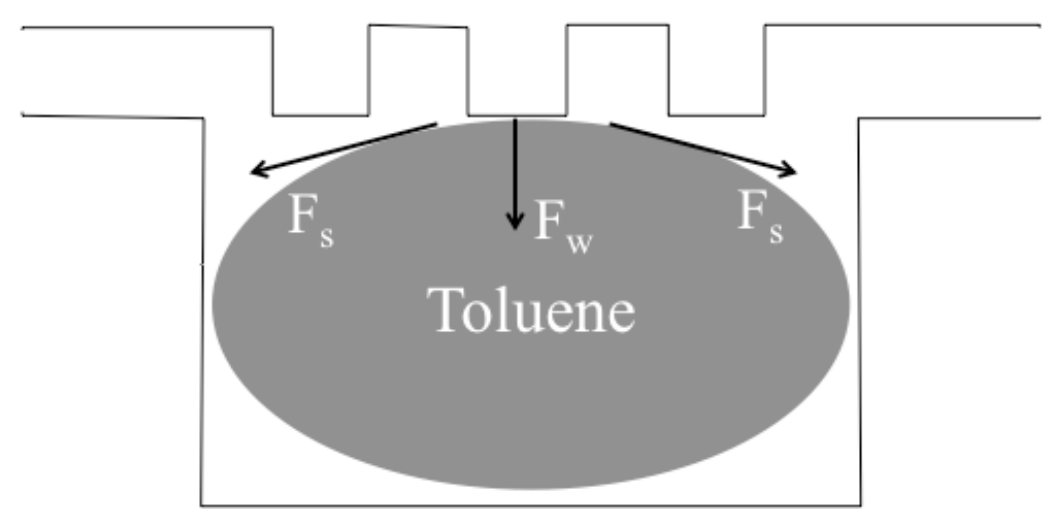

Figure 5.4 Cartoon illustration of the toluene entrapment in $\mathrm{H}-\mu \mathrm{Chip} . \mathrm{F}_{\mathrm{s}}$ stands for the force vector induced from surface tension on a toluene ganglion, and $F_{w}$ represents the resistance force from the glass wall. 
After toluene ganglia were trapped within the circular spaces of the device, a high flow rate was used to test their stability. The toluene ganglia remained stable under injection rates comparable to $100 \mathrm{~m} / \mathrm{d}$ in the main channel and after 24 hours of continuous flow at $5 \mathrm{~m} / \mathrm{d}$. This high level of stability of the toluene ganglia is attributed to the low solubility ( $5.6 \mathrm{mmol} / \mathrm{L})$ and high surface tension between toluene and water. By way of extension to natural systems, these two properties also impact NAPL retention as a pure phase and its distribution within the interstices of the soil matrix. Moreover, as the flow rate was further reduced within the narrow pore spaces, it was more difficult to wash out the residual NAPL. These are contributing factors to why NAPL contaminants remain in the groundwater system for decades.

\subsubsection{Bacterial Accumulation under Continuous Injection}

The continuous injection method (Figure 5.1) simulated the actual bioremediation injection, which mainly happened at the high conductive region instead of low permeable layer in order to avoid clogging and built-up pressure (Gierczak et al., 2006; Lee et al., 2000). Because this experiment was carried out in the original $\mathrm{H}-\mu \mathrm{Chip}$, in which the porous structure was coarser and the pores had a larger size (Figure 2.6), several preliminary simulation were conducted to test whether the results from the original $\mathrm{H}$ $\mu$ Chip could be considered good indicators of expected behavior in the new device. The observation of bacterial accumulation with the original H- $\mu$ Chip occurred within the horizontal channel, so we compared the velocity field and attractant distribution within the horizontal channels of the two microfluidic devices. We reasoned if they were 
comparable, then the results from the original $\mathrm{H}-\mu \mathrm{Chip}$ could be used to indicate what would also happen in the new H- $\mu$ Chip. As discussed in Appendix E, the velocity fields in the horizontal channel were almost exactly the same for both designs. A comparison of the attractant distributions is presented in Figure 5.5 and 6.6. Both attractant distributions followed a similar increase trend along the centerline of the horizontal channel. The increase was also nearly linear, and the attractant concentration for the new H- $\mu$ Chip was slightly lower than that for the original design at each location. This is because the finer porous structure helped to retain NAPL concentration, so that less of this chemical could be brought into the wide horizontal channel; this is also close to the real NAPL contamination problem, where NAPL residuals could remain in the fine porous media for decades. Although there was a slight difference in the attractant distribution, the ratio of the biggest difference value to the local concentration in the new H- $\mu$ Chip was only $4.3 \%$, so the difference was fairly small on the horizontal direction. 


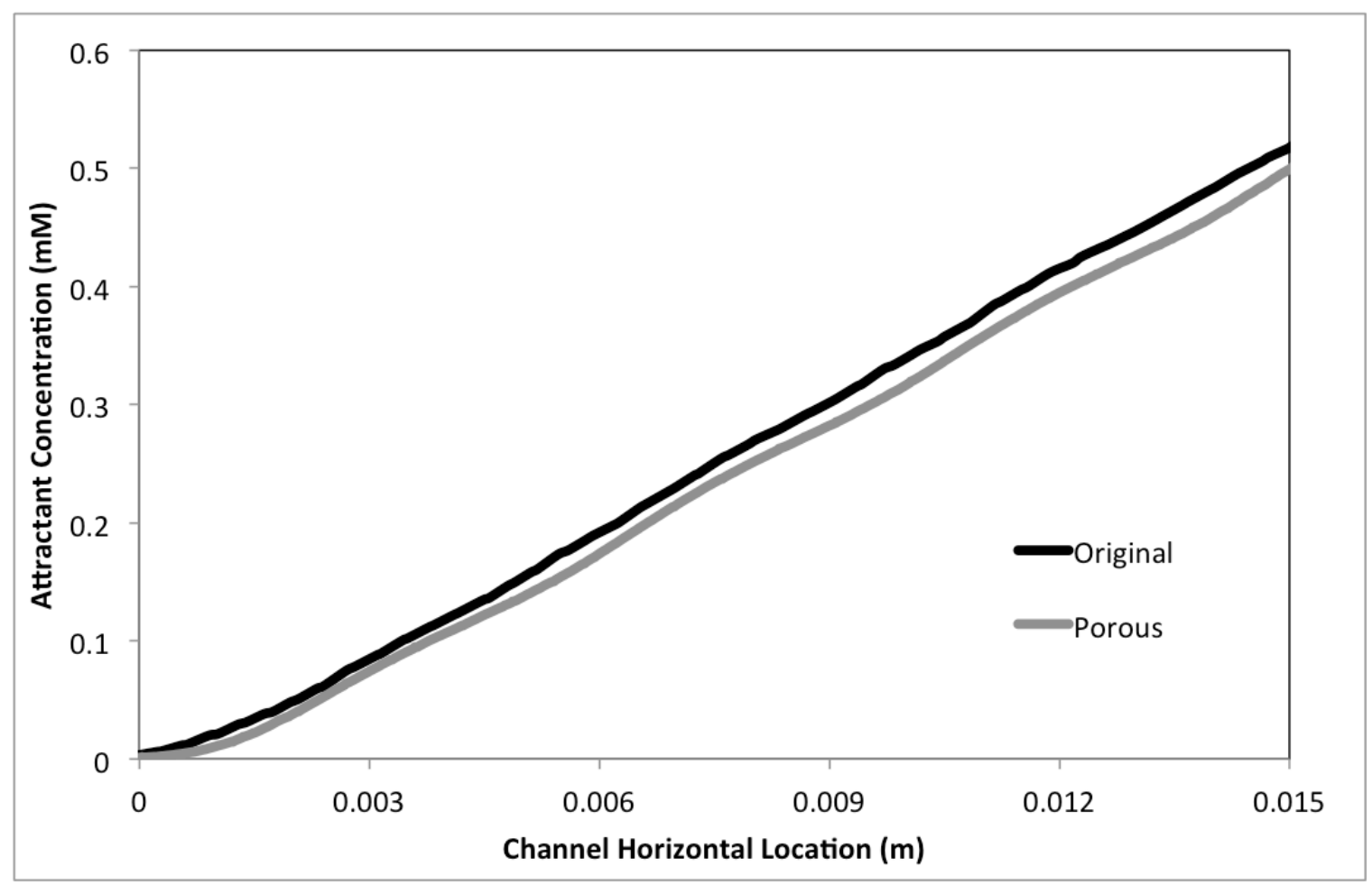

Figure 5.5 The simulated attractant distribution along the centerline in the horizontal channel for both the original (black) and the new (gray) $\mathrm{H}-\mu$ Chip at $0.5 \mathrm{~m} / \mathrm{d}$.

Attractant distributions in the direction transverse to flow are compared in Figure 5.6. The distributions in different microfluidic devices at each position overlap each other. The location at $0 \mathrm{~m}$ corresponds to the intersection between the horizontal channel and the porous structure, and the bacterial distribution at each opening was observed in a $500 \mu \mathrm{m}$ by $500 \mu \mathrm{m}$ region of interest next to the intersection in the horizontal channel.

Figure 5.6 shows that the trends follows linear distributions in the range of 0 to $500 \mu \mathrm{m}$, and the slopes are also similar to each other. In fact, the averaged concentration at the openings in $\mathrm{H}-\mu \mathrm{Chip}$ is $4.6 \pm 0.4 \%$ higher than that in the original design, which is also the largest difference at all locations, and the slopes from 0 to $500 \mu \mathrm{m}$ in $\mathrm{H}-\mu \mathrm{Chip}$ are $2.3 \pm 0.2 \%$ higher than that in the original design. The chemotactic velocity is related to 
this values, as describes in Eqn 3.6. If $K_{c}$ is set as $1 \mathrm{mM}$ for $P$. putida $\mathrm{F} 1$ and toluene, the calculated chemotactic velocity in $\mathrm{H}-\mu$ Chip is approximately $2.1 \%$ lower than that in the original design; the difference is relatively small and can be neglected. Overall, the attractant distributions had many similarities in the horizontal channel for both microfluidic devices, so a bacterial accumulation near the intersection in the original design could also indicate a similar accumulation in the new $\mathrm{H}-\mu \mathrm{Chip}$, which might be a bit larger than the former because of the plateau problem in the former device.

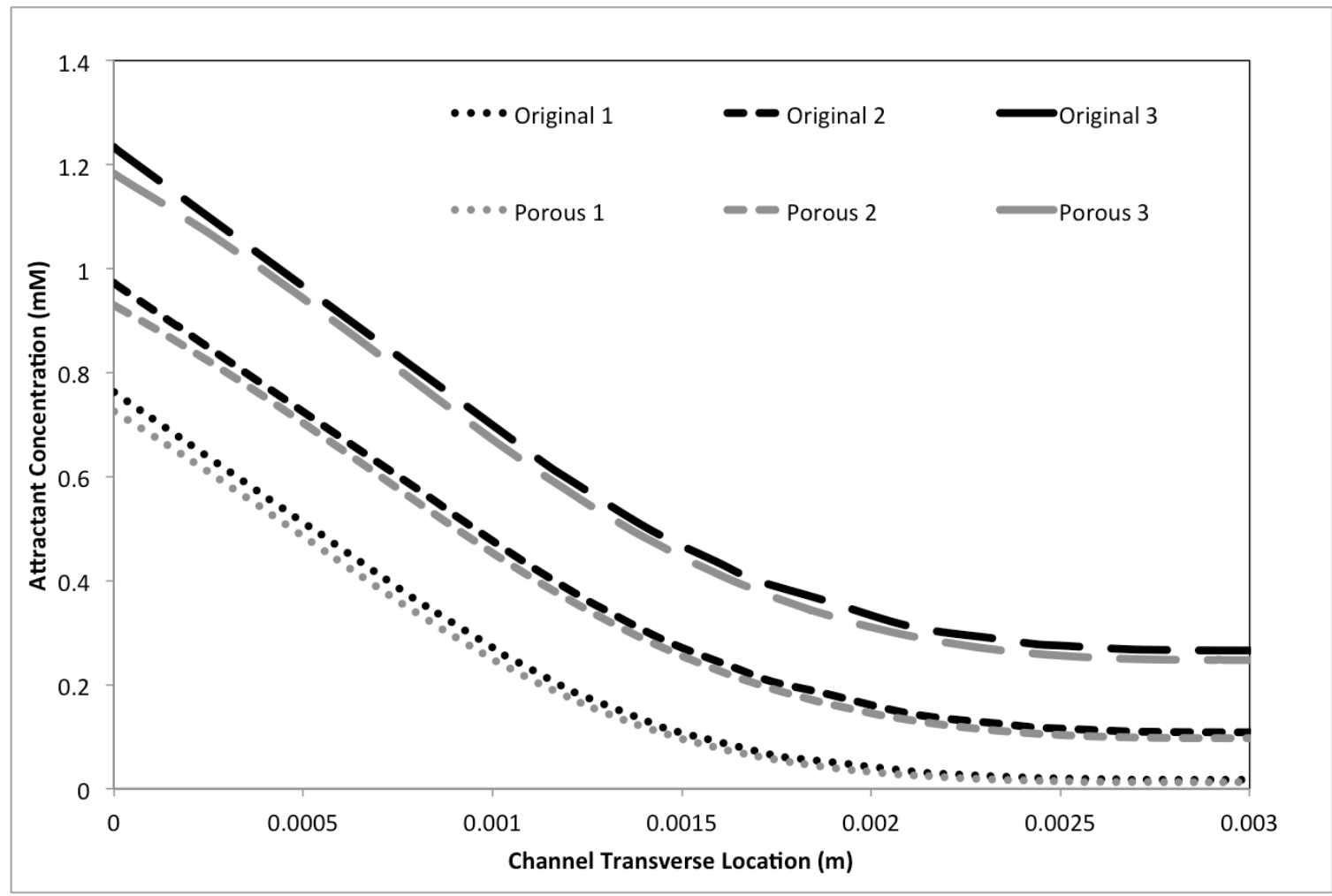

Figure 5.6 The simulated attractant distribution along the transverse direction in the horizontal channel for both the original (black curves) and the new (gray curves) H- $\mu$ Chip at $0.5 \mathrm{~m} / \mathrm{d}$. Number 1 to 3 increases from left to right in Figure 5.2, as the flow is injected from the inlet on left. 
The results about bacterial accumulation under continuous injection in the original H- $\mu$ Chip are shown in Figure 5.7 and 6.8. Chemotactic and nonchemotactic bacteria are compared in Figure 5.7, and chemotactic bacteria are compared at two different fluid velocities in Figure 5.8.

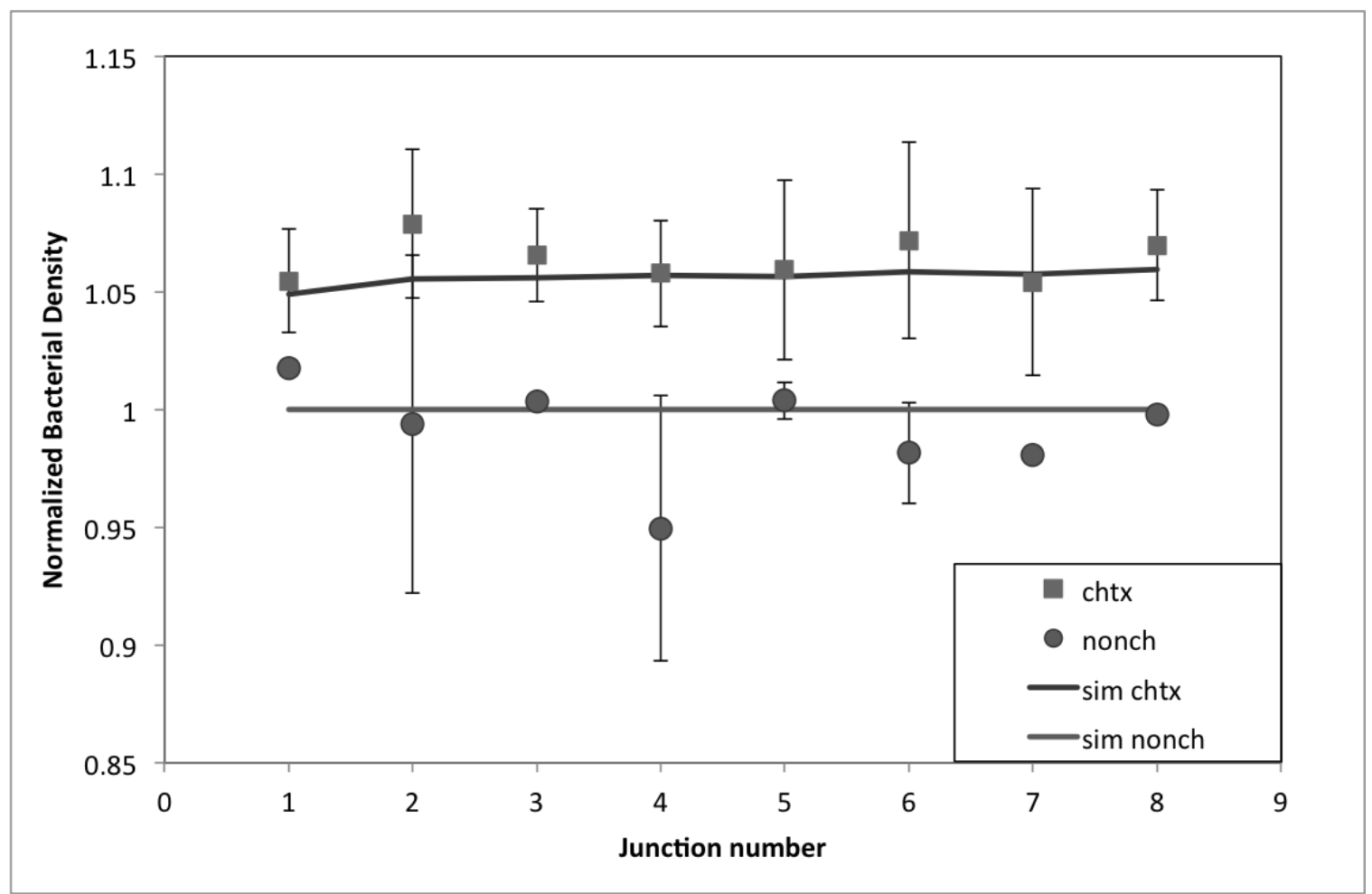

Figure 5.7 Distribution of chemotactic (light gray square) and nonchemotactic (dark gray circle) bacteria at different junctions with a fluid velocity of $0.5 \mathrm{~m} / \mathrm{d}$. The connecting lines indicate the fitted data for accumulation of chemotactic bacteria at the junctions, and the fitted results for nonchemotactic bacteria respectively. 


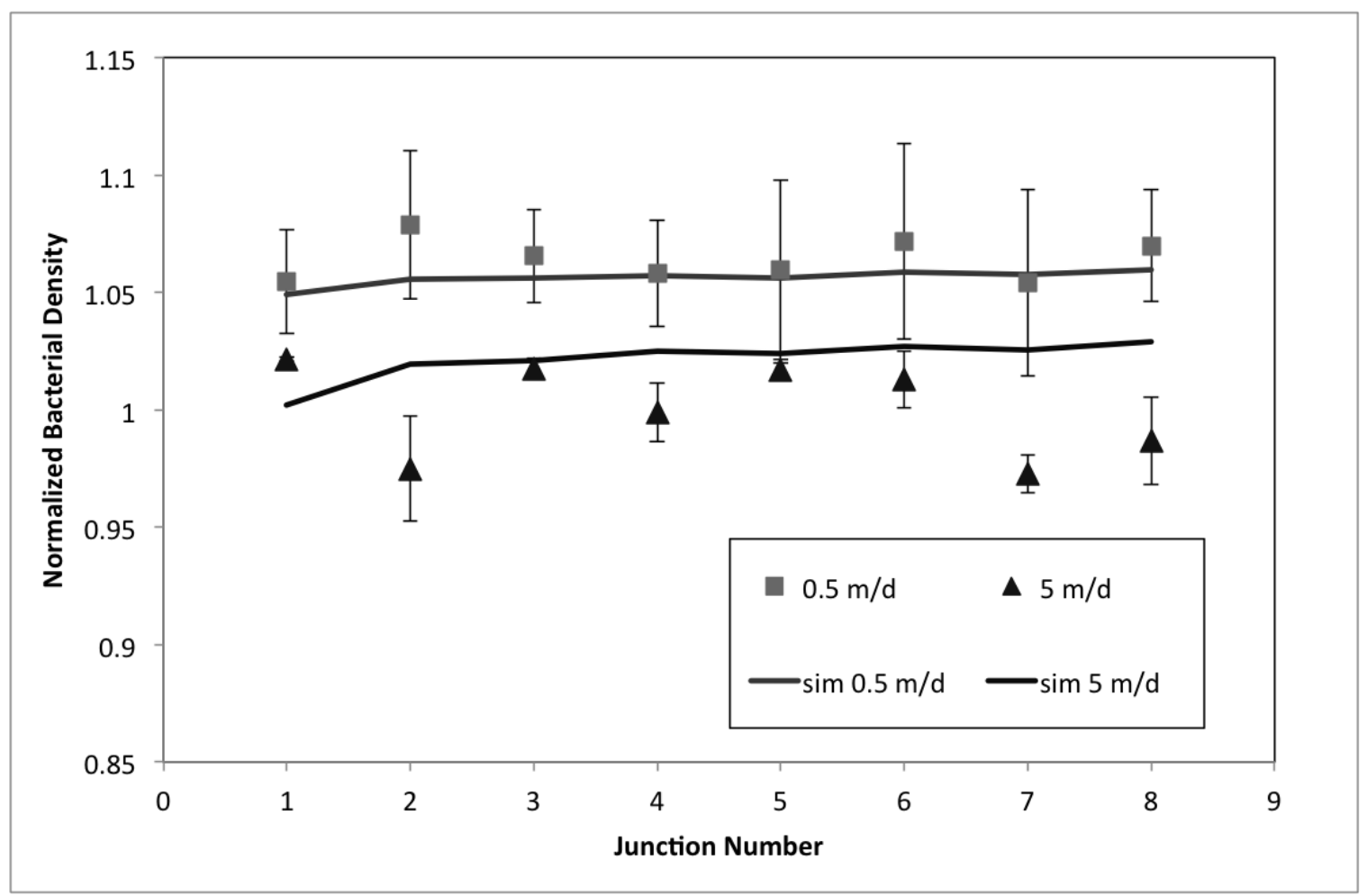

Figure 5.8 Distribution of chemotactic bacteria at different junctions under different convective flow rates, which are $0.5 \mathrm{~m} / \mathrm{d}$ (gray square) and $5 \mathrm{~m} / \mathrm{d}$ (black triangle) respectively. The connecting lines indicate the fitted data for bacterial accumulation at the junctions with flow rate of $0.5 \mathrm{~m} / \mathrm{d}$, and the fitted results for that at $5 \mathrm{~m} / \mathrm{d}$ respectively.

Greater accumulation of chemotactic bacteria was observed at all the junctions, when compared with nonchemotactic bacteria, as indicated in Figure 5.7. This figure also shows that the distribution of both chemotactic and nonchemotactic bacteria followed the trend predicted by the mathematical models with the parameters fitted from the previous chapter, which are listed in Table 6.1. The accumulation of chemotactic bacteria near the junctions decreased at a higher convective flow rate of $5 \mathrm{~m} / \mathrm{d}$ (Figure 5.8), which is supported by the previous experimental results (Wang et al., 2012); Wang and coworkers reported that a transverse bacterial accumulation was observed in the vicinity of a NAPL 
interface at the slow flow rate of $0.5 \mathrm{~m} / \mathrm{d}(5.8 \mu \mathrm{m} / \mathrm{s})$, which is smaller than the bacterial swimming speed of $44 \mu \mathrm{m} / \mathrm{s}$, but the accumulation decreased dramatically when the flow rate increased to $5 \mathrm{~m} / \mathrm{d}$, which is higher than the bacterial swimming speed. A smaller convective flow left more retention time for the motile bacteria to react to the attractant source on the transverse direction. All experimental data were compared statistically with two-way ANOVA test, and the test showed that the chemotaxis group at $0.5 \mathrm{~m} / \mathrm{d}$ is significantly different from either the nonchemotaxis control or the chemotaxis group at 5 $\mathrm{m} / \mathrm{d}$, with a $\mathrm{p}$ value smaller than 0.0001 in both cases. Such difference was not found when the nonchemotaxis group was compared with the $5 \mathrm{~m} / \mathrm{d}$ group with $\mathrm{p}=0.1492$, which implies that transport by convection dominated microbial motility and chemotaxis.

These results suggested that the chemotactic bacteria carried along flow in the macropore (horizontal channel) sensed the concentration gradient from the NAPL attractant trapped in the low permeable region (porous network), and accumulated in the vicinity of the junctions between these two regions, which led to a build-up in bacterial population near the contaminant sources. Because the rate of biodegradation is proportional to the bacterial population density, we expect this accumulation of bacteria will enhance the biodegradation rate. However, as the groundwater velocities were increased from 0.5 to $5 \mathrm{~m} / \mathrm{d}$, the accumulation of chemotactic bacteria diminished from the NAPL sources. Model equations solved using COMSOL also supported the experimental findings about the microbial behaviors of $P$. putida strains in presence of toluene as chemoattractant. 


\subsubsection{No Flow in the H- $\mu$ Chip}

Bacteria were transported into the fine porous network under high convective velocities in the main channel, and the convective flow from the high permeable region adjacent to the porous structure carried bacteria into the narrow pore spaces in the low permeable region. This observation was also supported by the prediction from model equations within the COMSOL software routines, where the essential parameter for the porous structure permeability $\kappa_{b r}\left(1.0 \times 10^{-9} \mathrm{~m}^{2}\right)$ was in the typical range of $10^{-8}$ to $10^{-11} \mathrm{~m}^{2}$ for sand and gravel (Bear, 1988). Once bacteria occupied the entire porous network, the flow was stopped. Ten minutes after the flow was stopped, images of the bacterial distribution near toluene ganglia were recorded. Figures 5.9 and 5.10 show the bacterial distribution within pores at the edge of toluene ganglia under this condition. The cartoon in Figure 5.9a illustrates the expected bacterial distribution near one ganglion. Figure 5.9b shows a single image of the actual bacterial distribution and Figure 5.9c represents a composite of 30 images taken at different locations around the ganglion. The values of bacterial density in Figure 5.10 were normalized by the initial value in the main channel at injection. Accumulation of P. putida F1 increased as the distance from the toluene source decreased, while nonchemotactic bacteria were distributed evenly over the same distances and no accumulation was evident. This indicated that chemotactic bacteria responded to the concentration gradient of the attractant and migrated toward the regions where the toluene concentration was higher, and conversely, the nonchemotactic mutant did not present any observable response to the gradient profile. The bacterial density of chemotactic bacteria was approximately $15 \%$ greater at the visual edge of the toluene sources than at a distance of $500 \mu \mathrm{m}$ away from the sources. 


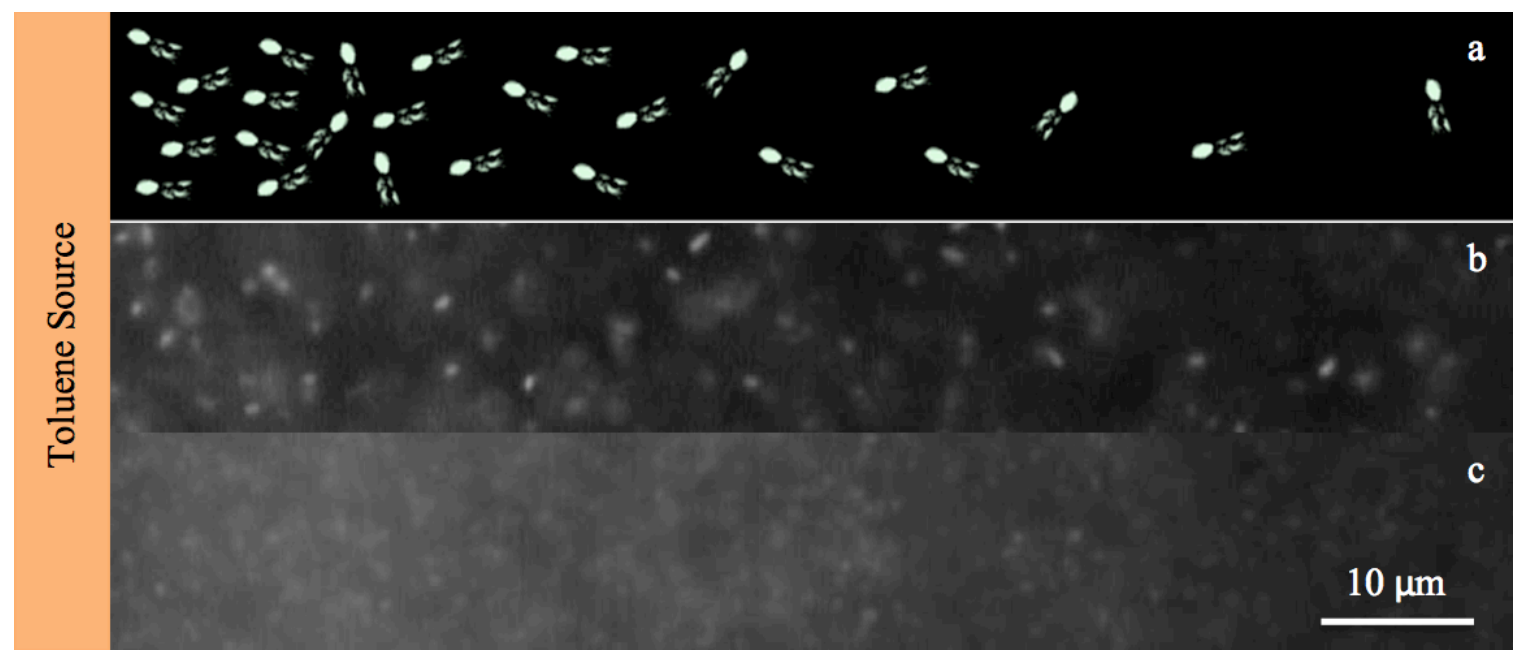

Figure 5.9 (a) Cartoon illustration and (b) image of chemotactic bacteria near a NAPL source. Each individual bright spot in (b) is a fluorescent bacterium dyed by FM 4-64, and (c) is a composite average from over 30 images of (b) at different locations around the toluene-water interface of a single ganglion. 


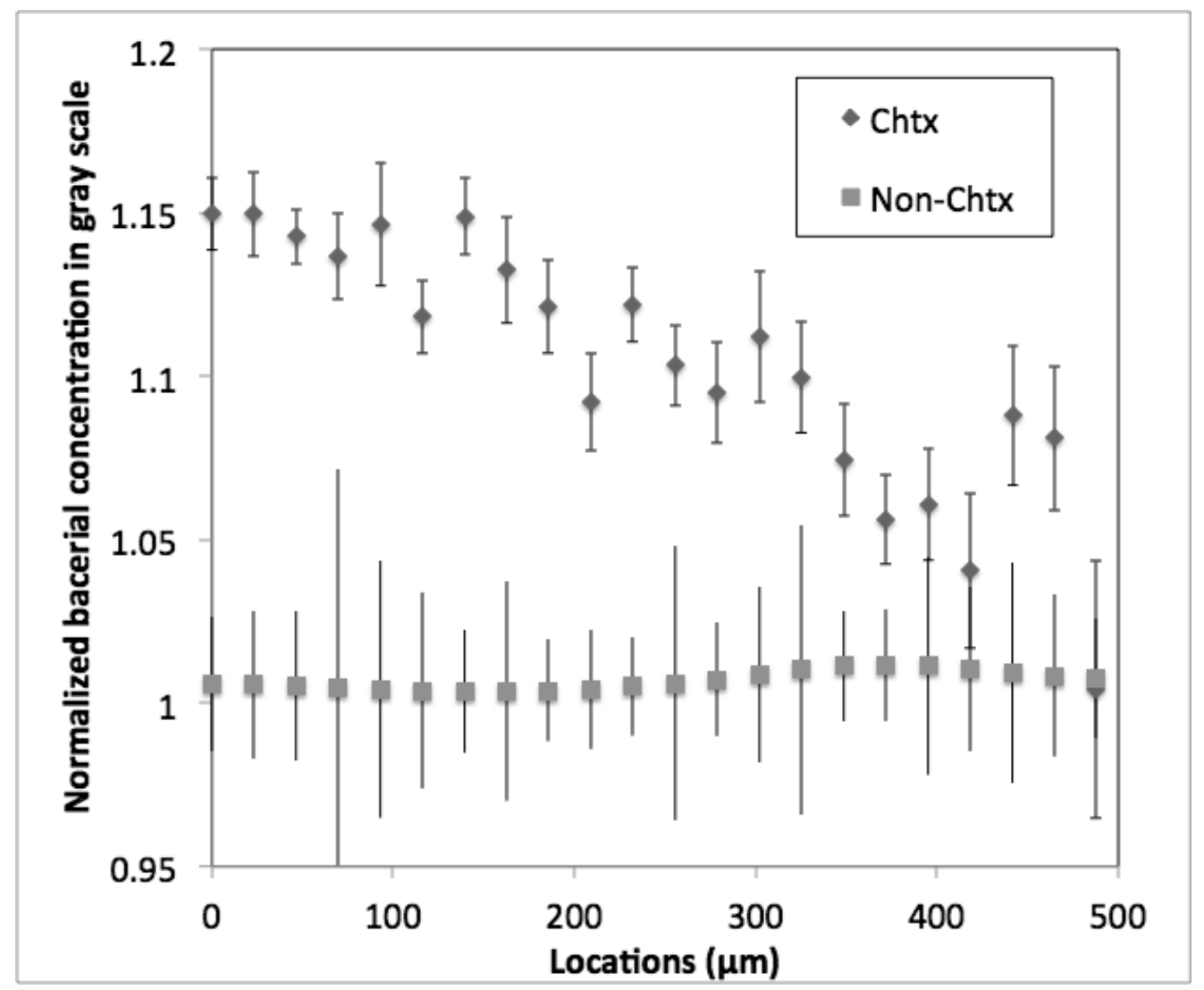

Figure 5.10 The distribution of chemotactic and nonchemotactic bacteria near NAPL sources.

The differences in the distribution between the wild type and the mutant are statistically significant in the range of $0-300 \mu \mathrm{m}$, with $\mathrm{p}<0.0001$ by the two-way ANOVA. The results of both chemotactic accumulation and statistical analysis are consistent with what were reported from previous studies in the single-pore device (Wang et al., 2012). However, the previous study only measured chemotaxis near one single pore slit; this study successfully captured similar results in a porous structure, designed to mimic a more complex groundwater system with contaminant sources.

The reason why the nonchemotactic bacteria results have bigger error bars than those of chemotactic ones is due to the difference between fluorescence intensities. DAPI was used to stain nonchemotactic bacteria, and its fluorescence intensity was lower than 
FM 4-64 and GFP, so its signal-to-noise ratio was also lower compared to the other fluorescence label, and that is why the error bars of nonchemotactic bacteria are bigger than the chemotactic ones.

Features of this microfluidic device resemble aspects of the agarose plug assay of Yu and Alam (1997). The plug assay has been widely used as a screening tool for bacterial chemotaxis (Parales et al., 2000; Parales and Harwood, 2002; Grimm and Harwood, 1999; Toepfer et al., 2012). One disadvantage of the assay is that properties of the agarose plug, which serves as the source of chemoattractant, limits the choices of chemicals that can be tested. NAPL chemicals are not very soluble in the aqueous agar solution. The reason why toluene is not recommended in plug assay is not only because of its low solubility in the agarose gel, but also because this organic chemical is highly volatile. When toluene is added to the hot melted agar solution during the process of making the central plug, an uncertain amount of toluene can evaporate quickly; thus, the toluene concentration in the agar is difficult to control. Furthermore, motile bacteria interact with and accumulate near the agar surface in a plug assay (Liu and Ford, 2009; Kusy and Ford, 2009), which may complicate analysis of the bacterial distribution from a mathematical model. The NAPL ganglia within the microfluidic system presented in this study provide an alternative approach, which addresses these issues.

\subsubsection{Bacterial Breakthrough Curves in the H- $\mu$ Chip}

Figure 5.11 shows that a lower density of chemotactic bacteria was detected at the outlet at $0.25 \mathrm{~m} / \mathrm{d}$ than the nonchemotactic mutant in $\mathrm{H}-\mu \mathrm{Chip}$, as the normalized density of chemotactic bacteria dropped to about zero after 18 hours washout, and that of 
nonchemotactic mutant still remained at a value close to 0.1 . This result indicated that more chemotactic bacteria remained in the microfluidic device than nonchemotactic bacteria at the slow flow rate. Because chemotactic $P$. putida F1 was stained with membrane dye FM 4-64, and the bacteria could immediately lose their motility once FM 4-64 was excited to show fluorescence (Appendix E), so direct observation on bacterial distribution was not allowed inside the porous network. Therefore, mathematical models were solved to support the statement above by determining the change in bacterial density remaining in the device on as a function of time. Numerical solution showed that the bacterial suspension in the horizontal channel was washed out quickly after one hour of buffer injection at $0.25 \mathrm{~m} / \mathrm{d}$, which could also be indicated by the plateau concentration of attractant at unit 1 for the first hour of injection, and the remaining bacterial population was within the porous network. Moreover, the average bacterial density in the porous network (not shown in the figure) was $60.7 \%$ of the inlet density for chemotactic bacteria after three hours of buffer wash at $0.25 \mathrm{~m} / \mathrm{d}$, and $53.6 \%$ for nonchemotactic bacteria under the same conditions. After 18 hours of wash out, the percentage of the remaining chemotactic bacteria dropped to $21.0 \%$, and the value for nonchemotactic bacteria decreased to $15.4 \%$. According to the model equations, chemotactic bacteria responded to the transverse gradient of attractant within the porous structure, and the induced chemotaxis effect redirected the bacterial population to migrate away from the macrochannel, thus, the bacteria remained in the "contaminant layer" for a longer time. All experimental data were also compared by two-way ANOVA tests, which show that there exists a significant difference between chemotactic and nonchemotactic groups at $0.25 \mathrm{~m} / \mathrm{d}$ with $\mathrm{p}<0.0001$. The difference was not only shown by the experimental data, 
but also predicted by the mathematical models solved using a finite element algorithm in COMSOL (Figure 5.11).

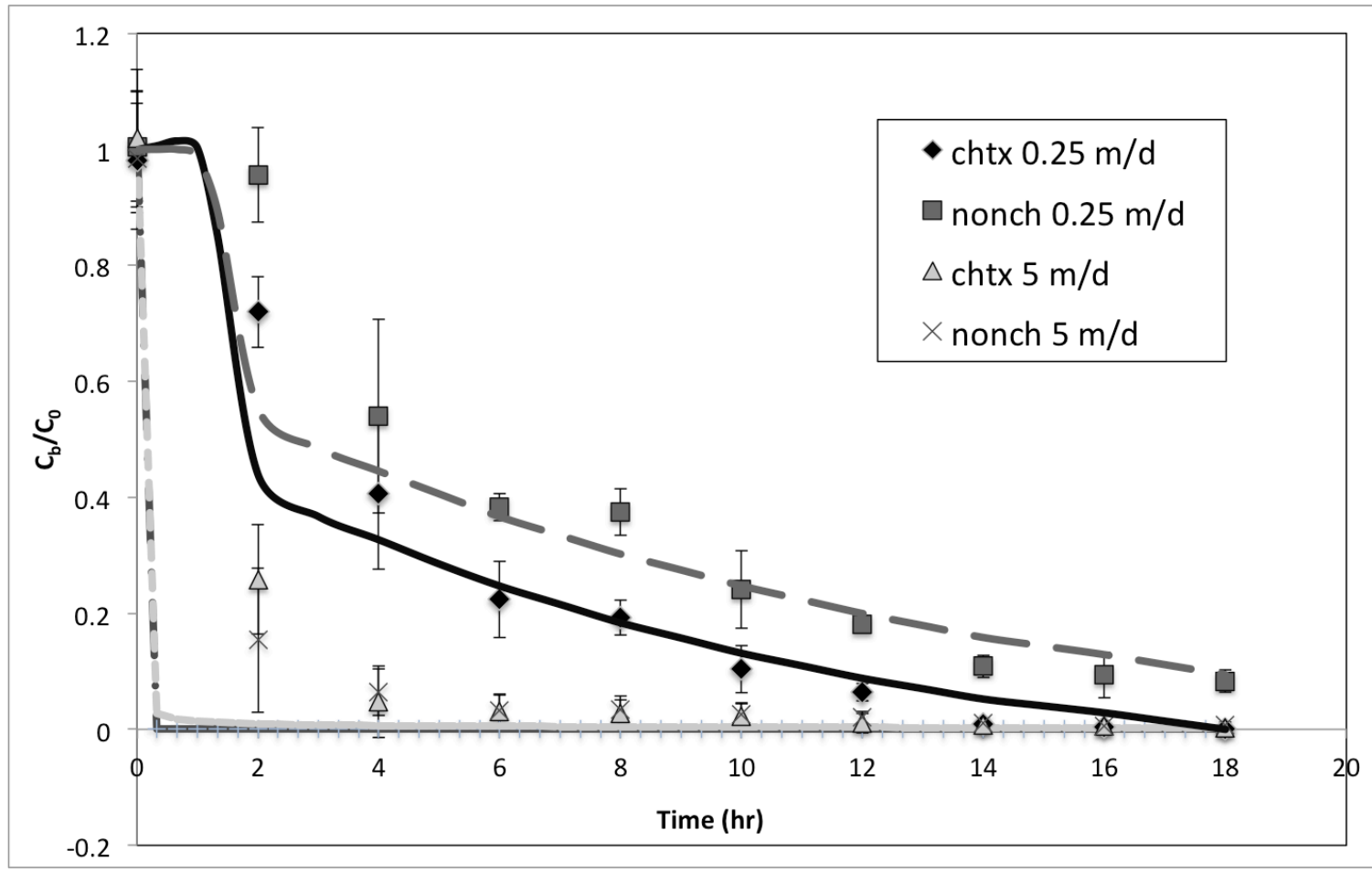

Figure 5.11 The breakthrough curves of chemotactic and nonchemotactic bacteria at different flow rates. "chtx" and "nonch" in the legend stand for chemotactic and nonchemotactic bacteria respectively. The lines are the fitting results corresponding to different experimental datasets (dataset number $\mathrm{n}=3$ ). The fittings were run at a time interval of $20 \mathrm{~min}$.

Another thing to notice is that the surface areas under the curves at $0.25 \mathrm{~m} / \mathrm{d}$ are not the same. This difference in area is related to the washed-out bacterial population during the 18 hours, as $B_{\text {total }}=B_{\text {wash }}(t)+B_{\text {in }}(t)$, where $B$ means the normalized bacterial population, and $\mathrm{B}_{\text {total }}$ is $100 \%$ and the same for both chemotactic and nonchemotactic bacteria. $\mathrm{B}_{\text {wash }}$ stands for the bacterial population that is washed out and is the surface area under the curve, and $\mathrm{B}_{\text {in }}$ is the bacterial population remained in the device. Both 
$B_{\text {wash }}$ and $B_{\text {in }}$ change with time (t), and $B_{\text {wash }}$ increases with time because more and more bacteria are washed out. The area for chemotactic is smaller than that of nonchemotactic after 18 hours, and this means that less chemotactic bacteria are washed out, and thus indicates more chemotactic bacteria remain in the device due to chemotaxis effect in presence of attractant gradient. And because the flow rate is very slow ( $0.25 \mathrm{~m} /$ day), so the chemotactic bacteria in the device may be not able to be washed out at all. In addition, chemotactic bacteria were not totally washed out after 18 hours, and bacterial density did not drop to zero at the end of the curve; the value of the bacterial density at 18 hours was close to 0.01 , which was hard to distinguish from zero in the figure, and neither did the fitting curve drop to zero.

If the flow rate increased to $5 \mathrm{~m} / \mathrm{d}$, both chemotactic and nonchemotactic bacteria were washed out very quickly, as both bacteria densities dropped to the level close to zero just after several minutes of injection, as shown in Figure 5.11. These breakthrough curves at high flow rate were significant different from the trends at low flow rate from a statistical viewpoint with $\mathrm{p}<0.0001$. However, at a flow rate of $5 \mathrm{~m} / \mathrm{d}$, the trend for chemotactic bacteria almost overlapped with that for nonchemotactic control, which means that the effect of chemotaxis was dominated by the large convection term. The dominance of high convective flow over chemotaxis effect was also found and supported by the results from Wang et al. (2012), in which high flow rates eliminated transverse bacterial accumulation due to chemotaxis. If we compare the bacterial swimming speed $v_{b}(44 \mu \mathrm{m} / \mathrm{s})$ with the flow rate, we notice that chemotaxis happens when $v_{b}$ is larger than the flow rate $(0.25 \mathrm{~m} / \mathrm{d}=2.9 \mu \mathrm{m} / \mathrm{s})$, and the phenomenon diminishes when $v_{b}$ is smaller than the flow rate $(5 \mathrm{~m} / \mathrm{d}=58 \mu \mathrm{m} / \mathrm{s})$, which is consistent with the previous study of $P$. 
putida bacteria and toluene in the single-pore device (Wang et al., 2012). Because the microfluidic device mimics the essential features (pore sizes, contaminant profile and heterogeneity etc.) of in-situ NAPL contaminated sites, it may provide a good indication of how bacterial chemotaxis will influence bacterial distributions in natural aquifers, and also provide evidence that chemotactic bacteria accumulate near contaminant sources in the low permeable region under certain groundwater flow rates. The breakthrough curve for chemotaxis differentiates from the one for nonchemotaxis, when groundwater flow rates are generally slow $(0.1-1 \mathrm{~m} / \mathrm{d})$, but the difference diminishes at a higher flow rate. This information can guide us to build up correct strategies to apply chemotaxis in fieldscale studies. According to this information, chemotaxis can be tested and possibly accelerate bioremediation in the contaminated sandy aquifer at Cape Cod, MA, as the sites have similar porous structure and groundwater velocity from 0.25 to $0.33 \mathrm{~m} / \mathrm{d}$ (Harvey and Garabedian, 1991); however, chemotaxis would not make a difference if the flow rates are much higher than that. The experimental results for $5 \mathrm{~m} / \mathrm{d}$ were also supported by the fitted curves in Figure 5.11, as both chemotactic and nonchemotactic bacteria subjected to a quick washout under bulk flow at $5 \mathrm{~m} / \mathrm{d}$. However, the fitting did not match well with the data points at two hours of injection; the experimental observation showed a higher density of both bacterial strains than the fitted results at either flow rate. From the aspect of experiment, time zero of the injection was set to be the time when the injectate changed from bacterial suspension to washout buffer at the inlet, so the injectate change might generate the delay of washout due to various of reasons about the device and the operation. From the aspect of fitting, different values of permeability $\kappa_{b r}$ could affect the breakthrough trends to some extent at the slow flow rate, 
and an accurate estimation of this value needed to be developed in future, in order to obtain more accurate fittings. Currently, $\kappa_{b r}$ is the only fitting parameter, while all other parameters are either calculated in this study or reported by literatures.

\subsection{Implications}

The chemotaxis effect is proved to efficiently deliver more degrading bacteria to the NAPL contaminant sources in low permeable region, based on the data from the continuous injection, no flow and buffer wash experiments in this chapter. The results are also supported by mathematical models and statistical analysis. There is no direct observation of biodegradation, but because many NAPL contaminants such as toluene are degradable by $P$. putida F1, the experimental observations of biased accumulation of chemotactic bacteria around the NAPL sources within the low permeable region will lead to an increase of contaminant consumption, and because toluene is also one of the carbon sources for $P$. putida strains, a faster growth of overall bacterial population is expect to happen, which will further accelerate the biodegradation to a greater extent. Therefore, bacterial chemotaxis reveals a large potential to improve the efficiency of bioremediation. However, groundwater flow rate is an important factor to consider before applying chemotactic bacteria to contaminated sites. In general, a local flow rate smaller than bacterial swimming speed may allow sufficient retention time for chemotactic bacteria to swim toward the contaminant sources in the transverse direction; however, a higher flow rate overwhelms the chemotaxis effect and carries bacteria away along the groundwater flow pathway. 


\section{SUMMARY AND CONCLUSIONS}

\section{Chapter 6}

\section{SUMMARY AND}

\section{Cconclusions}

\subsection{Summary}

Two different microfluidic devices were designed and fabricated to study bacterial chemotaxis. Convection free microfluidic device ( $\mathrm{CF}-\mu \mathrm{Chip})$ successfully extremely simplified the experimental condition to measure the two essential chemotaxis parameters: chemotactic sensitivity coefficient $\chi_{0}$ and chemotactic receptor constant $K_{c}$. CF- $\mu$ Chip provided an accurate method to measure these parameters, and decoupled the compensating effect between $\chi_{0}$ and $K_{c}$, which was important to solve but neglected in previous studies, by testing bacterial response to a different attractant concentration. The 
device material was changed from glass to an air permeable polymer (PDMS), which could keep the oxygen level in the device so that aerobic bacteria remained motile and also allowed a simple and reversible assembly of the device. Both Pseudomonas putida and Escherichia coli strains were studied with corresponding attractant and labeled with different fluorescence dyes, and the bacterial densities were recorded as fluorescence light intensities from microscope linked camera. More chemotactic population migrated into the channel at appropriate attractant concentration than nonchemotactic bacteria, and the predicted chemotaxis parameters were consistent with the published results and the values calculated from related work.

The other microfluidic device (H- $\mu$ Chip) was designed to mimic the heterogeneous groundwater system that was exposed to NAPL contaminant sources. The degrading bacteria were injected in the high permeable region with convective flow within the typical range of groundwater flow rates, and the NAPL contaminants were trapped at designated locations in the low permeable region. Pseudomonas putida F1 and toluene were used as the test models for chemotactic bacteria and attractant. Bacterial distributions were compared between the wild type and the nonchemotactic mutant in three different experimental conditions: intersection between horizontal channel and porous network with continuous injection of bacteria, adjacent area outside each toluene ganglion with no flow, and the device outlet with continuous buffer wash. Evidences of chemotaxis were found for all different conditions by comparing its effect with nonchemotactic bacterial distribution. The impact of flow rate on chemotaxis was also studied in the two with-flow conditions. 
Mathematical models were solved numerically in a finite element solver COMSOL and the fitted results supported the experimental findings throughout this study. Statistical analysis was also widely applied to the quantitative comparisons of experiments.

\subsection{Conclusions}

Fluorescence labeling was successfully used to detect bacteria. The current fluorescence method created a sufficient signal-to-noise ratio so that the fluorescence intensity could directly indicate the bacterial density, and this method is more accurate and takes much less time to analyze than the conventional methods, such as counting bacteria either under the microscope or on the computer screen. Furthermore, chemotactic and nonchemotactic bacteria were mixed and injected together into the devices, and this reduced many possible operational and systematic errors from experiment to experiment.

The material of CF- $\mu$ Chip was changed from glass to PDMS, and the latter ensured sufficient oxygen accessibility to keep aerobic bacteria staying healthy and motile in the device.

The fitting results from $\mathrm{CF}-\mu \mathrm{Chip}$ indicated that the averaged value of chemotactic sensitivity coefficient $\chi_{0}$ is $1.8 \times 10^{-4} \mathrm{~cm}^{2} / \mathrm{s}$, and chemotactic receptor constant $K_{c}$ is $1 \mathrm{mM}$ for $P$. putida $\mathrm{F} 1$ to toluene, which are similar to the reported values for $P$. putida F1/TCE (Olson et al., 2004). Modeling fitting to experimental data of $E$. coli HCB1 with $\alpha$-methylaspartate ( $\alpha$-mASP) showed that $\chi_{0}$ is $2.4 \times 10^{-4} \mathrm{~cm}^{2} / \mathrm{s}$ and $K_{c}$ is 0.13 $\mathrm{mM}$, both supported by previous reference (Lewus and Ford, 2001). 
When groundwater flow rate was small in H- $\mu$ Chip, chemotactic bacteria formed a biased transverse accumulation toward the NAPL contaminant sources in the low permeable porous network; however, such biased movement did not occur for nonchemotactic bacteria. Thus, chemotactic bacteria showed the ability to direct more cellular population into the contaminated region with fine pore sizes.

At the slow groundwater flow rate in $\mathrm{H}-\mu \mathrm{Chip}$, chemotactic bacteria was more difficult to be washed out from the device, because the chemotaxis effect induced by the attractant gradient in the porous network generated a transverse migration of bacteria to confront the major bulk flow in the horizontal channel. This longer retention time of chemotactic bacteria in the contaminated porous network increased the opportunity of contaminant consumption.

More direct evidence of chemotaxis was found in vicinity of toluene ganglia in $\mathrm{H}$ $\mu$ Chip when the flow stopped. Chemotactic bacteria exhibited a $15 \%$ more accumulation near one contaminant source than the ones located $500 \mu \mathrm{m}$ away from the ganglion, while nonchemotactic bacteria followed an even distribution. The increase level and the location of accumulation were consistent with previous results about chemotactic accumulation near a toluene/water interface within a single pore interstice (Wang et al., 2012).

Flow rate played a major role in affecting chemotaxis effect in porous media. A rough estimation showed that if the flow rate was smaller than the bacterial swimming speed, then it left sufficient time for bacteria to interact with the attractant gradient on the transverse direction and redirect their movement, but if the flow rate was higher, the retention time for bacteria to interact with chemical change in the surrounding was fairly 
small, majority of the bacterial population would be carried away by the dominate convective flow along the water pathway.

Numerical solutions solved from the mathematical models also matched the experimental results in this study, and the fitting parameters are listed in Table 6.1. $\chi_{0}$ and $K_{c}$ were calculated based on the experimental data from CF- $\mu$ Chip.

Table 6.1 Main parameters for the computational fitting of bacterial chemotaxis

\begin{tabular}{lcc}
\hline & P. putida $\mathrm{F} 1$ and F1 CheA & E. coli $\mathrm{HCB} 1$ and HCB437 \\
\hline$T[\mathrm{~K}]$ & 298 & 298 \\
$\chi_{0}\left[\times 10^{-8} \mathrm{~m}^{2} / \mathrm{s}\right]$ & 1.8 & 2.4 \\
$K_{c}[\mathrm{mM}]$ & 1.0 & 0.125 \\
$b_{0}\left[\mathrm{~mol} / \mathrm{m}^{3}\right]$ & 1.0 & 1.0 \\
$v_{b}[\mu \mathrm{m} / \mathrm{s}]$ & 44 & 22.8 \\
$D_{a}\left[\times 10^{-10} \mathrm{~m}^{2} / \mathrm{s}\right]$ & $9.5($ toluene $)$ & $8.7(\alpha-\mathrm{mASP})$ \\
$\mu_{0}\left[\times 10^{-10} \mathrm{~m}^{2} / \mathrm{s}\right]$ & 13 & 2.4 and 17 \\
$\kappa_{b r}\left[\times 10^{-9} \mathrm{~m}^{2}\right]$ & 1.0 & N/A \\
$\varepsilon_{p}$ & 0.43 & N/A \\
\hline
\end{tabular}

\subsection{Recommendations}

$\mathrm{CF}-\mu \mathrm{Chip}$ is an accurate and convenient tool to measure chemotaxis parameters, and should be widely used for different bacteria and chemical stimulus combinations, in 
order to enrich the poorly quantitative database for bacterial chemotaxis. Multiple attractants/repellents can also be added into the channel at the same time to study chemotaxis in a more complex chemical environment. However, in order to capture more accurate values of chemotaxis parameters, sensitivity of fluorescence detection needs to be enhanced and background noise, such as unstable light source from arc lamp, needs to be reduced. Bacterial distribution in presence of a wider range of attractant concentration is recommended to test in order to find out if $K_{c, \text { low }}$ exists for certain bacterial strains.

Furthermore, NAPL biodegradation should be studied in the H- $\mu$ Chip, and the biodegradation process can be recorded either as the decrease in NAPL concentration at the outlet or the increase of bacterial population in the device on a time scale. 


\section{REFERENCES}

1. Ahmed and Stocker. Experimental verification of the behavioral foundation of bacterial transport parameters using microfluidics. Biophys J (2008) 95(9): 4481-93

2. Alagappan, Gunaseelan, and Robert M. Cowan. Effect of temperature and dissolved oxygen on the growth kinetics of Pseudomonas putida F1 growing on benzene and toluene. Chemosphere 54, no. 8 (2004): 1255-1265.

3. Alexander. Biodegradation and bioremediation. Academic Press, San Diego (1994)

4. Atencia and Locascio. Chips \& Tips: Simple and reversible bonding of glass to glass, quartz and sapphire wafers. Lab on a Chip (2009). RSC Publishing Blogs.

5. Atencia et al. A robust diffusion-based gradient generator for dynamic cell assays. Lab Chip (2012) vol. 12, 309-316

6. Atlas and Bartha. Microbial Ecology: Fundamentals and Applications $4^{\text {th }}$ Ed. Dorling Kindersley (India) Pvt. Ltd. (2007) pp. 576 ISBN 81-317-1384-9

7. Atlas and Pramer. Focus on bioremediation. ASM News (1990) vol. 56 pp. 7

8. Auset and Keller. Pore-scale processes that control dispersion of colloids in saturated porous media. Water Resour Res (2004) 40 (3) W03503

9. Auset and Keller. Visualization of colloidal mobility and removal at the pore scale in soil saturated conditions. Geophysical Research Abstracts (2006) 8: 10221

10. Bauer et al. Mixing-controlled biodegradation in a toluene plume-results from twodimensional laboratory experiments. J Contaminant Hydrology (2008) vol. 96 pp. 150-168

11. Bauer et al. Enhanced biodegradation by hydraulic heterogeneities in petroleum hydrocarbon plumes. J Contaminant Hydrology (2009) vol. 105 pp. 56-68 
12. Berg and Brown. Chemotaxis in Escherichia coli analyzed by 3-dimensional tracking. Nature (1972) vol. 239 (5374) pp. 500

13. Berg and Turner. Chemotaxis of bacteria in glass capillary arrays. Escherichia coli, motility, microchannel plate, and light scattering. Biophysical J (1990) vol. 58 pp. 919-930

14. Bibikov et al. A signal transducer for aerotaxis in Escherichia coli. J Bacteriol. (1997) vol. 179 pp. 4075-79

15. Boopathy. Factors limiting bioremediation technologies. Bioresource Technol (2000) vol. 74 pp. 63-67

16. Brashear. B.S. thesis, 2009, University of Virginia

17. Bray and Lay. Computer-simulated evolution of a network of cell-signaling molecules. Biophys J (1994) vol. 66 (4) pp. 972-977

18. Burke. Solubility Parameters: Theory and Application. The AIC Book and Paper Group Annual (1984) vol. 3, Craig Jensen, Editor, pp. 13-58

19. Chandak et al. Sorption and diffusion of VOCs in DAY zeolite and silicalite-filled PDMS membranes. J Membrane Sci (1997) vol. 133 pp. 231-243

20. Chen and Ford. Perturbation expansion of Alt's cell balance equations reduces to Segel's 1D equations for shallow chemoattractant gradients. SIAM J Appl Math (1998) vol. 59 pp. 35-57

21. Chen et al. Mathematical models for motile bacterial transport in cylindrical tubes. J. Theoretical Biology (1998) vol. 195 pp. 481-504 
22. Chen et al. Stochastic analysis of transient three-phase flow in heterogeneous porous media. Stochastic Environmental Research and Risk Assessment (2009) vol. 23 (1) pp. 93-109

23. Cheng et al. A hydrogel-based microfluidic device for the studies of directed cell migration. Lab on a Chip (2007) vol. 7 (6) pp. 763-769

24. Clarke and Koshland. Membrane receptors for aspartate and serine in bacterial chemotaxis. J Biological Chemistry (1979) vol. 254 pp. $9695-9702$

25. Crist et al. Pore-scale visualization of colloid transport and retention in partly saturated porous media. Vadose Zone Journal (2004) vol. 3(2) pp. 444-450

26. Dahlquist, F. W., P. Lovely, and D. E. Koshland. Quantitative analysis of bacterial migration in chemotaxis. Nature 236, no. 65 (1972): 120-123.

27. DiLuzio et al. Escherichia coli swim on the right-hand side. Nature (2005) vol. 435 (7046) pp. 1271-1274

28. Duffy and Ford. Turn angle and run time distributions characterize swimming behavior for Pseudomonas putida. J Bacteriol (1997) vol. 179 pp. 1428-1430

29. EPA542-R-08-002. Green remediation: incorporating sustainable environmental practices into remediation of contaminated sites. U. S. Environmental Protection Agency (2008)

30. Farhadian et al. In situ bioremediation of monoaromatic pollutants in groundwater: A review. Bioresource Technol (2008) vol. 99(13) pp. 5296-5308

31. Ford et al. Measurement of bacterial random motility and chemotaxis coefficients: I. Stopped-flow diffusion chamber assay. Biotechnol. Bioeng. 37 (1991) 647-660 
32. Ford and Lauffenburger. Measurement of bacterial random motility and chemotaxis coefficients: II. Application of single-cell-based mathematical model. Biotechnol. Bioeng. 37 (1991) 661-672

33. Ford and Lauffenburger. Analysis of chemotactic bacterial distributions in population migration assays using a mathematical model applicable to steep or shallow attractant gradients. Bulletin of Mathematical Biology (1991) vol. 53 pp. 721-749

34. Ford and Harvey. Role of chemotaxis in the transport of bacteria through saturated porous media. Adv Water Resour (2007) vol. 30 pp. 1608-1617

35. Frymier et al. Cellular-dynamics simulations of bacterial chemotaxis. Chem Eng Sci (1993) vol. 48 (4) pp. 687-699

36. Frymier and Ford. Analysis of bacterial swimming speed approaching a solid-liquid interface. Aiche J (1997) vol. 43 (5) pp. 1341-1347

37. Garabedian, Stephen P., Dennis R. LeBlanc, Lynn W. Gelhar, and Michael A. Celia. Large-scale natural gradient tracer test in sand and gravel, Cape Cod, Massachusetts: 2. Analysis of spatial moments for a nonreactive tracer. Water Resources Research 27, no. 5 (1991): 911-924.

38. Gargiulo et al. Bacteria transport and deposition under Unsaturated Flow Conditions: The Role of Water Content and Bacteria Surface Hydrophobicity. Vadose Zone Journal (2008) vol. 7(2) pp. 406-419

39. Grebe and Stock. Bacterial chemotaxis: the five sensors of a bacterium. Current Biology (1998) vol. 8 pp. 75.98

40. Grimm and Harwood. Chemotaxis of Pseudomonas spp. to the polyaromatic hydrocarbon naphthalene. Appl Environ Microb (1997) vol. 63 (10) pp. 4111-4115 
41. Grimm and Harwood. NahY, a catabolic plasmid-encoded receptor required for chemotaxis of Pseudomonas putida to the aromatic hydrocarbon naphthalene. J Bacteriol. (1999) vol. 181(10):3310-6.

42. Grindstaff. Bioremediation of chlorinated solvent contaminated groundwater. U. S. EPA (1998)

43. Hanahan. Studies on transformation of Escherichia coli with plasmids. J Mol Biol. (1983) vol. 166(4):557-80

44. Harms and Wick. Dispersing pollutant-degrading bacteria in contaminated soil without touching it. Eng Life Sci (2006) vol. 6 (3) pp. 252-260

45. Hart. In situ bioremediation: Defining the limits. Environmental science \& technology (1996) vol. 30 pp. 398-401

46. Harter. Basic concepts of groundwater hydrology, Tech. Rep. Publ. 8083, Univ. of Calif. (2003)

47. Harvey, Ronald W., and Stephen P. Garabedian. Use of colloid filtration theory in modeling movement of bacteria through a contaminated sandy aquifer.

Environmental science \& technology 25.1 (1991): 178-185.

48. Harwood et al. Flagellation of pseudomonas-putida and analysis of its motile behavior. J Bacteriol (1989) vol. 171 (7) pp. 4063-4066

49. Harwood et al. Identification of the pcarkf gene-cluster from pseudomonas-putida involvement in chemotaxis, biodegradation, and transport of 4-hydroxybenzoate. $\mathrm{J}$ Bacteriol (1994) vol. 176 (21) pp. 6479-6488

50. Hassink et al. Relationships between habitable pore-scale, soil biota and mineralization rate in grassland soils. Soil Biol Biochem (1993) vol. 25 pp. 47-55 
51. Head. Bioremediation: towards a credible technology. Microbiol-Uk (1998) vol. 144 pp. $599-608$

52. Hillen and Painter. A user's guide to PDE models for chemotaxis. Journal of Mathematical Biology (2009)

53. Hoang et al. A broad-host-range Flp-FRT recombination system for site-specific excision of chromosomally-located DNA sequences: application for isolation of unmarked Pseudomonas aeruginosa mutants. Gene (1998) 212(1):77-86

54. Imae et al. Phenol - A complex chemoeffector in bacterial chemotaxis. J Bacteriol (1987) vol. 169 (1) pp. 371-379

55. Jasuja, Ravi, David R. Trentham, and Shahid Khan. Response tuning in bacterial chemotaxis. Proceedings of the National Academy of Sciences 96, no. 20 (1999): $11346-11351$.

56. Kamholz et al. Quantitative analysis of molecular interaction in a microfluidic channel: The T-sensor. Anal Chem (1999) vol. 71 (23) pp. 5340-5347

57. Kavanaugh et al. The DNAPL Remediation Challenge: Is There a Case for Source Depletion? EPA/600/R-03/143 (2003)

58. Keller and Segel. Model for chemotaxis. J Theor Biol (1971) vol. 30 pp. 225-34

59. Knutson et al. Pore-scale modeling of dissolution from variably distributed nonaqueous phase liquid blobs. Water Resour Res (2001) vol. 37 pp. 2951-2963

60. Kusy, K. PhD dissertation, 2005, University of Virginia

61. Kusy and Ford. Surface association of motile bacteria at granular porous media interfaces. Environ Sci Technol. (2009) vol. 43(10):3712-9 
62. Langwaldt and Puhakka. On-site biological remediation of contaminated groundwater: a review. Environ Pollut (2000) vol. 107 pp. 187-197

63. Lanning et al. Bacterial chemotaxis transverse to axial flow in a microfluidic channel. Biotechnol Bioeng (2008) 100 (4) 653-663

64. Lanning, L. M. PhD dissertation, 2004, University of Virginia

65. Law and Aitken. Bacterial chemotaxis to naphthalene desorbing from a nonaqueous liquid. Appl Environ Microb (2003) vol. 69 (10) pp. 5968-5973

66. Law and Aitken. Continuous-flow capillary assay for measuring bacterial chemotaxis. Appl Environ Microb (2005) vol. 71 pp. 3137-3143

67. Lederberg. Linear inheritance in transductional clones. Genetics (1956) vol. 41 pp. $845-871$

68. Lee, Jessamine Ng, Cheolmin Park, and George M. Whitesides. Solvent compatibility of poly (dimethylsiloxane)-based microfluidic devices. Analytical chemistry 75, no. 23 (2003): 6544-6554.

69. Lewus and Ford. Quantification of random motility and chemotaxis bacterial transport coefficients using individual-cell and population-scale assays. Biotechnol Bioeng (2001) vol. 75 pp. 292-304

70. Liu and Ford. Idling time of swimming bacteria near particulate surfaces contributes to apparent adsorption coefficients at the macroscopic scale under static conditions. Environmental science \& technology (2009) 43 (23), pp 8874-8880

71. Liu et al. TCE dechlorination rates, pathways, and efficiency of nanoscale iron particles with different properties. Environmental science \& technology (2005) vol. 39 pp. $1338-1345$ 
72. Liu et al. Chemotaxis to pyrimidines and identification of a cytosine chemoreceptor in Pseudomonas putida. J. Bacteriol. (2009) vol. 191 pp. 2909-16

73. Liu and Papadopoulos. Unidirectional motility of Escherichia coli in restrictive capillaries. Appl. Environ. Microbiol. (1995) vol. 61 pp. 3567-72

74. Long and Ford. Enhanced Transverse Migration of Bacteria by Chemotaxis in a Porous T-Sensor. Environmental science \& technology (2009) vol. 43 (5) pp. 15461552

75. Long and Hilpert. Lattice-Boltzmann modeling of contaminant degradation by chemotactic bacteria: Exploring the formation and movement of bacterial bands. Water Resour Res (2008) vol. 44(9) W09415

76. Lovely and Dahlquist. Statistical measures of bacterial motility and chemotaxis. J Theor Biol (1975) vol. 50 (2) pp. 477-496

77. Macnab and Koshland. Gradient-sensing mechanism in bacterial chemotaxis. P Natl Acad Sci USA (1972) vol. 69 (9) pp. 2509

78. Marx and Aitken. Bacterial chemotaxis enhances naphthalene degradation in a heterogeneous aqueous system. Environmental science \& technology (2000) vol. 34 (16) pp. 3379-3383

79. Marx and Aitken. Quantification of chemotaxis to naphthalene by Pseudomonas putida G7. Appl Environ Microb (1999) vol. 65 pp. 2847-2852

80. Miyagi et al. Tremor induced by toluene misuse successfully treated by a Vim thalamotomy. J Neurol Neurosurg Psychiatry (1999) 66: 794-796

81. Montgomery. Groundwater Chemicals Desk Reference $2^{\text {th }}$ Ed. Lewis Publishers (1996) 
82. Newell et al. Impact of non-aqueous phase liquids (NAPLs) on groundwater remediation. ALCHE, Symposium 23, 1994

83. Newell et al. Ground Water Issue: Light Nonaqueous Phase Liquids. EPA/540/S$95 / 500(1995)$

84. Olson et al. Mathematical modeling of chemotactic bacterial transport through a twodimensional heterogeneous porous medium. Bioremediation Journal (2006) vol. 10(12) pp. 13-23

85. Olson et al. Quantification of Bacterial Chemotaxis in Porous Media Using Magnetic Resonance Imaging. Environmental science \& technology (2004) vol. 38 pp. 38643870

86. Olson, M. M. PhD dissertation, 2004, University of Virginia

87. O'Toole and Kolter. Flagellar and twitching motility are necessary for Pseudomonas aeruginosa biofilm development. Molecular Microbiology (1998) vol. 30(2) pp. 295304

88. Pandey and Jain. Bacterial chemotaxis toward environmental pollutants: Role in bioremediation. Appl Environ Microb (2002) vol. 68 pp. 5789-5795

89. Parales et al. Toluene-degrading bacteria are chemotactic towards the environmental pollutants benzene, toluene, and trichloroethylene. Appl Environ Microb (2000) vol. 66 pp. $4098-4104$

90. Pedit et al. Quantitative analysis of experiments on bacterial chemotaxis to naphthalene. Biotechnol Bioeng (2002) vol. 78 pp. 626-634

91. Poole. Microbes on the move. New Scientist (1990) 3 March pp. 38-41 
92. Powelson and Mills. Transport of Escherichia coli in sand columns with constant and changing water contents. Am Soc Agronom (2001) vol. 30(1) pp. 238-245

93. Pratt and Kolter. Genetic analysis of Escherichia coli biofilm formation: roles of flagella, motility, chemotaxis and type I pili. Molecular Microbiology (1998) vol. 30(2) pp. 285-293

94. Prigent-Combaret et al. Abiotic surface sensing and biofilm-dependent regulation of gene expression in Escherichia coli. J Bacteriol (1999) vol. 181(19) pp. 5993-6002

95. Rani et al. Rapid diffusion of fluorescent tracers into staphylococcus epidermidis biofilms visualized by time lapse microscopy. Antimicrob. Agents Chemother. (2005) vol. 49(2): pp. 728-732

96. Rebbapragada et al. The Aer protein and the serine chemoreceptor Tsr independently sense intracellular energy levels and transduce oxygen, redox, and energy signals for Escherichia coli behavior. PNAS (1997) vol. 94 pp. 10541-6

97. Roper et al. Microfluidic Chip for Continuous Monitoring of Hormone Secretion from Live Cells Using an Electrophoresis-Based Immunoassay. American Chemical Society (2003) vol. 75 pp. $4711-4717$

98. Scow and Hicks. Natural attenuation and enhanced bioremediation of organic contaminants in groundwater. Curr Opin Biotech (2005) vol. 16 (3) pp. 246-253

99. Sherwood et al. Analysis of bacterial random motility in a porous medium using magnetic resonance imaging and immunomagnetic labeling. Environ. Sci. Technol. (2003) vol. 37 pp. $781-5$ 
100. Sirivithayapakorn and Keller. Transport of colloids in unsaturated porous media: a pore-scale observation of processes during the dissolution of air-water interface. Water Resour Res (2003) vol. 39(12) pp. 1346

101. Soby and Bergman. Motility and chemotaxis of rhizobium-meliloti in soil. Appl Environ Microb (1983) vol. 46 (5) pp. 995-998

102. Soga et al. A review of NAPL source zone remediation efficiency and the mass flux approach. J Hazard Mater (2004) vol. 110 (1-3) pp. 13-27

103. Sood. Chemotactic response of plant-growth-promoting bacteria towards roots of vesicular-arbuscular mycorrhizal tomato plants. Fems Microbiol Ecol (2003) vol. 45 (3) pp. 219-227

104. Stocker et al. Rapid chemotactic response enables marine bacteria to exploit ephemeral microscale nutrient patches. Proceedings of the National Academy of Sciences (2008) vol. 105(11) pp. 4209-4214

105. Sun and Chen. Sorption/desorption properties of ethanol, toluene, and xylene in poly (dimethylsiloxane) membranes. Journal of Applied Polymer Science (1994) vol. 51(10) pp. 1797-1804

106. Toepfer et al. Impact of fluorochrome stains used to study bacterial transport in shallow aquifers on motility and chemotaxis of Pseudomonas species. FEMS Microbiology Ecology (2012) vol. 81 pp. 163-171

107. Turner et al. Real-time imaging of fluorescent flagellar filaments. J Bacteriol (2000) vol. 182 (10) pp. 2793-2801

108. Urban et al., 485 Neurological and neurophysiological follow-up on workers with severe chronic exposure to toluene. Toxicology Letters (2003) vol. 144 pp. s130 
109. Wan and Wilson. Colloid transport in unsaturated porous media. Water Resour Res (1994a) vol. 30 pp. 857-864

110. Wan and Wilson. Visualization of the role of the gas-water interface on the fate and transport of colloids in porous-media. Water Resour Res (1994b) vol. 30 pp. 1123

111. Wan et al. Influence of the gas-water interface on transport of microorganisms through unsaturated porous media. Appl Environ Microb (1994) vol. 60(2) pp. 509516

112. Wang et al. Coupled effect of chemotaxis and growth on microbial distributions in organic-amended aquifer sediments: Observations from laboratory and field studies. Environmental science \& technology (2008) vol. 42 pp. 3556-3562

113. Wang and Ford. Transverse Bacterial Migration Induced by Chemotaxis in a Packed Column with Structured Physical Heterogeneity. Environmental science \& technology (2009) vol. 43 (15) pp. 5921-5927

114. Wang et al. 2012. Bacterial chemotaxis toward a NAPL source within a porescale microfluidic chamber. Biotechnology and Bioengineering 109.7: 1622-1628.

115. Weigl et al. Diffusion-based optical chemical detection in silicon flow structure. Analytical Methods and Instrumentation, MicroTAS 96 special edition

116. Whitesides. The origins and the future of microfluidics. Nature (2006) vol. 442 (7101) pp. 368-373

117. Wolfe et al. Reconstitution of signaling in bacterial chemotaxis. J. Bacteriol. (1987) vol. 169 pp. $1878-1885$ 
118. Yu and Alam. An agarose-in-plug bridge method to study chemotaxis in the Archaeon Halobacterium salinarum. FEMS Microbiology Letters (1997) vol. 156 pp. 265-269 


\section{APPENDIX A: COMSOL SETTINGS}

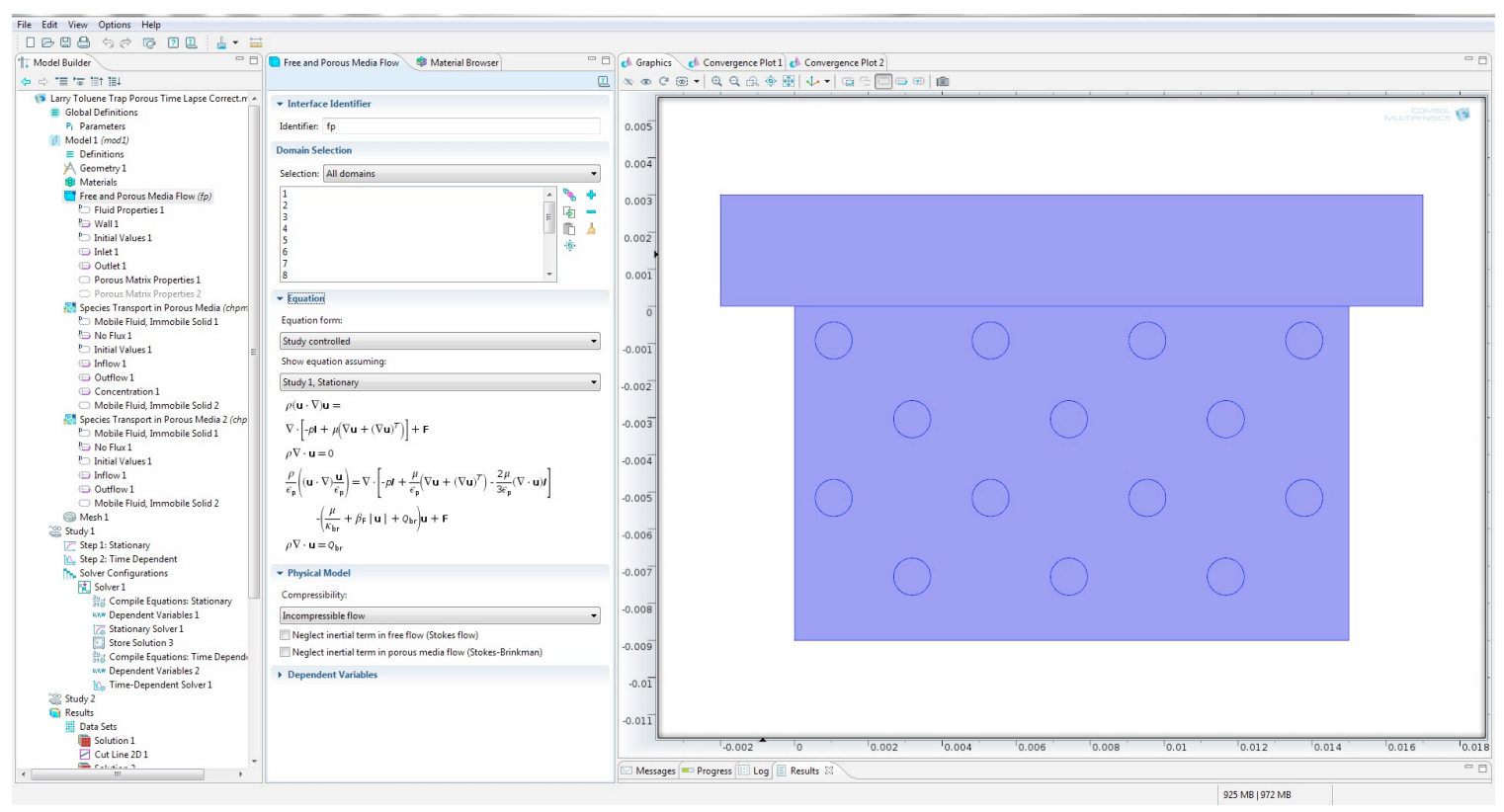

Figure A.1 General settings for the solvers of the modules in COMSOL.

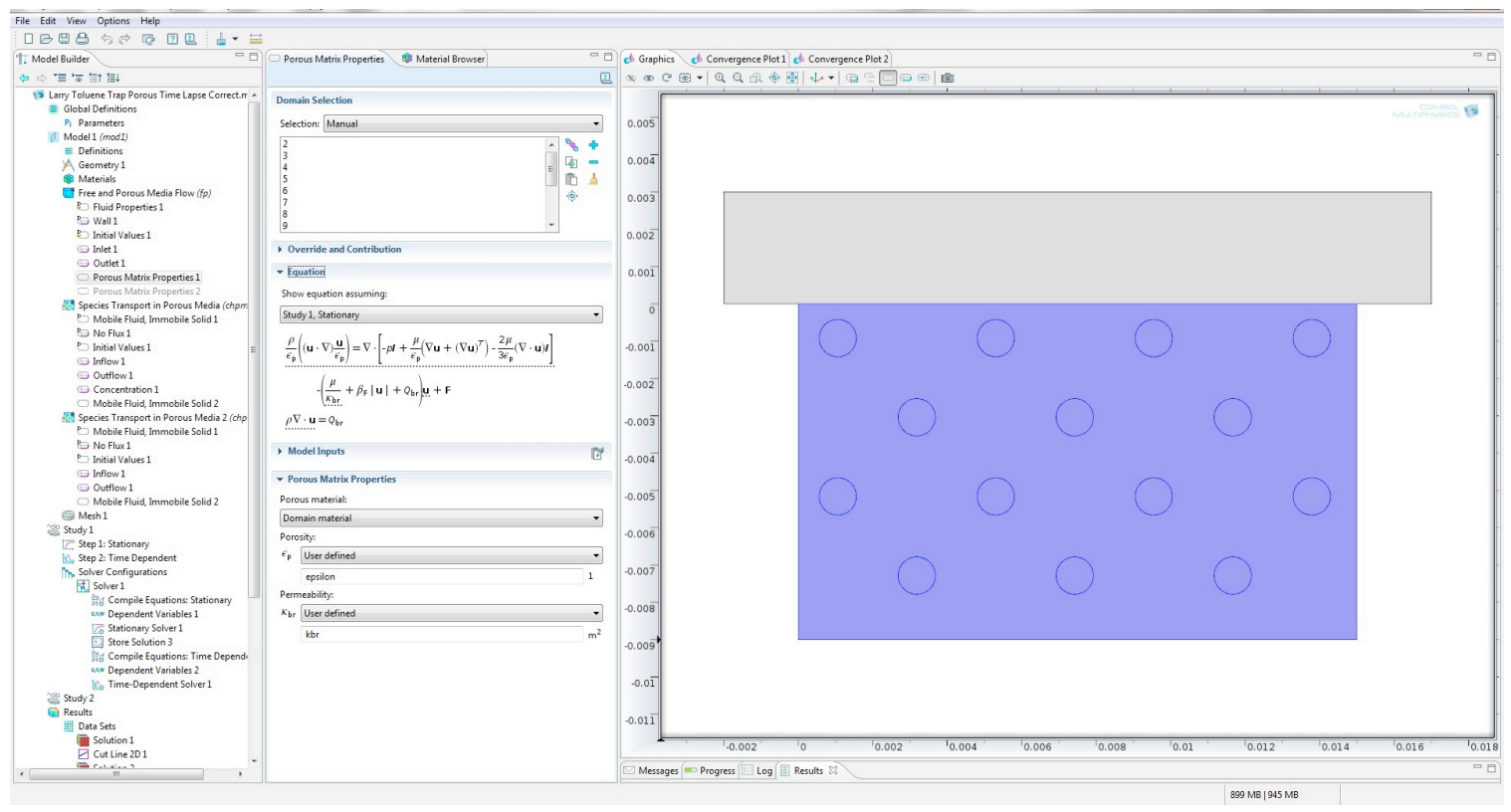

Figure A.2 General settings for the solvers of the modules in COMSOL. 


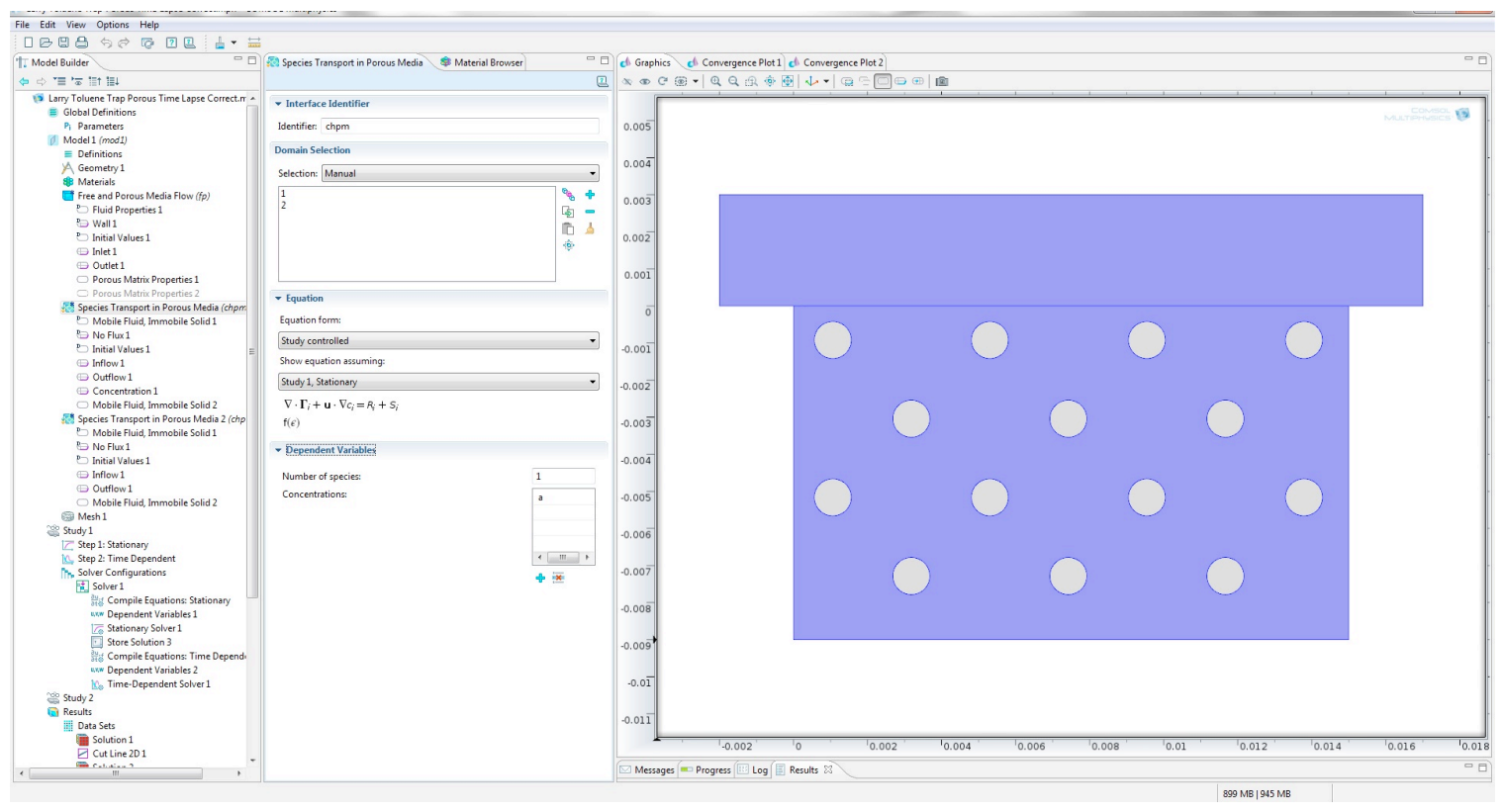

Figure A.3 General settings for the solvers of the modules in COMSOL.

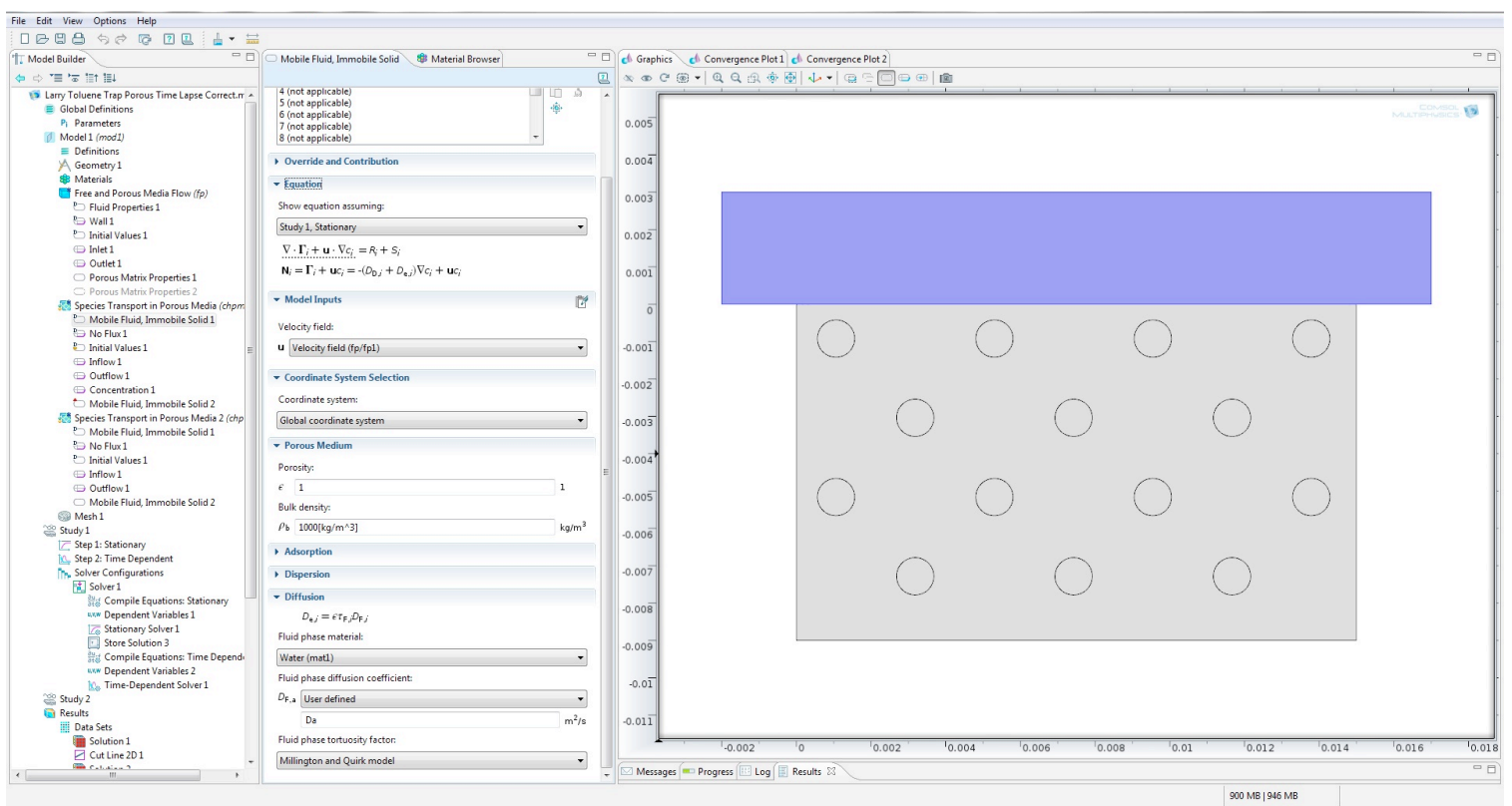

Figure A.4 General settings for the solvers of the modules in COMSOL. 


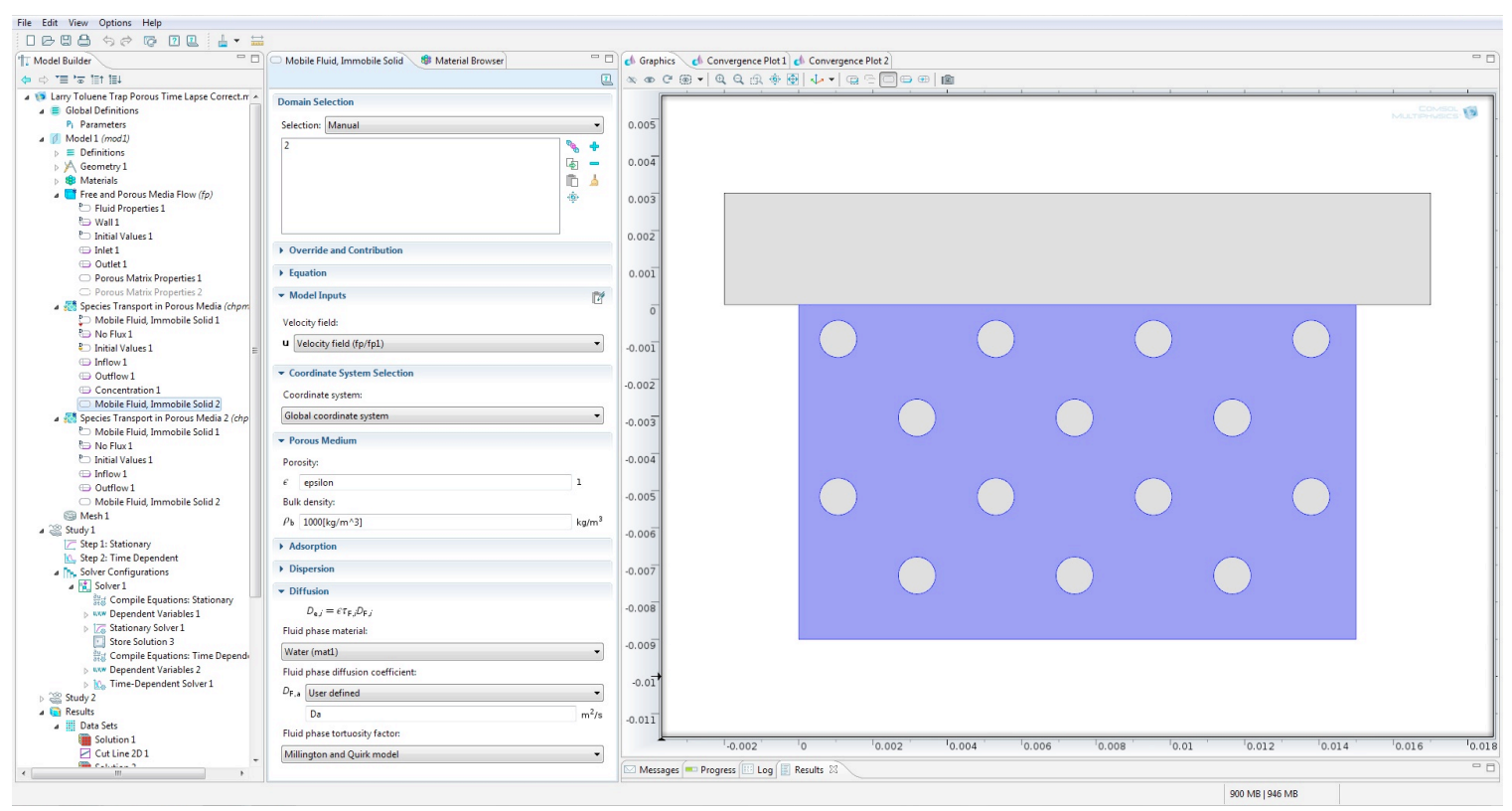

Figure A.5 General settings for the solvers of the modules in COMSOL.

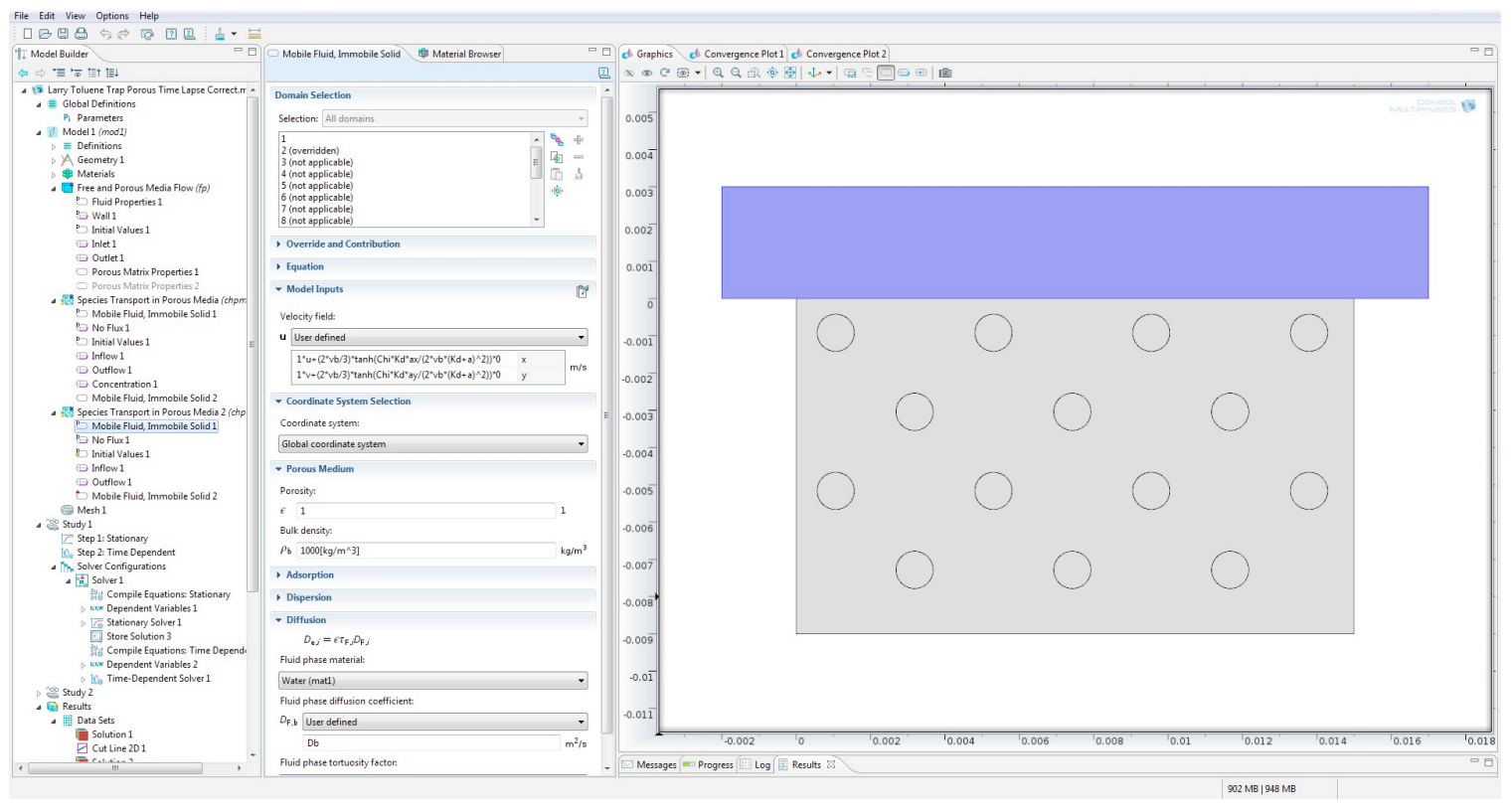

Figure A.6 General settings for the solvers of the modules in COMSOL. 


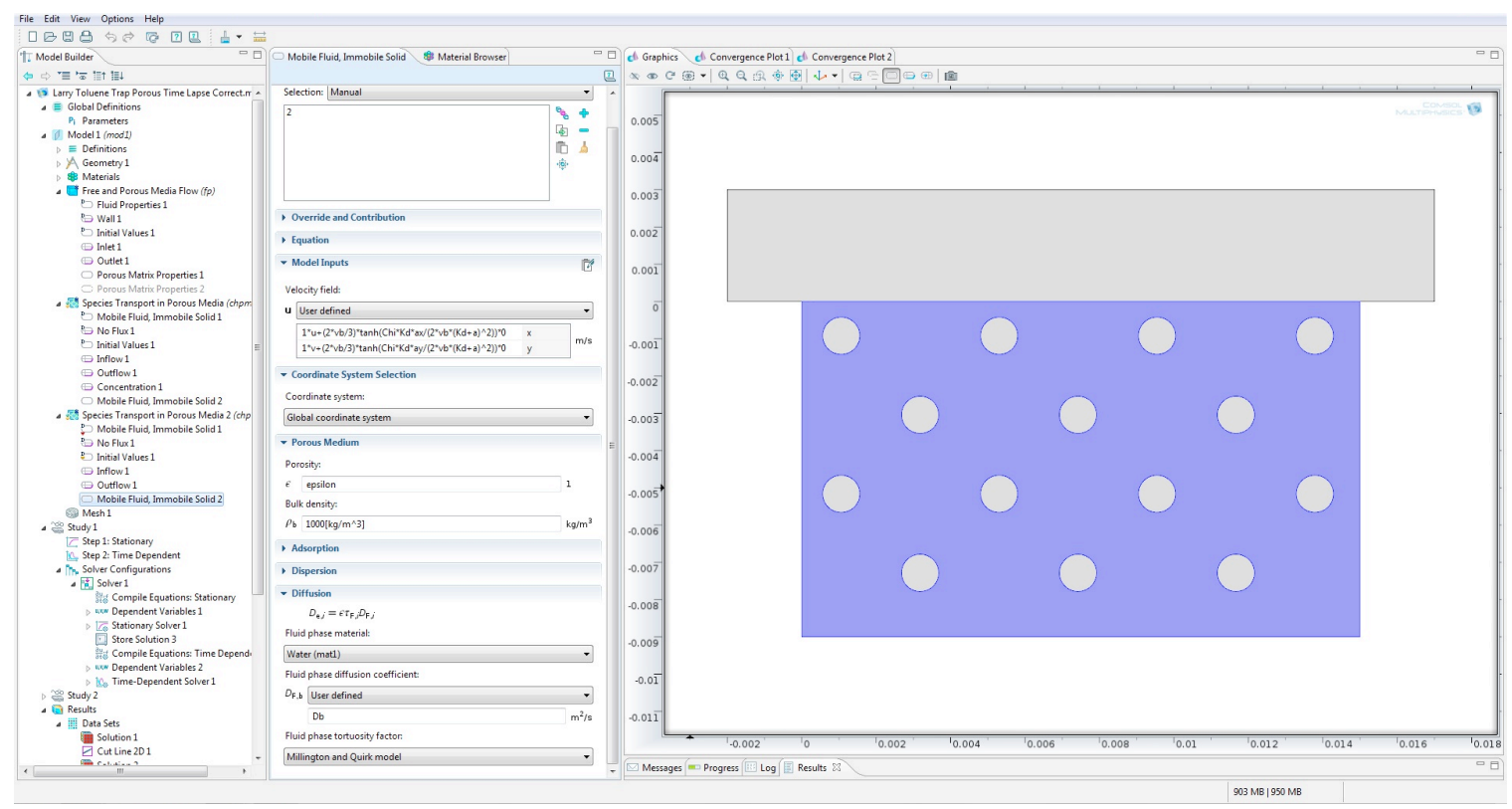

Figure A.7 General settings for the solvers of the modules in COMSOL. 


\section{APPENDIX B: TWO-WAY ANOVA ANALYSIS IN CF- $\mu$ CHIP}

Table B.1 Statistical comparison between the distributions of P. putida F1 at $2 \mathrm{mM}$ toluene and $0.2 \mathrm{mM}$ toluene in the $\mathrm{CF}-\mu \mathrm{Chip}$, as illustrated in Figure 4.6.

Table Analyzed

Data 1

Two-way ANOVA

Source of Variation

$\begin{array}{rr}\% \text { of total variation } & \text { P value } \\ 1.38 & <0.0001 \\ 2.27 & <0.0001 \\ 94.81 & <0.0001\end{array}$

Column factor

$94.81<0.0001$

Source of Variation

Interaction

Column factor

Row factor

$\begin{array}{rr}\text { P value summary } & \text { Significant? } \\ * * * & \text { Yes } \\ * * * & \text { Yes } \\ * * * & \text { Yes }\end{array}$

Source of Variation

Df Sum-of-squares

Interaction

Column factor

22

1843

Mean square

1

83.79

3039

3039

3.733

Row factor

22

126900

5769

Residual

92

2065

22.44

Number of missing values

0

Data analyzed: Data 1

\begin{tabular}{|c|c|c|}
\hline Source of Variation & Degrees of Freedom & $\underline{\text { Sum of Squares }}$ \\
\hline & Mean square & \\
\hline Column factor & 1.0 & 3039 \\
\hline Row factor & 22.0 & 126900 \\
\hline \multirow[t]{2}{*}{ Interaction } & 22.0 & 1843 \\
\hline & 83.79 & \\
\hline \multirow[t]{2}{*}{ Residual (error) } & 92.0 & 2065 \\
\hline & 22.44 & \\
\hline Total & 137.0 & 133900 \\
\hline
\end{tabular}

3039

5769 
Table B.2 Statistical comparison between the distributions of P. putida F1 at $2 \mathrm{mM}$ toluene and nonchemotactic $P$. putida $\mathrm{F} 1 \mathrm{CheA}$ in the $\mathrm{CF}-\mu \mathrm{Chip}$, as illustrated in Figure 4.6.

Table Analyzed Data 1

Two-way ANOVA

Source of Variation $\%$ of total variation $P$ value Interaction $3.04<0.0001$ Column factor $7.47<0.0001$

Row factor $88.50<0.0001$

Source of Variation Interaction Column factor

Row factor

Source of Variation

Interaction

Column factor

Row factor

Residual

$\begin{array}{rr}\text { P value summary } & \text { Significant? } \\ * * * & \text { Yes } \\ * * * & \text { Yes } \\ * * * & \text { Yes }\end{array}$

Number of missing values

Df Sum-of-squares

$15 \quad 0.3204$

Mean square

0.7872

0.7872

484.3

64

9.328

0.6218

382.6

Data analyzed: Data 1

\begin{tabular}{lll} 
Source of Variation & & \multicolumn{2}{l}{ Degrees of Freedom } & & Sum of Squares \\
Column factor & $\frac{\text { Mean square }}{1.0}$ & \\
& 0.7872 & 0.7872 \\
Row factor & 15.0 & \\
& 0.6218 & 9.328 \\
Interaction & 15.0 & \\
& 0.02136 & 0.3204 \\
Residual (error) & 64.0 & \\
Total & 0.001625 & 0.1040 \\
& 95.0 & 10.54
\end{tabular}


Table B.3 Statistical comparison between the distributions of $P$. putida $\mathrm{F} 1$ at $0.2 \mathrm{mM}$ toluene and nonchemotactic $P$. putida $\mathrm{F} 1 \mathrm{CheA}$ in the $\mathrm{CF}-\mu \mathrm{Chip}$, as illustrated in Figure 4.6.

Table Analyzed

Data 1

Two-way ANOVA

Source of Variation

$\%$ of total variation

$P$ value

Interaction

$0.86 \quad 0.0002$

Column factor

$1.85<0.0001$

Row factor

96.29

$<0.0001$

Source of Variation

$\mathrm{P}$ value summary
$* * *$

Significant?

Interaction

Column factor

Row factor

$* * *$

Yes

$* * *$

Yes

Row factor

Source of Variation

Df Sum-of-squares

Mean square

F

Interaction

15

0.08488

0.005659

3.631

Column factor

1

0.1829

0.1829

117.4

Row factor

15

9.533

0.6356

407.8

Residual

64

0.09975

0.001559

Number of missing values

0

Data analyzed: Data 1

Source of Variation

Degrees of Freedom

Sum of Squares

Mean square

Column factor

1.0

0.1829

0.1829

Row factor

15.0

9.533

0.6356

Interaction

15.0

0.08488

Residual (error)

0.005659

64.0

0.09975

Total

0.001559

95.0

9.901 
Table B.4 Statistical comparison between the distributions of E. coli $\mathrm{HCB} 1$ at $3 \mathrm{mM} \alpha-$ methylaspartate and $0.3 \mathrm{mM} \alpha$-methylaspartate in the $\mathrm{CF}-\mu \mathrm{Chip}$, as illustrated in Figure

Table Analyzed

Two-way ANOVA

Source of Variation

Interaction

Column factor

Row factor

Source of Variation

Interaction

Column factor

Row factor

Source of Variation

Interaction

Column factor

Row factor

Residual

Number of missing values

4.8 .

Data analyzed: Data 1

$\underline{\text { Source of Variation }}$

Column factor

Row factor

Interaction

Residual (error)

Total

$\begin{array}{rr}\% \text { of total variation } & \text { P value } \\ 5.69 & <0.0001 \\ 6.19 & <0.0001 \\ 86.45 & <0.0001\end{array}$

$\begin{array}{rr}\mathrm{P} \text { value summary } & \text { Significant? } \\ * * * & \text { Yes } \\ * * * & \text { Yes } \\ * * * & \text { Yes }\end{array}$

Df Sum-of-squares Mean square F

$\begin{array}{llll}16 & 0.5756 & 0.03598 & 14.43\end{array}$

$\begin{array}{llll}1 & 0.6263 & 0.6263 & 251.2\end{array}$

$\begin{array}{llll}16 & 8.749 & 0.5468 & 219.4\end{array}$

$\begin{array}{lll}68 & 0.1695 & 0.002493\end{array}$

0

\begin{tabular}{|c|c|}
\hline Degrees of Freedom & $\underline{\text { Sum of Squares }}$ \\
\hline Mean square & \\
\hline$\overline{1.0}$ & 0.6263 \\
\hline 0.6263 & \\
\hline $\begin{array}{l}16.0 \\
0.5468\end{array}$ & 8.749 \\
\hline $\begin{array}{l}16.0 \\
0.03598\end{array}$ & 0.5756 \\
\hline $\begin{array}{l}68.0 \\
0.002493\end{array}$ & 0.1695 \\
\hline 101.0 & 10.12 \\
\hline
\end{tabular}


Table B.5 Statistical comparison between the distributions of $E$. coli $\mathrm{HCB} 1$ at $3 \mathrm{mM} \alpha-$ methylaspartate and nonchemotactic E. coli $\mathrm{HCB} 437$ in the $\mathrm{CF}-\mu \mathrm{Chip}$, as illustrated in Figure 4.8.

Table Analyzed Data 1

Two-way ANOVA

Source of Variation

Interaction

Column factor

$\begin{array}{rr}\% \text { of total variation } & \text { P value } \\ 3.22 & <0.0001 \\ 7.15 & <0.0001 \\ 88.33 & <0.0001\end{array}$

Source of Variation

$\begin{array}{rr}\text { P value summary } & \text { Significant? } \\ * * * & \text { Yes } \\ * * * & \text { Yes } \\ * * * & \text { Yes }\end{array}$

Column factor

Row factor

Source of Variation

Interaction

Column factor

Row factor

Residual

$\begin{array}{rrrr}\text { Df } & \text { Sum-of-squares } & \text { Mean square } & \text { F } \\ 16 & 0.3659 & 0.02287 & 10.57 \\ 1 & 0.8125 & 0.8125 & 375.6 \\ 16 & 10.03 & 0.6270 & 289.9 \\ 68 & 0.1471 & 0.002163 & \end{array}$

Number of missing values

0

Data analyzed: Data 1

\begin{tabular}{lll} 
Source of Variation & & \multicolumn{2}{c}{ Degrees of Freedom } & Sum of Squares \\
Column factor & $\frac{\text { Mean square }}{1.0}$ & \\
& 0.8125 & 0.8125 \\
Row factor & 16.0 & \\
& 0.6270 & 10.03 \\
Interaction & 16.0 & \\
& 0.02287 & 0.3659 \\
Residual (error) & 68.0 & \\
Total & 0.002163 & 0.1471 \\
& 101.0 & 11.36
\end{tabular}


Table B.6 Statistical comparison between the distributions of E. coli $\mathrm{HCB} 1$ at $0.3 \mathrm{mM} \alpha-$ methylaspartate and nonchemotactic $E$. coli $\mathrm{HCB} 437$ in the $\mathrm{CF}-\mu \mathrm{Chip}$, as illustrated in Figure 4.8.

Table Analyzed

Data 1

Two-way ANOVA

Source of Variation

Interaction

$\%$ of total variation

$P$ value

Column factor

$9.62<0.0001$

$25.08<0.0001$

Row factor

64.34

$<0.0001$

Source of Variation

Interaction

Column factor

$\begin{array}{rr}\text { P value summary } & \text { Significant? } \\ * * * & \text { Yes } \\ * * * & \text { Yes } \\ * * * & \text { Yes }\end{array}$

Row factor

Source of Variation

Interaction

Column factor

Row factor

Residual

Number of missing values

Data analyzed: Data 1

$\underline{\text { Source of Variation }}$

Column factor

Row factor

Interaction

Residual (error)

Total
0

Degrees of Freedom Sum of Squares

Mean square

1.0

2.865

16.0

0.4594

16.0
Df Sum-of-squares Mean square $\mathrm{F}$

16

1.099

0.06867

42.40

1

2.865

2.865

1769

16

7.351

0.4594

283.7

$0.1101 \quad 0.001620$

$\mathrm{F}$

68
0.06867

68.0

0.001620

101.0
2.865

7.351

1.099

0.1101

11.42 


\section{APPENDIX C: TWO-WAY ANOVA ANALYSIS IN H- $\mu$ CHIP}

Table C.1 Statistical comparison between the distributions of $P$. putida $\mathrm{F} 1$ and nonchemotactic $P$. putida $\mathrm{F} 1 \mathrm{CheA}$ at the junctions in the $\mathrm{H}-\mu \mathrm{Chip}$ at $0.5 \mathrm{~m} / \mathrm{d}$, as illustrated in Figure 5.6.

Table Analyzed

Data 1

Two-way ANOVA

Source of Variation

Interaction

$\%$ of total variation

$P$ value

2.96

0.9196

Column factor

66.33

$<0.0001$

Row factor

3.22

0.9019

Source of Variation

$P$ value summary

Significant?

Interaction

Column factor

$\begin{array}{rr}\text { ns } & \text { No } \\ * * * & \text { Yes } \\ \text { ns } & \text { No }\end{array}$

Row factor

DfSum-of-squares Mean square

Source of Variation

$7 \quad 0.003568$

0.0005097

0.3538

Column factor

$\begin{array}{lrr}1 & 0.07996 & 0.07996\end{array}$

55.50

Row factor

7

Residual

23

0.003878

0.0005539

0.3845

Number of missing values

6

Data analyzed: Data 1

\begin{tabular}{lll} 
Source of Variation & & \multicolumn{2}{l}{ Degrees of Freedom } & Sum of Squares \\
Column factor & $\frac{\text { Mean square }}{1.0}$ & \\
& 0.07996 & 0.07996 \\
Row factor & 7.0 & \\
& 0.0005539 & 0.003878 \\
Interaction & 7.0 & \\
& 0.0005097 & 0.003568 \\
Residual (error) & 23.0 & 0.03314 \\
Total & 0.001441 & \\
& 38.0 &
\end{tabular}


Table C.2 Statistical comparison between the distributions of $P$. putida $\mathrm{F} 1$ at the junctions in the H- $\mu$ Chip at both $0.5 \mathrm{~m} / \mathrm{d}$ and $5 \mathrm{~m} / \mathrm{d}$, as illustrated in Figure 5.7 .

Table Analyzed

Data 1

Two-way ANOVA

Source of Variation

$\%$ of total variation

$P$ value

Interaction

$\begin{array}{ll}7.84 & 0.1918\end{array}$

Column factor

$63.55<0.0001$

Row factor

5.25

0.4318

Source of Variation

$P$ value summary

Significant?

Interaction

Column factor

Row factor

$\begin{array}{rr}\text { ns } & \text { No } \\ * * * & \text { Yes } \\ \text { ns } & \text { No }\end{array}$

Source of Variation

DfSum-of-squares Mean square

Interaction

Column factor

$\begin{array}{lll}7 & 0.006007 & 0.0008582\end{array}$

Row factor

$0.04872 \quad 0.04872$

87.02

Residual

$\begin{array}{lll}7 & 0.004025 & 0.0005749\end{array}$

1.027

Number of missing values

$\begin{array}{lll}32 & 0.01791 & 0.0005598\end{array}$

0

Data analyzed: Data 1

Source of Variation

Column factor

Row factor

Interaction

Residual (error)

Total

\begin{tabular}{lll}
\cline { 1 - 1 } Degrees of Freedom square & & Sum of Squares \\
\hline 1.0 & & 0.04872 \\
0.04872 & & \\
7.0 & 0.004025 \\
0.0005749 & \\
7.0 & 0.006007 \\
0.0008582 & \\
32.0 & 0.01791 \\
0.0005598 & \\
47.0 & 0.07666
\end{tabular}


Table C.3 Statistical comparison between the breakthrough curves of $P$. putida $\mathrm{F} 1$ and nonchemotactic CheA at the outlet in the $\mathrm{H}-\mu \mathrm{Chip}$ at $0.25 \mathrm{~m} / \mathrm{d}$, as illustrated in Figure 5.10 .

Table Analyzed

Data 1

Two-way ANOVA

Source of Variation

Interaction

$\begin{array}{rr}\% \text { of total variation } & \text { P value } \\ 0.71 & 0.3853 \\ 3.61 & <0.0001 \\ 92.82 & <0.0001\end{array}$

Row factor

$\begin{array}{rr}\text { P value summary } & \text { Significant? } \\ \text { ns } & \text { No } \\ * * * & \text { Yes } \\ * * * & \text { Yes }\end{array}$

Source of Variation

DfSum-of-squares Mean square

Interaction

Column factor

$0.04684 \quad 0.005205$

Row factor

1

$0.2390 \quad 0.2390$

50.48

Residual

9

$\begin{array}{ll}6.146 & 0.6828\end{array}$

144.2

Number of missing values

40

$0.1894 \quad 0.004735$

0

Data analyzed: Data 1

$\underline{\text { Source of Variation }}$

Column factor

Row factor

Interaction

Residual (error)

Total

\begin{tabular}{|c|c|}
\hline \multirow{2}{*}{\multicolumn{2}{|c|}{$\frac{\text { Degrees of Freedom }}{\text { Mean square }}$}} \\
\hline & \\
\hline $\begin{array}{l}1.0 \\
0.2390\end{array}$ & 0.2390 \\
\hline $\begin{array}{l}9.0 \\
0.6828\end{array}$ & 6.146 \\
\hline $\begin{array}{l}9.0 \\
0.005205\end{array}$ & 0.04684 \\
\hline $\begin{array}{l}40.0 \\
0.004735 \\
590\end{array}$ & 0.1894 \\
\hline 59.0 & 6.621 \\
\hline
\end{tabular}


Table C.4 Statistical comparison between the breakthrough curves of $P$. putida $\mathrm{F} 1$ and nonchemotactic CheA at the outlet in the H- $\mu$ Chip at $5 \mathrm{~m} / \mathrm{d}$, as illustrated in Figure 5.10.

Table Analyzed

Data 1

Two-way ANOVA

Source of Variation

Interaction

$\%$ of total variation $\quad \mathrm{P}$ value

Column factor

0.33

0.7194

Row factor

0.02

0.5080

$97.49<0.0001$

Source of Variation

$\mathrm{P}$ value summary

Significant?

Interaction

$\begin{array}{rr}\mathrm{ns} & \text { No } \\ \mathrm{ns} & \text { No } \\ * * * & \text { Yes }\end{array}$

Row factor

Yes

Source of Variation

Df Sum-of-squares Mean square

Interaction

Column factor

Row factor

Residual

$\begin{array}{llll}1 & 0.001271 & 0.001271 & 0.4462\end{array}$

$9 \quad 5.160 \quad 0.5733 \quad 201.2$

$40 \quad 0.1140 \quad 0.002849$

Number of missing values

0

Data analyzed: Data 1

\begin{tabular}{|c|c|c|}
\hline \multirow[t]{2}{*}{ Source of Variation } & Degrees of Freedom & $\underline{\text { Sum of Squares }}$ \\
\hline & Mean square & \\
\hline \multirow[t]{2}{*}{ Column factor } & 1.0 & 0.001271 \\
\hline & 0.001271 & \\
\hline \multirow[t]{2}{*}{ Row factor } & 9.0 & 5.160 \\
\hline & 0.5733 & \\
\hline \multirow[t]{2}{*}{ Interaction } & 9.0 & 0.01752 \\
\hline & 0.001947 & \\
\hline \multirow[t]{2}{*}{ Residual (error) } & 40.0 & 0.1140 \\
\hline & 0.002849 & \\
\hline Total & 59.0 & 5.292 \\
\hline
\end{tabular}


Table C.5 Statistical comparison between the breakthrough curves of $P$. putida F1 at the outlet in the H- $\mu$ Chip at both 0.25 and $5 \mathrm{~m} / \mathrm{d}$, as illustrated in Figure 5.10 .

Table Analyzed

Data 1

Two-way ANOVA

Source of Variation

$$
\begin{array}{rr}
\% \text { of total variation } & \mathrm{P} \text { value } \\
6.22 & <0.0001 \\
3.95 & <0.0001 \\
87.57 & <0.0001
\end{array}
$$

Interaction

Column factor

Row factor

Source of Variation

Interaction

Column factor

Row factor

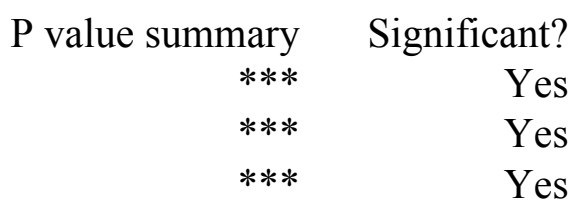

Source of Variation

Interaction

Column factor

DfSum-of-squares Mean square

Row factor

12.22

Residual

9

$0.3818 \quad 0.04243$

69.90

Residual

40

$\begin{array}{ll}5.378 & 0.5976\end{array}$

172.1

\begin{tabular}{|c|c|c|}
\hline Source of Variation & $\frac{\text { Degrees of Freedom }}{\text { Mean square }}$ & $\underline{\text { Sum of Squares }}$ \\
\hline Column factor & $\begin{array}{l}1.0 \\
0.2427\end{array}$ & 0.2427 \\
\hline Row factor & $\begin{array}{l}9.0 \\
0.5976\end{array}$ & 5.378 \\
\hline Interaction & $\begin{array}{l}9.0 \\
0.04243\end{array}$ & 0.3818 \\
\hline Residual (error) & $\begin{array}{l}40.0 \\
0.003472\end{array}$ & 0.1389 \\
\hline Total & 59.0 & 6.142 \\
\hline
\end{tabular}

Number of missing values

0

Data analyzed: Data 1 


\section{APPENDIX D: CORRELATION BETWEEN OPTICAL DENSITY}

\section{AND LIGHT INTENSITY IN THE $\mu$ CHIP}

One conventional way to determine bacterial concentration is to count individual cells, which is time-consuming and error-prone. Instead, for this analysis, it was assumed that the gray level intensity of each cell was similar to each other in the population, thus allowing the light intensity to be correlated with the bacterial concentration in terms of optical density.

The background light intensity decreased the contrast of the bacteria, so the filters provided with Image $\mathrm{J}$ software were used to remove the background. Image of bacterial distribution without the background is presented as Figure D.1 (a). The white dots in the image are the bacterial cell bodies. Because the bacteria were discrete points, 150 images were taken at the same experimental condition, and then averaged using Image $J$ to yield a single image as shown in Figure D.1 (b). This process reduced the statistical variation in the calibration between bacterial concentration and light intensity.
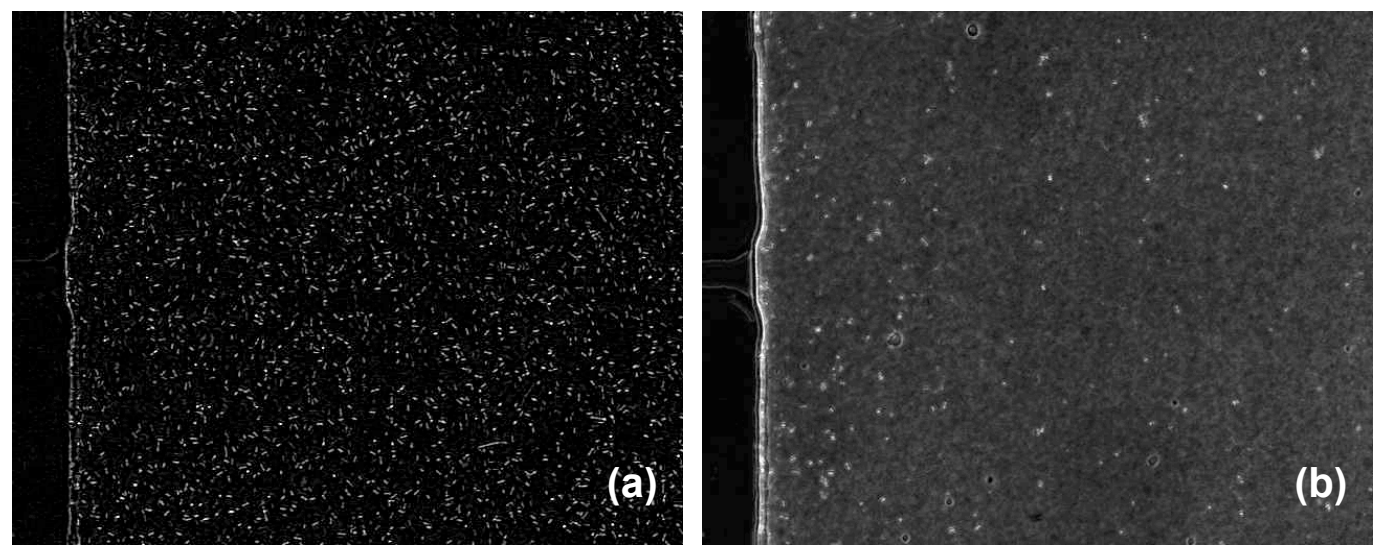

Figure D.1 (a) Background removed by Image J. The white spots are the bacteria. (b) The image averaged from 150 images similar to (a) under the same experimental condition. 
Five different concentrations of the bacterial suspension were prepared. The optical density of the bacterial suspension was measured directly by a spectrometer, and the bacterial light intensity inside the microfluidic device was obtained from the ROI in Figure D.2, when there was no attractant. The correlation between the optical density and the light intensity is shown in Figure D.3.

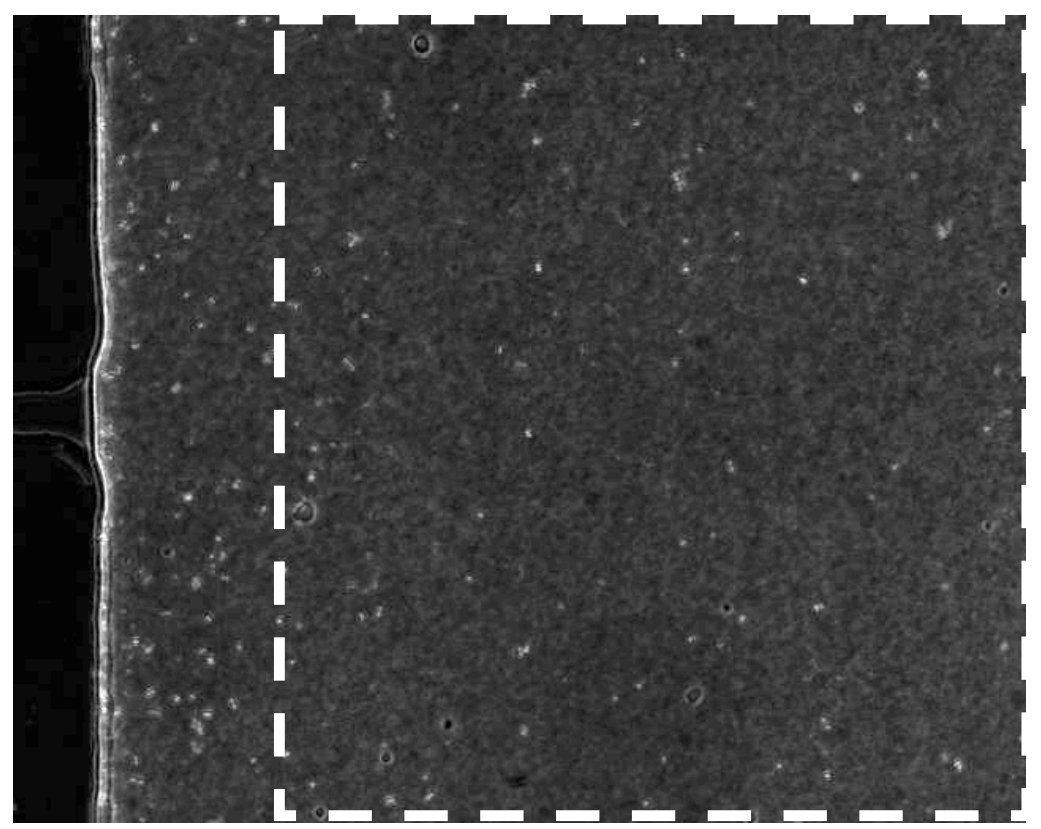

Figure D.2 The ROI is the square formed by the dashed line, and the integrated intensity in the ROI was obtained by Image $\mathrm{J}$ for five different bacterial concentrations. 


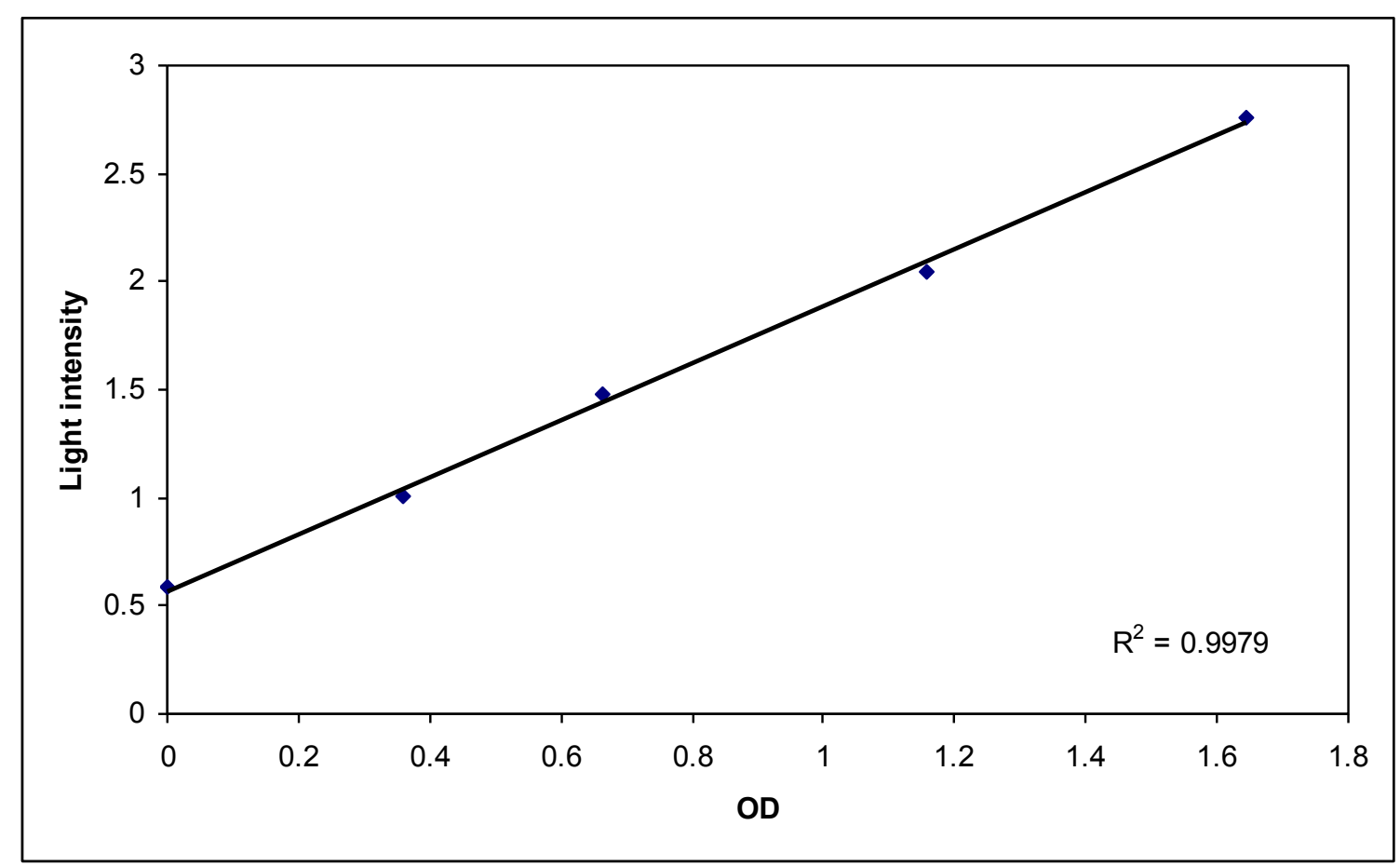

Figure D.3 Correlation between the optical density (OD) and the light intensity of bacterial concentration in the microfluidic device.

The bacterial concentration and the light intensity from the image had a strongly positive correlation. Therefore, the light intensity could be used to represent the bacterial concentration. However, in Figure D. 2 the value of light intensity at $\mathrm{OD}=0$ was larger than zero, which meant that there was still some background noise from the image after the first background removal; thus, a second background removal was implemented in the following data analysis. 


\section{APPENDIX E: VALIDATION OF BACTERIA/ATTRACTANT SYSTEM, IMAGE ANALYSIS AND EXPERIMENTAL SETUP}

\section{E.1 Introduction}

The main purpose of this chapter is to document the results from the preliminary tests for the bacteria/attractant pairs used in this study, the methodology for data analysis and the flow study in the microfluidic devices.

Section E.2 reviews the quality of the various fluorescence staining used for bacteria. Section E.3 presents the correlation between fluorescence intensity and bacterial density and between optical density and bacterial density, which validates the methodology for image analysis. Section E.4 summarizes the impact of the membrane dye FM 4-64 on bacterial motility and suggests the correct way to use the molecular probe in this study. Section E.5 introduces the collaborative work on the generic transformation of GFP into P. putida strains and related problems. Section E.6 provides the simulation results about the flow patterns in microfluidic devices ( $\mu$ Chip).

\section{E.2 Bacterial Fluorescence}

The quality of bacterial fluorescence was checked using the microscopic setup mentioned in Section 3.6. The images from both fluorescent view and bright field view are shown in Figure E.1. All fluorescence labeled cells produced a strong signal that was easily distinguished from the background. The calibration between the bacterial fluorescence and bacterial density is given in Figure E.2 in section E.2. 

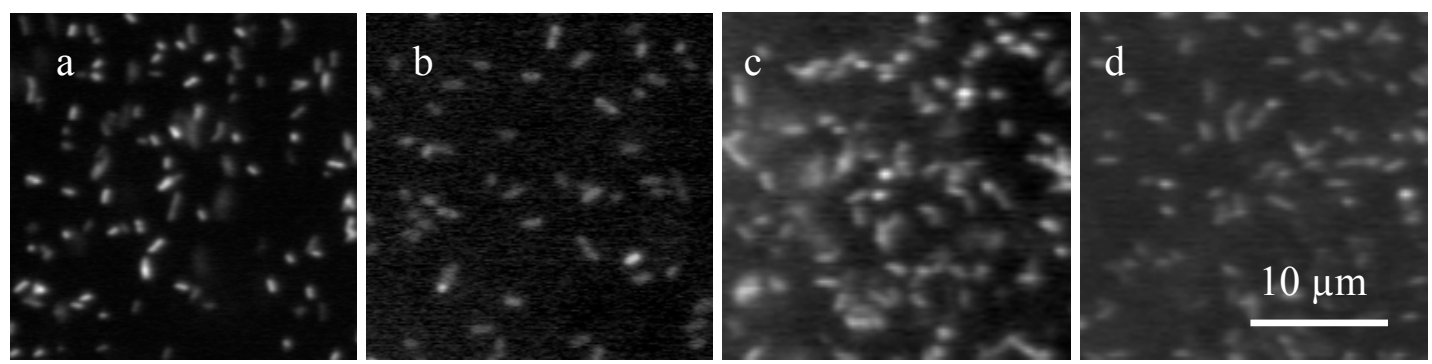

Figure E.1 (a) P. putida F1 stained with FM 4-64, (b) P. putida F1 CheA stained with DAPI, (c) E. coli HCB1 tagged with GFP and (d) E. coli HCB437 stained with DAPI under $20 \times$ objective.

\section{E.3 Optical Density vs. Bacterial Density vs. Fluorescence}

As mentioned in Chapter 3, the correlation was determined by using GFP tagged $E$. coli bacteria. The correlation between the $\mathrm{OD}_{590}$ and bacterial density is shown in Figure E.2a with a $\mathrm{R}^{2}$ equaling to 0.99 , and the correlation between normalized GFP intensity and bacterial density (Figure E.2b) also have a very high correlation coefficient $\left(\mathrm{R}^{2}=\right.$ 0.99), and these results mean that both OD value and GFP fluorescence can represent the actual bacterial density in the working range. With the confidence from the results about GFP fluorescence, it is reasonable to deduce that the FM 4-64 and DAPI stains should also be representative of bacterial density. 


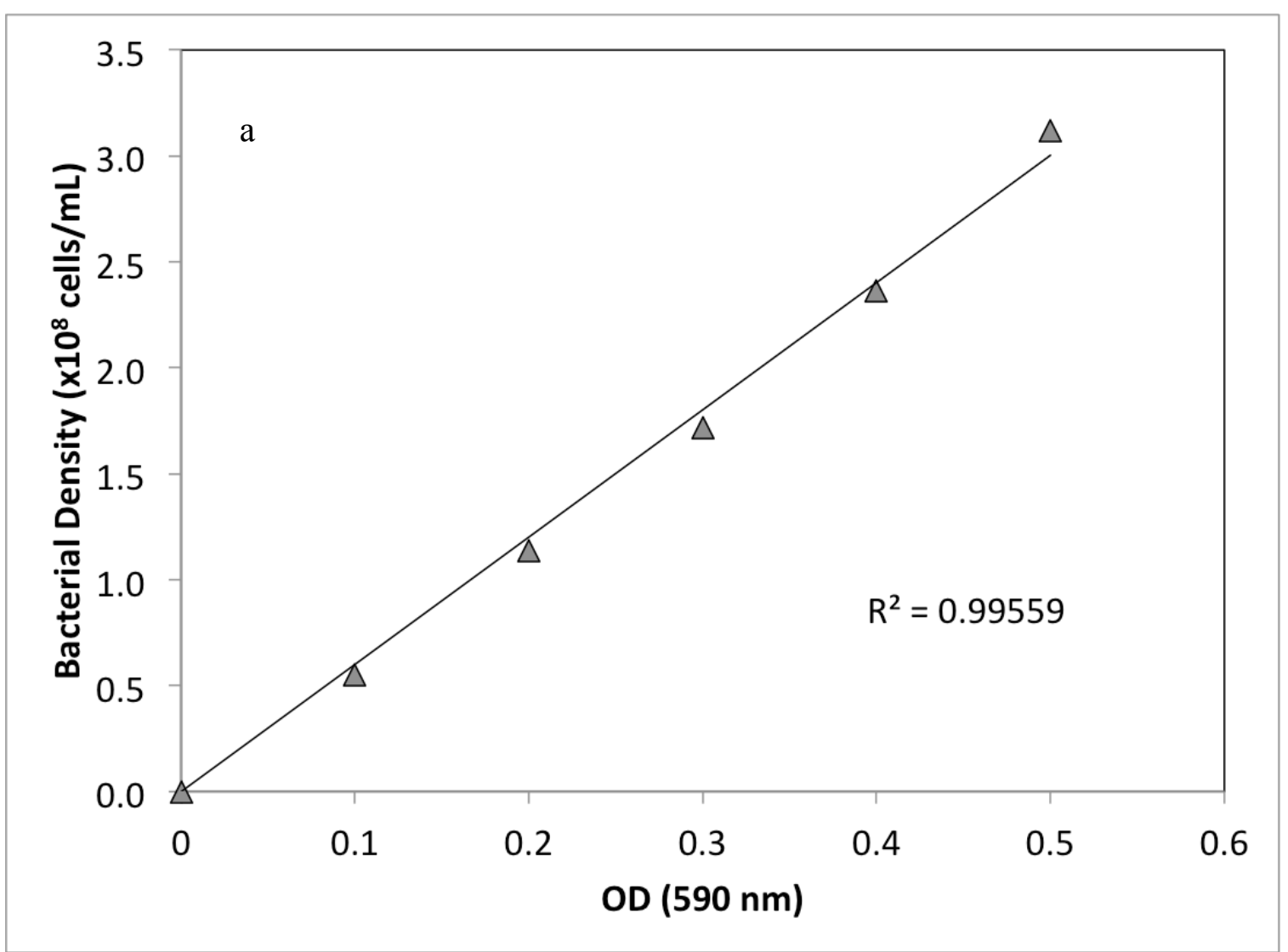




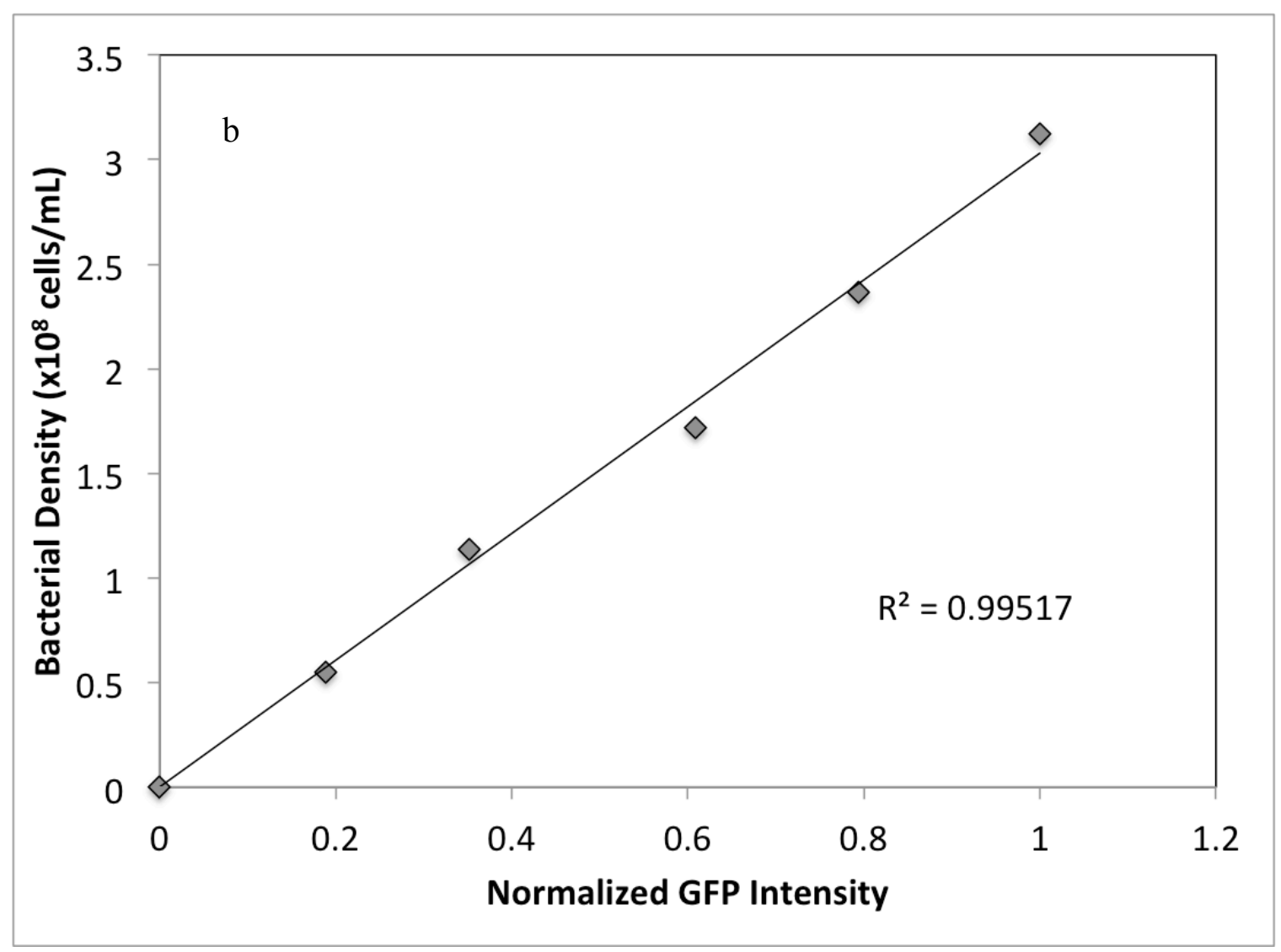

Figure E.2 Correlations between (a) $\mathrm{OD}_{590}$ and bacterial density and between (b) normalized GFP intensity and bacterial density. The triangle and diamond points are the experimental data, and the straight lines are the corresponding fitting curves.

\section{E.4 FM 4-64 Impact on Bacterial Motility}

The dye FM 4-64 itself did not affect the motility. Stained and unstained E. coli HCB1 with GFP from same batch were mixed and injected into one inlet of the CF$\mu$ Chip, and $10 \%$ RMB buffer was injected into the other inlet. Images of bacterial distribution in the cross channel was taken for both GFP and FM 4-64 fluorescence at 0 and 25 minutes of injection, and images at time 0 was chosen as the background noise and subtracted from images at time 25 minutes, and then the results of the processed 
images were plotted in Fig. E.3. The two groups of bacteria followed the same trend and could not differentiate from each other based on the statistical analysis (two-way ANOVA), and the experimental data also matched the simulation result using reported value of bacterial random motility. Both the stained and unstained bacteria had the similar motility and the dye itself did not affect the bacterial motility. The unstained bacteria had a lower population density than the stained ones from 1 to $1.5 \mathrm{~mm}$, and that was induced by experimental errors. Some GFP fluorescence might inadvertently be subtracted when removing the background noise, because the signal-to-noise ratio of GFP was lower than FM 4-64. This difference from 1 to $1.5 \mathrm{~mm}$ was also not significant according to statistical analysis.

The experimental data were then fitted using Eqn 4.4, and the best fitting were determined using the method of least squares, and the values of bacterial random motility were reported accordingly as following. Then the uncertainty of bacterial random motility was determined by allowing a $10 \%$ increase in the value of the least squares, then the fitting gave us the random motility $=1.1 \pm 0.2 \times 10^{-10} \mathrm{~m}^{2} / \mathrm{s}$ for the FM 4-64 stained bacteria and $0.7 \pm 0.2 \times 10^{-10} \mathrm{~m}^{2} / \mathrm{s}$ for the unstained bacteria. The student $t$-test showed that there was no significant difference between the two values, so that meant that FM 464 had no impact on bacterial motility. 


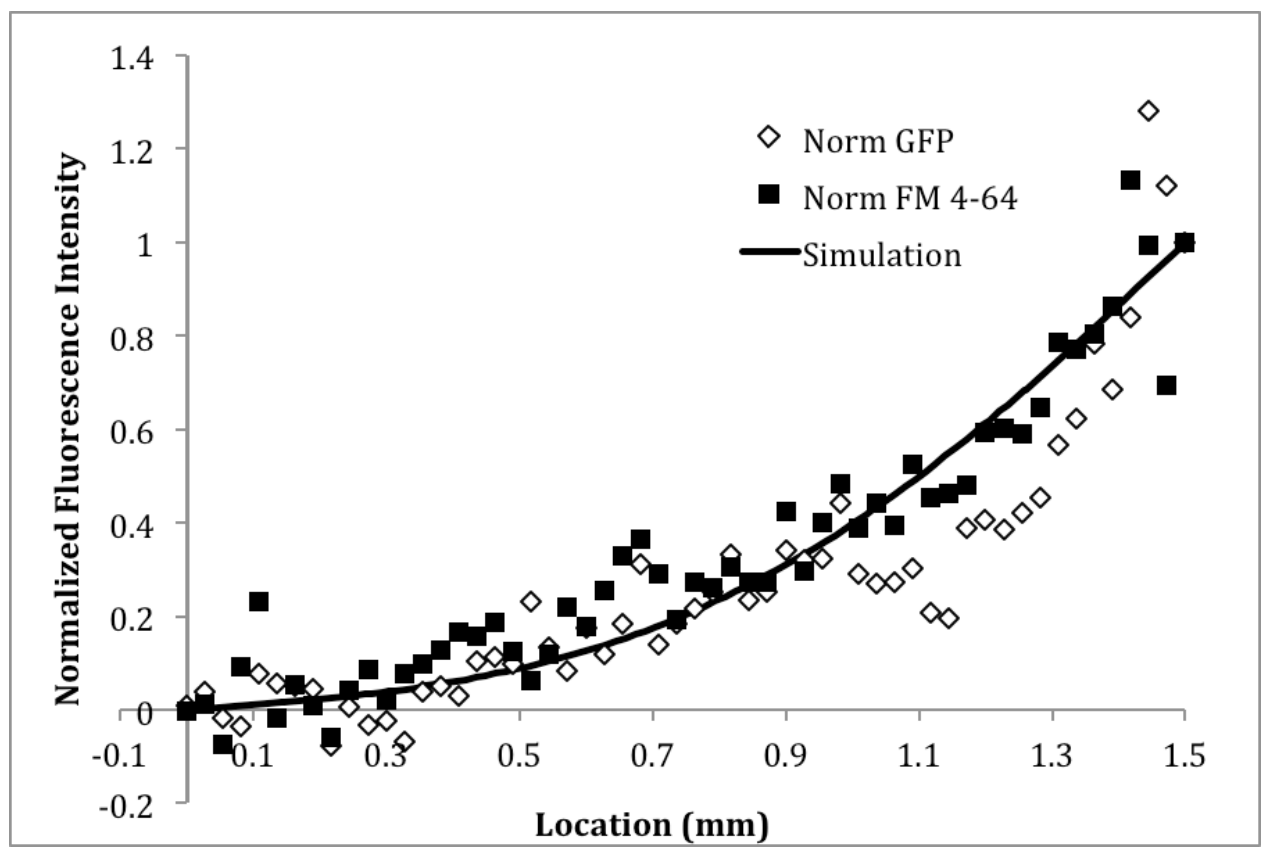

Figure E.3 Normalized fluorescence intensities from GFP (blank diamond) and FM 4-64 (black square) in the cross channel at 25 minutes of injection. The solid curve represents the fitting result for the FM 4-64 stained bacteria, and the dash line for the unstained bacteria (only tagged with GFP).

An interesting result about FM 4-64 staining was that although the membrane dye itself did not hurt the bacterial properties, the excitation of fluorescence might damage the bacterial motility. The reason was probably because the FM 4-64 dye was linked to the plasmatic membrane, and the fluorescence excitation generated extremely high level of energy absorbance and energy release in a very short time, which could easily lead to malfunctions of the cell membrane. Although no evidence indicated that the high-energy release might kill the cell, the bacterial flagella could be greatly impacted as they located peritrichously on the cell membrane. This suggested that the bacterial fluorescence from FM 4-64 could not be checked in real time in CF- $\mu$ Chip, as the bacterial distribution was 
purely established by their motility, and there was also no renewal in the bacterial population; the fluorescence should be checked at the end of the experiments for this microfluidic device. For $\mathrm{H}-\mu \mathrm{Chip}$, the fluorescent intensity still could be recorded in real time at the outlet of microfluidic device, so that the excitation would not affect the functioning bacteria in the main channels. The dimension of the affected zone was determined by the arc lamp precision and specific objective, and it could be directly measured from the bright area covered by the focused light beam. As shown in Figure E.4, the affected zone (the green circle) has a diameter of about 2-3 $\mathrm{mm}$, and it is far away from the main channel and porous network. Although there should be scattered arc lamp beam all over the device, but its intensity is much weaker than the focused affected zone. Bacterial movement in the main channel and porous network was actually examined in bright field, and the motility was not affected at all.

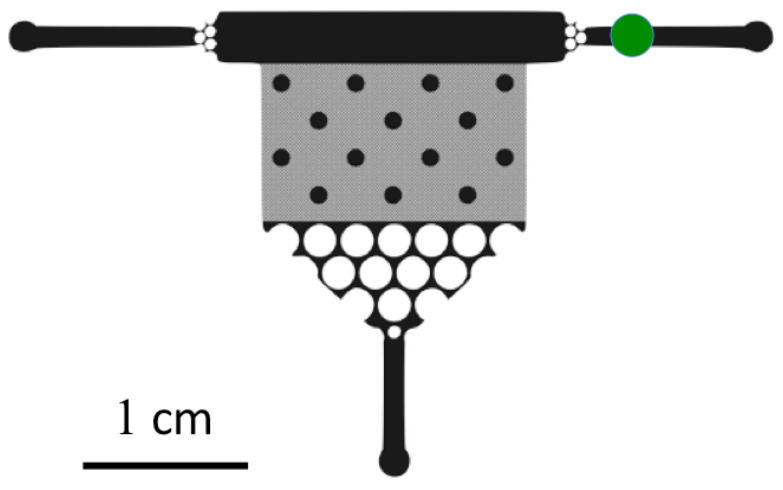

Figure E.4 Affected zone (the green circle) by arc lamp light beam on the H- $\mu$ Chip.

\section{E.5 GFP Transformation to $P$. putida}

Besides the E. coli strains, GFP transformation was tried in P. putida strains too. The protocol was the same as stated in the Bio-Rad pGLO bacterial transformation kit. 
Generally, a pGLO plasmid containg the genes for expressing both GFP and ampicillin resistence were shot into the cell body by heat shcok. However, the plasmid transformation was not successful due to the biological stress inside the $P$. putida bacterium. Dr. Joshua Shrout tried to tag these strains chromosomally with a $\operatorname{Tn} 7$ transposon insert at the site adjacent to the gene glmS on the Pseudomonas chromosome. The details of this transformation were well documented by Schweizer's group at Colorado State (Hoang et al., 1998). Genes of antibiotic selection was also tagged into the bacterial chromosome, but they were not required to maintain fluorescence and/or prevent loss; they were required for conjugation. In this case, both $P$. putida $\mathrm{F} 1$ and CheA mutant were tagged with GFP and kanamycin-streptomycin resistant; another CheA mutant sample was tagged with GFP and gentamycin resistant.

Both the chemotactic and nonchemotactic $P$. putida strains successfully expressed GFP after the inoculation in the modified Hutner's Mineral Base, which was mentioned in Chapter 3. However, the GFP expression was not strong compared to that in E. coli strains, and only about one third of the overall bacterial population showed the fluorescence, even with the selective help of corresponding antibiotic addition. More critically, the GFP labeled P. putida strains became less motile, or almost non-motile under both the bright field and fluorescence observation. Thus, more work needed to be done to improve the fluorescence quality, as well as to maintain the motility of GFP labeled $P$. putida, and it would be worthwhile to use them in many different circumstances in future research. 


\section{E.6 Simulated Flow Patterns in the $\mu$ Chips}

Both the convection free gradient generator ( $\mathrm{CF}-\mu \mathrm{Chip})$ and the heterogeneous microfluidics (H- $\mu$ Chip) involved with injection flows to achieve their functions. $\mathrm{H}$ $\mu$ Chip studied the impact of flow rates on bacterial distribution in the microfluidic device; the main function of $\mathrm{CF}-\mu \mathrm{Chip}$ was to study bacterial chemotaxis in a no-flow condition, but the no-flow environment was achieved by the two balanced flow streams in the upper channel. Thus, it was important to understand the flow patterns in the microfluidic devices at the first step. The flow patterns were hard to illustrate directly by experimental methods, so a finite-element solver COMSOL 4.3 was used to solve the two dimensional Navier-Stokes equation and the Advection and Diffusion equations.

\section{E.6.1 CF- $\mu$ Chip}

As mentioned in Chapter 3 and illustrated by Figure 3.4, bacteria suspension and chemical solution were injected from two parallel and symmetric inlets, and the injection velocity was comparable to $0.38 \mathrm{~mm} / \mathrm{s}$ in the top channel. Figure E.5 shows how the velocity field distributes in the top channel, as the velocity at the centerline is about 1.5 times larger than the average velocity of $0.38 \mathrm{~mm} / \mathrm{s}$, and the velocity reduces to zero at the sidewalls due to the no slip condition. More importantly, the velocity field can be used to examine the mass transfer between the streams of bacteria and chemical. 
Surface: Velocity magnitude (m/s) Arrow Surface: Velocity field

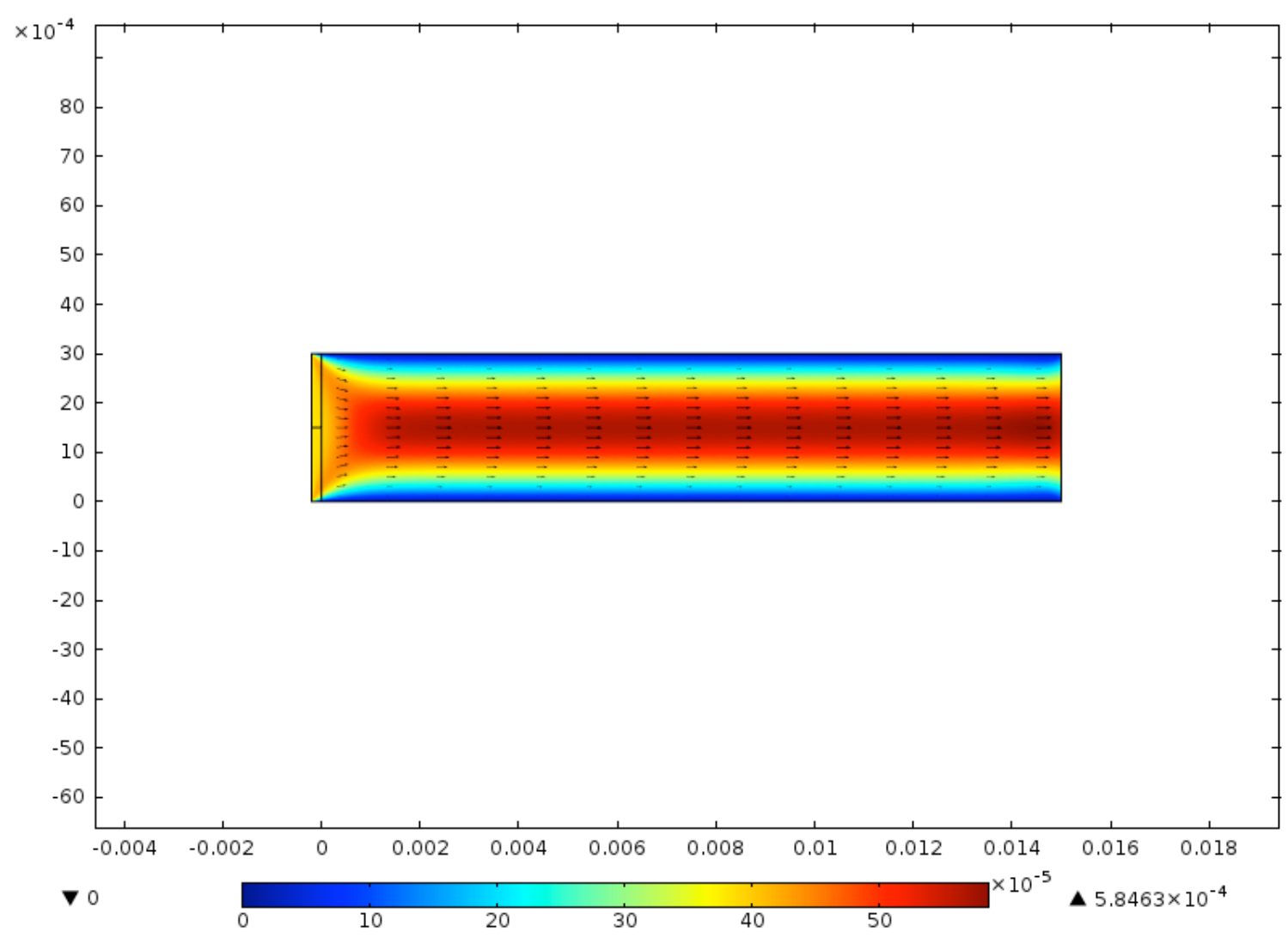

Figure E.5 Simulated velocity field in the top channel of the CF- $\mu$ Chip. Colors toward the red end of the legend bar mean higher values, and colors toward the blue end mean lower values. The black arrows represent the approximate vectors of velocity at certain locations.

P. putida and toluene system was tested first for their distribution under the flow rate (no chemotaxis), and the simulated results were indicated by Figure E.6 and E.7. The bacterial random motility is $1.6 \times 10^{-9} \mathrm{~m}^{2} / \mathrm{s}$ for $P$. putida (Wang and Ford, 2009) and attractant diffusion coefficient is $9.5 \times 10^{-10} \mathrm{~m}^{2} / \mathrm{s}$ for toluene (Montgomery, 1996). Toluene was injected from the upper left inlet, and P. putida from the lower left inlet, and they gradually diffused into each other as the flow moved along the channel. 
Surface: Concentration $\left(\mathrm{mol} / \mathrm{m}^{3}\right)$

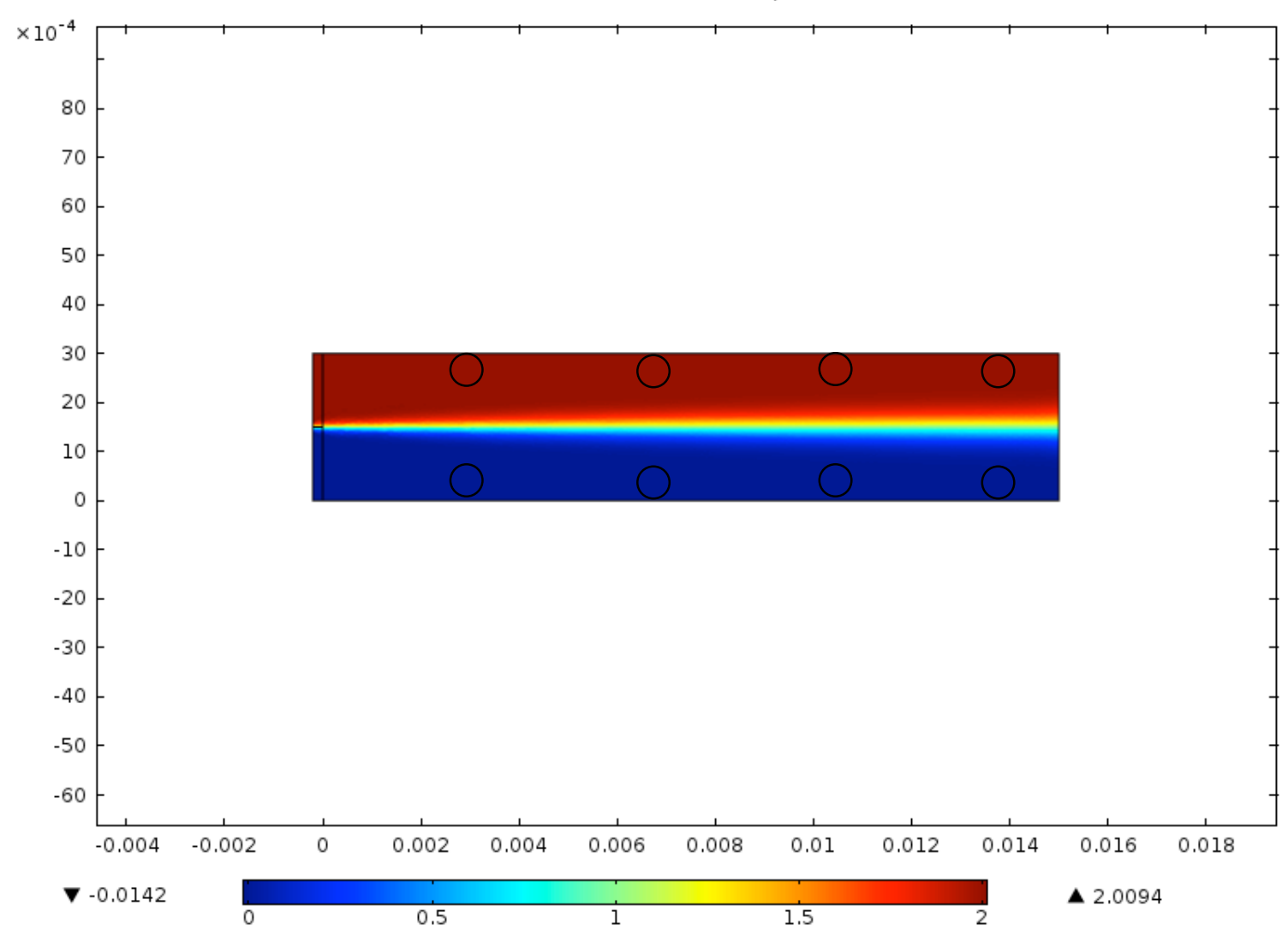

Figure E.6 Simulated distribution of toluene solution in the top channel of the CF- $\mu$ Chip.

Colors toward the red end of the legend bar mean higher values, and colors toward the blue end mean lower values. The rainbow color in the middle of the channel means the diffusion zone. The eight black circles indicate the sizes and locations of the vias for the cross channels in the bottom layer. 
Surface: Concentration $\left(\mathrm{mol} / \mathrm{m}^{3}\right)$

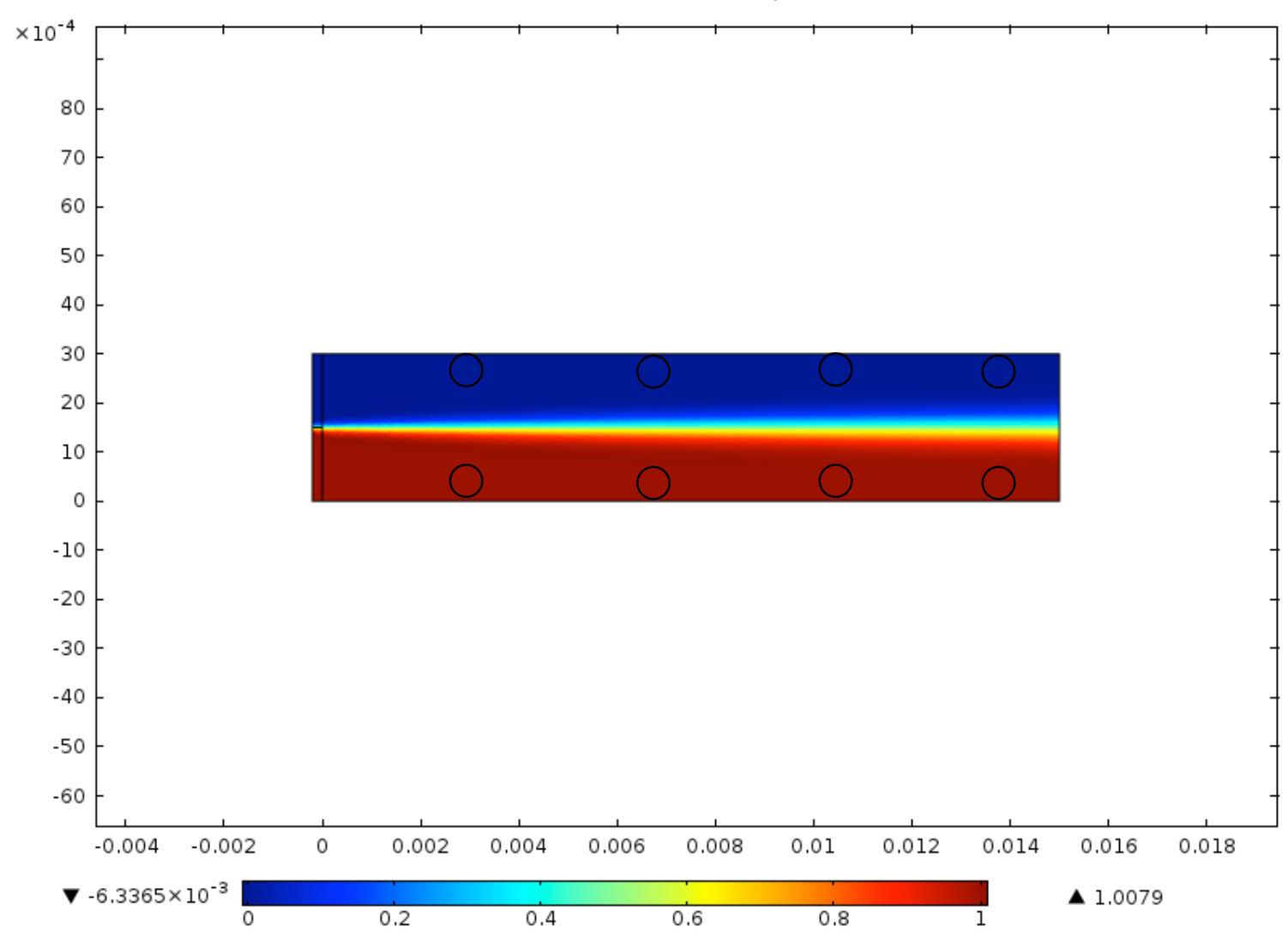

Figure E.7 Simulated distribution of $P$. putida suspension in the top channel of the CF$\mu$ Chip. Colors toward the red end of the legend bar mean higher values, and colors toward the blue end mean lower values. The rainbow color in the middle of the channel means the diffusion zone. The eight black circles indicate the sizes and locations of the vias for the cross channels in the bottom layer.

The diffusing zones indicated from the figures above were expected not to interfere with the vias locating besides the sidewalls. The simulated data of transverse distribution for bacteria and chemical were collected at the outlet on the right side of the channel, and the distribution curves were plotted in Figure E.8. The x-axis stands for the different location points along the transverse direction of the $3 \mathrm{~mm}$ wide channel. 

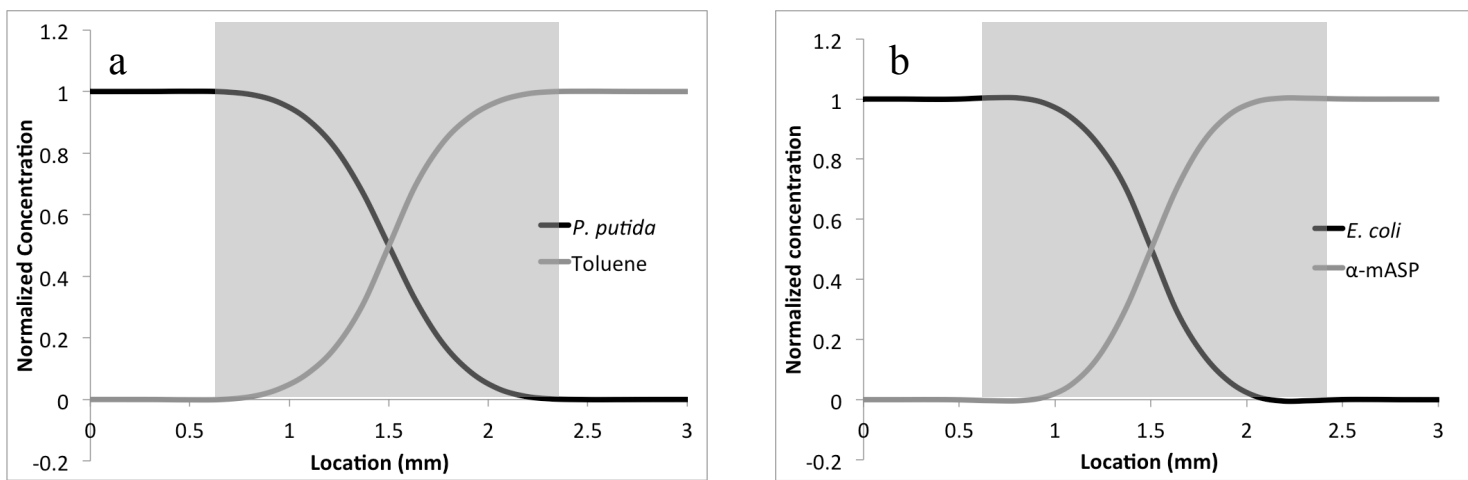

Figure E.8 Simulated distribution of both bacteria and chemical on the transverse direction at the outlet of the top channel. (a) is for P. putida with toluene and (b) is for $E$. coli with $\alpha$-methylaspartate ( $\alpha$-mASP).

E. coli and $\alpha$-mASP system was also checked the same simulation procedure, and the results were similar to $P$. putida and toluene, except their diffusion zone was a bit narrower than the other pair. The bacterial random motility is $3.0 \times 10^{-10} \mathrm{~m}^{2} / \mathrm{s}$ for $E$. coli HCB1 and attractant diffusion coefficient is $8.6 \times 10^{-10} \mathrm{~m}^{2} / \mathrm{s}$ for $\alpha-\mathrm{mASP}$ (Long and Ford, 2009). In either situation, the diffusion zone does not interfere with the vias, because the diameter of via is $600 \mu \mathrm{m}$, so the vias are within the unshaded region (Figure E.8) and should be able to maintain pure solution/suspension concentration.

\section{E.6.2 H- $\mu$ Chip}

The flow pattern is simulated in the heterogeneous microfluidic structure at different flow rates, as shown in Figure E.9 and E.10. The blue square area stands for the porous matrix with proper values of permeability and porosity, which are within the typical range for contaminant layers composed of sand and gravel. The values of permeability and porosity are listed in Table 6.1. The flow in the porous matrix is fairly 
small compared to the major flow in the horizontal channel under either 0.25 or $5 \mathrm{~m} / \mathrm{d}$.

These simulation results on flow patterns revealed an expected flow environment, where the major bulk fluid flowed in the highly permeable horizontal channel, and only a small portion of flow distributed in the porous region with less permeability. However, the study would show that the small portion of flow could also deliver bacteria to the whole porous matrix and it also helped chemotactic bacteria stay longer in this region in presence of NAPL attractant ganglia.

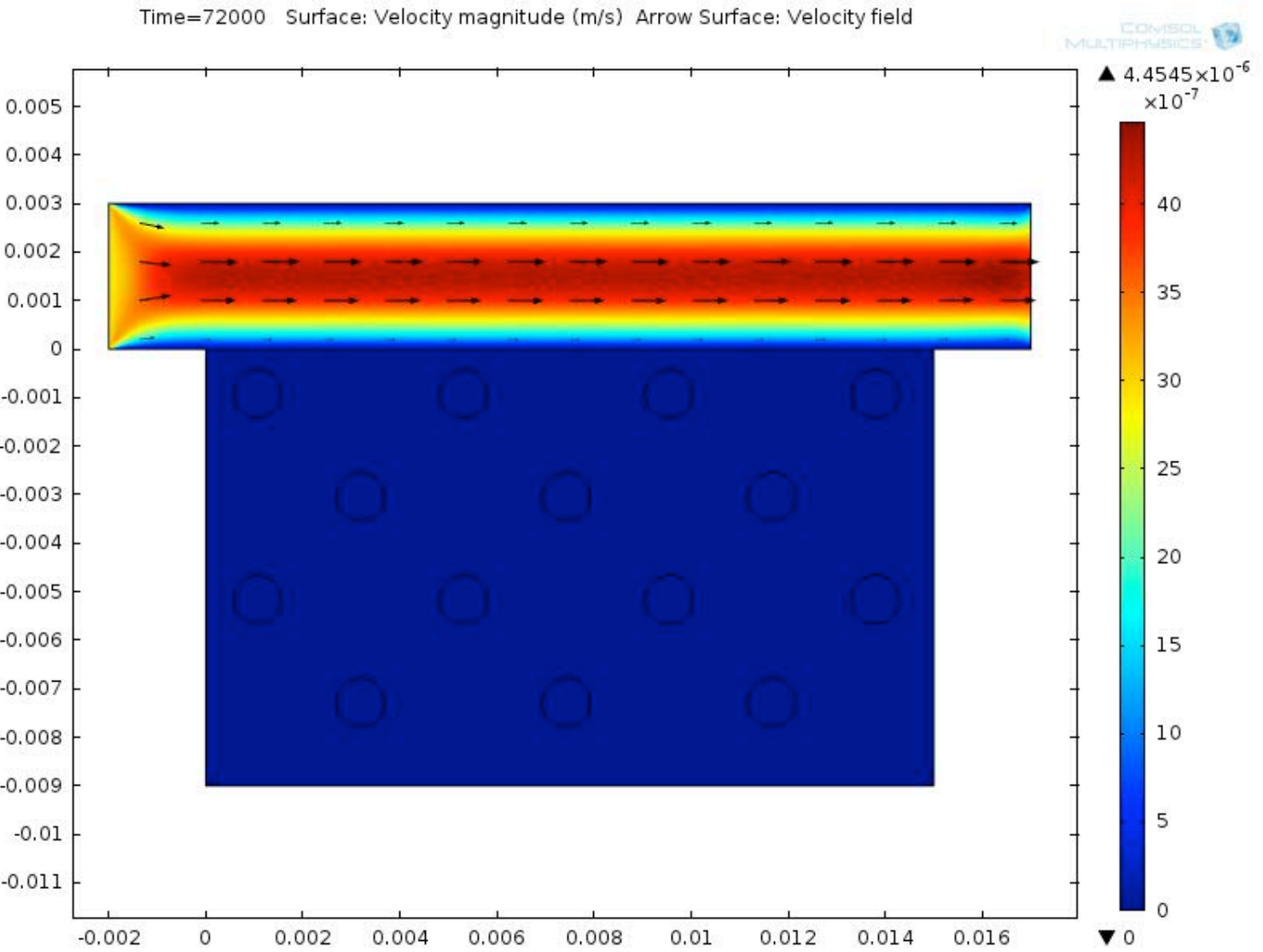

Figure E.9 The simulated flow pattern at $0.25 \mathrm{~m} / \mathrm{d}$ in the $\mathrm{H}-\mu \mathrm{Chip}$. Colors toward the red end of the legend bar mean higher values, and colors toward the blue end mean lower values. The black arrows represent the approximate vectors of velocity at certain locations. 
Time $=72000$ Surface: Velocity magnitude $(\mathrm{m} / \mathrm{s})$ Arrow Surface: Velocity field

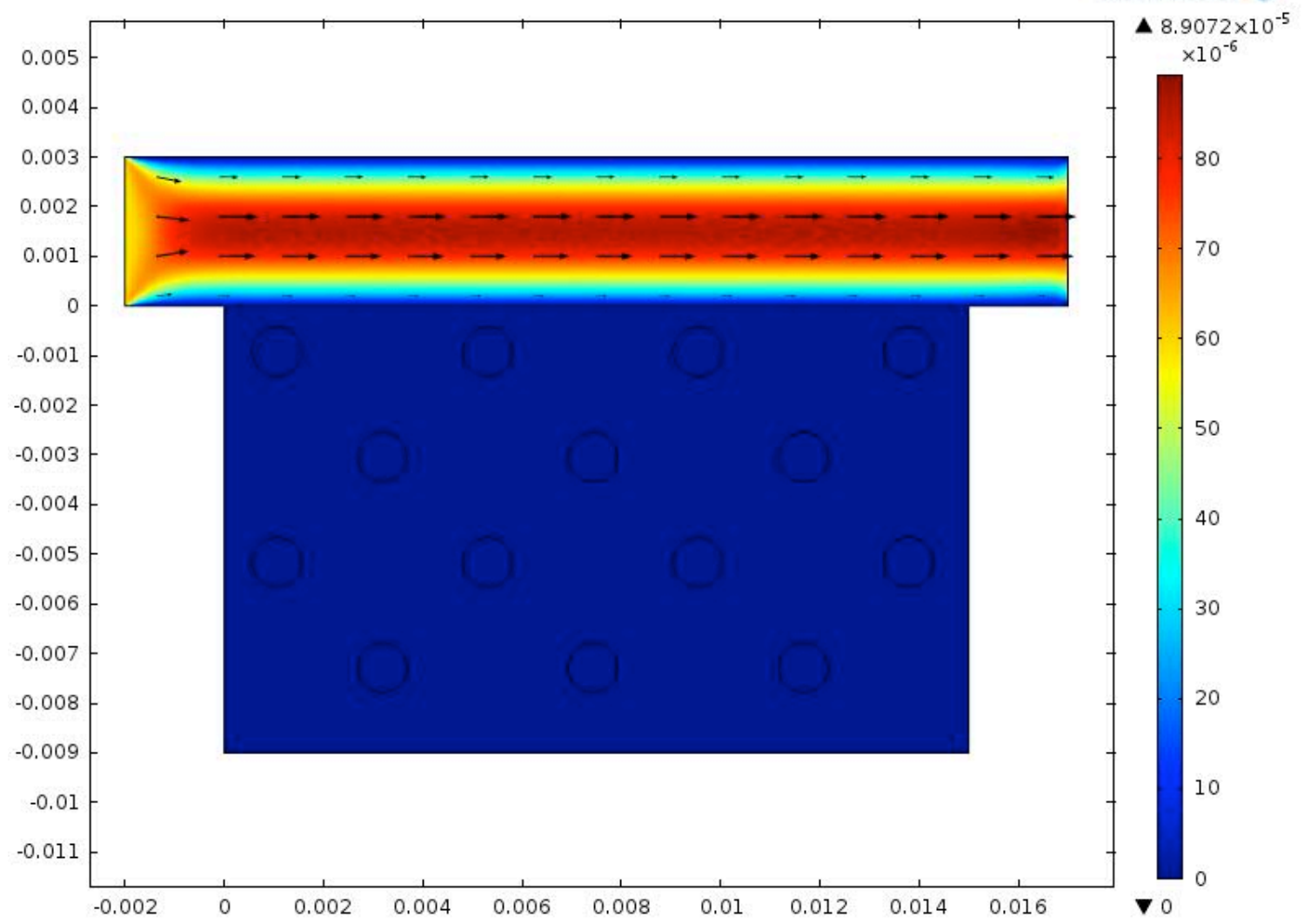

Figure E.10 The simulated flow pattern at $5.0 \mathrm{~m} / \mathrm{d}$ in the H- $\mu$ Chip. Colors toward the red end of the legend bar mean higher values, and colors toward the blue end mean lower values. The black arrows represent the approximate vectors of velocity at certain locations. 